\title{
DESCRIPTION AND ANALYSIS OF THE SECOND SPECTRUM OF VANADIUM (VII)
}

\author{
By William F. Meggers and Charlotte E. Moore ${ }^{1}$
}

\section{ABSTRACT}

Wavelength measurements and intensity estimates are presented for $1,700 \mathrm{~V} \mathrm{II}$ lines with wavelengths ranging from $7015.49 \mathrm{~A}$ in the red to $1313.82 \mathrm{~A}$ in the extreme ultraviolet. The main criterion for selecting these lines was enhancement in the spark as compared with the arc. Supplementary data on furnace-temperature classes and Zeeman patterns for many of these lines support their characterization of singly ionized vanadium atoms.

Analysis of all the available data resulted in the identification of 89 spectral terms, combinations of which account for 1,456, or 86 percent, of the observed lines. The identified terms include 31 singlets, 41 triplets, and 17 quintets. This is the first spectrum in which all theoretical terms arising from electron configurations $d^{3} s$ and $d^{4}$ have been established (except the highest singlet in each set).

The lowest energy state of $\mathrm{V}^{+}$ions is represented by $\left(3 d^{4}\right) a^{5} \mathrm{D}_{0}$, but the strongest emission lines involve $\left(3 d^{3} 4 s\right) a^{5} \mathrm{~F}$, a metastable term. Without doubt, the most intense line of the V II spectrum is $\left(3 d^{3} 4 s\right) a^{5} \mathrm{~F}_{5}-\left(3 d^{3} 4 p\right) z^{5} \mathrm{G}_{6}^{0}$, with wavelength of $3093.108 \mathrm{~A}$, arising from a simple $s-p$ transition.

A great majority of the observed lines are explained as combinations of low (even) terms with middle (odd) terms resulting from the configurations $3 d^{3} 4 p$ and $3 d^{2} 4 s 4 p$. The first high (even) terms probably originate with $3 d^{3} 4 d$ and $3 d^{3} 5 s$, but only fragments of the former and none of the latter could be found.

Line intensities in multiplets, level separations in complex terms, and magnetic splitting factors ( $g$-values) indicate that $L S$ coupling predominates among the four electrons involved in the production of the V II spectrum. With few exceptions, the polyfold terms are regular, and many obey the Landé interval rule rather well. Zeeman-effect observations for 380 lines disclose the $g$-values for 143 atomic-energy levels. A comparison of observed and Landé $g$-sums for levels given by the $d^{3} 4 s$ configuration shows satisfactory compliance with the $g$-sum rule if the scale of the observations is increased 2 to 3 percent.

Guided by theoretical expectations and analogies with similar spectra, the electronic configurations responsible for the observed terms have been identified in nearly all cases.

Inability to establish any levels belonging to the $d^{3} 5 s$ configuration, in series with $d^{3} 4 s$, postpones a spectroscopic determination of the ionization potential of $\mathrm{V}^{+}$ions until this spectrum has been studied under conditions which favor the emission of spectral series. For the present, the only value of this constant is an estimate of 14.1 volts by Russell.

\section{CONTENTS}

I. Introduction

II. Observational data $\ldots \ldots \ldots$

1. Wavelength measurements. 85

2. Intensities _...

3. Temperature classes. 87

4. Zeeman effect_.... 87

5. Absorption and self-reversal

1 Princeton University Observatory. 
III. Term analysis of $\mathrm{V}$ II

1. Lines of the V II spectrum _...

2. Terms of the V II spectrum

3. Electron configurations and spectral terms_.............. 127

IV. References.

\section{INTRODUCTION}

The structure of vanadium spectra was first adopted as a research problem by one of the present authors in 1923, and during that year the first regularities in the arc spectrum of vanadium were announced [1]. ${ }^{2}$ The first regularities among spark lines of vanadium were presented [2] in 1924. These were extended [3] in 1925 and 1926, until approximately 300 lines characteristic of singly ionized vanadium atoms were accounted for as combinations of identified spectral terms.

In those pioneer days of the quantum theory of atomic spectra, regularities in various complex spectra were eargerly sought, primarily for the purpose of testing the proposed alternation and displacement laws of spectral structure [4]. Thus the quartet and sextet multiplets that were found in the $\mathrm{V}$ I spectrum were the first regularities among fifth-group elements, and they verified the predictions of the alternation law. Similarly, discovery in the V II spectrum of triplet and quintet multiplets resembling those of the $\mathrm{Ti}$ i spectrum confirmed the displacement law.

Within a few years the quantum theory of atomic spectra became highly developed (as regards multiplet structure, interpretation of Zeeman patterns [5], correlation of terms with electron configurations [6]), and thus presented analytical spectroscopists with tools and a challenge to classify completely the most complex spectra. But any attempt to interpret a complex spectrum invariably entails much time and labor to observe, compile, and analyze the basic data. Details of exhaustive studies of the first spectrum of vanadium [7] were recently published.

Following 1926, the analysis of the second spectrum of vanadium rested a decade, until it was resumed and advanced to its present state by cooperation of the present authors. From the beginning the term analysis of the vanadium spark spectrum was handicapped by incompleteness and poor quality of wavelength and intensity values, so from time to time efforts were made to improve the fundamental data. Most of the new observing was done at the National Bureau of Standards, and the analysis was extended mainly at Princeton. Preliminary reports of these investigations were presented [8] at scientific meetings in 1938 and 1939 ; and since a stage has now been reached where further observational and analytical activities appear to be unprofitable, we are persuaded to publish our final results.

\section{OBSERVATIONAL DATA}

The analysis of the VII spectrum is based on observed facts consisting of wavelength measurements, intensity estimates, temperature classification, Zeeman effect, absorption and reversal data, all of which are presented in table 1 .

\footnotetext{
${ }_{2}$ Figures in brackets indicate the literature references at the end of this paper.
} 


\section{WAVELENGTH MEASUREMENTS}

Spectroscopic observations of vanadium began in 1866, when Thalén [9] studied the spark spectrum and published the first measurements of the stronger lines. The first attempt to distinguish between arc and spark lines of vanadium was made in 1899 by Lockyer [10], who published a brief list of lines enhanced in the spark as compared with their intensities in the arc. The most extensive table of vanadium spark lines is that of Exner and Haschek [11] published in 1912. It lists 2,837 lines (2131.95 to $6753.3 \mathrm{~A}$, Rowland's scale), but considerably less than half of them appear to be enhanced, and the errors in wavelength values are too large to permit safe use of the combination principle in seeking regularities. Inadequacy of existing data for the purpose of analyzing vanadium spark spectra was recognized in 1924, when a new list of 1,200 enhanced lines (2050 to $6500 \mathrm{~A}$ ) was prepared from observations of juxtaposed arc and spark spectra [3]. These observations were made with vanadium chloride on graphite electrodes, and with spectrographs of moderate dispersion. These results were later displaced by improved ones obtained with pure metal electrodes and more powerful spectrographs. In 1932 Jerome K. Strauss of the Vanadium Corporation of America kindly presented the National Bureau of Standards with massive electrodes of pure vanadium metal for use in spectroscopic investigations. With these electrodes it became possible for the first time to obtain fully exposed spectrograms of vanadium free from impurity lines and undesired lines from graphite, copper, or other electrodes. The spark spectrum of vanadium was accordingly reobserved $(2000$ to $8500 \mathrm{~A})$ with these pure-metal electrodes and the following spectrographs. The ultraviolet range 2000 to 2210 A was recorded on Schumann plates with a Littrow quartz spectrograph giving an average dispersion of about $1 \mathrm{~A} / \mathrm{mm}$ in this range. An ultraviolet interval 2100 to $2800 \mathrm{~A}$ was photographed with a large quartz spectrograph of $3-\mathrm{m}$ focus with $60-\mathrm{cm}$ prism base which produced dispersions of 0.4 to $1.0 \mathrm{~A} / \mathrm{mm}$. The range 2500 to $5000 \mathrm{~A}$ was recorded in the second-order spectrum of a Rowland grating with 20,000 lines per inch, the average scale being $1.7 \mathrm{~A} / \mathrm{mm}$. The longer wave region (5000 to $8500 \mathrm{~A}$ ) was investigated in the first-order spectrum of this grating. The spark for these observations (completed in 1936) was excited by a condensed discharge from an X-ray coil with a high-voltage rating of 50,000. In 1937 an aluminized Pyrex grating with 30,000 lines per inch was obtained from R. W. Wood, and with it the vanadium spark spectrum from 2500 to 8500 A was again observed with 50 percent greater dispersion and complete freedom from ghosts. For this last series of spectrograms the spark was operated by means of a 30,000-volt 60-cycle transformer, but the spectra were indistinguishable from those produced by the X-ray coil.

The schedule of exposures was invariably as follows: (1) Vanaduim conventional arc spectrum, 220 volts, 5 -ampere $d$-c arc, 2 seconds to 1 minute; (2) iron arc spectrum, 1 second to 1 minute; (3) vanadium spark spectrum, primary current 110 volts, 5 to 8 amperes a-c, 0.006 $\mu$ f capacity in parallel with the spark, 2 to 60 minutes. Relative exposure times were chosen so that fully exposed vanadium arc and spark spectrograms resulted in each spectral region with comparable intensities of high-level VI lines. Under such conditions the lines 
enhanced in the spark spectrogram, or appearing only there, positively belong to ionized vanadium atoms. No special effort was made to distinguish between VII and V III lines, but relatively few, if any, of the latter occur above $2600 \mathrm{~A}$. Below $2600 \mathrm{~A}$ the lines appearing only in sparks, and greatly enhanced at the electrodes, are generally found to represent V III and VIV spectra. These have been removed from our V II list.

All wavelength measurements were made relative to international secondary standards of iron in the same spectral order. As usual, the prism spectrograms were reduced with the Hartmann dispersion formula, and the grating spectrograms on the assumption of linear dispersion, small final corrections required by each spectrogram being determined from standards.

A variety of line types occur in the conventional spark spectrum, and obviously not all are measurable with equal precision. Especially difficult are the high-level lines, which in condensed sparks operated at atmospheric pressure always appear hazy and unsymmetrically shaded. Also many V II lines are widened by hyperfine structure which is noticeable with powerful spectrographs.

The visible and infrared spectra of atomic vanadium in atmospheric sources always appear on a background of molecular spectra arising from metallic oxides. Although the molecular spectra are relatively weaker in a disruptive spark than in a continuous arc discharge, they are nevertheless prominent enough in fully exposed spark spectrograms to obscure or falsify faint lines. It is likely that V II lines in general would be sharper, and fainter lines could be observed, in discharges in a partial atmosphere of inert gas; but the present description is confined to the conventional spark in air or in pure nitrogen.

A. G. Shenstone kindly made for the authors a spark spectrogram extending from 2172 to $1308 \mathrm{~A}$ in the extreme ultraviolet. The spark was operated in pure nitrogen, and the spectrum was recorded on a Schumann plate with a normal-incidence vacuum spectrograph having a 2-m glass grating ruled 30,000 lines per inch. The dispersion is $4.2 \mathrm{~A} / \mathrm{mm}$. This spectrogram was measured and reduced relative to $\mathrm{Cu}$ II and Fe III lines identified on the plate. Criteria for the separation of successive spectra of vanadium are lacking, except enhancements at the electrodes, which are assumed to characterize V III and VIV lines. Shorter than $2600 \mathrm{~A}$, only lines believed to belong to the VII spectrum are retained in table 1 , and even these are omitted when unclassified and fainter than 20 below $2000 \mathrm{~A}$, or unclassified below $1550 \mathrm{~A}$.

\section{INTENSITIES}

In this revised description of the V II spectrum an effort was made, for the first time, to assign relative intensities which bear some semblance to reality. The strongest V II line, $3093.11 \mathrm{~A}$, is certainly thousands of times more intense than the faintest observed lines. For such an intensity range the scale of 10 to 1 allows only 10 classes of lines and permits no fine distinctions. Even the scale of 100 to 1 is inadequate, so in the present case we have adopted a scale of 2,500 to 1 . Of course, the intensity values are only visual estimates of line strength based on image width and blackness; but since they are 
usually averages from several fully exposed spectrograms, they are probably comparable over considerable intervals. No quantitative measurements of relative intensities in vanadium spark spectra have ever been made.

When relative intensities are carefully estimated on an open scale, different lines are found to show varying degrees of enhancement from arc to spark exposures. Low-level spark lines usually appear with considerable intensity in ordinary arcs, but high-level spark lines are either weak or nonexistent in light from the central part of an arc. In the spark, low-level lines often show self-reversal, whereas highlevel lines are generally characterized by haziness and dissymmetry. Because degrees of enhancement (as well as other features of the lines) are significant in the analysis of spectral structure, both arc and spark intensities are presented in table 1 .

\section{TEMPERATURE CLASSES}

As stated above, many lines of low or medium intensity in the arc are enhanced in a spark, and such intensity comparisons yield a trustworthy separation of lines due to neutral and to ionized atoms. An independent method of recognizing these enhanced lines is found in a comparison of the arc and high-temperature furnace spectra as studied by King. The enhanced lines are either absent or relatively weak in furnace spectra. In other words the furnace, the arc, and the spark are characterized mainly by differences in ionizing power; and lines belonging to ionized atoms in the arc can be identified either by enhancement in the spark or by diminishment in the furnace. Comparison of the spectra of vanadium excited in a vacuum furnace at various temperatures enabled King [12] to assign, to 1,600 lines (2340 to $6842 \mathrm{~A}$ ) appearing in the arc spectrum, temperature-class numbers that correspond to successive stages of excitation. This classification was an important aid in the analysis of the VI spectrum [7]. Similar data pertinent to the V II spectrum are quoted in table 1 . The assignment of lines to V II, based on arc and furnace comparisons, is confirmed in every case by our independent are and spark comparisons.

\section{ZEEMAN EFFECT}

The Zeeman effect for several hundred vanadium lines (3665 to 6625 A) was published by Babcock [13] in 1911. These observations were improved and extended (to $2314 \mathrm{~A}$ ), and in manuscript form were kindly lent to those actively engaged in multiplet analysis. With the consent of Babcock, the data for V I lines were recently published, together with their interpretation, in a term analysis of the first spectrum of vanadium [7]. The revised data for $\mathrm{V}$ II lines have not been published, but with Babcock's permission they are fully presented in table 1, together with their interpretation. Needless to say, these Zeeman patterns were most helpful in this term analysis of the $\mathrm{V} \mathrm{II}$ spectrum, and they constitute overwhelming confirmation of the quantum numbers assigned to the terms. The observations quoted in table 1 are given in the usual form, that is, the component distances from the undisplaced line are in terms of a normal triplet separation, the $p$-components are in parentheses and are followed by the $n$ components, the strongest components being distinguished by boldface type. The same is true for calculated patterns, except that in 
some cases these are simplified to aid their comparison with the observations.

The calculated patterns are derived from the observed $g$ values (table 5) resulting from Babcock's material quoted in table 1. When the observed components are wholly or partially resolved, the complete computed pattern is given; but when only blends of the components of a given polarization are measured, the calculated position of the blend is given. For the stronger lines this is assumed to coincide with the center of gravity of the theoretical group, as described in the formulas of Shenstone and Blair, and of Russell [14]. These cases are marked $b$ in table 1 . For weak lines it is probable that the measurement applies more nearly to the strongest component of the group, and such cases are marked $s$.

\section{ABSORPTION AND SELF-REVERSAL}

The absorption spectrum of vanadium vapor at $2,000^{\circ} \mathrm{C}$ was investigated by Gieseler and Grotrian [15] to determine the normal state of the atoms. They observed 52 absorption lines (2915 to $6252 \mathrm{~A}$ ), all of which originated either from a low quartet $\mathrm{F}$ or a slightly higher sextet $\mathrm{D}$ term, and concluded that the normal state of neutral vanadium atoms is described by $\left(d^{3} s^{2}\right)^{4} \mathrm{~F}_{11 / 2}$. The only laboratory absorption data extant for V II lines are those by King [16], who observed 10 enhanced lines (2672 to 2691 A) faintly absorbed in vanadium vapor near $2,600^{\circ} \mathrm{C}$. All of these involve the lowest quintet $\mathrm{D}$ term, which is the ground state of the ion. No further laboratory observations of $\mathrm{V}$ II lines in absorption have been made, but additional information about the normal state of $\mathrm{V}^{+}$atoms is found in the phenomenon of self-reversal in the spark. Without exception, the lines which are observed to be more or less self-reversed involve either the low quintet $\mathrm{D}$ term or a slightly higher quintet $\mathrm{F}$ term, and there can be no doubt that the normal state of singly ionized vanadium atoms $\left(\mathrm{V}^{+}\right)$is $\left(d^{4}\right){ }^{5} \mathrm{D}_{0}$.

V II absorption lines are observed in the solar spectrum [17]. The strongest of those observed originate with $\left(d^{3} s\right)^{5} \mathrm{~F}$, because the strongest combinations with $\left(d^{4}\right)^{5} \mathrm{D}$ are shorter than $2900 \mathrm{~A}$ and therefore are not transmitted by the terrestrial atmosphere.

\section{TERM ANALYSIS OF V II}

Since the terms of the V II spectrum are deduced directly from observed properties of the lines, we present in the following order (1) a complete list of observational data, with the addition of term combinations for all classified lines, and calculated Zeeman patterns to compare with observed; (2) facts concerning the established spectral terms; (3) correlation of electron configurations and terms.

\section{LINES OF THE V II SPECTRUM}

Successive columns of table 1 contain the following data: (1) measured wavelength, (2) arc and spark intensity, (3) temperature class, (4) vacuum wave number, (5) term combination, and (6) observed and computed Zeeman patterns.

All wavelengths listed here are on the International Scale; those exceeding $2000 \mathrm{~A}$ are observed in a standard atmosphere, but shorter 
than $2000 \mathrm{~A}$ they are vacuum values. The vacuum wave numbers of the former are derived from Kayser's Table [18].

The letters $a, b, c$, etc., appearing with wavelengths in column 1 have the following meaning:

$a=$ Blend with $\mathrm{V}$ I.

$b=$ Zeeman pattern diffuse.

$c=$ Short-wave member of observed Zeeman pattern double line.

$d=$ Observed Zeeman pattern possible blend with $\mathrm{V}$.

$e=$ Observed Zeeman pattern affected by superposed line.

$f=$ Long-wave member of observed Zeeman pattern double line.

$g=$ Observed Zeeman pattern affected by reversal.

$h=$ Observed Zeeman pattern unsymmetrical; doubtful.

$i=$ Observed Zeeman pattern blended.

$j=$ Intensities in observed Zeeman pattern unsymmetrical.

$k=$ Multiplet designation discordant with observed Zeeman pattern.

$l=$ Short-wave $n$-component of observed Zeeman pattern blended. $m=$ Long-wave $n$-component of observed Zeeman pattern blended.

$n=$ Long-wave $n$-component of observed Zeeman pattern weaker.

$o=n$-component of observed Zeeman pattern blended.

$*=$ Line probably a blend.

Symbols accompanying some of the intensity estimates in column 2 have the following significance, as officially adopted by the International Astronomical Union [20]:

$$
\begin{aligned}
h & =\text { Hazy. } \\
H & =\text { Very hazy. } \\
\mathrm{I} & =\mathrm{V} \text { I. } \\
p & =\text { Part of band structure. } \\
l & =\text { Shaded to longer waves. } \\
s & =\text { Shaded to shorter waves. } \\
d & =\text { Double. } \\
e & =\text { Enhanced at electrode. } \\
r & =\text { Narrow self-reversal. } \\
R & =\text { Wide self-reversal. } \\
B l & =\text { Blend. }
\end{aligned}
$$

In column 3, Roman numerals represent King's temperature class. The accompanying symbols are to be interpreted as follows:

$$
\begin{aligned}
a & =\text { Observed in absorption. } \\
E & =\text { Enhanced line. } \\
r & =\text { Self-reversal. }
\end{aligned}
$$

The term combinations in column 5 show the quantum theoretical interpretation of the observed lines, the notation being that which is in general use [19] and officially adopted by the International Astronomical Union [20].

Symbols accompanying the observed Zeeman patterns in column 6 have the following meanings: $w 1=$ slightly widened; $w 2=$ moderately widened; $w 3=$ greatly widened; while the apparent intensity distribution in such unresolved patterns is qualitatively represented by letters as follows:

For unresolved $p$-components $B=\wedge \mid D=\sqcap ; E=\wedge$.

For unresolved $n$-components $A=\nearrow \backslash ; B=\bigwedge ; C=\sqcap \sqcap$. 
As explained above, the letters $b$ and $s$, appearing with calculated patterns in column 6 , indicate blended- and strongest-component values, respectively. Comparison with values computed from Landé $g$-values may be made by referring to the tables of theoretical Zeeman effects published by Kiess and Meggers [21]. Some remarks on $g$-values and $g$-sums are reserved for presentation in connection with a discussion of V II terms (see next section).

TABLE 1.-Second spectrum of Vanadium

$a=$ absorbed line.

$h=$ Hazy.

$\mathrm{I}=\mathrm{V} \mathrm{I}$.

$p=$ Band line.

$E=$ Enhanced line.

$H=$ Very hazy.

$l=$ Shaded to longer waves.
$R=$ Wide self-reversal.

$r=$ Narrow self-reversal.

$s=$ Shaded to shorter waves

$e=$ Enhanced at electrode.

$d=$ Double.

$\mathrm{II}=\mathrm{V}$ II.

$B l=$ Blend

\begin{tabular}{|c|c|c|c|c|c|c|c|}
\hline 1 & & 2 & 3 & 4 & 5 & \multicolumn{2}{|c|}{6} \\
\hline \multirow{2}{*}{$\lambda_{\mathrm{Bir}} \mathrm{A}$} & \multicolumn{2}{|c|}{ Intensity } & \multirow{2}{*}{$\begin{array}{l}\text { Temp. } \\
\text { elass }\end{array}$} & \multirow{2}{*}{$\begin{array}{l}\text { Wave } \\
\text { No. vac } \\
\mathrm{cm}^{-1}\end{array}$} & \multirow{2}{*}{$\underset{\text { Term }}{\text { combination }}$} & \multicolumn{2}{|c|}{ Zeeman effect } \\
\hline & Arc & Spark & & & & Observed & Computed \\
\hline $\begin{array}{l}7015.49 \\
6987.12 \\
6891.30 \\
6801.16 \\
6748.10\end{array}$ & & $\begin{array}{l}4 h \\
5 h \\
4 h \\
5 \\
2 h\end{array}$ & & $\begin{array}{l}14250.25 \\
14308.11 \\
14507.05 \\
14699.33 \\
14814.90\end{array}$ & $d^{3} \mathrm{P}_{2}-z^{3} \mathrm{P}_{2}^{0}$ & & \\
\hline $\begin{array}{l}6672.84 \\
6517.27 \\
6380.11 \\
6226.29 \\
6120.98\end{array}$ & & $\begin{array}{c}3 h \\
15 h \\
40 h \\
10 h \\
5\end{array}$ & & $\begin{array}{l}14982.00 \\
15339.62 \\
15669.39 \\
16056.50 \\
16332.74\end{array}$ & $\begin{array}{l}b^{1} \mathrm{~F}_{3}-y^{3} \mathrm{~F}_{3}^{\circ} \\
b^{1} \mathrm{~F}_{3}-z^{1} \mathrm{~F}_{3}^{\circ} \\
b^{1} \mathrm{~F}_{3}-z^{1} \mathrm{D}_{2}^{\circ} \\
b^{3} \mathrm{D}_{2}-z^{5} \mathrm{~F}_{2}^{\circ} \\
c^{3} \mathrm{P}_{0}-z^{5} \mathrm{~F}_{1}^{\circ}\end{array}$ & & \\
\hline $\begin{array}{l}6095.93 \\
6086.93 \\
6083.82 \\
6080.11 \\
6031.07\end{array}$ & & $\begin{array}{c}3 \\
15 h \\
10 h \\
6 \\
40\end{array}$ & & $\begin{array}{l}16399.86 \\
16424.11 \\
16432.50 \\
16442.53 \\
16576.23\end{array}$ & $\begin{array}{c}c^{3} \mathrm{P}_{1}-z^{5} \mathrm{~F}_{1}^{\circ} \\
b^{3} \mathrm{D}_{2}-z^{3} \mathrm{D}_{2}^{\circ} \\
b^{3} \mathrm{D}_{1}-z^{3} \mathrm{D}_{1}^{\circ} \\
d^{3} \mathrm{~F}_{4}-z^{3} \mathrm{H}_{4}^{\circ} \\
c^{3} \mathrm{P}_{2}-z^{5} \mathrm{~F}_{3}^{\circ}\end{array}$ & & \\
\hline $\begin{array}{l}6028.98 \\
6028.26 \\
6027.23 \\
6026.81 \\
5967.77\end{array}$ & & $\begin{array}{r}20 \\
40 \\
8 \\
6 \\
6\end{array}$ & & $\begin{array}{l}16581.94 \\
16583.95 \\
16586.79 \\
16587.94 \\
16752.05\end{array}$ & $\begin{array}{c}b^{3} \mathrm{D}_{3}-z^{3} \mathrm{D}_{3}^{\circ} \\
c^{3} \mathrm{P}_{1}-z^{5} \mathrm{~F}_{2}^{\circ} \\
a^{1} \mathrm{~S}_{0}-z^{5} \mathrm{~F}_{1}^{\circ} \\
b^{3} \mathrm{D}_{2}-z^{3} \mathrm{D}_{3}^{\circ} \\
b^{3} \mathrm{D}_{2}-z^{5} \mathrm{D}_{2}^{2}\end{array}$ & & \\
\hline $\begin{array}{l}5951.45 \\
5928.86 \\
5916.364 \\
5914.28 \\
5897.54\end{array}$ & & $\begin{array}{r}4 \\
100 \\
15 \\
5 \\
50\end{array}$ & & $\begin{array}{l}16797.99 \\
16861.99 \\
16897.60 \\
16903.56 \\
16951.54\end{array}$ & $\begin{array}{c}c^{3} \mathrm{P}_{0}-z^{3} \mathrm{D}_{1}^{\circ} \\
c^{3} \mathrm{P}_{2}-z^{3} \mathrm{D}_{3}^{\circ} \\
b^{3} \mathrm{D}_{3}-z^{5} \mathrm{D}_{3}^{\circ} \\
b^{3} \mathrm{D}_{2}-z^{5} \mathrm{D}_{3}^{\circ} \\
c^{3} \mathrm{P}_{1}-z^{3} \mathrm{D}_{2}^{\circ}\end{array}$ & $\begin{array}{l}\text { (0) } 1.31 \\
\text { (0) } 0.96\end{array}$ & $\begin{array}{l}\text { (0) } 1.28 b \\
\text { (0) } 0.94 b\end{array}$ \\
\hline $\begin{array}{l}5862.80 \\
5819.93 \\
5791.47 \\
5642.01 \\
5562.02\end{array}$ & $p$ & $\begin{array}{l}15+p ? \\
80 \\
15 \\
60 h \\
\quad 4 h p ?\end{array}$ & & $\begin{array}{l}17051.98 \\
17177.59 \\
17262.00 \\
17719.27 \\
17974.10\end{array}$ & $\begin{array}{l}a^{1} \mathrm{~S}_{0}-z^{3} \mathrm{D}_{1}^{\circ} \\
c^{3} \mathrm{P}_{2}-z^{3} \mathrm{D}_{3}^{3} \\
c^{1} \mathrm{G}_{4}-y^{1} \mathrm{G}_{4}^{\circ} \\
c^{3} \mathrm{D}_{2}-w^{3} \mathrm{~F}_{3}^{3}\end{array}$ & $\begin{array}{l}\text { (0) } 1.50 \\
\text { (0) } 0.97\end{array}$ & $\begin{array}{l}(0) 1.58 b \\
(0.13) 0.98 b\end{array}$ \\
\hline $\begin{array}{l}5530.10 \\
5487.00 \\
5457.10 \\
5439.30 \\
5432.09\end{array}$ & & $\begin{array}{r}4 \\
8 \\
4 \\
15 \\
15\end{array}$ & & $\begin{array}{l}18077.85 \\
18219.85 \\
18319.67 \\
18379.62 \\
18404.02\end{array}$ & $\begin{array}{l}c^{3} \mathrm{D}_{3}-w^{3} \mathrm{~F}_{4}^{\circ} \\
a^{3} \mathrm{D}_{1}-z^{5} \mathrm{~F}_{1}^{\circ} \\
a^{3} \mathrm{D}_{3}-z^{5} \mathrm{~F}_{2}^{\circ} \\
a^{3} \mathrm{D}_{2}-z^{5} \mathrm{~F}_{2}^{\circ} \\
a^{3} \mathrm{D}_{1}-z^{5} \mathrm{~F}_{2}^{\circ}\end{array}$ & & \\
\hline $\begin{array}{l}5384.89 \\
5357.35 \\
5350.37 \\
5349.75 \\
5341.22\end{array}$ & & $\begin{array}{l}8 \\
2 \\
5 \\
3 \\
2\end{array}$ & & $\begin{array}{l}18565.33 \\
18660.77 \\
18685.11 \\
18687.28 \\
18717.12\end{array}$ & $\begin{array}{l}a^{3} \mathrm{D}_{3}-z^{3} \mathrm{~F}_{3}^{0} \\
a^{3} \mathrm{D}_{2}-z^{3} \mathrm{D}_{1}^{\circ} \\
a^{3} \mathrm{D}_{1}-z^{3} \mathrm{D}_{1}^{\circ} \\
a^{3} \mathrm{D}_{3}-z^{3} \mathrm{D}_{2}^{\circ} \\
c^{1} \mathrm{G}_{4}-y^{1} \mathrm{~F}_{3}^{\circ}\end{array}$ & $\begin{array}{l}(0 ?) 1.29 p w_{1} ; n w_{1} \mathrm{~B} ? \\
(0.30) 0.37\end{array}$ & $\begin{array}{l}(0.15) 1.27 b \\
(0.25) 0.36 b\end{array}$ \\
\hline $\begin{array}{l}5332.65 \\
5322.81 \\
5303.26 \\
5290.74 \\
5288.31\end{array}$ & $p$ & $\begin{array}{l}9 \\
5 \\
40 \\
6+I \\
5\end{array}$ & & $\begin{array}{l}18747.20 \\
18781.86 \\
18851.09 \\
18895.70 \\
18904.39\end{array}$ & $\begin{array}{r}a^{3} \mathrm{D}_{2}-z^{3} \mathrm{D}_{2}^{\circ} \\
c^{1} \mathrm{G}_{4}-x^{3} \mathrm{G}_{5}^{\circ} \\
a^{3} \mathrm{D}_{3}-z^{3} \mathrm{D}_{3}^{\circ} \\
d^{3} \mathrm{~F}_{2}-z^{1} \mathrm{~F}_{3}^{\circ} \\
c^{3} \mathrm{~F}_{3}-y^{3} \mathrm{~F}_{3}^{\circ}\end{array}$ & $\begin{array}{l}\text { (0) } 1.15 \\
\text { (?) } 1.49 \mathrm{pw}_{2} \mathrm{D} ; n w_{2} \mathrm{C} \\
\text { (0) } 1.37 \\
\text { (0) } 1.20\end{array}$ & $\begin{array}{l}(0.09) 1.10 b \\
(0) 1.53 b \\
(0.05) 1.31 b \\
(0) 1.27 b\end{array}$ \\
\hline
\end{tabular}

See footnotes at end of table. 
TABLE 1.-Second spectrum of Vanadium-Continued

\begin{tabular}{|c|c|c|c|c|c|c|c|}
\hline \multirow{3}{*}{$\lambda_{\mathrm{a} \text { ir } \mathrm{A}}$} & \multicolumn{2}{|c|}{2} & 3 & 4 & 5 & 6 & \\
\hline & & nsity & \multirow{2}{*}{$\begin{array}{l}\text { Temp. } \\
\text { class }\end{array}$} & \multirow{2}{*}{$\begin{array}{l}\text { Wave } \\
\text { No. vac }\end{array}$} & \multirow{2}{*}{$\begin{array}{c}\text { Term } \\
\text { combination }\end{array}$} & \multicolumn{2}{|c|}{ Zeeman effect } \\
\hline & Arc & Spark & & & & Observed & Computed \\
\hline $\begin{array}{l}5280.00 \\
5275.65^{\mathrm{a}} \\
5263.99 \\
5249.22 \\
5241.19\end{array}$ & $?$ & $\begin{array}{c}3 ? \\
10+\mathrm{I} \\
15 \\
1 ? \\
100 h\end{array}$ & & $\begin{array}{l}\text { 18934. } 14 \\
18949.75 \\
18991.72 \\
19045.16 \\
19074.34\end{array}$ & $\begin{array}{l}c^{3} \mathrm{~F}_{2}-y^{3} \mathrm{~F}_{2}^{\circ} \\
c^{3} \mathrm{~F}_{4}-y^{3} \mathrm{~F}_{4}^{\circ} \\
b^{3} \mathrm{H}_{4}-z^{3} \mathrm{G}_{3}^{0} \\
d^{3} \mathrm{P}_{2}-y^{3} \mathrm{D}_{3}^{\circ} \\
c^{1} \mathrm{G}_{4}-y^{1} \mathrm{H}_{5}^{\circ}\end{array}$ & $\begin{array}{l}(0.61) 1.27 \\
(0) 0.88 \\
\text { (0) } 1.16\end{array}$ & $\begin{array}{l}\text { (0.23) } 1.22 \\
\text { (0) } 0.79 b \\
\text { (0) } 1.17 b\end{array}$ \\
\hline $\begin{array}{l}5232.82 \\
5227.70 \\
5215.928 \\
5214.98 \\
5202.94\end{array}$ & $p$ & $\begin{array}{r}2 \\
20 \\
25 \\
2 \\
2\end{array}$ & & $\begin{array}{l}\text { 19104. } 85 \\
19123.56 \\
19166.72 \\
19170.21 \\
19214.57\end{array}$ & $\begin{array}{l}b^{3} \mathrm{H}_{5}-z{ }^{3} \mathrm{G}_{4}^{\circ} \\
a^{3} \mathrm{D}_{3}-z{ }^{5} \mathrm{D}_{3}^{\circ} \\
a^{1} \mathrm{D}_{2}-z^{3} \mathrm{~F}_{3}^{\circ}\end{array}$ & (?) $1.42 p w_{2} \mathrm{D}$ & (0.44) $1.38 b$ \\
\hline $\begin{array}{l}5199.68 \\
5191.59 \\
5157.28 \\
5151.87 \\
5143.89\end{array}$ & $p$ & $\begin{array}{l}4 \\
2 \\
2 ? \\
2 \\
3\end{array}$ & & $\begin{array}{l}\text { 19226. } 61 \\
\text { 19256. } 58 \\
\text { 19384. } 69 \\
\text { 19405. } 03 \\
19435.14\end{array}$ & $\begin{array}{c}a^{3} \mathrm{D}_{2}-z{ }^{5} \mathrm{D}_{3}^{2} \\
d^{3} \mathrm{~F}_{3}-z^{1} \mathrm{D}_{2}^{2} \\
b^{3} \mathrm{D}_{2}-z{ }^{3} \mathrm{~F}_{2}^{0} \\
c^{3} \mathrm{~F}_{4}-z^{1} \mathrm{G}_{4}^{\circ}\end{array}$ & & \\
\hline $\begin{array}{l}5132.19 \\
5106.233 \\
5060.67\end{array}$ & $?$ & $\begin{array}{r}2 \\
5 \\
15\end{array}$ & & $\begin{array}{l}19479.45 \\
19578.47 \\
19754.74\end{array}$ & $\begin{array}{l}b{ }^{3} \mathrm{D}_{1}-z^{3} \mathrm{~F}_{2}^{2} \\
b^{3} \mathrm{D}_{2}-z^{3} \mathrm{~F}_{3}^{3}\end{array}$ & $\begin{array}{l}\text { (0) } 0.83 \\
(0 ?) 1.16 p w_{2} \mathrm{D} ; n w_{2}\end{array}$ & (0) $0.79 b$ \\
\hline $\begin{array}{l}5048.91 \\
5047.308\end{array}$ & & $\begin{array}{l}15 \\
10+\mathrm{I}\end{array}$ & & $\begin{array}{l}19800.75 \\
19807.04\end{array}$ & $\begin{array}{l}d^{3} \mathrm{~F}_{2}-y^{3} \mathrm{D}_{1}^{\circ} \\
b^{3} \mathrm{D}_{3}-z^{3} \mathrm{~F}_{4}^{\circ}\end{array}$ & $\begin{array}{l}\text { (0) } 0.73 \\
\text { (0) } 1.05\end{array}$ & $\begin{array}{l}\text { (0) } 0.76 b \\
\text { (0) } 1.10 \mathrm{~s}\end{array}$ \\
\hline $\begin{array}{l}5019.855 \\
5016.60 \\
4973.16 \\
4968.50 \\
4965.40\end{array}$ & & $\begin{array}{c}100 h \\
4 \\
2 \\
1 \\
40\end{array}$ & & $\begin{array}{l}\text { 19915. } 36 \\
19928.28 \\
20102.34 \\
20121.20 \\
20133.76\end{array}$ & $\begin{array}{l}b^{1}{ }^{1} \mathrm{~F}_{3}-y \\
c^{1}{ }^{1} \mathrm{D}_{4} \\
d^{2}-x \\
d^{3} \mathrm{~F}_{2}-y^{1} \mathrm{D}_{2}^{3} \\
b^{1} \mathrm{D}_{4}-2 \\
d^{3} \mathrm{G}_{3}^{3} \mathrm{G}_{3}-y^{3}{ }^{3} \mathrm{D}_{2}^{\circ}\end{array}$ & (0) 1.03 & $\begin{array}{l}\text { (0) } 1.00 b \\
\text { (0) } 0.99 b\end{array}$ \\
\hline 4963.75 & & 2 & & 20140.46 & $d^{3} \mathrm{P}_{2}-z^{3} \mathrm{~S}_{1}$ & (?) $1.16 p w_{1} \mathrm{D} ; n w_{1}$ & (0) $1.14 b$ \\
\hline $\begin{array}{l}4947.58 \\
4912.38\end{array}$ & & $\begin{array}{r}40 \\
2\end{array}$ & & $\begin{array}{l}20206.28 \\
20351.07\end{array}$ & $\begin{array}{l}c^{3} \mathrm{~F}_{2}-y^{3} \mathrm{D}^{\circ} \mathrm{i} \\
d^{3} \mathrm{P}_{2}-x^{3} \mathrm{~F}_{3}^{\circ}\end{array}$ & (0?) $0.75 \mathrm{pw}_{2} \mathrm{D}$ & (0) $0.76 b$ \\
\hline $\begin{array}{l}4884.06 \\
4883.415\end{array}$ & & $\begin{array}{r}50 \\
100\end{array}$ & & $\begin{array}{l}20469.07 \\
20471.77\end{array}$ & $\begin{array}{l}c^{3} \mathrm{~F}_{3}-y^{3} \mathrm{D}_{2}^{\circ} \\
\mathrm{d}^{3} \mathrm{~F}_{4}-y^{3} \mathrm{D}_{3}^{\circ}\end{array}$ & $\begin{array}{l}\text { (0?) } 1.02 p w_{1} \mathrm{D} \\
\text { (?) } 1.14 p w_{2} \mathrm{D}\end{array}$ & $\begin{array}{l}\text { (0) } 1.01 b \\
\text { (0) } 1.17 b\end{array}$ \\
\hline $\begin{array}{l}4874.805 \\
4842.50 \\
4839.08\end{array}$ & & $\begin{array}{l}4 \\
2 h \\
3\end{array}$ & & $\begin{array}{l}20507.93 \\
20644.74 \\
20659.33\end{array}$ & $\begin{array}{l}c^{3} \mathrm{~F}_{2}-y^{3} \mathrm{D}_{2}^{2} \\
c^{3} \mathrm{D}_{2}-v^{3} \mathrm{D}_{2}^{0} \\
d^{3} \mathrm{P}_{2}-x^{3} \mathrm{D}_{2}^{8}\end{array}$ & $\begin{array}{l}\text { (?) } 1.15 p w_{1} \mathrm{D} ; n w_{1} \\
\mathrm{~B} \text { ? }\end{array}$ & $(0.50) 1.24 b$ \\
\hline $\begin{array}{l}4823.396 \\
4813.952^{b}\end{array}$ & $3 ?$ & $\begin{array}{r}6 \\
50\end{array}$ & & $\begin{array}{l}20726.50 \\
20767.17\end{array}$ & $\begin{array}{l}d^{3}{ }^{3} \mathrm{P}_{2}-x^{3} \mathrm{D}_{3}^{3} \\
c^{3} \mathrm{~F}_{4}-y^{3} \mathrm{D}_{3}^{3}\end{array}$ & $\begin{array}{l}\text { (?) } 1.15 p w_{1} \mathrm{D} \\
\text { (0) } 1.15\end{array}$ & $\begin{array}{l}\text { (0) } 1.14 b \\
\text { (0) } 1.19 s\end{array}$ \\
\hline $\begin{array}{l}4813.00 \\
4811.14\end{array}$ & & $\begin{array}{l}1 ? \\
6\end{array}$ & & $\begin{array}{l}\text { 20771. } 27 \\
20779.30\end{array}$ & $\begin{array}{l}c^{3} \mathrm{D}_{2}-v^{3} \mathrm{D}_{1}^{\circ} \\
c^{3} \mathrm{~F}_{3}-y^{3} \mathrm{D}_{3}^{3}\end{array}$ & $(0.64) 1.24 n w_{2}$ & $\begin{array}{r}(0.21,0.42,0.63) \\
0.64,0.85, \mathbf{1 . 0 6}, \\
\mathbf{1 . 2 7}, 1.48,1.69\end{array}$ \\
\hline $\begin{array}{l}4810.17 \\
4737.59 \\
4634.21\end{array}$ & & $\begin{array}{l}1 ? \\
1 \\
3 h\end{array}$ & & $\begin{array}{l}20783.49 \\
21101.89 \\
21572.63\end{array}$ & $\begin{array}{l}b^{3} \mathrm{G}_{4}-z^{3} \mathrm{D}_{3}^{8} \\
b^{3} \mathrm{~F}_{2}-z^{3} \mathrm{G}_{2}^{\circ} \\
d^{3} \mathrm{~F}_{2}-x^{3} \mathrm{~F}_{2}^{\circ}\end{array}$ & & \\
\hline $\begin{array}{l}4627.48 \\
4618.52 \\
4605.352 \\
4600.19 \\
4596.37\end{array}$ & & $\begin{array}{r}1 \\
3 \\
15 \\
150 \\
5 h\end{array}$ & & $\begin{array}{l}21604.00 \\
21645.91 \\
21707.80 \\
21732.16 \\
21750.22\end{array}$ & $\begin{array}{l}d^{3} \mathrm{~F}_{3}-x^{3} \mathrm{~F}_{2}^{0} \\
c^{1} \mathrm{D}_{2}-x^{1} \mathrm{~F}_{3}^{\circ} \\
a^{3} \mathrm{D}_{2}-z^{3} \mathrm{~F}_{2}^{\circ} \\
a^{3} \mathrm{D}_{1}-z^{3} \mathrm{~F}_{3}^{\circ} \\
d^{3} \mathrm{~F}_{3}-x^{3} \mathrm{~F}_{3}^{\circ}\end{array}$ & (0)0.77 & (0) $0.73 b$ \\
\hline $\begin{array}{l}4590.505 \\
4564.592 \\
4558.46 \\
4556.765 \\
4538.64\end{array}$ & 4 & $\begin{array}{c}7 h \\
200 \\
20 \\
4 \\
2\end{array}$ & & $\begin{array}{l}21778.01 \\
21901.64 \\
21931.10 \\
21939.26 \\
22026.87\end{array}$ & $\begin{array}{l}d^{3} \mathrm{~F}_{4}-x^{3} \mathrm{~F}_{3}^{\circ} \\
a^{3} \mathrm{D}_{2}-z^{3} \mathrm{~F}_{3}^{0} \\
d^{3} \mathrm{~F}_{2}-x^{3} \mathrm{D}_{1}^{\circ} \\
c^{3} \mathrm{~F}_{3}-x^{3} \mathrm{~F}_{2}^{\circ} \\
d^{3} \mathrm{~F}_{2}-x^{3} \mathrm{D}_{2}^{\circ}\end{array}$ & $\begin{array}{l}\text { (?) } 1.44 \\
(0) 0.97 p w_{1} \mathrm{D} ; n w_{1} \mathrm{~A}\end{array}$ & $\begin{array}{l}\text { (0) } 1.47 b \\
\text { (0) } 0.91 b\end{array}$ \\
\hline $\begin{array}{l}4535.215 \\
4532.188 \\
4529.08 \\
4528.51 \\
4520.55\end{array}$ & 3 & $\begin{array}{c}3 h \\
40 h \\
5 ? \\
300 \\
5\end{array}$ & V $E$ & $\begin{array}{l}22043.51 \\
22058.23 \\
22073.37 \\
22076.15 \\
22115.02\end{array}$ & $\begin{array}{l}d^{3} \mathrm{~F}_{4}-x^{3} \mathrm{~F}_{4}^{\circ} \\
d^{3} \mathrm{~F}_{3}-x^{3} \mathrm{D}_{2}^{2} \\
c^{3} \mathrm{~F}_{4}-x^{3} \mathrm{~F}_{3}^{0} \\
a^{3} \mathrm{D}_{3}-z^{3} \mathrm{~F}_{4}^{0}\end{array}$ & $\begin{array}{l}(0) 1.02 \\
(0 ?) 1.13\end{array}$ & $\begin{array}{l}(0) 1.00 b \\
(0) 1.10 b\end{array}$ \\
\hline $\begin{array}{l}4518.38 \\
4517.35 \\
4512.72 \\
4483.50 \\
4475.70\end{array}$ & & $\begin{array}{r}2 h \\
3 h \\
60 h \\
2 h \\
20 h\end{array}$ & & $\begin{array}{l}22125.64 \\
22130.69 \\
22153.39 \\
22297.77 \\
22336.63\end{array}$ & $\begin{array}{l}d^{3} \mathrm{~F}_{3}-x^{3} \mathrm{D}_{3}^{3} \\
d^{3} \mathrm{~F}_{2}-2{ }^{1} \mathrm{P}_{1}^{0} \\
d^{3} \mathrm{~F}_{4}-x x^{3} \mathrm{D}_{3}^{\circ} \\
d^{3} \mathrm{P}_{0}-x x^{3} \mathrm{P}_{1}^{0} \\
c^{3} \mathrm{~F}_{2}-x^{3} \mathrm{D}_{1}^{\circ}\end{array}$ & $\begin{array}{l}(0) 1.20 \\
(0) 0.66\end{array}$ & $\begin{array}{l}(0) 1.18 b \\
(0) 0.69 b\end{array}$ \\
\hline
\end{tabular}

See footnotes at end of table. 
TABLE 1.-Second spectrum of Vanadium-Continued

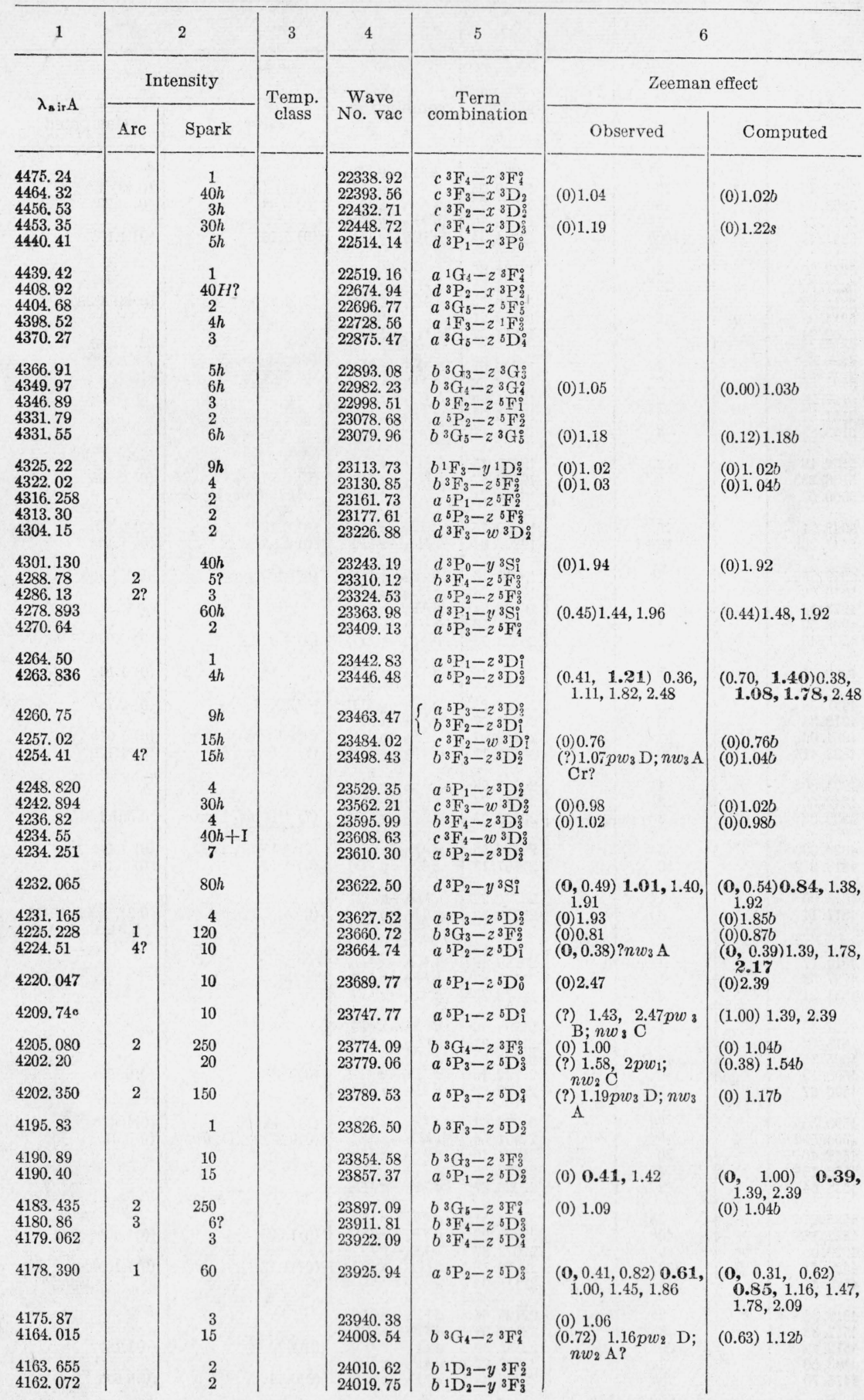

See footnotes at end of table. 
TABLE 1.-Second spectrum of Vanadium-Continued

\begin{tabular}{|c|c|c|c|c|c|c|c|}
\hline \multirow{3}{*}{$\lambda_{\mathrm{a} \text { ir } \mathrm{A}}$} & \multicolumn{2}{|c|}{2} & 3 & 4 & 5 & \multicolumn{2}{|c|}{6} \\
\hline & \multicolumn{2}{|c|}{ Intensity } & \multirow{2}{*}{$\begin{array}{l}\text { Temp. } \\
\text { class }\end{array}$} & \multirow{2}{*}{$\begin{array}{l}\text { Wave } \\
\text { No. vac }\end{array}$} & \multirow{2}{*}{$\begin{array}{c}\text { Term } \\
\text { combination }\end{array}$} & \multicolumn{2}{|c|}{ Zeeman effect } \\
\hline & Arc & Spark & & & & Observed & Computed \\
\hline $\begin{array}{l}4142.90 \\
4140.08\end{array}$ & $5 ?$ & $\frac{6+\mathrm{I}}{2}$ & & $\begin{array}{l}24130.90 \\
24147.34\end{array}$ & $d^{3} \mathrm{P}_{2}-y{ }^{1} \mathrm{P}_{1}^{\circ}$ & $\stackrel{(0 ?)}{\mathrm{B}} 0.59 p w_{2} \mathrm{D} ; n w_{2}$ & \\
\hline $\begin{array}{l}4101.00 \\
4085.67 \mathrm{~b} \\
4080.44\end{array}$ & $4 ?$ & $\begin{array}{c}8+\mathrm{I} \\
10 h \\
2\end{array}$ & & $\begin{array}{l}24377.44 \\
24468.91 \\
24500.27\end{array}$ & $\begin{array}{l}b^{1} \mathrm{D}_{2}-z^{1} \mathrm{~T}_{3}^{\circ} \\
d^{3} \mathrm{~F}_{2}-y^{1} \mathrm{~F}_{3}^{2} \\
d^{3} \mathrm{~F}_{3}-y^{1} \mathrm{~F}_{3}^{0}\end{array}$ & $\begin{array}{l}\text { (0) } 0.94 \\
\text { (?) } 1.24 p w_{2} \mathrm{D} ; n w_{2}\end{array}$ & $\begin{array}{l}\text { (0) } 0.95 b \\
\text { (0) } 1.21 b\end{array}$ \\
\hline $\begin{array}{l}4075.66 \\
4067.03\end{array}$ & & $\begin{array}{l}3 \\
5\end{array}$ & & $\begin{array}{l}24529.00 \\
24581.05\end{array}$ & $\begin{array}{l}a^{3} \mathrm{H}_{5}-z^{5} \mathrm{~F}_{4}^{\circ} \\
a^{3} \mathrm{P}_{2}-z^{5} \mathrm{~F}_{1}^{0}\end{array}$ & $\left.\begin{array}{l}(\mathbf{0}, 1.16) \\
\mathbf{2 . 6 2}\end{array}\right) .39,1.50$ & $\begin{array}{l}(0,1.14) \\
2.63\end{array}$ \\
\hline $\begin{array}{l}4065.070^{\mathrm{b}} \\
4056.270 \\
4053.59\end{array}$ & & $\begin{array}{c}100 \\
7 \\
60 l\end{array}$ & & $\begin{array}{l}24592.90 \\
24646.26 \\
24662.55\end{array}$ & $\begin{array}{l}d^{3} \mathrm{~F}_{4}-x^{3} \mathrm{G}_{5}^{\circ} \\
a^{3} \mathrm{H}_{6}-z^{5} \mathrm{~F}_{5}^{5} \\
d^{3} \mathrm{~F}_{3}-x^{3} \mathrm{G}_{4}^{8}\end{array}$ & $\begin{array}{l}\text { (0) } 0.92 \\
\text { (?) } 0.98 p w_{3} \mathrm{D} \\
\text { (0) } 0.97\end{array}$ & $\begin{array}{l}\text { (0) } 0.99 b \\
\text { (0) } 0.94 b \\
\text { (0) } 0.98 b\end{array}$ \\
\hline $\begin{array}{l}4051.34 \\
4051.06 \mathrm{~d} \\
4049.03 \\
4046.269 \\
4039.574\end{array}$ & 50 & $\begin{array}{l}100+\mathrm{I} \\
20+\mathrm{I} \\
3 \\
50 \\
20\end{array}$ & & $\begin{array}{l}24676.24 \\
24677.95 \\
24690.32 \\
24707.17 \\
24748.12\end{array}$ & $\begin{array}{l}d^{3} \mathrm{~F}_{2}-x^{3} \mathrm{G}_{3}^{\circ} \\
a^{3} \mathrm{G}_{4}-z^{3} \mathrm{G}_{3}^{3} \\
d^{3} \mathrm{~F}_{4}-x^{3} \mathrm{G}_{4}^{8} \\
b^{1} \mathrm{D}^{2}-z^{1} \mathrm{D}_{2}^{2} \\
a^{3} \mathrm{G}_{5}-z^{3} \mathrm{G}_{4}^{8}\end{array}$ & $\begin{array}{l}\text { (0) } 1.15 \\
(0) 0.95 \\
(0 ?) \quad 1.78 p w_{3} \quad \mathrm{D} \text {; } \\
n w_{2} \mathrm{~A}\end{array}$ & $\begin{array}{l}\text { (0) } 1.24 b \\
(0.11) 0.96 b \\
\text { (0) } 1.73 \mathrm{~s}\end{array}$ \\
\hline $\begin{array}{l}4038.545 \\
4036.779\end{array}$ & 3 & $\begin{array}{r}2 \\
60\end{array}$ & V $E$ & $\begin{array}{l}24754.43 \\
24765.25\end{array}$ & $\begin{array}{l}a^{1} \mathrm{P}_{1}-y{ }^{5} \mathrm{D}_{0}^{0} \\
a^{3} \mathrm{P}_{2}-z^{5} \mathrm{~F}_{2}^{\circ}\end{array}$ & $\begin{array}{l}\text { (0) } 0.99 \\
(0.38,0.80) 0.66, \\
1.08,1.49,1.88\end{array}$ & $\begin{array}{l}\text { (0) } 0.97 \\
(0.41,0.82) 0.67 \text {, } \\
1.08,1.49,1.90\end{array}$ \\
\hline $\begin{array}{l}4035.631 \\
4027.30\end{array}$ & 10 & $\begin{array}{r}400 \\
1\end{array}$ & V $E$ & $\begin{array}{l}24772.30 \\
24823.54\end{array}$ & $\begin{array}{l}a^{3} \mathrm{G}_{3}-z^{3} \mathrm{G}_{3}^{\circ} \\
c^{3} \mathrm{~F}_{4}-y^{1} \mathrm{~F}_{3}^{\circ}\end{array}$ & (0) 0.75 & $(0.26) 0.79 b$ \\
\hline 4023.388 & 12 & 600 & V $E$ & 24847.68 & $a^{3} \mathrm{G}_{1}-z^{3} \mathrm{G}_{4}$ & $\left\{\begin{array}{ll}(0) & 0.85 \\
(0) & 1.02\end{array}\right\}$ & $(0.10) 1.02 b$ \\
\hline $\begin{array}{l}4019.05 \\
4017.29\end{array}$ & & $\begin{array}{r}7 h \\
15 h\end{array}$ & & $\begin{array}{l}24874.50 \\
24885.39\end{array}$ & $\begin{array}{l}c^{3} \mathrm{~F}_{2}-y^{1} \mathrm{~F}_{3}^{\circ} \\
d^{3} \mathrm{~F}_{1}-y^{1} \mathrm{H}_{5}^{\circ}\end{array}$ & & \\
\hline 4016.82 & & $20 h$ & & 24888.31 & $c^{3} \mathrm{~F}_{4}-\mathrm{x}^{3} \mathrm{G}_{5}^{0}$ & 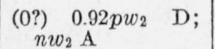 & (0) $0.95 b$ \\
\hline 4008.17 & 1 & 20 & & 24942.02 & $a^{3} \mathrm{G}_{3}-z^{3} \mathrm{G}_{4}^{\circ}$ & (?) ${ }_{n w_{3}, \mathrm{~B}} 1.91 w_{3} \mathrm{D}$; & (0) $1.90 \mathrm{~s}$ \\
\hline 4005.712 & 15 & 800 & $\mathrm{~V}$ & 24957.32 & $a^{3} \mathrm{G}_{5}-z^{3} \mathrm{G}_{5}^{0}$ & (0) 1.17 & $(0.08) 1.18 b$ \\
\hline $\begin{array}{l}4002.940 \\
4001.17\end{array}$ & 4 & 80 & V $E$ & $\begin{array}{l}24974.60 \\
24985.65\end{array}$ & $\begin{array}{l}a^{3} \mathrm{P}_{1}-z^{5} \mathrm{~F}_{1}^{\circ} \\
c^{3} \mathrm{~F}_{4}-x^{3} \mathrm{G}_{4}^{8}\end{array}$ & (1.18) $0.29,1.47$ & (1.13) $0.35,1.48$ \\
\hline $\begin{array}{l}3999.195 \\
3997.126\end{array}$ & 5 & $\begin{array}{l}30 h \\
200\end{array}$ & V $E$ & $\begin{array}{l}24997.99 \\
25010.93\end{array}$ & $\begin{array}{l}c^{3} \mathrm{~F}_{3}-x^{3} \mathrm{G}_{4}^{0} \\
a^{3} \mathrm{P}_{2}-z^{5} \mathrm{~F}_{3}^{0}\end{array}$ & $\begin{array}{l}\text { (0) } 0.95 \\
\text { (?) } 1.00 w_{2} \quad \mathrm{D} \text {; } \\
n w_{2} \mathrm{~A}\end{array}$ & $\begin{array}{l}(0) 0.96 b \\
\text { (0) } 0.99 b\end{array}$ \\
\hline 3991.965 & & 2 & & 25043.26 & $\left\{\begin{array}{l}c^{3} \mathrm{~F}_{3}-x^{3} \mathrm{G}_{3}^{\circ} \\
d^{3} \mathrm{P}_{1}-y^{1} \mathrm{D}_{2}^{\circ}\end{array}\right.$ & & \\
\hline $\begin{array}{l}3991.47 \\
3989.803 \\
3985.783\end{array}$ & 1 & $\begin{array}{r}3 \\
15 \\
30\end{array}$ & & $\begin{array}{l}25046.37 \\
25056.83 \\
25082.10\end{array}$ & $\begin{array}{l}a^{3} \mathrm{P}_{2}-z^{3} \mathrm{D}_{1}^{\circ} \\
a^{3} \mathrm{G}_{4}-z^{3} \mathrm{G}_{5}^{\circ} \\
c^{3} \mathrm{~F}_{2}-x^{3} \mathrm{G}_{3}^{0}\end{array}$ & $\begin{array}{ll}\text { (0) } 1.64 & \\
(?) 1.02 p w_{2} & \mathrm{D} \text {; }\end{array}$ & $\begin{array}{l}\text { (0) } 1.57 b \\
\text { (0) } 0.97 b\end{array}$ \\
\hline 3977.732 & 2 & 60 & & 25132.87 & $a^{3} \mathrm{P}_{2}-z^{3} \mathrm{D}_{2}^{\circ}$ & $\begin{array}{r}n w_{2} B \\
(0.36,0.77) 0.68, \\
\mathbf{1 . 1 0}, \mathbf{1 . 4 9}, 1.87\end{array}$ & $\begin{array}{r}(0.41,0.82) \\
\quad 1.08,1.49,1.90\end{array}$ \\
\hline 3973.642 & 8 & 300 & & 25158.74 & $a^{3} \mathrm{P}_{1}-z^{5} \mathrm{~F}_{2}^{\circ}$ & $\begin{array}{l}(\mathbf{0}, 0.47) \quad \mathbf{0 . 6 2} \\
1.10,1.51\end{array}$ & $\begin{array}{c}(0,0.40) \quad 0.68, \\
1.08,1.48\end{array}$ \\
\hline $\begin{array}{l}3970.15 \\
3968.11\end{array}$ & 5 & $\begin{array}{r}5 \\
150\end{array}$ & V $E$ & $\begin{array}{l}25180.87 \\
25193.81\end{array}$ & $\begin{array}{l}c^{3} \mathrm{~F}_{4}-y{ }^{1} \mathrm{H}_{5}^{\circ} \\
a^{3} \mathrm{P}_{0}-z \mathrm{~F}_{1}^{\circ}\end{array}$ & (0) 0.34 & (0) 0.35 \\
\hline $\begin{array}{l}3960.37 \\
3951.968 \\
3948.00\end{array}$ & 10 & $\begin{array}{r}1 \\
500 \\
1\end{array}$ & V $E$ & $\begin{array}{l}25243.05 \\
25296.72 \\
25322.14\end{array}$ & $\begin{array}{l}a^{1} \mathrm{~F}_{3}-y^{3} \mathrm{H}^{\circ} \\
a^{3} \mathrm{P}_{2}-z^{3} \mathrm{D}_{3}^{\circ}\end{array}$ & (0) 1.17 & (0) $1.15 b$ \\
\hline $\begin{array}{l}\text { 3929. } 734 \\
3926.497 \\
3926.32\end{array}$ & $\begin{array}{l}2 \\
1\end{array}$ & $\begin{array}{r}50 \\
10 \\
5\end{array}$ & & $\begin{array}{l}25439.84 \\
25460.81 \\
25461.96\end{array}$ & $\begin{array}{l}a^{3}{ }^{3} \mathrm{P}_{1}-z^{3} \mathrm{D}_{1}^{\circ} \\
a^{3} \mathrm{P}_{2}-z^{5} \mathrm{D}_{2}^{\circ} \\
a^{1} \mathrm{H}_{5}-y^{3} \mathrm{G}_{5}^{\circ}\end{array}$ & $\begin{array}{l}\text { (1.21) } 0.30,1.45 \\
\text { (0) } 1.44\end{array}$ & $\begin{array}{l}(1.24) 0.24,1.48 \\
(0.18) 1.44 b\end{array}$ \\
\hline 3916.418 & 5 & 200 & V $E$ & 25526.33 & $a^{3} \mathrm{P}_{1}-z^{3} \mathrm{D}_{2}^{2}$ & $\begin{array}{c}(\mathbf{0}, 0.43) \quad 0.64, \\
1.02,1.49\end{array}$ & $\begin{array}{c}(\mathbf{0}, 0.40) \\
1.08,1.48\end{array}$ \\
\hline 3914. 333 & 15 & 250 & & 25539.93 & $a^{3} \mathrm{G}_{3}-z^{3} \mathrm{~F}_{2}^{\circ}$ & (0) 0.84 & (0) $0.83 b$ \\
\hline $\begin{array}{l}3907.52 \\
3903.27 \\
3899.140 \\
3896.155^{\circ} \\
3891.25\end{array}$ & $\begin{array}{c}10 \\
8 \\
20 ?\end{array}$ & $\begin{array}{c}3 \\
250 \\
200 \\
60+\mathrm{I} \\
4\end{array}$ & $\begin{array}{l}\mathrm{V} \\
\mathrm{V}\end{array}$ & $\begin{array}{l}25584.46 \\
25612.32 \\
25639.44 \\
25659.09 \\
25691.43\end{array}$ & $\begin{array}{l}b^{1} \mathrm{D}_{2}-y^{3} \mathrm{D}_{2}^{\circ} \\
a^{3} \mathrm{P}_{2}-z{ }^{5} \mathrm{D}_{3}^{\circ} \\
a^{3} \mathrm{G}_{4}-z{ }^{3} \mathrm{~F}_{3}^{\circ} \\
a^{3} \mathrm{P}_{0}-z z^{3} \mathrm{D}_{1}^{\circ} \\
b^{3} \mathrm{~F}_{3}-z^{3} \mathrm{G}_{3}^{\circ}\end{array}$ & $\begin{array}{l}\text { (0) } 1.35 \\
\text { (0) } 1.01 \\
\text { (0) } 0.27\end{array}$ & $\begin{array}{l}\text { (0) } 1.45 b \\
\text { (0) } 0.97 b \\
\text { (0) } 0.24\end{array}$ \\
\hline
\end{tabular}

See footnotes at end of table. 
TABLE 1.-Second spectrum of Vanadium-Continued

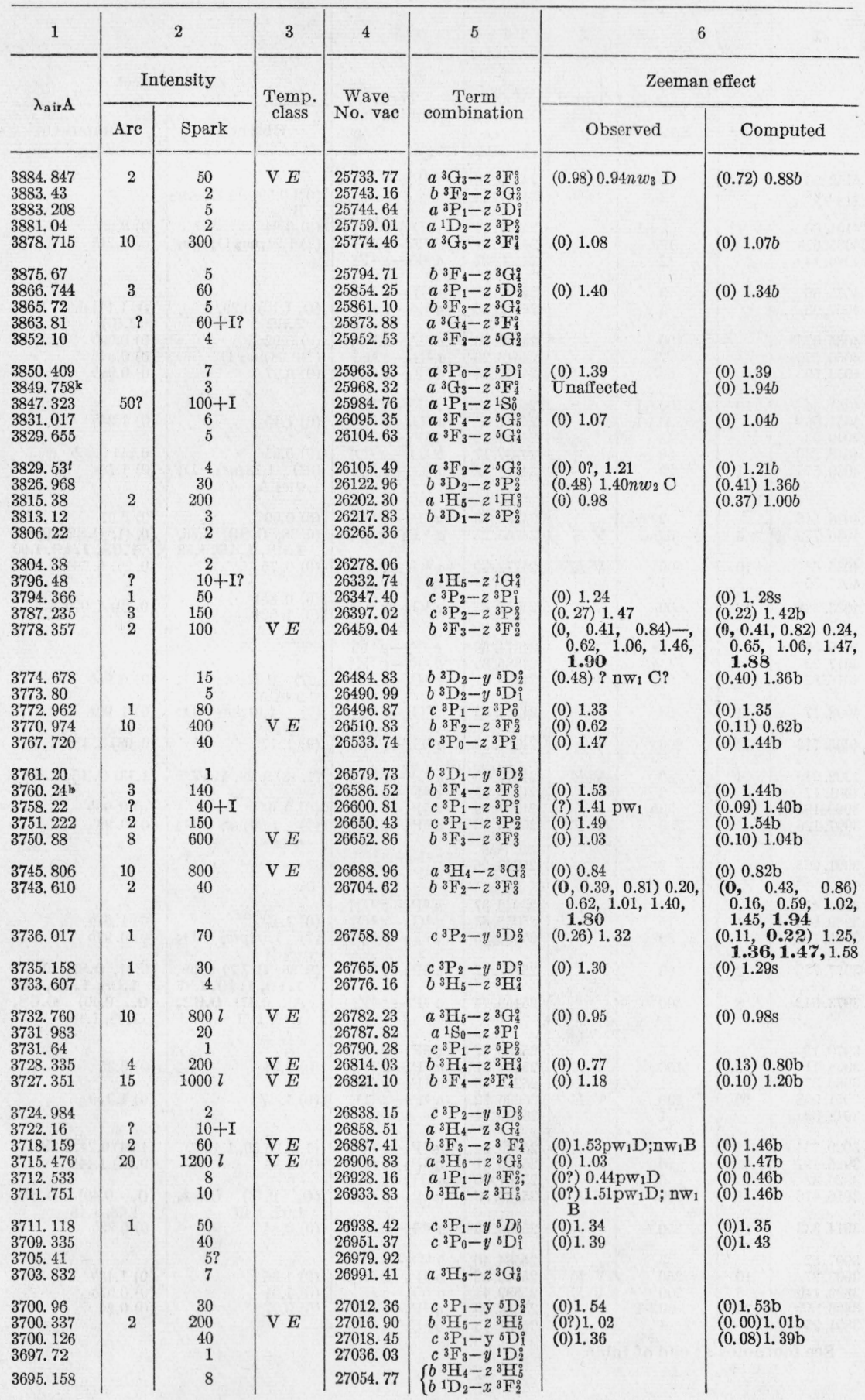

See footnotes at end of table. 
TABLE 1.-Second spectrum of Vanadium-Continued

\begin{tabular}{|c|c|c|c|c|c|c|c|}
\hline 1 & & 2 & 3 & 4 & 5 & 6 & \\
\hline \multirow{2}{*}{$\lambda_{\mathrm{s} \text { ir } \mathrm{A}}$} & \multicolumn{2}{|c|}{ Intensity } & \multirow{2}{*}{$\begin{array}{l}\text { Temp. } \\
\text { class }\end{array}$} & \multirow{2}{*}{$\begin{array}{l}\text { Wave } \\
\text { No. vac }\end{array}$} & \multirow{2}{*}{$\begin{array}{c}\text { Term } \\
\text { combination }\end{array}$} & \multicolumn{2}{|c|}{ Zeeman effect } \\
\hline & Arc & Spark & & & & Observed & Computed \\
\hline $\begin{array}{l}3690.70 \\
3674.691 \\
3669.410^{\mathrm{b}} \\
3661.383^{\mathrm{b}} \\
3658.266\end{array}$ & $\begin{array}{l}3 \\
2\end{array}$ & $\begin{array}{r}1 \\
30 \\
300 \\
200 \\
10\end{array}$ & V $E$ & $\begin{array}{l}27087.45 \\
27205.45 \\
27244.61 \\
27304.33 \\
27327.60\end{array}$ & $\begin{array}{l}a{ }^{1} \mathrm{~F}_{3}-w \\
a{ }^{3} \mathrm{D}_{3}^{\circ} \\
{ }^{1} \mathrm{~S}_{0}-\mathrm{y}{ }^{5} \mathrm{D}_{1}^{1} \\
b{ }^{3} \mathrm{H}_{6}-z \\
a{ }^{3} \mathrm{H}_{6}^{\circ} \\
{ }^{1} \mathrm{~F}_{3}-y \\
b^{3} \mathrm{H}^{5}-z{ }^{3} \mathrm{H}_{6}^{\circ}\end{array}$ & $\begin{array}{l}\text { (0) } 1.43 \\
\text { (0) } 1.11 \\
\text { (0) } 0.96 \\
\text { (0?) } 1.51 \mathrm{pw}_{3} \mathrm{D} ; n w_{3} \\
\text { A }\end{array}$ & $\begin{array}{l}\text { (0) } 1.43 \\
(0.05) 1.14 \mathrm{~b} \\
\text { (0) } 1.04 \mathrm{~b} \\
\text { (0) } 1.43 \mathrm{~b}\end{array}$ \\
\hline 3646.848 & & 7 & & 27413.16 & $b{ }^{1} \mathrm{D}_{2}-x{ }^{3} \mathrm{D}_{\mathrm{i}}$ & (0) $1.21 p w_{1} ; n w_{1}$ & (0) $1.17 b$ \\
\hline $\begin{array}{l}3645.905 \\
3634.13 \\
3632.126 \\
3631.482\end{array}$ & & $\begin{array}{r}30 \\
1 \\
15 \\
10\end{array}$ & & $\begin{array}{l}27420.25 \\
27509.09 \\
27524.27 \\
27529.15\end{array}$ & $\begin{array}{l}b^{3} \mathrm{P}_{1}-z{ }^{3} \mathrm{P}_{0}^{0} \\
b^{1} \mathrm{D}_{2}-x^{3} \mathrm{D}_{2} \\
b^{3} \mathrm{P}_{1}-z z^{3} \mathrm{P}_{1}^{0} \\
b^{3} \mathrm{P}_{0}-z z^{3} \mathrm{P}_{1}^{\circ}\end{array}$ & $\begin{array}{l}\text { (0) } 1.39 \\
\text { (0) } 1.41 \\
\begin{array}{l}\text { (0) } 1.38 \\
n w_{3} \mathrm{~A}\end{array} \quad p w_{2} \quad \mathrm{D} \text {; }\end{array}$ & $\begin{array}{l}\text { (0) } 1.40 \\
(0.04) 1.42 b \\
(0) 1.44\end{array}$ \\
\hline $\begin{array}{l}3627.713 \\
3626.64 \\
3625.608 \\
3623.03 \\
3622.289\end{array}$ & $\begin{array}{l}1 \\
1\end{array}$ & $\begin{array}{r}60 \\
1 \\
50 \\
1 \\
10\end{array}$ & & $\begin{array}{l}27557.75 \\
27565.90 \\
27573.75 \\
27593.37 \\
27599.01\end{array}$ & $\begin{array}{l}b^{3} \mathrm{P}_{2}-z{ }^{3} \mathrm{P}_{1}^{0} \\
b^{3} \mathrm{P}_{1}-z{ }^{3} \mathrm{P}_{2}^{0} \\
b^{3} \mathrm{P}_{0}-z{ }^{5} \mathrm{P}_{1}^{0} \\
a^{1} \mathrm{D}_{2}-y^{3} \mathrm{G}_{3}^{8}\end{array}$ & $\begin{array}{l}\text { (0) } 1.37 \\
\text { (0) } 1.45\end{array}$ & $\begin{array}{l}(0) 1.35 b \\
(0) 1.52 b\end{array}$ \\
\hline $\begin{array}{l}3621.203 \\
3620.496 \\
3618.924^{\mathrm{b}} \\
3611.58 \\
3608.32\end{array}$ & 2 & $\begin{array}{c}150 \\
20 \\
200 \\
10 h \\
1\end{array}$ & V $E$ & $\begin{array}{l}27607.29 \\
27612.68 \\
27624.68 \\
27680.85 \\
27705.85\end{array}$ & $\begin{array}{l}b^{3}{ }^{3} \mathrm{P}_{2}-z \\
b^{1}{ }^{3} \mathrm{P}_{2}^{\circ} \\
a^{1} \mathrm{P}_{1}-z{ }^{1} \mathrm{P}_{1}^{0} \\
{ }^{1} \mathrm{D}_{2}^{\circ} \\
c^{1} \mathrm{G}_{4}-w^{3} \mathrm{G}_{4}\end{array}$ & $\begin{array}{l}\text { (0) } 1.41 \\
\text { (0) } 1.01 \\
\text { (0) } 0.92 \\
\text { (0) } 1.31\end{array}$ & $\begin{array}{l}(0.18) 1.43 b \\
(0) 1.02 b \\
\text { (0) } 0.91 b\end{array}$ \\
\hline $\begin{array}{l}3607.30 \\
3604.375 \\
3602.94 \\
3598.72 \\
3597.41\end{array}$ & & $\begin{array}{l}1 \\
4 \\
3 h \\
3 \\
3\end{array}$ & & $\begin{array}{l}27713.69 \\
27736.18 \\
27747.22 \\
27779.76 \\
27789.88\end{array}$ & $\begin{array}{l}b^{3} \mathrm{P}_{1}-z{ }^{5} \mathrm{P}_{2}^{0} \\
b^{3} \mathrm{D}_{1}-z{ }^{1} \mathrm{~S}_{0}^{0}\end{array}$ & $\begin{array}{l}\text { (0) } 0.59 \\
\text { (?) } 1.22 \mathrm{nw}_{1} \mathrm{~A}\end{array}$ & (0) 0.58 \\
\hline $\begin{array}{l}3593.323^{\mathrm{b}} \\
3592.012^{\mathrm{b}} \\
3589.745^{\mathrm{b}} \\
3588.13 \\
3578.636\end{array}$ & $\begin{array}{l}15 \\
20 \\
25\end{array}$ & $\begin{array}{r}600 \\
800 \\
1000 \\
15 \\
15\end{array}$ & $\begin{array}{l}\mathrm{V} E \\
\mathrm{~V} E \\
\mathrm{~V} E\end{array}$ & $\begin{array}{l}27821.48 \\
27831.64 \\
27849.21 \\
27861.75 \\
27935.66\end{array}$ & $\begin{array}{l}a^{3} \mathrm{~F}_{4}-z^{5} \mathrm{~F}_{3}^{0} \\
a^{3} \mathrm{~F}_{3}-z^{5} \mathrm{~F}_{2}^{0} \\
a^{3} \mathrm{~F}_{2}-z^{5} \mathrm{~F}_{1}^{8} \\
b^{3} \mathrm{P}_{1}-y^{5} \mathrm{D}_{0}^{8} \\
b^{3} \mathrm{P}_{1}-y^{5} \mathrm{D}_{2}^{2}\end{array}$ & $\begin{array}{l}\text { (0?) } 1.14 \\
\text { (0) } 1.01 \\
\text { (0?) } 0.79 \\
\text { (0) } 1.40\end{array}$ & $\begin{array}{ll}\text { (0) } & 1.16 \mathrm{~s} \\
\text { (0) } & 1.00 \mathrm{~b} \\
(0) & 0.80 \mathrm{~b} \\
\text { (0) } & 1.40\end{array}$ \\
\hline $\begin{array}{l}3577.857 \mathrm{~d} \\
3577.644 \\
3577.220 \\
3574.340 \\
3573.557\end{array}$ & 1 & $\begin{array}{l}20+\mathrm{I} ? \\
3 \\
10 \\
60 \\
50\end{array}$ & & $\begin{array}{l}27941.74 \\
27943.41 \\
27946.72 \\
27969.24 \\
27975.36\end{array}$ & $\begin{array}{l}b^{3} \mathrm{P}_{1}-y^{5} \mathrm{D}_{1}^{i} \\
b^{1} \mathrm{G}_{4}-z^{3} \mathrm{H}_{4}^{4} \\
b^{3} \mathrm{P}^{0}-y^{5} \mathrm{D}_{1}^{i} \\
b^{3} \mathrm{P}_{2}-y^{5} \mathrm{D}_{2}^{2} \\
b^{3} \mathrm{P}_{2}-y^{5} \mathrm{D}_{1}^{8}\end{array}$ & $\begin{array}{l}\text { (?) } 1.28 \mathrm{pw}_{1} \mathrm{D} \\
\text { (0) } 1.45 \\
\text { (0) } 1.41 \\
\text { (0) } 1.34\end{array}$ & $\begin{array}{l}(0.03) 1.42 \mathrm{~b} \\
(0) 1.43 \\
(0.16) 1.42 b \\
\text { (0) } 1.33 \mathrm{~s}\end{array}$ \\
\hline 3566.177 & 10 & 200 & & 28033.26 & $a^{3} \mathrm{~F}_{2}-z^{5} \mathrm{~F}_{2}^{\circ}$ & $\begin{array}{c}(0.38,0.87) \\
0.61,1.04,1.49\end{array}$ & $\begin{array}{l}(0.43,0.86) 0.22, \\
0.65,1.08, \\
1.51\end{array}$ \\
\hline $\begin{array}{l}3563.71 \\
3560.594\end{array}$ & 4 & $\begin{array}{l}3 h \\
90\end{array}$ & & $\begin{array}{l}28052.66 \\
28077.21\end{array}$ & $\begin{array}{l}a^{3} \mathrm{~F}_{4}-z^{5} \mathrm{~F}_{4}^{0} \\
a^{3} \mathrm{~F}_{3}-z^{5} \mathrm{~F}_{2}^{0}\end{array}$ & $(0.50) 1.08 \mathrm{nw}_{2} \mathrm{C}$ & $\begin{array}{c}(0.20,0.40,0.60) \\
0.64,0.84, \mathbf{1 . 0 4}, \\
\mathbf{1 . 2 4}, 1.44,1.64\end{array}$ \\
\hline 3556.800 & 40 & 1500 & V $E$ & 28107.16 & ${ }^{3}{ }^{3} \mathrm{~F}_{4}-z^{3} \mathrm{D}_{3}^{\circ}$ & (0) 1.09 & (0) $1.07 b$ \\
\hline 3550.51 & & 3 & & 28156.95 & & & \\
\hline $\begin{array}{l}3549.030^{\circ} \\
3547.07 \\
3545.190 \mathrm{~g} \\
3542.480 \\
3541.341\end{array}$ & 30 & $\begin{array}{r}3 \\
5 \\
1000 \\
4 \\
50\end{array}$ & V $E$ & $\begin{array}{l}28168.70 \\
28184.26 \\
28199.20 \\
28220.78 \\
28229.85\end{array}$ & $\begin{array}{l}c^{3} \mathrm{P}_{1}-z^{1} \mathrm{~S}_{0}^{0} \\
b^{1} \mathrm{G}_{4}-z^{3} \mathrm{H}_{5}^{\circ} \\
a^{3} \mathrm{~F}_{3}-z^{3} \mathrm{D}_{2}^{0} \\
a^{1} \mathrm{D}_{2}-y^{3} \mathrm{~F}_{2}^{0} \\
a^{1} \mathrm{D}_{2}-y^{3} \mathrm{~F}_{3}^{0}\end{array}$ & $\begin{array}{l}\text { (0) } 0.95 ? \\
\text { (0) } 0.97 \\
\text { (0) } 0.98\end{array}$ & $\begin{array}{l}\text { (0) } 1.35 \\
(0) 0.96 s \\
(0) 0.96 b\end{array}$ \\
\hline 3538.238 & 3 & 50 & & 28254.61 & $a^{3} \mathrm{~F}_{4}-z^{5} \mathrm{~F}_{5}^{\circ}$ & (?) ${ }_{\mathrm{C}}^{1.75 \mathrm{pw}_{1} \mathrm{D} ; \mathrm{nw}_{1}}$ & (0) $1.76 b$ \\
\hline $\begin{array}{l}3534.14 \\
3532.285 \\
3531.48 \\
3530.765\end{array}$ & 20 & $\begin{array}{r}2 \\
20 \\
10 \\
500\end{array}$ & $\mathrm{~V} E$ & $\begin{array}{l}28287.37 \\
28302.23 \\
28308.68 \\
28314.41\end{array}$ & $\begin{array}{l}a{ }^{3} \mathrm{P}_{2}-z{ }^{3} \mathrm{~F}_{3}^{\circ} \\
a{ }^{1} \mathrm{~F}_{3}-y^{1} \mathrm{~F}_{3}^{0} \\
a^{3} \mathrm{~F}_{3}-z{ }^{3} \mathrm{~F}_{4}^{\circ} \\
a^{3} \mathrm{~F}_{2}-z{ }^{3} \mathrm{D}_{1}^{\circ}\end{array}$ & $\begin{array}{l}(0.20) 0.92 \\
(0,0.47) 0.21,0.62 \\
1.11\end{array}$ & $\begin{array}{l}(0.08) 0.96 b \\
(\mathbf{0}, 0.41) 0.24,0.65 \\
\text { 1.06 }\end{array}$ \\
\hline
\end{tabular}

See footnotes at end of table. 
TABLE 1.-Second spectrum of Vanadium-Continued

\begin{tabular}{|c|c|c|c|c|c|c|c|}
\hline 1 & & 2 & 3 & 4 & 5 & \multicolumn{2}{|c|}{6} \\
\hline \multirow{2}{*}{$\lambda_{\mathrm{a} \text { ir } \mathrm{A}}$} & \multicolumn{2}{|c|}{ Intensity } & \multirow{2}{*}{$\begin{array}{l}\text { Temp. } \\
\text { class }\end{array}$} & \multirow{2}{*}{$\begin{array}{l}\text { Wave } \\
\text { No. vac }\end{array}$} & \multirow{2}{*}{$\begin{array}{c}\text { Term } \\
\text { combination }\end{array}$} & \multicolumn{2}{|c|}{ Zeeman effect } \\
\hline & Arc & Spark & & & & Observed & Computed \\
\hline $\begin{array}{l}3530.45 \\
3527.867\end{array}$ & & 10 & & 28316.94 & $a^{3} \mathrm{D}_{1}-z{ }^{3} \mathrm{P}_{0}^{0}$ & (0) 0.52 & (0) 0.49 \\
\hline $\begin{array}{l}3527.867 \\
3524.713\end{array}$ & 10 & $\begin{array}{r}10 \\
200\end{array}$ & VE & $\begin{array}{l}28337.67 \\
28363.02\end{array}$ & $\begin{array}{l}b{ }^{3} \mathrm{H}_{4}-y^{3} \mathrm{G}_{3}^{8} \\
a^{3} \mathrm{~F}_{3}-z^{3} \mathrm{D}_{3}^{8}\end{array}$ & $2 p ; n w 3$ & \\
\hline 3521.836 & 2 & 90 & $\mathrm{~V} E$ & 28386.19 & $a^{3} \mathrm{D}_{3}-z^{3} \mathrm{P}_{2}^{3}$ & (0)1.04pw 1 D; $\mathrm{nw}_{1}$ & (0) $1.12 b$ \\
\hline 3520.547 & 1 & 15 & & 28396.58 & $a^{3} \mathrm{D}_{2}-2{ }^{3} \mathrm{P}_{1}^{\circ}$ & (?) $0.74 \mathrm{pw}_{2} \mathrm{D} ; \mathrm{nw}_{2}$ & (0) $0.82 \mathrm{~s}$ \\
\hline 3520.022 & 5 & 120 & VE & 28400.98 & $a^{3} \mathrm{~F}_{2}-z{ }^{3} \mathrm{D}_{2}^{\circ}$ & $(0.27,0.90) \quad 0.19$ & $(0.43,0.86) 0.22$, \\
\hline $\begin{array}{l}3517.298 \\
3516.00\end{array}$ & 40 & $\begin{array}{r}800 \\
5\end{array}$ & $\mathrm{~V} E$ & $\begin{array}{l}28422.82 \\
28433.31\end{array}$ & $\begin{array}{l}a^{3} \mathrm{~F}_{4}-z^{5} \mathrm{D}_{3}^{3} \\
a^{3} \mathrm{~F}_{4}-z^{5} \mathrm{D}^{3}\end{array}$ & 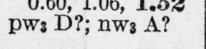 & $0.00,1.08,1.01$ \\
\hline $\begin{array}{l}3514.422 \\
3513.877\end{array}$ & & $\begin{array}{l}0 \\
20 \\
15\end{array}$ & & $\begin{array}{l}28446.08 \\
28450.49\end{array}$ & $\begin{array}{l}a^{3} \mathrm{D}_{2}-z^{3} \mathrm{P}_{2}^{4} \\
b^{3} \mathrm{H}_{3}-y^{3} \mathrm{G}_{4}^{\circ}\end{array}$ & $\begin{array}{l}(0.66) 1.43 \mathrm{nw}_{3} \mathrm{C} \\
(0) 0.93\end{array}$ & $\begin{array}{l}(0.63) 1.30 b \\
(0) 0.97 s\end{array}$ \\
\hline $\begin{array}{l}3512.13 \\
3511.42 \\
3509.684\end{array}$ & & $\begin{array}{l}3 \\
3 \\
2\end{array}$ & & $\begin{array}{l}28464.64 \\
28470.39 \\
28484.48\end{array}$ & $\begin{array}{l}a^{1} \mathrm{~F}_{3}-x^{3} \mathrm{G}_{4}^{0} \\
a^{3} \mathrm{D}_{1}-z^{3} \mathrm{P}_{2}^{0}\end{array}$ & (0) 1.09 & (0) $1.10 b$ \\
\hline $\begin{array}{l}3509.024 \\
3507.534\end{array}$ & $\begin{array}{l}1 \\
1\end{array}$ & $\begin{array}{l}40 \\
20\end{array}$ & & $\begin{array}{l}28489.83 \\
28501.94\end{array}$ & $\begin{array}{l}b^{3} \mathrm{H}_{6}-y^{3} \mathrm{G}_{5}^{\circ} \\
a^{1} \mathrm{P}_{1}-y^{3} \mathrm{D}_{2}^{2}\end{array}$ & $\begin{array}{l}\text { (0) } 1.06 \\
\text { (0) } 1.17\end{array}$ & $\begin{array}{l}\text { (0) } 0.94 b \\
\text { (0) } 1.18 b\end{array}$ \\
\hline $\begin{array}{l}3506.57 \\
3504.432\end{array}$ & 20 & $\begin{array}{r}7 \\
400\end{array}$ & & $\begin{array}{l}28509.77 \\
28527.16\end{array}$ & $\begin{array}{l}a{ }^{1} \mathrm{~F}_{3}-x^{3} \mathrm{G}_{3}^{\circ} \\
a^{3} \mathrm{~F}_{3}-z^{5} \mathrm{D}_{2}^{\circ}\end{array}$ & $\begin{array}{l}(0.47) ? \mathrm{nw}_{2} \mathrm{D} \\
(?) \quad 0.29, \quad 0.67, \\
1.02, \quad 1.36, \quad 1.72\end{array}$ & $\begin{array}{l}(0.39) 0.90 b \\
(\mathbf{0}, 0.35,0.70) \\
\text { 0.34, } 0.69,1.04 \text {, }\end{array}$ \\
\hline 3499.823 & 2 & 20 & & 28564.73 & $a^{3} \mathrm{~F}_{2}-z^{3} \mathrm{D}_{3}^{\circ}$ & $\begin{array}{c}\mathrm{pw}_{3} \\
(\mathbf{0}, 0.66,1.31) \\
0.65,1.30,1.95, \\
\mathbf{2 . 6 3}\end{array}$ & 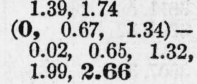 \\
\hline $\begin{array}{l}3497.39 \\
3497.031\end{array}$ & 2 & $\begin{array}{r}4 \\
200\end{array}$ & & $\begin{array}{l}28584.60 \\
28587.54\end{array}$ & $\begin{array}{l}b^{3}{ }^{3} \mathrm{D}_{2}-y^{3} \mathrm{~F}_{2}^{\circ} \\
a^{1} \mathrm{D}_{2}-z^{1} \mathrm{~F}_{3}^{\circ}\end{array}$ & (0) 0.96 & (0) $0.92 b$ \\
\hline 3493.163 & 8 & 150 & & 28619.19 & $a^{3} \mathrm{~F}_{2}-z{ }^{5} \mathrm{D}_{1}^{\circ}$ & $(0,0.75) \quad 0,0.68$ & $(0, \quad 0.74)-0.09$ \\
\hline 3489.947 & 1 & 20 & & 28645.56 & $b^{3} \mathrm{D}_{3}-y^{3} \mathrm{~F}_{4}^{\circ}$ & (0) $0.88 \mathrm{pw}_{1} \quad \mathrm{D}$; & (0) 0.948 \\
\hline 3485.916 & 10 & 250 & & 28678.69 & $a^{3} \mathrm{~F}_{3}-z^{5} \mathrm{D}_{3}^{\circ}$ & $(1.07) \quad 1.31 \mathrm{pw}_{3} \quad \mathrm{~B}$; & (1.11) $1.26 b$ \\
\hline $\begin{array}{l}3484.65 \\
3484.32\end{array}$ & & $\begin{array}{l}3 \\
2\end{array}$ & & $\begin{array}{l}28689.11 \\
28691.82\end{array}$ & $\begin{array}{l}a^{3} \mathrm{~F}_{3}-z{ }^{5} \mathrm{D}_{4}^{\circ} \\
a^{1} \mathrm{H}_{5}-y^{3} \mathrm{H}_{4}^{\circ}\end{array}$ & & \\
\hline 3479.837 & 5 & 80 & & 28728.78 & $a^{3} \mathrm{~F}_{2}-z^{5} \mathrm{D}_{2}^{\circ}$ & $(0.72, \mathbf{1 . 5 0}) 0,0.65$ & $(0.74,1.48)-0.09$ \\
\hline 3478.961 & & 6 & & 28736.02 & $b{ }^{1} \mathrm{D}_{2}-w{ }^{3} \mathrm{D}_{3}^{\circ}$ & (0) $1.75 \mathrm{pw}_{2} \mathrm{D}$ & $\begin{array}{l}\text { (0) } 1.75 b \\
\text { (.6.39, } 2.13 \\
\text { (1) }\end{array}$ \\
\hline $\begin{array}{l}3477.514 \\
3476.252 \\
3470.263\end{array}$ & $\begin{array}{l}2 \\
1 \\
1\end{array}$ & $\begin{array}{l}40 \\
20 \\
20\end{array}$ & & $\begin{array}{l}28747.97 \\
28758.41 \\
28808.04\end{array}$ & $\begin{array}{c}a^{3} \mathrm{D}_{3}-y{ }^{5} \mathrm{D}_{2}^{\circ} \\
a^{3} \mathrm{D}_{1}-y^{5} \mathrm{D}_{0}^{\circ} \\
a^{3} \mathrm{D}_{2}-y^{5} \mathrm{D}_{2}^{\circ}\end{array}$ & $\begin{array}{l}\text { (0) } 1.08 \\
(0) 0.49 \\
(0.64) 1.30 \mathrm{nw}_{2} \mathrm{C}\end{array}$ & $\begin{array}{l}\text { (0) } 1.13 b \\
\text { (0) } 0.49 \\
(0.61) 1.30 \mathrm{~b}\end{array}$ \\
\hline $\begin{array}{l}3469.528 \\
3467.33\end{array}$ & 2 & $\begin{array}{r}50 \\
2\end{array}$ & & $\begin{array}{l}28814.14 \\
28832.41\end{array}$ & $\begin{array}{l}a^{3} \mathrm{D}_{2}-y={ }^{5} \mathrm{D}_{1}^{\circ} \\
a^{3} \mathrm{D}_{1}-y^{5} \mathrm{D}_{2}^{5}\end{array}$ & (?) $0.95 \mathrm{nw}_{2} \mathrm{~A}$ & (0) $0.98 \mathrm{~b}$ \\
\hline $\begin{array}{l}3466.59 \\
3465.25\end{array}$ & 1 & 20 & & $\begin{array}{l}28838.56 \\
28849.72\end{array}$ & $a^{3} \mathrm{D}_{1}-y^{3} \mathrm{D}_{1}^{2}$ & $(0.96) 0.47,1.45$ & $(0.94) 0.49,1.43$ \\
\hline 3464.17 & & 6 & & 28858.71 & $c^{3} \mathrm{P}_{2}-y^{3} \mathrm{~F}_{2}^{0}$ & (1.35) ?nw2 D & $(1.31) 1.00 \mathrm{~b}$ \\
\hline $\begin{array}{l}3463.831 \\
3463.079 \\
3461.580\end{array}$ & & $\begin{array}{l}4 \\
4 \\
5\end{array}$ & & $\begin{array}{l}28861.53 \\
28867.80 \\
28880.30\end{array}$ & $\begin{array}{l}a^{1} \mathrm{H}_{5}-y{ }^{3} \mathrm{H}_{6}^{\circ} \\
c^{3} \mathrm{P}_{2}-y{ }^{3} \mathrm{~F}_{3}^{6} \\
a^{3} \mathrm{~F}_{2}-z \\
{ }^{3} \mathrm{D}_{3}^{\circ}\end{array}$ & & \\
\hline 3457.153 & 3 & 300 & & 28917.28 & $a^{1} \mathrm{D}_{2}-z^{1} \mathrm{D}_{2}^{8}$ & (0) 0.96 & $(0.16) 0.98 b$ \\
\hline 3453.78 & & 1 & & 28945.52 & $b^{3} \mathrm{D}_{3}-2^{1} \mathrm{~F}_{3}^{\circ}$ & & \\
\hline 3453.087 & 1 & 90 & & 28951.33 & $b^{3} \mathrm{D}_{2}-z^{1} \mathrm{~F}^{2}$ & (0?) $0.71 \mathrm{pw}_{2} \mathrm{D}$; & (0) $0.69 b$ \\
\hline $\begin{array}{l}3451.046 \\
3448.69 \\
3438.232 \\
3436.393\end{array}$ & & $\begin{array}{r}12 \\
1 \\
2 \\
2\end{array}$ & & $\begin{array}{l}28968.45 \\
28988.24 \\
29076.40 \\
29091.97\end{array}$ & $\begin{array}{c}b{ }^{3} \mathrm{H}_{4}-y{ }^{3} \mathrm{~F}_{3} \\
b{ }^{3} \mathrm{H}_{5}-y{ }^{3} \mathrm{~F}_{4} \\
b^{3} \mathrm{P}_{1}-z{ }^{1} \mathrm{~S}_{0}\end{array}$ & (0) 1.41 & (0) 1.40 \\
\hline $\begin{array}{l}\text { 3435. } 38 \\
3424.46 \\
3434.024 \text { h } \\
3433.767 \\
3422.259\end{array}$ & & $\begin{array}{l}7 \\
1 \\
4 \\
3 \\
3 h\end{array}$ & & $\begin{array}{l}29100.55 \\
29108.34 \\
29112.04 \\
29114.22 \\
29212.12\end{array}$ & $\begin{array}{c}b{ }^{3} \mathrm{D}_{3}-z^{1} \mathrm{G}_{4} \\
b^{3} \mathrm{D}_{3}-2^{5} \mathrm{~S}_{2}^{2} \\
c^{3} \mathrm{P}_{1}-y^{3} \mathrm{~F}_{2}^{2} \\
b^{3} \mathrm{D}_{2}-z^{5} \mathrm{~S}_{2}^{2}\end{array}$ & $\begin{array}{l}(0.12)-? \\
\text { (0) } 1.48\end{array}$ & (0) $0.27 b$ \\
\hline
\end{tabular}

See footnotes at end of table. 
TABLE 1.-Second spectrum of Vanadium-Continued

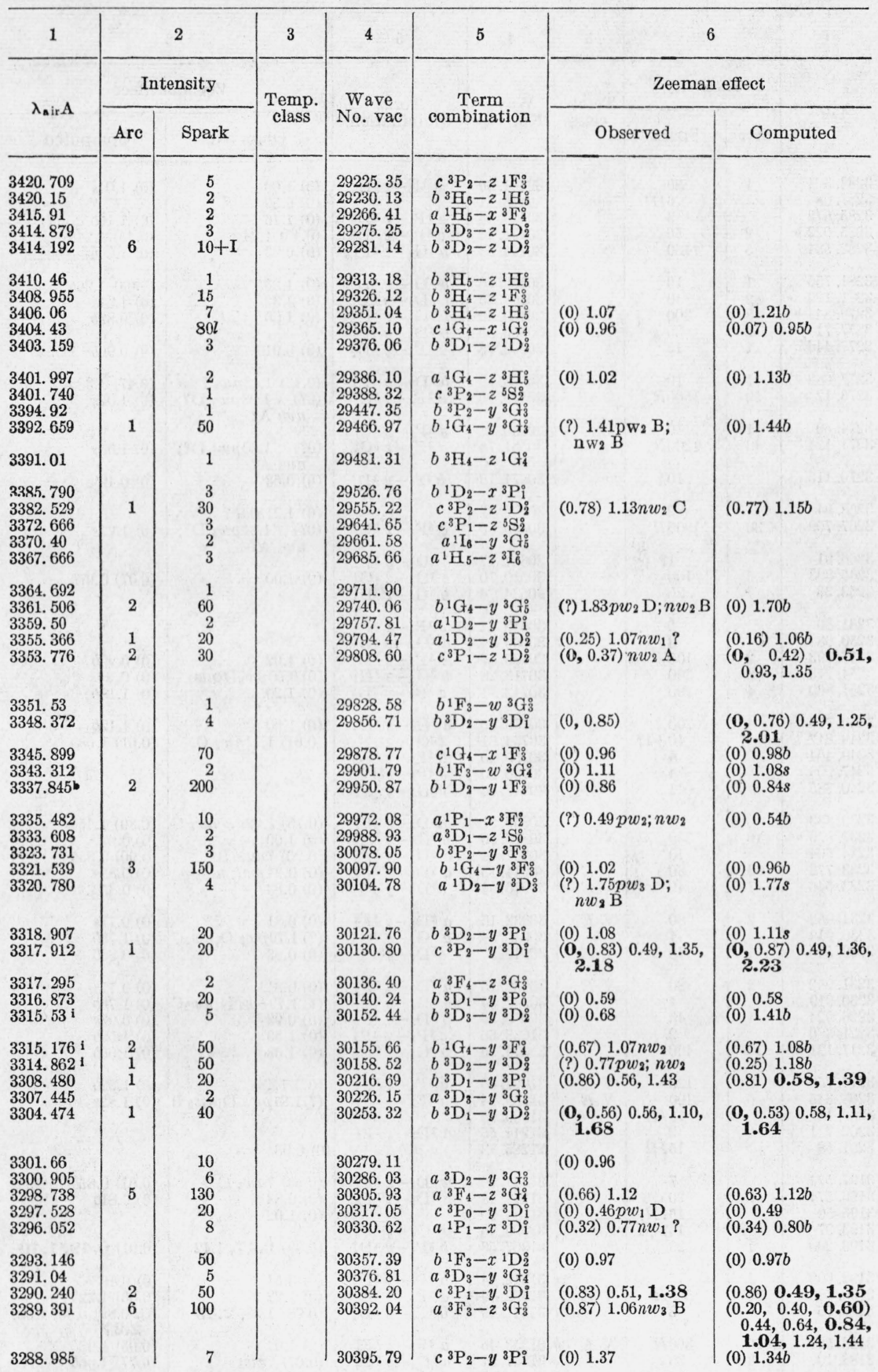

See footnotes at end of table. 
TABLE 1.-Second spectrum of Vanadium-Continued

\begin{tabular}{|c|c|c|c|c|c|c|c|}
\hline \multirow{3}{*}{$\lambda_{\mathrm{sir}} \mathrm{A}$} & \multicolumn{2}{|c|}{2} & 3 & 4 & 5 & \multicolumn{2}{|c|}{6} \\
\hline & \multicolumn{2}{|c|}{ Intensity } & \multirow{2}{*}{$\begin{array}{l}\text { Temp. } \\
\text { class }\end{array}$} & \multirow{2}{*}{$\begin{array}{l}\text { Wave } \\
\text { No. vac }\end{array}$} & \multirow{2}{*}{$\begin{array}{c}\text { Term } \\
\text { combination }\end{array}$} & \multicolumn{2}{|c|}{ Zeeman effect } \\
\hline & Arc & Spark & & & & Observed & Computed \\
\hline $\begin{array}{l}3288.324 \\
3287.08 \\
3285.672 \\
3285.022 \text { b } \\
3282.534\end{array}$ & $\begin{array}{l}2 \\
5\end{array}$ & $\begin{array}{l}20 \\
6 H l \\
3 \\
50 \\
150\end{array}$ & & $\begin{array}{l}30401.90 \\
30413.41 \\
30426.44 \\
30432.46 \\
30455.53\end{array}$ & $\begin{array}{l}a{ }^{1} \mathrm{I}_{6}-z{ }^{1} \mathrm{H}_{5}^{\circ} \\
a{ }^{1} \mathrm{P}_{1}-x \\
{ }^{3} \mathrm{D}_{2}^{\circ} \\
{ }^{3} \mathrm{P}_{2}-y \\
b^{1} \mathrm{G}_{4}-z z^{1} \mathrm{D}_{3}^{\circ}\end{array}$ & $\begin{array}{l}\text { (0) } 1.00 \\
\text { (0) } 0.59 \\
\text { (0) } 1.16 \\
\text { (0.45) } 1.24 \\
\text { (0) } 0.93\end{array}$ & $\begin{array}{l}\text { (0) } 1.01 \mathrm{~s} \\
\text { (0) } 1.16 \mathrm{~b} \\
(0.45) 1.24 \mathrm{~b} \\
\text { (0) } 1.00 \mathrm{~b}\end{array}$ \\
\hline $\begin{array}{l}3281.755 \\
3281.120 \\
3279.844 \text { b } \\
3277.71 \\
3277.448\end{array}$ & $\begin{array}{l}1 \\
2 \\
8 \\
2 \\
1\end{array}$ & $\begin{array}{r}10 \\
40 \\
300 \\
30 \\
15\end{array}$ & & $\begin{array}{l}30462.76 \\
30468.65 \\
30480.50 \\
30500.35 \\
30502.78\end{array}$ & $\begin{array}{l}b{ }^{3} \mathrm{D}_{3}-y{ }^{3} \mathrm{D}_{3}^{\circ} \\
b^{3} \mathrm{D}_{2}-y \\
b^{1} \mathrm{G}_{4}-z \mathrm{D}_{3}^{\circ} \mathrm{H}_{5}^{\circ} \\
b^{3} \mathrm{D}_{3}-y{ }^{3} \mathrm{P}_{2}^{\circ} \\
a^{1} \mathrm{P}_{3}-y{ }^{1} \mathrm{D}_{2}^{\circ}\end{array}$ & $\begin{array}{l}\text { (0) } 1.26 \\
\text { (0) } 1.32 \\
\text { (0) } 1.00 \\
\text { (0) } 0.91\end{array}$ & $\begin{array}{l}\text { (0.03) } 1.26 b \\
\text { (0) } 1.31 s \\
\text { (0) } 0.89 b \\
\text { (0) } 0.96 b\end{array}$ \\
\hline $\begin{array}{l}3277.082 \\
3276.12\end{array}$ & $\begin{array}{r}1 \\
50\end{array}$ & $\begin{array}{l}10 \\
1500 R\end{array}$ & & $\begin{array}{l}30506.19 \\
30515.15\end{array}$ & $\begin{array}{l}b^{3} \mathrm{D}_{2}-y{ }^{3} \mathrm{P}_{2}^{\circ} \\
a^{3} \mathrm{~F}_{4}-z^{3} \mathrm{G}_{5}^{\circ}\end{array}$ & $\begin{array}{l}(0.43) 1.43 n w_{1} ? \\
(0 ?) \quad 1.08 p w_{3} \mathrm{D} ?\end{array}$ & $\begin{array}{l}\text { (0.47) } 1.38 b \\
\text { (0) } 1.07 s\end{array}$ \\
\hline $\begin{array}{l}3274.50 \\
3271.124\end{array}$ & 40 & $\begin{array}{c}10 \\
1200 R\end{array}$ & & $\begin{array}{l}30530.24 \\
30561.75\end{array}$ & $\begin{array}{l}a^{1} \mathrm{P}_{1}-z{ }^{1} \mathrm{P}_{1}^{\circ} \\
a^{3} \mathrm{~F}_{3}-z^{3} \mathrm{G}_{4}^{2}\end{array}$ & (0?) $1.00 \mathrm{pw}_{3} \mathrm{D} ?$ & (0) $1.00 \mathrm{~s}$ \\
\hline 3270.115 & & 10 & & 30571.18 & $a^{1} \mathrm{~S}_{0}-y{ }^{3} \mathrm{D}_{1}^{\circ}$ & $\begin{array}{l}n w_{3} \mathrm{~A} ? \\
(0) 0.53\end{array}$ & (0) 0.49 \\
\hline $\begin{array}{l}3268.94 \\
3267.709\end{array}$ & 30 & $\stackrel{2}{1000 R}$ & & $\begin{array}{l}30582.17 \\
30593.69\end{array}$ & $a^{3} \mathrm{~F}_{2}-z^{3} \mathrm{G}_{3}^{\circ}$ & $\begin{array}{l}\text { (0) } 1.21 n w_{1} ? \\
\text { (0?) } 1.24 p w_{3} \mathrm{D} ? ;\end{array}$ & (0) $1.22 \mathrm{~s}$ \\
\hline $\begin{array}{l}3266.91 \\
3265.893 \\
3263.33\end{array}$ & $\begin{array}{l}5 \\
?\end{array}$ & $\begin{array}{c}1 ? \\
100 \\
20\end{array}$ & & $\begin{array}{l}30601.17 \\
30610.70 \\
30634.74\end{array}$ & $\begin{array}{l}b^{3} \mathrm{D}_{1}-y{ }^{3} \mathrm{P}_{2}^{\circ} \\
b^{1} \mathrm{G}_{4}-z \mathrm{G}_{4}^{1} \\
b^{3} \mathrm{G}_{4}-z^{3} \mathrm{H}_{4}^{\circ}\end{array}$ & (0) 1.00 & $(0.07) 0.97 b$ \\
\hline $\begin{array}{l}3261.80 \\
3259.684 \\
3257.893 \\
3254.773 \\
3251.869\end{array}$ & $\begin{array}{l}2 \\
8 \\
4\end{array}$ & $\begin{array}{r}5 \\
\mathbf{3} \\
100 \\
300 \\
200\end{array}$ & & $\begin{array}{l}30649.11 \\
30669.01 \\
30685.87 \\
30715.28 \\
30742.71\end{array}$ & $\begin{array}{l}c^{3} \mathrm{P}_{1}-y{ }^{3} \mathrm{P}_{1}^{0} \\
a^{1} \mathrm{G}_{4}-y^{3} \mathrm{G}_{3}^{0} \\
c^{3} \mathrm{P}_{1}-y y^{3} \mathrm{D}_{2}^{9} \\
b^{3} \mathrm{G}_{3}-2 z^{3} \mathrm{H}_{4}^{0} \\
c^{3} \mathrm{P}_{2}-y^{3} \mathrm{D}_{3}^{3}\end{array}$ & $\begin{array}{l}\text { (0) } 1.02 \\
\text { (0) } 0.90 p w_{2} \mathrm{D} ; n w_{2} \mathrm{~A} \\
\text { (0) } 1.20\end{array}$ & $\begin{array}{l}\text { (0) } 0.99 b \\
\text { (0) } 0.84 \mathrm{~s} \\
\text { (0) } 1.18 b\end{array}$ \\
\hline $\begin{array}{l}3250.775 \\
3249.617 \\
3249.464 \\
3247.908 \\
3240.785\end{array}$ & $\stackrel{4}{?}$ & $\begin{array}{c}200 \\
40+\mathrm{I} ? \\
4 \\
4 \\
1\end{array}$ & & $\begin{array}{l}30753.06 \\
30764.01 \\
30765.46 \\
30780.20 \\
30847.85\end{array}$ & $\begin{array}{l}a^{1}{ }^{1} \mathrm{H}_{3}-y^{1} \mathrm{G}_{4}^{\circ} \\
b^{3} \mathrm{G}_{5}-z^{3} \mathrm{H}_{5}^{\circ} \\
b^{3} \mathrm{P}_{2}-z^{1} \mathrm{D}_{2} \\
c^{3} \mathrm{P}_{2}-y^{3} \mathrm{P}_{2} \\
a^{3} \mathrm{D}_{3}-y^{3} \mathrm{~F}_{2}^{\circ}\end{array}$ & $\begin{array}{l}\text { (0) } 1.00 \\
(0.61) 1.11 n w_{2} \mathrm{C}\end{array}$ & $\begin{array}{l}\text { (0) } 1.12 b \\
(0.61) 1.08 b\end{array}$ \\
\hline $\begin{array}{l}3239.833 \\
3237.876 \text { b } \\
3234.504 \\
3233.772 \\
3233.546\end{array}$ & $\begin{array}{r}10 \\
3 \\
1\end{array}$ & $\begin{array}{r}8 \\
350 \\
10 \\
80 \\
40\end{array}$ & $\begin{array}{l}\text { V } E \\
\text { V } \frac{E}{E}\end{array}$ & $\begin{array}{l}30856.91 \\
30875.56 \\
30907.74 \\
30914.75 \\
30916.91\end{array}$ & $\begin{array}{c}a^{3} \mathrm{D}_{3}-y^{3} \mathrm{~F}_{3}^{\circ} \\
b^{3} \mathrm{G}_{4}-z^{3} \mathrm{H}_{5}^{0} \\
a^{3} \mathrm{D}_{2}-y^{3} \mathrm{~F}_{2}^{2} \\
a^{3} \mathrm{D}_{3}-y^{3} \mathrm{~F}_{4}^{\circ} \\
a^{3} \mathrm{D}_{2}-y^{3} \mathrm{~F}_{3}^{\circ}\end{array}$ & $\begin{array}{l}(0.76) 1.02 n w n w_{3} \mathrm{C} \\
(0) 1.00 \\
(0.96) \text { ? } n w_{3} \mathrm{~B} \text { ? } \\
\text { (0) } 0.94 p w_{1} ; n w_{1} \\
\text { (0) } 0.83\end{array}$ & $\begin{array}{l}(0.80) 1.15 b \\
(0) 0.97 b \\
(0.90) 0.88 b \\
\text { (0) } 0.82 s \\
\text { (0) } 0.85 b\end{array}$ \\
\hline $\begin{array}{l}3231.952 \\
3230.919 \\
3233.546\end{array}$ & 2 & $\begin{array}{r}80 \\
4 \\
40\end{array}$ & $\begin{array}{l}\text { V } E \\
\text { V } E\end{array}$ & $\begin{array}{l}30932.15 \\
30942.04 \\
30916.91\end{array}$ & $\begin{array}{c}a^{3} \mathrm{D}_{1}-y{ }^{3} \mathrm{~F}_{2}^{\circ} \\
a^{1} \mathrm{G}_{4}-y^{3} \mathrm{G}_{5}^{\circ} \\
a^{3} \mathrm{D}_{2}-y^{3} \mathrm{~F}_{3}^{\circ}\end{array}$ & $\begin{array}{l}\text { (0) } 0.81 \\
\text { (?) } 1.79 p w_{2} \mathrm{D} ; n w_{2} \mathrm{C} \\
\text { (0) } 0.83\end{array}$ & $\begin{array}{l}\text { (0) } 0.77 s \\
\text { (0) } 1.76 b \\
\text { (0) } 0.85 b\end{array}$ \\
\hline $\begin{array}{l}3231.952 \\
3230.919 \\
3226.924 \\
3221.380 \\
3217.121 \text { b }\end{array}$ & $\begin{array}{r}2 \\
1 \\
15\end{array}$ & $\begin{array}{r}80 \\
4 \\
40 \\
2 \\
400\end{array}$ & V $E$ & $\begin{array}{l}30932.15 \\
30942.04 \\
30980.35 \\
31033.66 \\
31074.75\end{array}$ & $\begin{array}{l}a^{3} \mathrm{D}_{1}-y^{3} \mathrm{~F}_{2}^{0} \\
a^{1} \mathrm{G}_{4}-y^{3} \mathrm{G}_{5}^{\circ} \\
b^{1} \mathrm{D}_{2}-y^{1} \mathrm{P}_{1}^{0} \\
c^{3} \mathrm{P}_{1}-y^{3} \mathrm{P}_{2}^{0} \\
b^{3} \mathrm{G}_{5}-z^{3} \mathrm{H}_{6}^{\circ}\end{array}$ & $\begin{array}{l}\text { (0) } 0.81 \\
\text { (?) } 1.79 p w_{2} \mathrm{D} ; n w_{2} \mathrm{C} \\
\text { (0) } 0.93 \\
\text { (0) } 1.50 \\
\text { (0) } 1.08\end{array}$ & $\begin{array}{l}\text { (0) } 0.77 s \\
\text { (0) } 1.76 b \\
\text { (0) } 0.93 s \\
\text { (0) } 1.59 b \\
\text { (0) } 1.06 b\end{array}$ \\
\hline $\begin{array}{l}3214.750 \\
3208.345 \\
3206.16 \\
3202.711 \\
3201.58\end{array}$ & $\begin{array}{l}6 \\
6\end{array}$ & $\begin{array}{l}120 \\
100 \\
15 H l \\
2 \\
15 H l\end{array}$ & $\begin{array}{l}\mathrm{V} \\
\mathrm{V} \\
E\end{array}$ & $\begin{array}{l}31097.66 \\
31159.74 \\
31180.98 \\
31214.55 \\
21225.58\end{array}$ & $\begin{array}{l}a^{3} \mathrm{~F}_{4}-z^{3} \mathrm{~F}_{3}^{\circ} \\
a^{3} \mathrm{~F}_{3}-z^{3} \mathrm{~F}_{2}^{\circ} \\
a^{3} \mathrm{D}_{3}-z^{1} \mathrm{~F}_{3}^{\circ}\end{array}$ & $\begin{array}{l}\text { (0?) } 1.46 \\
\text { (?) } 1.81 p w_{3} \mathrm{D} ; n w_{2} \mathrm{~B} \\
\text { (0) } 0.93\end{array}$ & $\begin{array}{l}\text { (0) } 1.52 b \\
\text { (0) } 1.828\end{array}$ \\
\hline $\begin{array}{l}3197.574 \\
3196.574 \\
3195.50 \\
3193.97 \\
3193.200\end{array}$ & 1 & $\begin{array}{l}7 \\
20 \\
15 H l \\
10 ?+\mathrm{I} \\
20\end{array}$ & & $\begin{array}{l}31264.70 \\
31274.48 \\
31284.99 \\
31299.98 \\
31307.53\end{array}$ & $\begin{array}{l}a{ }^{1} \mathrm{D}_{2}-x^{3} \mathrm{~F}_{2}^{\circ} \\
a^{3} \mathrm{D}_{2}-z^{1} \mathrm{~F}_{3}^{\circ} \\
a^{1} \mathrm{G}_{4}-y^{3} \mathrm{~F}_{3}^{\circ} \\
b^{3} \mathrm{P}_{1}-y^{3} \mathrm{D}_{1}^{\circ}\end{array}$ & $\begin{array}{l}(0.60) ? n w_{1} \mathrm{C} \\
(0) 0.83 \\
(0) 1.02 \\
(0.89) \mathbf{0 . 4 7}, 1.42\end{array}$ & $\begin{array}{l}(0.61) 0.85 b \\
(0) 0.81 b \\
(0.91) 0.49,1.40\end{array}$ \\
\hline $\begin{array}{l}3192.699 \\
3190.686 \text { b } \\
3189.76\end{array}$ & $\begin{array}{r}1 \\
30\end{array}$ & $\begin{array}{c}15 \\
500 R \\
3\end{array}$ & V $E$ & $\begin{array}{l}31312.44 \\
31332.19 \\
31341.29\end{array}$ & $\begin{array}{l}b^{3} \mathrm{P}_{0}-y^{3} \mathrm{Di}^{3} \\
a^{3} \mathrm{~F}_{4}-z^{3} \mathrm{~F}^{2} \\
b^{3} \mathrm{P}_{2}-y^{3} \mathrm{Di}^{3}\end{array}$ & $\begin{array}{l}\text { (0) } 0.54 \\
\text { (0) } 1.22 \\
\text { (0.98) } 1.38, \mathbf{2 . 3 9}\end{array}$ & $\begin{array}{l}\text { (0) } 0.49 \\
(0.00) 1.22 b \\
(\mathbf{0}, 0.89) 0.49,1.38 \text {, } \\
\mathbf{2}, 27\end{array}$ \\
\hline $\begin{array}{l}3188.522 \\
3188.10\end{array}$ & $\begin{array}{r}20 \\
3\end{array}$ & $\begin{array}{l}300 R \\
30\end{array}$ & V $E$ & $\begin{array}{l}31353.46 \\
31357.61\end{array}$ & $\begin{array}{l}a^{3} \mathrm{~F}_{3}-z^{3} \mathrm{~F}^{3} \\
a^{1} \mathrm{G}_{4}-y^{3} \mathrm{~F}_{4}^{3}\end{array}$ & $\begin{array}{l}\text { (0) } 1.01 \\
(0.67) ? n w_{1}\end{array}$ & $\begin{array}{l}(0.05) 1.03 b \\
(0.77) 1.06 b\end{array}$ \\
\hline
\end{tabular}

See footnotes at end of table. 
TABLE 1.-Second spectrum of Vanadium-Continued

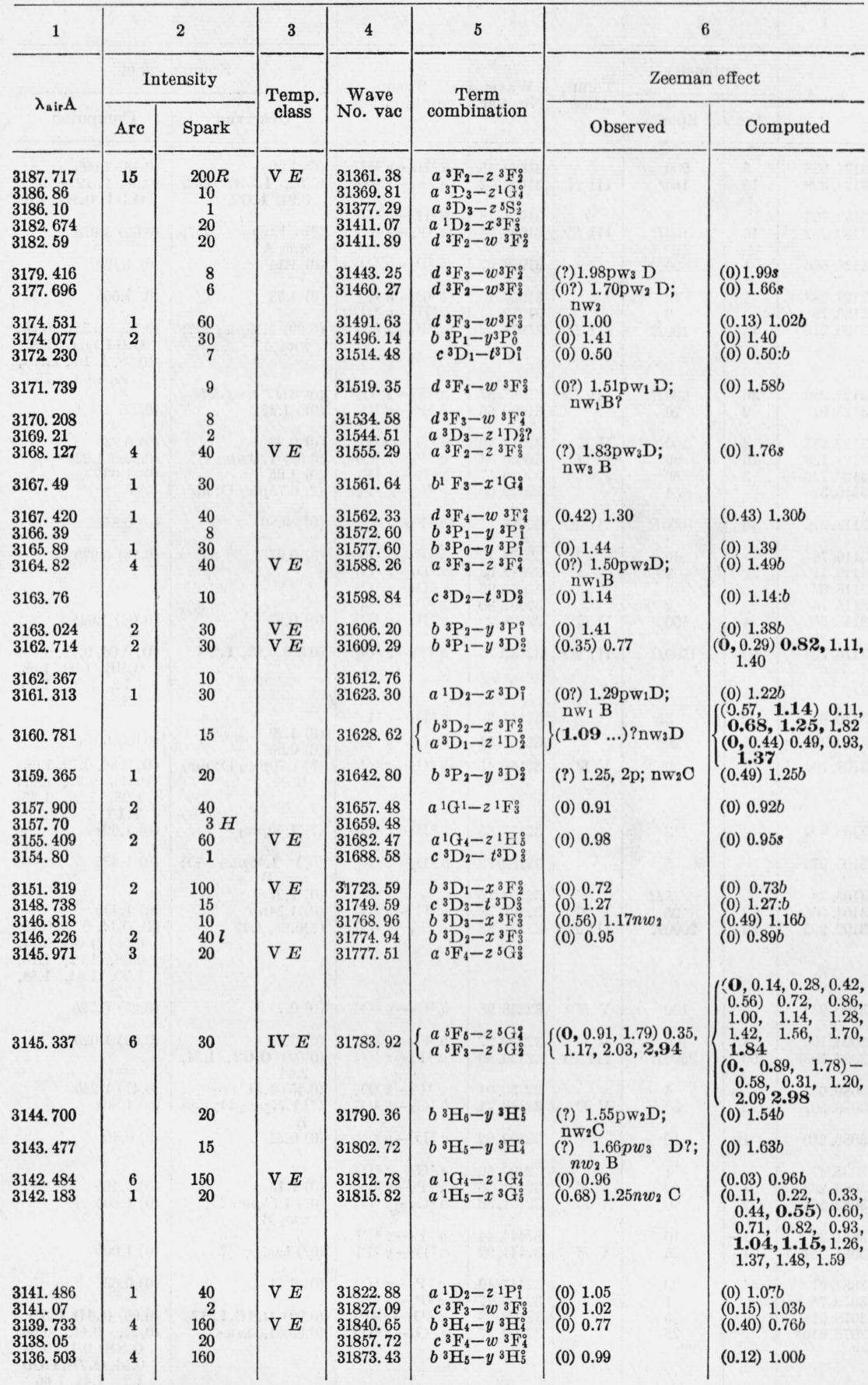

See footnotes at end of table. 
TABLE 1.-Second spectrum of Vanadium-Continued

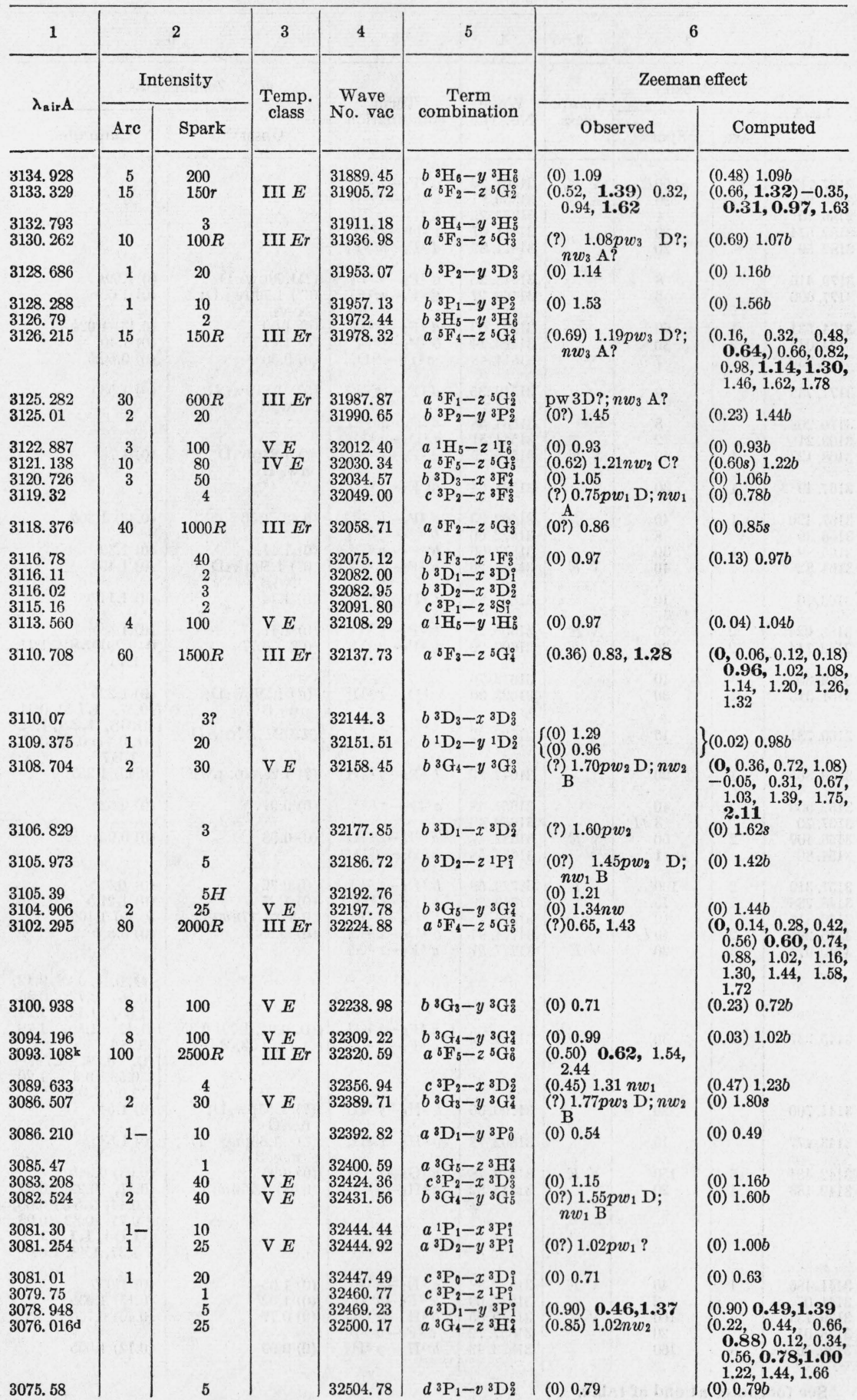

See footnotes at end of table. 
TABLE 1.-Second spectrum of Vanadium-Continued

\begin{tabular}{|c|c|c|c|c|c|c|c|}
\hline 1 & & 2 & 3 & 4 & 5 & \multicolumn{2}{|c|}{6} \\
\hline \multirow{2}{*}{$\lambda_{\mathrm{air}} \mathrm{A}$} & \multicolumn{2}{|c|}{ Intensity } & \multirow{2}{*}{$\begin{array}{l}\text { Temp. } \\
\text { class }\end{array}$} & \multirow{2}{*}{$\begin{array}{l}\text { Wave } \\
\text { No. vac }\end{array}$} & \multirow{2}{*}{$\begin{array}{c}\text { Term } \\
\text { combination }\end{array}$} & \multicolumn{2}{|c|}{ Zeeman effect } \\
\hline & Arc & Spark & & & & Observed & Computed \\
\hline $\begin{array}{l}3075.474 \\
3075.043 \\
3074.66 \\
3071.77 \\
3070.12\end{array}$ & & $\begin{array}{c}2 \\
3 \\
12 \\
2 h \\
25 l\end{array}$ & & $\begin{array}{l}32505.90 \\
32510.45 \\
32514.50 \\
32545.09 \\
32562.58\end{array}$ & $\begin{array}{l}a^{3} \mathrm{D}_{1}-y^{3} \mathrm{D}_{2}^{\circ} \\
d^{3} \mathrm{P}_{0}-v{ }^{3} \mathrm{D}_{1}^{\circ} \\
c^{3} \mathrm{P}_{1}-x{ }^{3} \mathrm{D}_{1}^{\circ} \\
c^{3} \mathrm{D}_{3}-u^{3} \mathrm{~F}_{4}^{0} \\
d^{3} \mathrm{P}_{2}-v^{3} \mathrm{D}_{3}^{0}\end{array}$ & $\begin{array}{l}\text { (0) } 0.46 \\
(0.71) \mathbf{0 . 5 9}, \mathbf{1 . 3 6} \\
\text { (0) } 1.07 n w_{1}\end{array}$ & $\begin{array}{l}\text { (0) } 0.46: \\
(0.72) \mathbf{0 . 6 3}, \mathbf{1 . 3 5} \\
\text { (0) } 1.06 b\end{array}$ \\
\hline $\begin{array}{l}3067.104 \\
3066.80 \\
3065.61 \\
3063.247 \\
3062.702\end{array}$ & $\begin{array}{r}10 \\
1 \\
8 \\
2\end{array}$ & $\begin{array}{r}200 \\
4 \\
50 \\
200 \\
20\end{array}$ & $\begin{array}{l}\text { IV } E \\
\text { V } E \\
\text { V } E\end{array}$ & $\begin{array}{l}32594.60 \\
32597.83 \\
32610.49 \\
32635.64 \\
32641.45\end{array}$ & $\begin{array}{l}a^{3} \mathrm{G}_{3}-z^{3} \mathrm{H}_{4}^{\circ} \\
b^{3} \mathrm{H}_{5}-z^{3} \mathrm{I}_{5}^{\circ} \\
c^{3} \mathrm{P}_{1}-x^{3} \mathrm{D}_{2}^{\circ} \\
b^{3} \mathrm{H}_{4}-z^{3} \mathrm{I}_{5}^{\circ} \\
a^{3} \mathrm{G}_{5}-z^{3} \mathrm{H}_{5}^{\circ}\end{array}$ & $\begin{array}{l}\text { (0) } 0.83 \\
\text { (0) } 0.96 \\
\text { (0) } 0.87 \\
(0.67) 0.99 \mathrm{nw}_{2} \mathrm{C}\end{array}$ & $\begin{array}{l}\text { (0) } 0.84 b \\
\text { (0) } 0.98 b \\
(0) 0.88 b \\
(0.65) 1.09 b\end{array}$ \\
\hline $\begin{array}{l}3062.178 \\
3057.08 \\
3055.942 \\
3054.24 \\
3053.894\end{array}$ & 3 & $\begin{array}{c}3 \\
2 \\
7 \\
7 h \\
80^{\circ}\end{array}$ & V $E$ & $\begin{array}{l}32647.03 \\
32701.47 \\
32713.65 \\
32731.88 \\
32735.59\end{array}$ & $\begin{array}{c}c^{3} \mathrm{P}_{0}-z^{1} \mathrm{P}_{1}^{\circ} \\
a^{1} \mathrm{~S}_{0}-x{ }^{3} \mathrm{D}_{1}^{\circ} \\
b^{3} \mathrm{H}_{6}-z^{3} \mathrm{I}_{6}^{\circ} \\
a^{3} \mathrm{D}_{3}-y^{3} \mathrm{D}_{3}^{\circ} \\
b^{3} \mathrm{G}_{5}-y^{3} \mathrm{~F}_{4}^{\circ}\end{array}$ & $\begin{array}{l}\text { (0) } 0.86 \\
\text { (0) } 0.61 \\
(0.76) 1.05 n w_{2} \mathrm{C} \\
\text { (0) } 1.22 n w_{1} \mathrm{~A} \\
\text { (0) } 1.12\end{array}$ & $\begin{array}{l}\text { (0) } 0.92 \\
(0) 0.63 \\
(0.78) 1.06 b \\
(0.08) 1.28 b \\
(0) 1.12 b\end{array}$ \\
\hline $\begin{array}{l}3053.39 \\
3051.308 \\
3050.735 \\
3048.891 \\
3048.65\end{array}$ & 3 & $\begin{array}{r}200 \\
3 \\
15 \\
70 \\
4\end{array}$ & V $E$ & $\begin{array}{l}32740.99 \\
32763.33 \\
32769.48 \\
32789.30 \\
32791.89\end{array}$ & $\begin{array}{l}a^{3} \mathrm{G}_{4}-z^{3} \mathrm{H}_{5}^{\circ} \\
d^{3} \mathrm{P}_{2}-v^{3} \mathrm{D}_{2}^{\circ} \\
a^{3} \mathrm{D}_{3}-y^{3} \mathrm{P}_{2}^{0} \\
b^{3} \mathrm{G}_{4}-y{ }^{3} \mathrm{~F}_{3}^{0} \\
a^{3} \mathrm{D}_{2}-y{ }^{3} \mathrm{D}_{3}^{\circ}\end{array}$ & $\begin{array}{l}\text { (0) } 0.93 \\
\text { (0) } 1.13 \\
\text { (0) } 1.02\end{array}$ & $\begin{array}{l}\text { (0) } 1.01 b \\
\text { (0) } 1.09 b \\
\text { (0) } 1.09 b\end{array}$ \\
\hline $\begin{array}{l}3048.214 \\
3043.54\end{array}$ & $\begin{array}{l}6 \\
?\end{array}$ & $\begin{array}{l}200 \\
40+I\end{array}$ & V E & $\begin{array}{l}32796.59 \\
32846.95\end{array}$ & $\begin{array}{l}b^{3} \mathrm{H}_{5}-z^{3} \mathrm{I}_{6}^{\circ} \\
b^{3} \mathrm{G}_{4}-y^{3} \mathrm{~F}_{4}^{\circ}\end{array}$ & $\begin{array}{l}\text { (0) } 0.96 \\
(0.54) 1.14 \quad p w_{2} \mathrm{~B} ; \\
n w_{2} \mathrm{C}\end{array}$ & $\begin{array}{l}\text { (0) } 0.90 b \\
(0.50) 1.11 b\end{array}$ \\
\hline $\begin{array}{l}3042.27 \\
3041.42 \\
3039.767\end{array}$ & $\begin{array}{l}3 \\
2\end{array}$ & $\begin{array}{r}80 \\
60 \\
2\end{array}$ & $\begin{array}{l}\text { IV } E \\
\text { V } E\end{array}$ & $\begin{array}{l}32860.66 \\
32869.84 \\
32887.72\end{array}$ & $\begin{array}{l}b^{3} \mathrm{G}_{3}-y{ }^{3} \mathrm{~F}_{2}^{0} \\
b^{3} \mathrm{G}_{3}-y{ }^{3} \mathrm{~F}_{3}^{0} \\
a^{1} \mathrm{D}_{2}-w^{3} \mathrm{D}_{2}\end{array}$ & $\begin{array}{l}\text { (0) } 0.77 \\
\text { (?) } 0.93,2 p ; n w_{2} \mathrm{C} \\
\text { (0) } 1.13\end{array}$ & $\begin{array}{l}\text { (0) } 0.89 b \\
(0.59) 0.88 b \\
(0.14) 1.06 b\end{array}$ \\
\hline $\begin{array}{l}3038.520 \\
3038.00 \\
3036.07 \\
3035.14 \\
3034.41\end{array}$ & 1 & $\begin{array}{l}30 \\
2 H ? \\
2 \\
3 H \\
4 H\end{array}$ & & $\begin{array}{l}32901.21 \\
32906.84 \\
32927.76 \\
32937.85 \\
32945.78\end{array}$ & $\begin{array}{l}a{ }^{1} \mathrm{~S}_{0}-z{ }^{1} \mathrm{P}_{1}^{\circ} \\
z^{3} \mathrm{~F}_{2}^{\circ}-e^{3} \mathrm{P}_{3} \\
b^{3} \mathrm{G}_{3}-y^{3} \mathrm{~F}_{4}^{\circ} \\
z^{3} \mathrm{G}_{5}^{\circ}-e^{5} \mathrm{H}_{4}\end{array}$ & (0) 0.87 & (0) 0.92 \\
\hline $\begin{array}{l}3033.821 \\
3033.445 \\
3032.187 \\
3029.56 \\
3028.042\end{array}$ & $\begin{array}{l}8 \\
6\end{array}$ & $\begin{array}{r}300 \\
200 \\
3 \\
7 \\
50\end{array}$ & $\begin{array}{l}\mathrm{V} E \\
\mathrm{~V} E\end{array}$ & $\begin{array}{l}32952.17 \\
32956.25 \\
32969.93 \\
32998.52 \\
33015.05\end{array}$ & $\begin{array}{l}a^{3} \mathrm{G}_{5}-z{ }^{3} \mathrm{H}_{6}^{\circ} \\
b^{3} \mathrm{H}_{6}-z{ }^{3} \mathrm{I}_{7}^{0} \\
b{ }^{1} \mathrm{G}_{4}-y{ }^{3} \mathrm{H}_{4}^{0} \\
a^{5} \mathrm{P}_{3}-z{ }^{3} \mathrm{P}_{2}^{0} \\
b^{3} \mathrm{P}_{1}-z{ }^{3} \mathrm{~S}_{1}^{0}\end{array}$ & $\begin{array}{l}\text { (0) } 0.98 \\
\text { (0) } 1.02 \\
(0) 1.72 \\
(0.49) \mathbf{1 . 3 5}, \mathbf{2 . 1 0}\end{array}$ & $\begin{array}{l}\text { (0) } 1.03 b \\
\text { (0) } 1.02 b \\
\text { (0) } 1.76 b \\
(0.45) \mathbf{1 . 4 0 , 1 . 8 5}\end{array}$ \\
\hline $\begin{array}{l}3027.600 \\
3025.68 \\
3024.981\end{array}$ & $\begin{array}{l}1- \\
1\end{array}$ & $\begin{array}{r}15 \\
1 \\
50\end{array}$ & & $\begin{array}{l}33019.88 \\
33040.83 \\
33048.46\end{array}$ & $\begin{array}{l}b^{3} \mathrm{P}_{0}-z{ }^{3} \mathrm{Si} \\
b{ }^{1} \mathrm{G}_{4}-y^{3} \mathrm{H}_{5}^{\circ} \\
b^{3} \mathrm{P}_{2}-z^{3} \mathrm{~S}_{i}^{\circ}\end{array}$ & $\begin{array}{l}\text { (0) } 1.85 \\
\text { (?) } \mathbf{0 . 9 4}, 1.39,1.89\end{array}$ & $\begin{array}{l}\text { (0) } 1.85 \\
(\mathbf{0 , 0 . 4 7 )} \mathbf{0 . 9 1 , 1 . 3 8}\end{array}$ \\
\hline $\begin{array}{l}3023.882 \\
3022.57\end{array}$ & $\begin{array}{l}1 \\
2\end{array}$ & $\begin{array}{l}20 \\
40\end{array}$ & & $\begin{array}{l}33060.47 \\
33074.82\end{array}$ & $\begin{array}{l}b^{3} \mathrm{G}_{5}-z{ }^{1} \mathrm{H}_{5}^{\circ} \\
a^{5} \mathrm{P}_{1}-z^{3} \mathrm{P}_{0}^{0}\end{array}$ & $\begin{array}{l}(0.82) 1.00 \mathrm{nw}_{2} \\
(0) 2.35\end{array}$ & $\begin{array}{l}(0.86) 1.06 b \\
\text { (0) } 2.39\end{array}$ \\
\hline $\begin{array}{l}3022.146 \\
3020.65 \\
3020.25 \\
3019.09 \\
3018.96\end{array}$ & & $\begin{array}{l}4 \\
6 \\
7 H l \\
3 \\
3 h\end{array}$ & & $\begin{array}{l}33079.46 \\
33095.85 \\
33100.23 \\
3311295 \\
33114.37\end{array}$ & $\begin{array}{l}b^{3} \mathrm{P}_{1}-x^{3} \mathrm{~F}_{2}^{\circ} \\
a^{5} \mathrm{P}_{2}-z^{3} \mathrm{P}_{1}^{0} \\
b^{3} \mathrm{P}_{2}-x^{3} \mathrm{~F}_{2}^{0}\end{array}$ & & \\
\hline $\begin{array}{l}3016.775 \\
3016.14 \\
3015.98 \\
3014.822\end{array}$ & 5 & $\begin{array}{r}120 \\
15 \\
10 \\
100\end{array}$ & $\begin{array}{l}\text { V } E \\
\text { V } E\end{array}$ & $\begin{array}{l}33138.35 \\
33145.33 \\
33147.09 \\
33159.82\end{array}$ & $\begin{array}{l}a{ }^{5} \mathrm{P}_{3}-z{ }^{5} \mathrm{P}_{2}^{\circ} \\
a^{5} \mathrm{P}_{2}-z^{3} \mathrm{P}_{2}^{0} \\
b^{3} \mathrm{G}_{4}-2{ }^{1} \mathrm{~F}_{3}^{0} \\
a^{5} \mathrm{P}_{2}-z^{5} \mathrm{P}_{1}^{0}\end{array}$ & $\begin{array}{l}\text { (?) } 1.41 \\
\text { (?) } 1.03,1.71,2.32\end{array}$ & $\begin{array}{l}\text { (0) } 1.50 s \\
(0,0.50) \mathbf{1 . 2 8}, 1.78\end{array}$ \\
\hline 3013.102 & 3 & 80 & V $E$ & 33178.75 & $a^{5} \mathrm{P}_{1}-z^{3} \mathrm{P}_{1}^{0}$ & (?) $1.43,2.35$ & $\begin{array}{c}2.28 \\
(0.95)\end{array}$ 1.44, $\mathbf{2} .39$ \\
\hline $\begin{array}{l}3012.020 \\
3011.258\end{array}$ & 1 & $\begin{array}{r}30 \\
1\end{array}$ & & $\begin{array}{l}33190.67 \\
33199.07\end{array}$ & $b^{3} \mathrm{G}_{5}-z^{1} \mathrm{G}_{4}^{\circ}$ & (?) $1.57 n w_{2} \mathrm{~B}$ & (0) $1.56 b$ \\
\hline 3008.610 i & 2 & $\begin{array}{r}1 \\
70\end{array}$ & VE & $\begin{array}{l}33199.07 \\
33228.28\end{array}$ & $a^{5} \mathrm{P}_{1}-z^{3} \mathrm{P}_{2}^{\circ}$ & (?) $\mathbf{0 . 4 9}, 1.39,2.31$ & $\begin{array}{l}(\mathbf{0}, 0.91) \mathbf{0 . 5 7}, 1.48 \\
2.39\end{array}$ \\
\hline $\begin{array}{l}3008.508 \\
3007.296\end{array}$ & 1 & $\begin{array}{l}15 \\
15\end{array}$ & & $\begin{array}{l}33229.41 \\
33242.80\end{array}$ & $\begin{array}{l}b^{3} \mathrm{D}_{1}-w^{3} \mathrm{D}_{\mathrm{i}} \\
a^{5} \mathrm{P}_{1}-z^{5} \mathrm{P}_{1}^{0}\end{array}$ & (?) 2.30 & $(0.11) 2.34 b$ \\
\hline $\begin{array}{l}3007.035 \\
3006.502 \\
3005.813 \\
3003.461 \\
3001.93\end{array}$ & 3 & $\begin{array}{r}1 \\
20 \\
30 \\
80 \\
2\end{array}$ & V $E$ & $\begin{array}{l}33245.69 \\
33251.58 \\
33259.20 \\
33285.25 \\
33302.22\end{array}$ & $\begin{array}{l}b^{3} \mathrm{D}_{3}-w{ }^{3} \mathrm{D}_{2}^{\circ} \\
b^{3} \mathrm{D}_{2}-w^{3} \mathrm{D}_{2}^{\circ} \\
b^{3} \mathrm{P}_{2}-x{ }^{3} \mathrm{~F}_{3}^{0} \\
a^{5} \mathrm{P}_{2}-z{ }^{5} \mathrm{P}_{2}^{\circ} \\
b^{3} \mathrm{G}_{4}-z{ }^{1} \mathrm{G}_{4}^{\circ}\end{array}$ & $\begin{array}{ll}\text { (?) } & 1.13 \\
\text { (?) } & 0.45 n w_{3} \mathrm{~A} \\
\text { (?) } & 1.69 \\
\text { (?) } & 0.96\end{array}$ & $\begin{array}{l}(0.27) 1.18 b \\
(0) 0.45 s \\
(0.18) 1.73 b \\
(0.23) 1.00 b\end{array}$ \\
\hline
\end{tabular}

See footnotes at end of table. 
TABLE 1.-Second spectrum of Vanadium-Continued

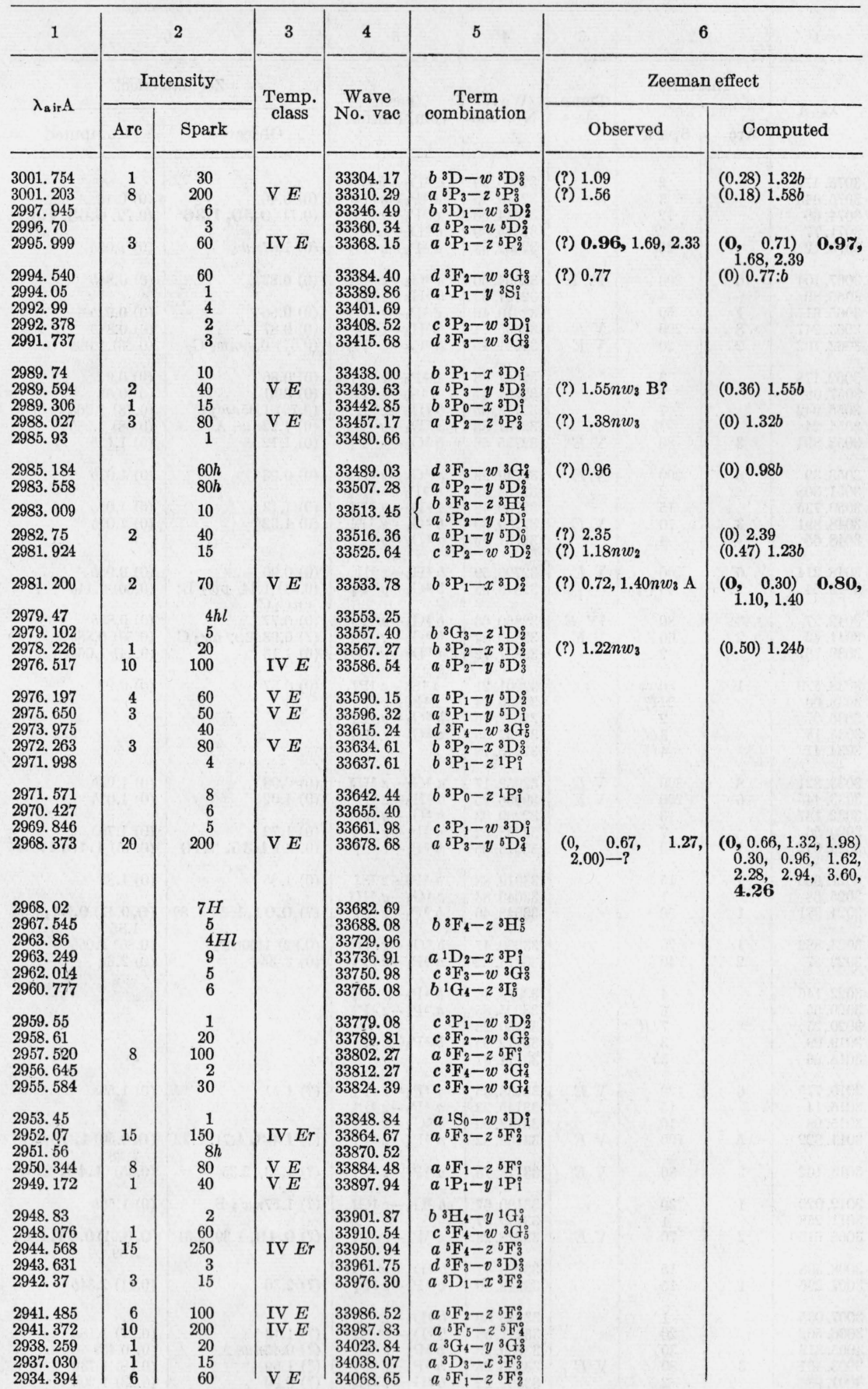

See footnotes at end of table, 
TABLE 1.-Second spectrum of Vanadium-Continued

\begin{tabular}{|c|c|c|c|c|c|c|c|}
\hline 1 & & 2 & 3 & 4 & 5 & t & 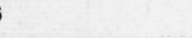 \\
\hline \multirow{2}{*}{$\lambda_{\mathrm{a} \text { ir } \mathrm{A}}$} & \multicolumn{2}{|c|}{ Intensity } & \multirow{2}{*}{$\begin{array}{c}\text { Temp. } \\
\text { class }\end{array}$} & \multirow{2}{*}{$\begin{array}{c}\text { Wave } \\
\text { No. vac }\end{array}$} & \multirow{2}{*}{$\begin{array}{c}\text { Term } \\
\text { combination }\end{array}$} & \multicolumn{2}{|c|}{ Zeeman effect } \\
\hline & Arc & Spark & & & & Observed & Computed \\
\hline $\begin{array}{l}2933.833 \\
2932.323 \\
2931.859 \\
2931.624 \\
2930.798\end{array}$ & $\begin{array}{r}1 \\
3 \\
1 \\
1 \\
10\end{array}$ & $\begin{array}{r}15 \\
60 \\
10 \\
20 \\
150\end{array}$ & $\begin{array}{l}\text { V } \frac{E}{\text { V }} \\
\text { IV } E r\end{array}$ & $\begin{array}{l}34075.16 \\
34092.71 \\
34098.10 \\
34100.84 \\
34110.45\end{array}$ & $\begin{array}{l}a^{3} \mathrm{G}_{5}-y{ }^{3} \mathrm{G}_{4}^{\circ} \\
b^{3} \mathrm{D}_{3}-x{ }^{3} \mathrm{P}_{2}^{0} \\
a^{3} \mathrm{D}_{2}-x{ }^{3} \mathrm{~F}_{3}^{0} \\
b^{3} \mathrm{D}_{2}-x x^{3} \mathrm{P}_{1}^{1} \\
a^{5} \mathrm{~F}_{3}-z^{5} \mathrm{~F}_{3}^{0}\end{array}$ & & \\
\hline $\begin{array}{l}2930.132 \\
2929.017 \\
2926.442 \\
2926.35 \\
2925.51\end{array}$ & 2 & $\begin{array}{c}25 \\
4 \\
40 \\
10 h \\
3 h\end{array}$ & V $E$ & $\begin{array}{l}34118.20 \\
34131.19 \\
34161.23 \\
34162.29 \\
34172.10\end{array}$ & $\begin{array}{l}a^{3} \mathrm{G}_{3}-y{ }^{3} \mathrm{G}_{3}^{0} \\
d^{3} \mathrm{~F}_{2}-v \\
a^{1} \mathrm{D}_{2}^{3} \mathrm{D}_{2}-y \\
{ }^{1} \mathrm{~F}_{3}^{3} \\
d^{3} \mathrm{~B}_{3}-v \\
a^{3} \mathrm{D}_{2}^{2} \\
{ }^{1} \mathrm{G}_{4}-y^{3} \mathrm{H}_{4}^{0}\end{array}$ & & \\
\hline $\begin{array}{l}2925.288 \\
2924.633 \\
2924.017 \\
2923.340\end{array}$ & $\begin{array}{r}1 \\
30 \\
40 \\
1\end{array}$ & $\begin{array}{c}15 \\
250 R \\
300 R \\
20\end{array}$ & $\begin{array}{l}\text { V } E \\
\text { IV } E r \\
\text { IV } E r\end{array}$ & $\begin{array}{l}34174.69 \\
34182.35 \\
34189.55 \\
34197.46\end{array}$ & $\begin{array}{l}a^{3} \mathrm{G}_{4}-y^{3} \mathrm{G}_{4}^{\circ} \\
a^{5} \mathrm{~F}_{4}-z^{5} \mathrm{~F}_{4}^{4} \\
a^{5} \mathrm{~F}_{5}-z^{5} \mathrm{~F}_{5}^{5} \\
a^{3} \mathrm{G}_{5}-y^{3} \mathrm{G}_{5}^{0}\end{array}$ & & \\
\hline 2920.377 & 10 & 100 & IV $E$ & 34232.16 & $\left\{\begin{array}{l}a^{5} \mathrm{~F}_{2}-z^{5} \mathrm{~F}_{3}^{0} \\
a^{5} \mathrm{~F}_{3}-z^{3} \mathrm{D}_{2}^{8}\end{array}\right.$ & & \\
\hline $\begin{array}{l}2919.989 \\
2919.681 \\
2918.21 \\
2917.365 \\
2917.230\end{array}$ & $\begin{array}{l}4 \\
1\end{array}$ & $\begin{array}{c}50 \\
6 h \\
15 h \\
50 \\
7\end{array}$ & V $E$ & $\begin{array}{l}34236.71 \\
34240.32 \\
34257.58 \\
34267.50 \\
34269.09\end{array}$ & $\begin{array}{l}a^{5} \mathrm{~F}_{4}-z{ }^{3} \mathrm{D}_{3}^{\circ} \\
d^{3} \mathrm{~F}_{2}-n^{3} \mathrm{D}_{1}^{\circ} \\
a^{5} \mathrm{~F}_{2}-z{ }^{3} \mathrm{D}_{1}^{\circ} \\
a^{3} \mathrm{G}_{3}-y^{3} \mathrm{G}_{4}\end{array}$ & & \\
\hline $\begin{array}{l}2915.875 \\
2915.330 \\
2914.87 \\
2914.298 \\
2913.716\end{array}$ & $\begin{array}{l}5 \\
? \\
2\end{array}$ & $\begin{array}{l}40 \\
30 \\
10+\mathrm{I} \\
40 \\
2\end{array}$ & & $\begin{array}{l}34285.01 \\
34291.42 \\
34296.83 \\
34303.56 \\
34310.41\end{array}$ & $\begin{array}{l}c^{3} \mathrm{~F}_{4}-v^{3} \mathrm{D}_{3}^{\circ} \\
b^{3} \mathrm{D}_{1}-x^{3} \mathrm{P}_{0}^{0} \\
a^{3} \mathrm{G}_{4}-y^{3} \mathrm{G}_{5}^{\circ} \\
a^{3} \mathrm{D}_{3}-x^{3} \mathrm{~F}_{4}^{4} \\
a^{3} \mathrm{D}_{2}-x^{3} \mathrm{D}_{1}^{\circ}\end{array}$ & & \\
\hline $\begin{array}{l}2913.04 \\
2912.50 \\
2911.654 \\
2911.050 \\
2910.380\end{array}$ & $\begin{array}{l}15 \\
15\end{array}$ & $\begin{array}{c}3 \\
10 H l \\
7 \\
160 r \\
150 r\end{array}$ & & $\begin{array}{l}34318.38 \\
34324.74 \\
34334.71 \\
34341.83 \\
34349.74\end{array}$ & $\begin{array}{l}a^{3} \mathrm{D}_{1}-x^{3} \mathrm{D}^{\circ} \\
a^{5} \mathrm{~b}_{3}-z^{3} \mathrm{~F}_{i}^{\circ} \\
a^{5} \mathrm{~s}_{1}-z^{3} \mathrm{D}_{\mathrm{i}}^{\circ}\end{array}$ & & \\
\hline $\begin{array}{l}2910.007 \\
2908.810 \\
2908.44 \\
2907.457 l \\
2906.448\end{array}$ & $\begin{array}{r}15 \\
30 \\
2 \\
10 \\
15\end{array}$ & $\begin{array}{l}140 r \\
300 R \\
20 \\
120 \\
150 r\end{array}$ & $\begin{array}{l}\text { IV } E r \\
\text { IV } E r \\
\text { IVE } \\
\text { IVEr }\end{array}$ & $\begin{array}{l}34354.14 \\
34368.28 \\
34372.65 \\
34384.27 \\
34396.21\end{array}$ & $\begin{array}{l}a^{5} \mathrm{~S}_{2}-z^{3} \mathrm{D}_{2}^{2} \\
a^{5} \mathrm{~F}_{5}-z^{5} \mathrm{D}_{4}^{4} \\
c^{3} \mathrm{P}_{2}-x^{3} \mathrm{P}_{2}^{2} \\
a^{5} \mathrm{~F}_{4}-z z^{5} \mathrm{~F}_{5}^{5} \\
a^{5} \mathrm{~F}_{3}-z^{3} \mathrm{D}_{3}^{3}\end{array}$ & $\begin{array}{l}(1.00) 0.68 \\
\text { (?) } 2.53 p w_{3} \mathrm{D} ; n w_{2}\end{array}$ & $\begin{array}{l}(0) 0.64 s \\
(0) 1.60 b\end{array}$ \\
\hline $\begin{array}{l}2905.609 \\
2905.307 \\
2904.985 \\
2903.548 \\
2903.068\end{array}$ & 10 & $\begin{array}{r}15 \\
1 \\
15 \\
3 \\
100\end{array}$ & V $E$ & $\begin{array}{l}34406.14 \\
24409.72 \\
34413.53 \\
34430.56 \\
34436.25\end{array}$ & $\begin{array}{l}a^{3} \mathrm{D}_{2}-x{ }^{3} \mathrm{D}_{2}^{\circ} \\
a^{3} \mathrm{D}_{3}-x \\
a^{3} \mathrm{D}_{3}^{2} \\
a^{3} \mathrm{D}_{1}-x \\
{ }^{3} \mathrm{~F}_{1}-z^{3} \mathrm{D}_{2}^{2}\end{array}$ & & \\
\hline $\begin{array}{l}2902.54 \\
2899.936 \\
2897.899 \\
2896.870 \\
2896.198 \text { i }\end{array}$ & 10 & $\begin{array}{r}1 \\
4 \\
20 \\
2 \\
100\end{array}$ & V $E$ & $\begin{array}{l}34442.52 \\
34473.44 \\
34497.67 \\
34509.93 \\
34517.93\end{array}$ & $\begin{array}{c}a^{3} \mathrm{D}_{2}-x^{3} \mathrm{D}_{3}^{0} \\
c^{3} \mathrm{~F}_{3}-v^{3} \mathrm{D}_{2}^{\circ} \\
a^{3} \mathrm{D}_{2}-z{ }^{1} \mathrm{P}_{1}^{0} \\
a^{5} \mathrm{~F}_{2}-z \\
{ }^{3} \mathrm{D}_{3}^{3}\end{array}$ & $(0 ?) ? p w_{2} \mathrm{D} ; n w_{1}$ & (0) $1.67 b$ \\
\hline $\begin{array}{l}2895.609 \\
2894.833 \\
2893.314 \\
2892.650 \\
2892.434\end{array}$ & $\begin{array}{l}20 \\
15 \\
10\end{array}$ & $\begin{array}{c}4 \\
3 \\
250 \\
200 r \\
150 r\end{array}$ & $\begin{array}{l}\text { IV } E r \\
\text { IV } E r \\
\text { V } E r\end{array}$ & $\begin{array}{l}34524.96 \\
34534.21 \\
34552.34 \\
34560.27 \\
34562.85\end{array}$ & $\begin{array}{l}b^{3} \mathrm{D}_{2}-y^{1} \mathrm{~F}_{3}^{\circ} \\
a^{3} \mathrm{~F}_{1}-v \\
a^{5} \mathrm{P}_{1} \\
a^{5} \mathrm{~F}_{4}-z \\
a^{5} \mathrm{~S}_{3} \mathrm{D}_{3}^{\circ}-z \\
a^{5} \mathrm{D}_{2}^{\circ} \\
a^{5} \mathrm{~F}_{4}-z^{5} \mathrm{D}_{4}^{\circ}\end{array}$ & & \\
\hline 2891.636 & 10 & $150 r$ & IV $E r$ & 34572.39 & $a^{5} \mathrm{~F}_{2}-z^{5} \mathrm{Di}_{\mathrm{i}}$ & $(0,0.69) ?$ & $\begin{array}{l}(0,0.42) 0.55,0.97 \\
1.39\end{array}$ \\
\hline $\begin{array}{l}2890.553 \\
2890.144 \\
2889.614 \\
2888.244\end{array}$ & $\begin{array}{r}1 \\
15 \\
5\end{array}$ & $\begin{array}{r}5 \\
7 \\
100 \\
80\end{array}$ & $\begin{array}{l}\mathrm{V} \\
\mathrm{V} \\
E\end{array}$ & $\begin{array}{l}34585.34 \\
34590.24 \\
34596.58 \\
34612.99\end{array}$ & $\begin{array}{l}b^{3} \mathrm{P}_{1}-w^{3} \mathrm{D}_{1}^{\circ} \\
b^{3} \mathrm{P}_{0}-w^{3} \mathrm{D}_{0}^{1} \\
a^{5} \mathrm{~F}_{1}-z^{5} \mathrm{D}_{0}^{0} \\
a^{3} \mathrm{G}_{8}-y^{3} \mathrm{~F}_{4}^{0}\end{array}$ & & \\
\hline $\begin{array}{l}2887.75 \\
2887.158 \\
2886.967\end{array}$ & 1 & $\begin{array}{r}1 \\
8 \\
10\end{array}$ & & $\begin{array}{l}34618.91 \\
34626.01 \\
34628.30\end{array}$ & $\begin{array}{l}b^{3} \mathrm{P}_{2}-v:{ }^{3} \mathrm{D}_{1}^{\circ} \\
c^{3} \mathrm{P}_{1}-x^{3} \mathrm{P}_{2}^{0} \\
c^{3} \mathrm{P}_{1}-x^{3} \mathrm{P}_{1}^{\circ}\end{array}$ & & \\
\hline $\begin{array}{l}2884.776 \\
2884.064\end{array}$ & 20 & $\begin{array}{r}150 \\
6\end{array}$ & V $E$ & $\begin{array}{l}34654.60 \\
34663.15\end{array}$ & $\left\{\begin{array}{r}a^{5} \mathrm{~F}_{1}-z^{5} \mathrm{D}_{1}^{\mathrm{i}} \\
a^{3} \mathrm{G}_{4}-y^{3} \mathrm{~F}_{3}^{3} \\
c^{3} \mathrm{~F}_{2}-v^{3} \mathrm{D}_{1}^{\mathrm{s}}\end{array}\right.$ & \}$(0) 0.64$ & $\{\overline{(0)} 1.02 b$ \\
\hline
\end{tabular}

See footnotes at end of table. 
TABLE 1.-Second spectrum of Vanadium-Continued

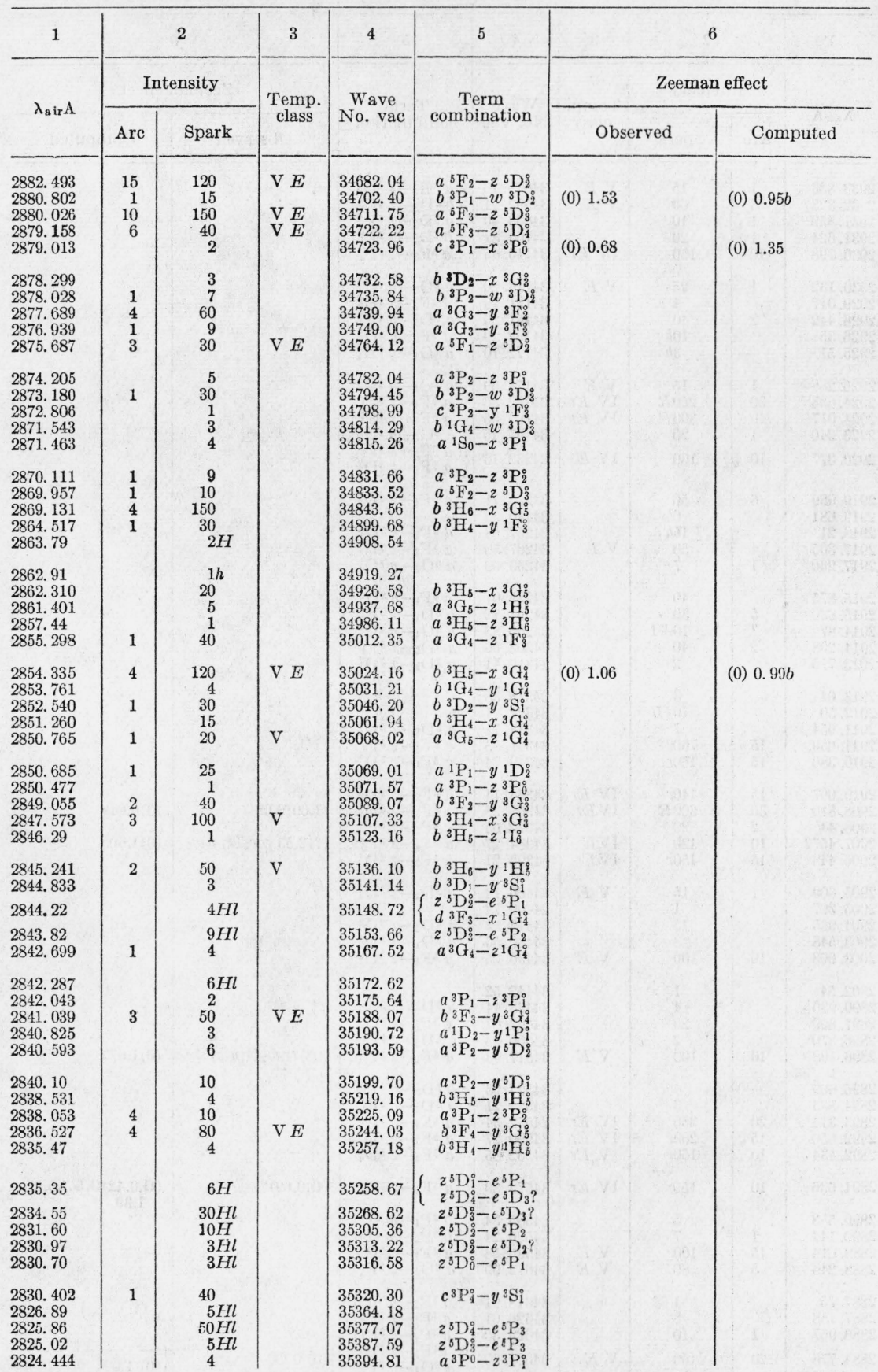

See footnotes at end of table. 
TABLE 1.-Second spectrum of Vanadium-Continued

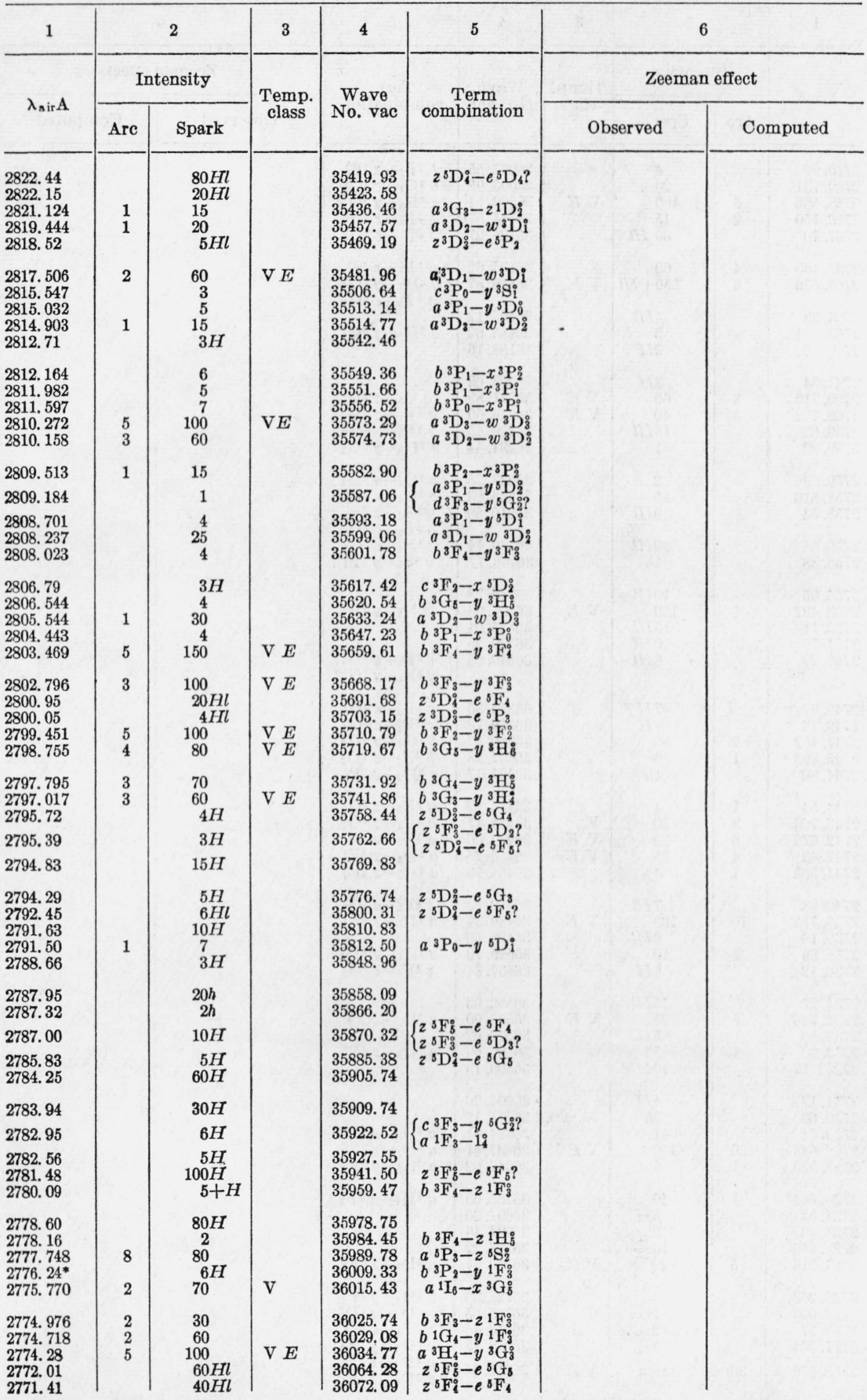

See footnotes at end of table. 
TABLE 1.-Second spectrum of Vanadium-Continued

\begin{tabular}{|c|c|c|c|c|c|c|c|}
\hline 1 & & 2 & 3 & 4 & 5 & & \\
\hline \multirow{2}{*}{$\lambda_{\mathrm{sir}} \mathrm{A}$} & \multicolumn{2}{|c|}{ Intensity } & \multirow{2}{*}{$\begin{array}{l}\text { Temp. } \\
\text { class }\end{array}$} & \multirow{2}{*}{$\begin{array}{c}\text { Wave } \\
\text { No. vac }\end{array}$} & \multirow{2}{*}{$\begin{array}{c}\text { Term } \\
\text { combination }\end{array}$} & \multicolumn{2}{|c|}{ Zeeman effect } \\
\hline & Arc & Spark & & & & Observed & Computed \\
\hline $\begin{array}{l}2770.99 \\
2769.731 \\
2768.566 \\
2768.150 \\
2767.10\end{array}$ & $\begin{array}{l}5 \\
2\end{array}$ & $\begin{array}{c}4 \\
20 \\
100 \\
15 \\
30 \mathrm{Hl}\end{array}$ & V $E$ & $\begin{array}{l}36077.55 \\
36093.95 \\
36109.14 \\
36114.57 \\
36128.27\end{array}$ & 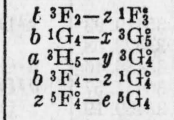 & & \\
\hline $\begin{array}{l}2766.460 \\
2765.676\end{array}$ & $\begin{array}{l}4 \\
6\end{array}$ & $\begin{array}{l}60 \\
150+H l\end{array}$ & V $E$ & $\begin{array}{l}36136.62 \\
36146.87\end{array}$ & $\left\{\begin{array}{c}a^{3} \mathrm{P}_{2}-z^{5} \mathrm{~S}_{2} \\
a^{3} \mathrm{H}_{6}-y^{3} \mathrm{G}_{5}^{8} \\
z{ }^{5} \mathrm{~F}^{0}-e^{6} \mathrm{G}_{3}\end{array}\right.$ & & \\
\hline $\begin{array}{l}2764.28 \\
2762.714 \\
2762.52\end{array}$ & & $\begin{array}{l}4 H l \\
3 \\
3 H\end{array}$ & & $\begin{array}{l}36165.12 \\
36185.62 \\
36188.16\end{array}$ & $a^{3} \mathrm{H}_{4}-y^{3} \mathrm{G}_{4}$ & & \\
\hline $\begin{array}{l}2761.34 \\
2770.710 \\
2760.122 \\
2759.60 \\
2759.22\end{array}$ & $\begin{array}{l}3 \\
3\end{array}$ & $\begin{array}{l}3 H \\
60 \\
40 \\
15 H l \\
1\end{array}$ & $\begin{array}{l}\mathrm{V} \\
\mathrm{V} \\
E\end{array}$ & $\begin{array}{l}36203.63 \\
36211.89 \\
36219.60 \\
36226.45 \\
36231.44\end{array}$ & $\begin{array}{c}a{ }^{1} \mathrm{I}_{6}-2{ }^{1} \mathrm{I}_{6}^{\circ} \\
a^{5} \mathrm{P}_{1}-z{ }^{3} \mathrm{~S}_{2}^{\circ} \\
z{ }^{3} \mathrm{~F}_{3}^{\circ}-e^{5} \mathrm{G}_{3} \\
a^{3} \mathrm{H}_{5}-y^{3} \mathrm{G}_{5}^{\circ}\end{array}$ & & \\
\hline $\begin{array}{l}2759.08 \\
2758.810 \\
2758.53\end{array}$ & & $\begin{array}{l}\stackrel{2}{15} \\
9 H l\end{array}$ & & $\begin{array}{l}36233.28 \\
36236.83 \\
36240.50\end{array}$ & 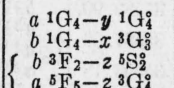 & & \\
\hline $\begin{array}{l}2756.58 \\
2756.38\end{array}$ & & $\underset{4 h}{20 H l}$ & & $\begin{array}{l}36266.14 \\
36268.77\end{array}$ & $\begin{array}{r}z^{5} \mathrm{~F}_{4}^{0}-e^{5} \mathrm{G}_{6} \\
a^{1} \mathrm{~S}_{0}-y^{1} \mathrm{P}_{1}^{1}\end{array}$ & & \\
\hline $\begin{array}{l}2755.05 \\
2753.407 \\
2752.11 \\
2751.79 \\
2750.29\end{array}$ & 7 & $\begin{array}{c}10 H l \\
150 \\
15 H l \\
10 H l \\
8 H l\end{array}$ & V $E$ & $\begin{array}{l}36286.28 \\
36307.93 \\
35325.04 \\
36329.26 \\
36349.08\end{array}$ & $\left\{\begin{array}{c}a^{1} \mathrm{I}_{6}-y^{1} \mathrm{H}_{5}^{\circ} \\
\left\{\begin{array}{l}c^{3} \mathrm{~F}_{4}-y^{5} \mathrm{G}_{3}^{\circ} ? \\
d^{3} \mathrm{~F}_{4}-y^{6} \mathrm{G}_{4}^{2} ?\end{array}\right.\end{array}\right.$ & & \\
\hline $\begin{array}{l}2749.97 \\
2749.48 \\
2747.462 \\
2745.893 \\
2744.80\end{array}$ & $\begin{array}{l}2 \\
1\end{array}$ & $\begin{array}{c}7 H \\
8 H \\
80 \\
6 \\
1 h\end{array}$ & & $\begin{array}{l}36353.30 \\
36359.78 \\
36386.49 \\
36407.28 \\
36421.77\end{array}$ & $\begin{array}{c}z^{5} \mathrm{~F}_{2}^{0}-e^{5} \mathrm{G}_{2} \\
2^{5} \mathrm{~F}_{3}^{0}-e^{5} \mathrm{G}_{4} \\
b^{1} \mathrm{G}_{4}-y{ }^{1} \mathrm{H}_{5}^{0} \\
b^{3} \mathrm{~F}_{2}-z 1^{1} \mathrm{D}_{2}^{2} \\
a^{3} \mathrm{D}_{2}-x^{3} \mathrm{P}_{2}^{2}\end{array}$ & & \\
\hline $\begin{array}{l}2744.54 \\
2743.768 \\
2742.670 \\
2742.43 \\
2741.563\end{array}$ & $\begin{array}{l}1 \\
3 \\
6 \\
4 \\
1\end{array}$ & $\begin{array}{r}4 \\
20 \\
30 \\
25 \\
4\end{array}$ & $\begin{array}{l}\mathrm{V} \\
\mathrm{V} \\
\mathrm{V} \\
E\end{array}$ & $\begin{array}{l}36425.22 \\
36435.47 \\
36450.06 \\
36453.25 \\
36464.78\end{array}$ & $\begin{array}{l}a^{5}{ }^{5} \mathrm{~F}_{3}-z{ }^{3} \mathrm{G}_{3}^{\circ} \\
a^{5} \mathrm{~F}_{4}-z^{3} \mathrm{G}_{4}^{4} \\
a^{5} \mathrm{~F}_{5}-z{ }^{3} \mathrm{G}_{5}^{5} \\
a^{5} \mathrm{D}_{1}-z{ }^{5} \mathrm{~F}_{1}^{1} \\
a^{5} \mathrm{D}_{3}-z z^{5} \mathrm{~F}_{2}^{2}\end{array}$ & & \\
\hline $\begin{array}{l}2740.98 \\
2739.715 \\
2739.18 \\
2736.69 \\
2736.12\end{array}$ & $\begin{array}{r}10 \\
1\end{array}$ & $\begin{array}{c}7 \mathrm{Hl} \\
100 \\
8 H l \\
10 \\
4 H\end{array}$ & V $E$ & \begin{tabular}{l|}
36472.53 \\
36489.37 \\
36496.50 \\
36529.70 \\
36537.31
\end{tabular} & $\begin{array}{l}2^{2}{ }^{5} \mathrm{~F}_{2}^{0}-e^{5} \mathrm{G}_{3} \\
a^{5} \mathrm{D}_{0}-z^{5} \mathrm{~F}_{1}^{1} \\
a^{3} \mathrm{G}_{4}-y^{3} \mathrm{D}_{3}^{\circ} \\
z^{5} \mathrm{~F}_{1}^{\circ}-e^{5} \mathrm{G}_{2}\end{array}$ & & \\
\hline $\begin{array}{l}2734.27 \\
2733.906 \\
2733.00 \\
2732.92 \\
27232.17\end{array}$ & $\begin{array}{l}5 \\
1\end{array}$ & $\begin{array}{l}15 \mathrm{Hl} \\
25 \\
5 \mathrm{Hl} \\
5 \\
10 \mathrm{Hl}\end{array}$ & V $E$ & $\begin{array}{l}36562.03 \\
36566.90 \\
36579.02 \\
36580.09 \\
36590.13\end{array}$ & $\begin{array}{l}a^{5} \mathrm{D}_{2}-z^{5} \mathrm{~F}_{2}^{\circ} \\
a^{5} \mathrm{D}_{4}-z^{5} \mathrm{~F}_{3}^{0}\end{array}$ & & \\
\hline $\begin{array}{l}2731.12 \\
2730.60 \\
2729.64 \\
2728.644 \\
2727.929\end{array}$ & $\begin{array}{r}20 \\
1\end{array}$ & $\begin{array}{c}4 H \\
2 h \\
1 \\
150 \\
6\end{array}$ & V $E$ & $\begin{array}{l}36604.20 \\
36611.17 \\
36624.04 \\
36637.41 \\
36647.02\end{array}$ & $\begin{array}{l}a^{3} \mathrm{G}_{3}-y^{3} \mathrm{D}_{3}^{\circ} \\
a^{5} \mathrm{D}_{1}-z{ }^{5} \mathrm{~F}_{2}^{0} \\
a^{3} \mathrm{H}_{5}-y^{3} \mathrm{~F}_{4}\end{array}$ & & \\
\hline $\begin{array}{l}2726.544^{*} \\
2724.61 \\
2724.40 \\
2723.455 \\
2723.218\end{array}$ & 5 & $\begin{array}{l}40 \\
5 H \\
2 \\
10 \\
20\end{array}$ & VE & $\begin{array}{l}36665.63 \\
36691.65 \\
36694.48 \\
36707.22 \\
36710.41\end{array}$ & $a^{5} \mathrm{D}_{3}-z^{5} \mathrm{~F}_{3}^{\circ}$ & & \\
\hline $\begin{array}{l}2722.258 \\
2722.095 \\
2720.77 \\
2717.464\end{array}$ & & $\begin{array}{l}3 \\
1 \\
2 \\
5\end{array}$ & & $\begin{array}{l}36723.35 \\
36725.55 \\
36743.44 \\
36788.13\end{array}$ & $\begin{array}{l}a^{3} \mathrm{H}_{4}-y{ }^{3} \mathrm{~F}_{4}^{\circ} \\
b^{3} \mathrm{D}_{2}-y \\
a^{3} \mathrm{D}_{2} \\
a^{3}-z{ }^{1} \mathrm{~S}_{0}^{\circ} \\
a^{3} \mathrm{D}_{3}-y \\
{ }^{1} \mathrm{~F}_{3}^{2}\end{array}$ & & \\
\hline 2715.676 & $30 r$ & $180 \mathrm{rs}$ & V $E$ & 36812.35 & $\left\{\begin{array}{l}a^{5}{ }^{5} \mathrm{D}_{2}-z \\
a^{5} \mathrm{D}_{4}{ }^{5} \mathrm{~F}_{3}^{3} \\
{ }^{5} \mathrm{D}_{4}\end{array}\right.$ & & \\
\hline
\end{tabular}

See footnotes at end of table. 
TABLE 1.-Second spectrum of Vanadium-Continued

\begin{tabular}{|c|c|c|c|c|c|c|c|}
\hline \multirow{3}{*}{$\lambda_{\mathrm{air}} \mathrm{A}$} & \multicolumn{2}{|c|}{2} & 3 & 4 & 5 & \multicolumn{2}{|c|}{6} \\
\hline & \multicolumn{2}{|c|}{ Intensity } & \multirow{2}{*}{$\begin{array}{c}\text { Temp. } \\
\text { class }\end{array}$} & \multirow{2}{*}{$\begin{array}{l}\text { Wave } \\
\text { No. vac }\end{array}$} & \multirow{2}{*}{$\begin{array}{c}\text { Term } \\
\text { combination }\end{array}$} & \multicolumn{2}{|c|}{ Zeeman effect } \\
\hline & Are & Spark & & & & Observed & Computed \\
\hline $\begin{array}{l}2714.42 \\
2714.205 \\
2713.050 \\
2712.81 \\
2712.21\end{array}$ & $\begin{array}{r}10 \\
8\end{array}$ & $\begin{array}{l}10 H \\
50 \\
40 \\
7 H l \\
30 H l\end{array}$ & $\begin{array}{l}\mathrm{V} E \\
\mathrm{~V} E\end{array}$ & $\begin{array}{l}36829.39 \\
36832.30 \\
36847.98 \\
36851.24 \\
36859.39\end{array}$ & $\begin{array}{l}a{ }^{5} \mathrm{D}_{3}-z{ }^{3} \mathrm{D}_{2}^{\circ} \\
a^{5} \mathrm{D}_{2}-z{ }^{3} \mathrm{D}_{1}^{2}\end{array}$ & & \\
\hline $\begin{array}{l}2711.740 \\
2710.17 \\
2709.10 \\
2708.91 \\
2707.86\end{array}$ & $\begin{array}{r}15 \\
2\end{array}$ & $\begin{array}{c}100 \\
15 \\
6 H \\
1 ? \\
100\end{array}$ & $\begin{array}{l}\text { V } E \\
\text { VE }\end{array}$ & $\begin{array}{l}36865.78 \\
36887.14 \\
36901.70 \\
36904.29 \\
36918.60\end{array}$ & $\begin{array}{l}a^{5}{ }^{5} \mathrm{D}_{4}-z^{3} \mathrm{D}_{3}^{0} \\
a^{3} \mathrm{H}_{6}-z^{1} \mathrm{H}_{5}^{8} \\
\\
d^{3} \mathrm{P}_{2}-u^{3} \mathrm{3}_{3}^{3} \\
a^{5} \mathrm{D}_{1}-z^{3} \mathrm{D}_{1}^{\circ}\end{array}$ & & \\
\hline $\begin{array}{l}2706.70 \\
2706.17 \\
2705.220 \\
2703.15 \\
2702.185\end{array}$ & $\begin{array}{r}20 \\
30 \\
7 \\
30\end{array}$ & $\begin{array}{c}150 r \\
200 r \\
40 \\
3 \\
200 r\end{array}$ & $\begin{array}{l}\mathrm{V} E \\
\mathrm{~V} E r s \\
\mathrm{~V} E \\
\mathrm{~V} E\end{array}$ & $\begin{array}{l}36934.42 \\
36941.66 \\
36954.63 \\
36982.93 \\
36996.13\end{array}$ & $\begin{array}{l}a^{5} \mathrm{D}_{2}-z^{3} \mathrm{D}_{2}^{\circ} \\
a^{5} \mathrm{D}_{3}-z^{5} \mathrm{~F}_{4}^{\circ} \\
a^{5} \mathrm{D}_{0}-z^{3} \mathrm{D}_{1}^{\circ} \\
b^{3} \mathrm{~F}_{2}-y^{3} \mathrm{D}_{1}^{\circ} \\
a^{5} \mathrm{D}_{3}-z^{3} \mathrm{D}_{3}^{\circ}\end{array}$ & & \\
\hline $\begin{array}{l}2701.535 \\
2700.944 \\
2700.20 \\
2699.63 \\
2697.201\end{array}$ & $\begin{array}{r}4 \\
40\end{array}$ & $\begin{array}{c}10 \\
300 r \\
1 ? \\
4 H l \\
10\end{array}$ & $\begin{array}{l}\text { V } \\
\text { V Ers }\end{array}$ & $\begin{array}{l}37005.03 \\
37013.13 \\
37023.33 \\
37031.14 \\
37064.49\end{array}$ & $\begin{array}{l}a^{5} \mathrm{D}_{1}-z^{3} \mathrm{D}_{2}^{\circ} \\
a^{5} \mathrm{D}_{4}-z^{5} \mathrm{~F}_{5}^{\circ} \\
a^{3} \mathrm{H}_{4}-z^{1} \mathrm{~F}_{3}^{0} \\
d^{3} \mathrm{~F}_{2}-v^{3} \mathrm{~F}_{2}^{\circ}\end{array}$ & & \\
\hline $\begin{array}{l}2696.51 \\
2694.74^{*} \\
2694.65 \\
2694.47 \\
2693.00\end{array}$ & $\begin{array}{l}1 \\
2\end{array}$ & $\begin{array}{c}20 H \\
20 H \\
10 H \\
5 \\
5 H+\mathrm{I}\end{array}$ & & $\begin{array}{l}37073.99 \\
37098.34 \\
37099.58 \\
37102.06 \\
37122.31\end{array}$ & $\begin{array}{l}a{ }^{5} \mathrm{D}_{2}-z^{3} \mathrm{D}_{3}^{\circ} \\
a^{3} \mathrm{H}_{5}-z^{1} \mathrm{G}_{4}^{\circ}\end{array}$ & & \\
\hline $\begin{array}{l}2690.792 \\
2690.252 \\
2689.883 \\
2688.717 \\
2687.960\end{array}$ & $\begin{array}{l}20 \\
15 \\
10 \\
10 \\
60 r\end{array}$ & $\begin{array}{l}200 \\
150 \\
100 \\
100 \\
300 r\end{array}$ & $\begin{array}{ll}\text { V } & E a \\
\text { V } & E a \\
\text { V } & E a \\
\text { V } & E a \\
\text { V } & E a\end{array}$ & $\begin{array}{l}37152.77 \\
37160.22 \\
37165.32 \\
37181.44 \\
37191.91\end{array}$ & $\begin{array}{l}a^{5}{ }^{5} \mathrm{D}_{2}-z^{5} \mathrm{D}_{1}^{0} \\
a^{5} \mathrm{D}_{3}-z^{5} \mathrm{D}_{2}^{2} \\
a^{5} \mathrm{D}_{1}-z^{5} \mathrm{D}_{0}^{0} \\
a^{5} \mathrm{D}_{4}-z^{5} \mathrm{D}_{3}^{3} \\
a^{5} \mathrm{D}_{4}-z^{5} \mathrm{D}_{4}^{8}\end{array}$ & & \\
\hline 2685. 689 & 4 & 30 & & 37223.36 & $a^{5} \mathrm{D}_{1}-z^{5} \mathrm{D}_{1}$ & & \\
\hline 2685.41 & & $1 ?$ & & 37227.22 & $\begin{array}{l}a^{5} \mathrm{P}_{1}-y^{3} \mathrm{P}_{1}^{1} \\
a^{5} \mathrm{~F}_{4}-z^{3} \mathrm{~F}_{3}^{0}\end{array}$ & & \\
\hline $\begin{array}{l}2685.138 \\
2684.78 \\
2683.09\end{array}$ & $\begin{array}{l}10 \\
15\end{array}$ & $\begin{array}{l}20+I \\
15 H \\
100\end{array}$ & $\begin{array}{l}\text { V } E \\
\text { V } E a\end{array}$ & $\begin{array}{l}37230.99 \\
37235.96 \\
37259.41\end{array}$ & $\begin{array}{l}a^{1} \mathrm{G}_{4}-y^{1} F_{3}^{\circ} \\
a^{5} \mathrm{D}_{0}-z{ }^{5} \mathrm{D}^{\circ}\end{array}$ & & 1 \\
\hline $\begin{array}{l}2682.875 \\
2682.535 \\
2681.29\end{array}$ & $\begin{array}{r}15 \\
1\end{array}$ & $\begin{array}{r}100 \\
6 \\
1\end{array}$ & V $E a$ & $\begin{array}{l}37262.40 \\
37267.12 \\
37284.42\end{array}$ & $\begin{array}{l}a^{5} \mathrm{D}_{2}-z^{5} \mathrm{D}_{2}^{2} \\
a^{5} \mathrm{~F}_{5}-z{ }^{3} \mathrm{~F}_{4}^{\circ} \\
b^{3} \mathrm{~F}_{2}-y^{3} \mathrm{D}_{2}^{\circ} ?\end{array}$ & & \\
\hline 2680.822 & & 1 & & 37290.93 & $\left\{\begin{array}{l}a{ }^{1} \mathrm{~F}_{3}-w^{3} \mathrm{G}_{4}^{\circ} \\
d^{3} \mathrm{BF}_{4}-v^{3} \mathrm{~F}_{3}^{\circ}\end{array}\right.$ & & \\
\hline 2680.470 & 1 & 8 & & 37295.83 & a $1 \mathrm{G}_{4}-x{ }^{3} \mathrm{G}_{5}^{\circ}$ & (0) 1.64 & (0) $1.55 b$ \\
\hline $\begin{array}{l}2679.327 \\
2678.572 \\
2677.804 \\
2677.00 \\
2676.33\end{array}$ & $\begin{array}{l}30 \\
10 \\
25\end{array}$ & $\begin{array}{c}200 r \\
100 r \\
150 r \\
1 \\
7 H\end{array}$ & $\begin{array}{l}\text { V Ea } \\
\text { V Ea }\end{array}$ & $\begin{array}{l}37311.74 \\
37322.25 \\
37332.95 \\
37344.17 \\
37353.52\end{array}$ & $\begin{array}{l}a^{5}{ }^{5} \mathrm{D}_{3}-z{ }^{5} \mathrm{D}_{3}^{\circ} \\
a^{5} \mathrm{D}_{3}-z{ }^{5} \mathrm{D}_{4}^{\circ} \\
a^{5} \mathrm{D}_{1}-z D^{5} \mathrm{D}_{2}^{6} \\
a^{5} \mathrm{P}_{3}-y{ }^{3} \mathrm{D}_{3}^{\circ} \\
z^{5} \mathrm{G}_{6}^{6}-e^{5} \mathrm{H}_{6}\end{array}$ & & \\
\hline $\begin{array}{l}2676.05 \\
2675.17 \\
2674.28 \\
2673.955 \\
2673.25\end{array}$ & $\begin{array}{l}1 \\
1\end{array}$ & $\begin{array}{l}9 H \\
2 h \\
3 h \\
4 \\
50 H\end{array}$ & & $\begin{array}{l}37357.42 \\
37369.71 \\
37382.15 \\
37386.69 \\
37396.55\end{array}$ & $\begin{array}{l}z^{5} \mathrm{G}_{5}^{\circ}-e{ }^{5} \mathrm{H}_{4} \\
a^{5} \mathrm{~F}_{3}-z^{5} \mathrm{~F}_{3}^{\circ}\end{array}$ & & \\
\hline $\begin{array}{l}2672.005 \\
2670.237 \\
2668.595 \\
2668.01 \\
2667.532\end{array}$ & $\begin{array}{r}20 \\
2\end{array}$ & $\begin{array}{c}150 r \\
40 \\
4 \\
10 H \\
4\end{array}$ & V $E a$ & $\begin{array}{l}37413.97 \\
37438.74 \\
37461.78 \\
37469,99 \\
37476.71\end{array}$ & $\begin{array}{l}a{ }^{5} \mathrm{D}_{2}-z^{5} \mathrm{D}_{3}^{0} \\
a^{1} \mathrm{G}_{4}-x^{3} \mathrm{G}_{3}^{3} \\
a^{5} \mathrm{~F}_{4}-z^{3} \mathrm{~F}^{8} \\
c^{3} \mathrm{~F}_{2}-v^{3} \mathrm{~F}_{2}^{2} \\
b^{3} \mathrm{~F}_{4}-y^{3} \mathrm{D}_{3}^{0}\end{array}$ & & \\
\hline $\begin{array}{l}2666.79 \\
2666.50 \\
2665.490 \\
2665.277 \\
2663.837\end{array}$ & & $\begin{array}{l}10 H \\
1 \\
2 \\
3 \\
2 h\end{array}$ & & $\begin{array}{l}37487.13 \\
37491.21 \\
37505.42 \\
37508.41 \\
37528.69\end{array}$ & $\begin{array}{l}z^{5} \mathrm{G}_{5} 0-e^{5} \mathrm{H}_{5} \\
a^{5} \mathrm{P}_{3}-y^{3} \mathrm{D}_{3}^{\circ} \\
d^{3} \mathrm{~F}_{3}-v^{3} \mathrm{~F}_{4}^{0} \\
a^{5} \mathrm{~F}_{2}-z^{3} \mathrm{~F}_{3}^{0} \\
a^{5} \mathrm{P}_{2}-y^{3} \mathrm{P}_{2}^{\circ}\end{array}$ & & \\
\hline
\end{tabular}

See footnotes at end of table. 
TABLE 1.-Second spectrum of Vanadium-Continued

\begin{tabular}{|c|c|c|c|c|c|c|c|}
\hline 1 & & 2 & 3 & 4 & 5 & & \\
\hline \multirow{2}{*}{$\lambda_{\mathrm{a} \text { ir } \mathrm{A}}$} & \multicolumn{2}{|c|}{ Intensity } & \multirow{2}{*}{$\begin{array}{c}\text { Temp. } \\
\text { class }\end{array}$} & \multirow{2}{*}{$\begin{array}{l}\text { Wave } \\
\text { No. vac }\end{array}$} & \multirow{2}{*}{$\begin{array}{c}\text { Term } \\
\text { combination }\end{array}$} & \multicolumn{2}{|c|}{ Zeeman effect } \\
\hline & Arc & Spark & & & & Observed & Computed \\
\hline $\begin{array}{l}2663.526 \\
2663.25 \\
2661.47 \\
2661.243 \\
2659.60\end{array}$ & 2 & $\begin{array}{c}4 h \\
250 H \\
30 H \\
3 \\
25\end{array}$ & & $\begin{array}{l}37533.07 \\
37536.96 \\
37562.06 \\
37565.27 \\
37588.47\end{array}$ & $\begin{array}{l}d^{3} \mathrm{~F}_{4}-v{ }^{3} \mathrm{~F}_{4}^{\circ} \\
z^{3} \mathrm{G}_{6}^{\circ}-e^{3} \mathrm{H}_{7} \\
a^{1} \mathrm{G}_{4}-y{ }^{1} \mathrm{H}_{5}^{\circ}\end{array}$ & & \\
\hline 2658.97 & $3 d$ & 30 & & 37597.37 & $\left\{\begin{array}{l}a^{3} \mathrm{G}_{4}-y{ }^{3} \mathrm{H}_{5}^{\circ} \\
a^{3} \mathrm{G}_{5}-y^{3} \mathrm{H}_{6}^{\circ}\end{array}\right.$ & & \\
\hline $\begin{array}{l}2658.49 \\
2657.97 \\
2657.295 \\
2655.68\end{array}$ & $\begin{array}{l}1 \\
2 e\end{array}$ & $\begin{array}{c}7 \mathrm{Hl} \\
1 \\
10 \\
200 \mathrm{H}\end{array}$ & & $\begin{array}{l}37604.16 \\
37611.52 \\
37621.07 \\
37643.95\end{array}$ & $\begin{array}{l}z^{5} \mathrm{G}_{4}{ }^{\circ}-e{ }^{5} \mathrm{H}_{4} \\
a^{5} \mathrm{P}_{1}-y{ }^{3} \mathrm{P}_{2}^{0} \\
a^{3} \mathrm{G}_{3}-y{ }^{3} \mathrm{H}_{4}^{\circ} \\
z^{5} \mathrm{G}_{5}^{\circ}-e^{5} \mathrm{H}_{8}\end{array}$ & & \\
\hline $\begin{array}{l}2654.89 \\
2654.39 \\
2652.76 \\
2651.57 \\
2650.41\end{array}$ & & $\begin{array}{l}2 \\
3 H \\
20 H \\
5 H \\
3 h\end{array}$ & & $\begin{array}{l}37655.15 \\
37662.24 \\
37685.38 \\
37702.30 \\
37718.80\end{array}$ & $z^{5} \mathrm{G}_{3}^{0}-e^{5} \mathrm{H}_{3}$ & & \\
\hline $\begin{array}{l}2650.136 \\
2649.37 \\
2648.475 \\
2646.225 \\
2645.840\end{array}$ & $\begin{array}{l}1 e \\
1 \\
6\end{array}$ & $\begin{array}{c}1 \\
150 H \\
30 \\
2 \\
80\end{array}$ & & $\begin{array}{l}37722.70 \\
37733.60 \\
37746.35 \\
37778.44 \\
37783.94\end{array}$ & $\begin{array}{l}b^{3} \mathrm{G}_{4}-y^{1} \mathrm{G}_{4}^{\circ} \\
z^{5} \mathrm{G}_{4}^{\circ}-e^{5} \mathrm{H}_{5} \\
a^{1} \mathrm{~F}_{3}-x{ }^{1} \mathrm{D}_{2}^{\circ} \\
a^{3} \mathrm{G}_{3}-x^{3} \mathrm{~F}_{2}^{\circ}\end{array}$ & & \\
\hline $\begin{array}{l}2644.363 \\
2643.69 \\
2643.11 \\
2642.72 \\
2642.212^{\text {i }}\end{array}$ & 5 & $\begin{array}{l}100 \mathrm{Hl} \\
4 \mathrm{H} \\
2 \\
6 \\
80\end{array}$ & & $\begin{array}{l}37805.04 \\
37814.67 \\
37822.96 \\
37828.55 \\
37835.82\end{array}$ & $\begin{array}{l}z^{5} \mathrm{G}_{3}-e^{5} \mathrm{H}_{4} \\
a^{3} \mathrm{P}_{2}-z^{5} \mathrm{~S}_{2}^{\circ} \\
c^{3} \mathrm{~F}_{4}-v^{3} \mathrm{~F}_{4}^{0} \\
a^{3} \mathrm{G}_{4}-x^{3} \mathrm{~F}_{3}^{0}\end{array}$ & $\begin{array}{l}(0) 0.48 \\
2 p ; n w_{2} \mathrm{~A}\end{array}$ & \\
\hline $\begin{array}{l}2640.86 \\
2639.29 \\
2638.74 \\
2638.52 \\
2637.886\end{array}$ & & $\begin{array}{l}80 \mathrm{H} \\
2 \\
2 \\
7 \mathrm{H} \\
2\end{array}$ & & $\begin{array}{l}37855.19 \\
37877.70 \\
37885.60 \\
37888.76 \\
37897.86\end{array}$ & $\begin{array}{l}z^{{ }^{5} \mathrm{G}_{2}^{\circ}-e} e^{5} \mathrm{H}_{3} \\
a^{3} \mathrm{D}_{2}-y^{1} \mathrm{P}_{1}^{\circ} \\
a^{3} \mathrm{~F}_{3}-z^{3} \mathrm{P}_{2}^{\circ}\end{array}$ & & \\
\hline $\begin{array}{l}2637.623 \\
2637.25 \\
2636.40 \\
2636.00 \\
2635.640\end{array}$ & 1 & $\begin{array}{l}2 h \\
6 H \\
2 H \\
5 H \\
10\end{array}$ & & $\begin{array}{l}37901.64 \\
37907.00 \\
37919.22 \\
37924.98 \\
37930.16\end{array}$ & $\begin{array}{l}z^{5} \mathrm{G}_{2}^{\circ}-e^{5} \mathrm{P}_{1} \\
a^{3} \mathrm{G}_{3}-x^{3} \mathrm{~F}_{3}^{0}\end{array}$ & & \\
\hline $\begin{array}{l}2635.43 \\
2634.37 \\
2633.91 \\
2633.28 \\
2632.96\end{array}$ & & $\begin{array}{l}7 H \\
3 H \\
3 H \\
2 H ? \\
3 H\end{array}$ & & $\begin{array}{l}37933.18 \\
37948.44 \\
37955.07 \\
37964.15 \\
37968.76\end{array}$ & $\begin{array}{l}{ }^{5} \mathrm{G}_{6}^{\circ}-e^{5} \mathrm{G}_{5} \\
a^{1} \mathrm{~F}_{3}-v^{3} \mathrm{D}_{2}^{\circ}\end{array}$ & & \\
\hline $\begin{array}{l}2631.484 \\
2630.665 \\
2629.72^{\mathrm{i}} \\
2628.75^{\mathrm{m}} \\
2628.09^{3}\end{array}$ & 10 & $\begin{array}{c}3 \\
150 \\
60 H \\
30 H \\
4 H\end{array}$ & & $\begin{array}{l}37990.06 \\
38001.88 \\
38015.54 \\
38029.56 \\
38039.11\end{array}$ & $\begin{array}{l}a^{3} \mathrm{P}_{2}-z^{1} \mathrm{D}_{2}^{\circ} \\
a^{3} \mathrm{G}_{5}-x^{3} \mathrm{~F}_{4}^{0} \\
z^{5} \mathrm{G}_{6}^{\circ}-e^{5} \mathrm{G}_{6}\end{array}$ & $\begin{array}{l}\text { (?) } 3 ? p ; n w_{2} \mathrm{~B} \\
\text { (0) } n w_{1} \\
\text { (1.06) } 1.83\end{array}$ & (0) $1.15 b$ \\
\hline $\begin{array}{l}2627.32 \\
2626.42 \\
2625.01 \\
2624.860 \\
2623.792\end{array}$ & 1 & $\begin{array}{l}1 \\
2 H ? \\
4 H ? \\
15 H \\
15+H\end{array}$ & & $\begin{array}{l}38050.26 \\
38063.30 \\
38083.74 \\
38085.92 \\
38101.42\end{array}$ & $\begin{array}{l}a^{3} \mathrm{~F}_{2}-z^{3} \mathrm{P}_{1}^{\circ} \\
b^{1} \mathrm{~F}_{3}-w^{1} \mathrm{G}_{4} \\
z^{5} \mathrm{G}_{5}^{\circ}-e^{5} \mathrm{G}_{4} \\
a^{3} \mathrm{G}_{4}-x^{3} \mathrm{~F}_{4}^{0}\end{array}$ & & \\
\hline $\begin{array}{l}2622.74 \\
2621.80 \\
2620.06^{1} \\
2619.48 \\
2618.63\end{array}$ & $2 ?$ & $\begin{array}{l}50 H \\
40 H \\
3 \\
5 H \\
1 h ?\end{array}$ & & $\begin{array}{l}38116.71 \\
38130.37 \\
38155.69 \\
38164.14 \\
38176.52\end{array}$ & $b^{3} \mathrm{P}_{1}-y{ }^{1} \mathrm{D}_{2}^{2}$ & $\begin{array}{l}\text { (?) } 0.92 n w_{1} \mathrm{~A} \\
\text { (0) } 2.29\end{array}$ & \\
\hline $\begin{array}{l}2618.40 \\
2617.10 \\
2616.66 \\
2616.24 \\
2615.89\end{array}$ & 3 & $\begin{array}{c}3 H \\
9 H \\
10 H \\
40 \\
2\end{array}$ & & $\begin{array}{l}38179.88 \\
38198.84 \\
38205.27 \\
38211.40 \\
38216.51\end{array}$ & $\begin{array}{l}z^{5} \mathrm{G}_{4}^{\circ}-e^{5} \mathrm{G}_{3} \\
z^{5} \mathrm{G}_{3}^{0}-e^{5} \mathrm{D}_{4} ? \\
a^{3} \mathrm{G}_{4}-x^{3} \mathrm{D}_{3}^{\circ} \\
a^{3} \mathrm{P}_{1}-z^{5} \mathrm{~S}_{2}^{0}\end{array}$ & & \\
\hline
\end{tabular}

See footnotes at end of table. 
TABLE 1.-Second spectrum of Vanadium-Continued

\begin{tabular}{|c|c|c|c|c|c|c|c|}
\hline \multirow{3}{*}{$\lambda_{\mathrm{air}} \mathrm{A}$} & \multicolumn{2}{|c|}{2} & 3 & 4 & 5 & \multicolumn{2}{|c|}{6} \\
\hline & \multicolumn{2}{|c|}{ Intensity } & \multirow{2}{*}{$\begin{array}{l}\text { Temp. } \\
\text { class }\end{array}$} & \multirow{2}{*}{$\begin{array}{l}\text { Wave } \\
\text { No. vac }\end{array}$} & \multirow{2}{*}{$\begin{array}{c}\text { Term } \\
\text { combination }\end{array}$} & \multicolumn{2}{|c|}{ Zeeman effect } \\
\hline & Arc & Spark & & & & Observed & Computed \\
\hline $\begin{array}{l}2615.401 \\
2614.395 \\
2612.26 \\
2611.51 \\
2611.24\end{array}$ & 1 & $\begin{array}{l}40 H \\
10 \\
15 H \\
7 H \\
10 H\end{array}$ & & $\begin{array}{l}38223.67 \\
38238.36 \\
38269.61 \\
38280.60 \\
38284.56\end{array}$ & $\begin{array}{l}z^{5} \mathrm{G}_{5}^{\circ}-e^{5} \mathrm{G}_{5} \\
a^{3} \mathrm{G}_{3}-x^{3} \mathrm{D}_{2}^{\circ} \\
z^{5} \mathrm{G}_{3}^{\circ}-e^{5} \mathrm{G}_{2}\end{array}$ & (0) 2.33 & \\
\hline 2610.61 & & $30 H$ & & 38293.80 & & & \\
\hline 2609.80 & & 5 & & 38305.68 & $\left\{\begin{array}{l}a^{3}{ }^{3} \mathrm{G}_{3}-x{ }^{3} \mathrm{D}_{3}^{2} \\
z^{5} \mathrm{G}^{\circ}-e^{5} \mathrm{G}^{3}\end{array}\right.$ & & \\
\hline $\begin{array}{l}2609.60 \\
2608.66 \\
2608.00\end{array}$ & & $\begin{array}{c}3 \\
1 \\
20 H\end{array}$ & & $\begin{array}{l}38308.62 \\
38322.42 \\
38332.12\end{array}$ & $\begin{array}{l}a{ }^{3} \mathrm{~F}_{4}-y \\
z^{5} \mathrm{G}_{4}{ }^{5}-e^{5} \mathrm{D}_{4}\end{array}$ & & \\
\hline $\begin{array}{l}2607.41 \\
2605.70 \\
2604.51 \\
2603.40 \\
2602.94\end{array}$ & & $\begin{array}{c}3 H \\
7 H \\
1 \\
15 H \\
15 H\end{array}$ & & $\begin{array}{l}38340.79 \\
38365.95 \\
38383.48 \\
38399.84 \\
38406.63\end{array}$ & $\begin{array}{l}a^{3} \mathrm{P}_{1}-z^{1} \mathrm{D}_{2}^{\circ} \\
z^{5} \mathrm{G}_{3}^{\circ}-e^{5} \mathrm{G}_{3}\end{array}$ & & \\
\hline $\begin{array}{l}2602.32 \\
2601.08 \\
2600.60 \\
2600.03 \\
2598.65\end{array}$ & & $\begin{array}{l}6 H \\
25 H \\
4 H \\
2 H ? \\
2 H\end{array}$ & & $\begin{array}{l}38415.78 \\
38434.09 \\
38441.19 \\
38449.61 \\
38470.03\end{array}$ & $\begin{array}{l}z^{5} \mathrm{G}_{2}-e^{5} \mathrm{G}_{2} \\
z^{5} \mathrm{G}_{4}-e^{5} \mathrm{G}_{5}\end{array}$ & & \\
\hline $2597.21^{k, n}$ & & $6 h$ & & 38491.36 & $\left\{\begin{array}{l}c^{3} \mathrm{~F}_{3}-u^{3} \mathrm{D}_{2}^{\circ} \\
c^{3} \mathrm{~F}_{2}-u^{3} \mathrm{D}_{1}^{0}\end{array}\right\}$ & (0) 2.36 & \\
\hline $\begin{array}{l}2594.43 \\
2593.91 \\
2593.64 \\
2592.215\end{array}$ & & $\begin{array}{l}3 h \\
2 H \\
1 ? \\
4\end{array}$ & & $\begin{array}{l}38532.60 \\
38540.32 \\
38544.33 \\
38565.52\end{array}$ & $\begin{array}{l}z^{5} \mathrm{G}_{3}^{0}-e^{5} \mathrm{G}_{4} \\
b^{3} \mathrm{~F}_{3}-y^{3} \mathrm{H}_{4}^{\circ} \\
b^{3} \mathrm{~F}_{4}-y^{3} \mathrm{H}_{5}^{\circ} ? \\
a^{3} \mathrm{P}_{2}-y^{3} \mathrm{D}_{1}^{\circ}\end{array}$ & & \\
\hline $\begin{array}{l}2591.10 \\
2590.55 \\
2590.17 \\
2588.789 \\
2588.48\end{array}$ & & $\begin{array}{l}3 H \\
5 H \\
4 H \\
3 h \\
3 H\end{array}$ & & $\begin{array}{l}38582.12 \\
38590.31 \\
38595.97 \\
38616.56 \\
38621.17\end{array}$ & & & \\
\hline $\begin{array}{l}2588.128 \\
2587.37 \\
2585.87 \\
2584.951 \\
2583.58\end{array}$ & $\begin{array}{l}2 \\
3\end{array}$ & $\begin{array}{c}3 \\
5 H \\
10+\mathrm{Fe} \\
80 \\
2 H\end{array}$ & & $\begin{array}{l}38626.42 \\
38637.73 \\
38660.15 \\
38673.89 \\
38694.41\end{array}$ & $\begin{array}{l}c^{3} \mathrm{~F}_{4}-u^{3} \mathrm{D}_{3}^{\circ} \\
b^{3} \mathrm{G}_{5}-x^{3} \mathrm{G}_{5}^{\circ}\end{array}$ & & \\
\hline $\begin{array}{l}2583.007 \\
2581.839 \\
2578.451 \\
2577.682\end{array}$ & 3 & $\begin{array}{r}20 \\
4 \\
8 \\
40\end{array}$ & & $\begin{array}{l}38702.99 \\
38720.50 \\
38771.37 \\
38782.94\end{array}$ & $\begin{array}{l}b^{3} \mathrm{~F}_{3}-x{ }^{3} \mathrm{~F}_{2}^{0} \\
b^{3} \mathrm{G}_{4}-y{ }^{1} \mathrm{~F}_{3}^{0} \\
b^{3} \mathrm{G}_{5}-x{ }^{3} \mathrm{G}_{4}^{0} \\
b^{3} \mathrm{~F}_{4}-x{ }^{3} \mathrm{~F}_{3}^{0}\end{array}$ & & \\
\hline 2577.528 & & 3 & & 38785.26 & $\left\{\begin{array}{l}a{ }^{1} \mathrm{H}_{5}-w^{3} \mathrm{~F}_{4}^{3} \\
b^{3} \mathrm{G}_{4}-x^{3} \mathrm{G}_{5}^{8}\end{array}\right.$ & & \\
\hline $\begin{array}{l}2576.478 \circ \\
2574.520 \\
2573.20 \\
2572.71 \\
2572.096\end{array}$ & $\begin{array}{l}1 \\
4\end{array}$ & $\begin{array}{l}20 \\
60 \\
4 H \\
4 H \\
2\end{array}$ & & $\begin{array}{l}38801.06 \\
38830.57 \\
38850.49 \\
38857.89 \\
38867.16\end{array}$ & $\begin{array}{l}b^{3} \mathrm{G}_{3}-y{ }^{1} \mathrm{~F}_{3}^{\circ} \\
a^{3} \mathrm{P}_{2}-y \\
{ }^{3} \mathrm{P}_{1}^{\circ} \\
a^{3} \mathrm{P}_{2}-y{ }^{3} \mathrm{D}_{2}^{\circ}\end{array}$ & $(0.41) ?$ & $(0.46) 0.85$ \\
\hline $\begin{array}{l}2571.059 \\
2568.31 \\
2568.065 \\
2567.45 \\
2566.602\end{array}$ & 2 & $\begin{array}{c}50 \\
3 \\
3 \\
15 H \\
15\end{array}$ & & $\begin{array}{r}38882.84 \\
538924.45 \\
38928.17 \\
38937.49 \\
38950.36\end{array}$ & $\begin{array}{l}b^{3} \mathrm{G}_{4}-x^{3} \mathrm{G}_{4}^{\circ} \\
b^{3} \mathrm{G}_{4}-x^{3} \mathrm{G}_{3}^{\circ} \\
a^{1} \mathrm{~F}_{3}-x^{1} \mathrm{G}_{4}^{\circ}\end{array}$ & & \\
\hline $\begin{array}{l}2566.033 \\
2565.743 \\
2565.543 \\
2565.24 \\
2562.760\end{array}$ & $\begin{array}{l}1 \\
1 \\
1\end{array}$ & $\begin{array}{r}7 \\
2 \\
15 \\
3 \\
30\end{array}$ & & $\begin{array}{l}38958.99 \\
38963.39 \\
38966.43 \\
38971.03 \\
39008.74\end{array}$ & $\begin{array}{l}a^{3} \mathrm{P}_{1}-y{ }^{3} \mathrm{D}_{1}^{\mathrm{i}} \\
b^{3} \mathrm{G}_{3}-x^{3} \mathrm{G}_{4}^{\circ} \\
b^{3} \mathrm{G}_{5}-y{ }^{1} \mathrm{H}_{5}^{\circ} \\
d^{3} \mathrm{~F}_{2}-v^{3} \mathrm{G}_{3}^{\circ} \\
b^{3} \mathrm{G}_{3}-x^{3} \mathrm{G}_{3}^{\circ}\end{array}$ & & \\
\hline $\begin{array}{l}2561.65 \\
\\
2560.149 \\
2559.088 \\
2556.43 \\
2555.905\end{array}$ & 2 & $\begin{array}{r}2 h \\
4 \\
2 \\
1 \\
40\end{array}$ & & $\begin{array}{l}39025.65 \\
\\
39048.53 \\
39064.71 \\
39105.33 \\
39113.36\end{array}$ & 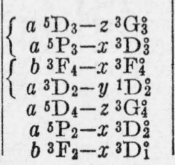 & & \\
\hline
\end{tabular}

See footnotes at end of table. 
TABLE 1.-Second spectrum of Vanadium-Continued

\begin{tabular}{|c|c|c|c|c|c|c|c|}
\hline \multirow{3}{*}{$\lambda_{\text {air }} \mathrm{A}$} & \multicolumn{2}{|c|}{2} & 3 & 4 & 5 & & \\
\hline & & nsity & \multirow{2}{*}{$\begin{array}{l}\text { Temp. } \\
\text { class }\end{array}$} & \multirow{2}{*}{$\begin{array}{l}\text { Wave } \\
\text { No. vac }\end{array}$} & \multirow{2}{*}{$\begin{array}{c}\text { Term } \\
\text { combination }\end{array}$} & \multicolumn{2}{|c|}{ Zeeman effect } \\
\hline & Arc & Spark & & & & Observed & Computed \\
\hline $\begin{array}{l}2554.22 \\
2554.06 \\
2553.668 \\
2553.028 \\
2552.960\end{array}$ & $\begin{array}{l}4 \\
3 \\
4\end{array}$ & $\begin{array}{l}15 H \\
10 H \\
40 \\
40 \\
60\end{array}$ & & $\begin{array}{l}39139.16 \\
39141.61 \\
39147.62 \\
39157.43 \\
39158.48\end{array}$ & $\begin{array}{l}a{ }^{3} \mathrm{P}_{1}-y{ }^{3} \mathrm{P}_{0}^{0} \\
b^{3} \mathrm{~F}_{3}-x \\
b^{3} \mathrm{~B}_{4} \mathrm{D}_{2}^{2}-x^{3} \mathrm{D}_{3}^{3}\end{array}$ & & \\
\hline $\begin{array}{l}2552.264 \\
2551.724 \\
2550.580 \\
2549.653 \\
2549.272\end{array}$ & 8 & $\begin{array}{c}2 h \\
15 \\
3 \\
10 \\
120\end{array}$ & & $\begin{array}{l}39169.15 \\
39177.44 \\
39195.01 \\
39209.26 \\
39215.12\end{array}$ & $\begin{array}{c}a^{3}{ }^{3} \mathrm{P}_{2}-y \\
a^{3} \mathrm{D}_{3}^{\circ} \\
{ }^{3} \mathrm{D}_{3}-z^{3} \mathrm{G}_{4}^{\circ} \\
b^{3} \mathrm{~F}_{2}-x^{3} \mathrm{D}_{2}^{2} \\
a^{3} \mathrm{P}_{2}-y^{3} \mathrm{P}_{3}^{0}\end{array}$ & & \\
\hline $\begin{array}{l}2548.685 \\
2546.311 \\
2545.696 \\
2545.460 \\
2544.29\end{array}$ & 2 & $\begin{array}{c}60 \\
5 \\
2 \\
15 \\
10 H\end{array}$ & & $\begin{array}{l}39224.15 \\
39260.72 \\
39270.20 \\
39273.85 \\
39291.90\end{array}$ & $\begin{array}{l}a^{3} \mathrm{P}_{1}-y^{3} \mathrm{P}_{1}^{\circ} \\
a^{3} \mathrm{P}_{1}-y^{3} \mathrm{D}_{2}^{\circ} \\
d^{3} \mathrm{~B}_{3}-v{ }^{3} \mathrm{G}_{4}^{\circ} \\
a^{5} \mathrm{D}_{4}-z^{3} \mathrm{G}_{5}^{\circ} \\
a^{5} \mathrm{P}_{1}-z z^{1} \mathrm{P}_{1}^{0}\end{array}$ & & \\
\hline $\begin{array}{l}2542.935 \\
2542.46 \\
2539.20 \\
2537.619 \\
2536.854\end{array}$ & 2 & $\begin{array}{l}15 \\
20 H l \\
20 H l \\
20 \\
2\end{array}$ & & $\begin{array}{l}39312.84 \\
39320.18 \\
39370.66 \\
39395.19 \\
39407.07\end{array}$ & $\begin{array}{l}b^{3} \mathrm{~F}_{2}-z^{1} \mathrm{P}_{1}^{\circ} \\
a^{1} \mathrm{H}_{5}-1_{4}^{\circ} \\
b{ }^{1} \mathrm{D}_{2}-x^{1} \mathrm{D}_{2}^{\circ} \\
a^{3} \mathrm{G}_{3}-w^{3} \mathrm{D}_{2}^{\circ}\end{array}$ & & \\
\hline $\begin{array}{l}2536.65 \\
2534.519 \\
2534.263 \\
2533.969 \\
2533.365\end{array}$ & $\begin{array}{l}5 \\
1- \\
1\end{array}$ & $\begin{array}{c}2 h \\
80 \\
9 \\
9 \\
15\end{array}$ & & $\begin{array}{l}39410.24 \\
39443.37 \\
39447.35 \\
39451.93 \\
39461.34\end{array}$ & $\begin{array}{l}a{ }^{3} \mathrm{P}_{0}-y{ }^{3} \mathrm{P}_{1}^{\circ} \\
a^{3} \mathrm{H}_{6}-y \\
{ }^{3} \mathrm{H}_{5}^{\circ} \\
a^{3} \mathrm{H}_{5}-y{ }^{3} \mathrm{H}_{4}^{\circ}\end{array}$ & & \\
\hline $\begin{array}{l}2531.902 \\
2531.616 \\
2528.833 \\
2528.466 \\
2527.903\end{array}$ & $\begin{array}{l}10 \\
10 \\
15\end{array}$ & $\begin{array}{l}4+H ? \\
3 \\
220 \\
200 \\
250\end{array}$ & & $\begin{array}{l}39484.14 \\
39488.60 \\
39532.05 \\
39537.79 \\
39546.59\end{array}$ & $\begin{array}{l}a^{3} \mathrm{G}_{5}-y{ }^{1} \mathrm{G}_{4}^{\circ} \\
a^{3} \mathrm{H}_{5}-y \\
a^{3}{ }^{3} \mathrm{H}_{4}-y \\
a^{3} \mathrm{H}_{6}-y^{3} \mathrm{H}_{4}^{\circ} \\
{ }^{3} \mathrm{H}_{6}^{\circ}\end{array}$ & & \\
\hline 2523. 953 & 5 & 100 & & 39608.48 & $\left\{\begin{array}{l}a^{3}{ }^{3} \mathrm{H}_{4}-y{ }^{3} \mathrm{H}_{5}^{\circ} \\
a^{3} \mathrm{P}_{1}-y^{3} \mathrm{P}_{2}^{\circ}\end{array}\right.$ & & \\
\hline $\begin{array}{l}2523.61 \\
2522.513 \\
2522.392 \\
2521.370\end{array}$ & 2 & $\begin{array}{c}1 \\
20 \\
6 \\
4 h\end{array}$ & & $\begin{array}{l}39613.86 \\
39631.09 \\
39632.99 \\
39649.05\end{array}$ & $\begin{array}{l}d^{3} \mathrm{~F}_{4}-v^{3} \mathrm{G}_{5}^{\circ} \\
a^{3} \mathrm{H}_{5}-y^{3} \mathrm{H}_{6}^{\circ} \\
a^{3} \mathrm{~F}_{4}-y^{3} \mathrm{G}_{4}^{\circ}\end{array}$ & & \\
\hline $\begin{array}{l}2520.408 \\
2517.97 \\
2515.722 \\
2514.633 \\
2513.322\end{array}$ & $\begin{array}{l}1 \\
7\end{array}$ & $\begin{array}{c}2 \\
2 h \\
9 \\
200 \\
1\end{array}$ & & $\begin{array}{l}39664.19 \\
39702.59 \\
39738.06 \\
39755.27 \\
39776.00\end{array}$ & $\begin{array}{l}a^{3} \mathrm{~F}_{3}-y^{3} \mathrm{G}_{3}^{\circ} \\
a^{3} \mathrm{~F}_{4}-y^{3} \mathrm{G}_{5}^{\circ}\end{array}$ & & \\
\hline $\begin{array}{l}2512.812 \\
2508.854 \\
2508.26 \\
2506.215 \\
2505.236\end{array}$ & 10 & $\begin{array}{c}2 h \\
4 \\
2 h \\
200 \\
2 h\end{array}$ & & $\begin{array}{l}39784.08 \\
39846.84 \\
39856.27 \\
39888.79 \\
39904.38\end{array}$ & $\begin{array}{l}a^{3} \mathrm{H}_{4}-x^{3} \mathrm{~F}_{3}^{0} \\
a^{5} \mathrm{D}_{4}-z^{3} \mathrm{~F}_{3}^{0} \\
a^{3} \mathrm{~F}_{3}-y^{3} \mathrm{G}_{4}^{8}\end{array}$ & & \\
\hline $\begin{array}{l}2504.94 \\
2504.290 \\
2503.018 \\
2500.076 \\
2497.002\end{array}$ & 9 & $\begin{array}{c}2 h \\
7 h \\
180 \\
4 \\
4\end{array}$ & & $\begin{array}{l}39909.09 \\
39919.45 \\
39939.74 \\
39986.73 \\
40035.96\end{array}$ & $\begin{array}{l}c^{3} \mathrm{~F}_{4}-v^{3} \mathrm{G}_{5}^{\circ} \\
a^{3} \mathrm{~F}_{2}-y^{3} \mathrm{G}_{3}^{\circ} \\
a^{5} \mathrm{D}_{3}-z^{3} \mathrm{~F}_{3}^{0} \\
a^{3} \mathrm{H}_{5}-x^{3} \mathrm{~F}_{4}^{\circ}\end{array}$ & (0) 0.76 & (0) $0.71 \mathrm{~s}$ \\
\hline $\begin{array}{l}2494.721 \\
2494.562 \\
2493.576 \\
2488.616 \\
2485.490\end{array}$ & $\begin{array}{l}2 h \\
2 h \\
3 \\
1-\end{array}$ & $\begin{array}{c}4 h \\
3 h \\
15 \\
6 \\
3\end{array}$ & & $\begin{array}{l}40072.56 \\
40075.11 \\
40090.96 \\
40170.86 \\
40221.37\end{array}$ & $\begin{array}{l}a^{5} \mathrm{D}_{4}-z^{3} \mathrm{~F}_{4}^{\circ} \\
a^{3} \mathrm{~F}_{4}-y^{3} \mathrm{~F}_{4}^{0} \\
a^{5} \mathrm{D}_{3}-z^{3} \mathrm{~F}_{4}^{0}\end{array}$ & & \\
\hline $\begin{array}{l}2483.064 \\
2482.307 \\
2479.518 \text { l } \\
2479.043 \\
2478.621\end{array}$ & $\begin{array}{l}4 \\
5 \\
7 \\
8 \\
2\end{array}$ & $\begin{array}{r}120 \\
150 \\
180 \\
200 \\
20\end{array}$ & & $\begin{array}{l}40260.67 \\
40272.94 \\
40318.24 \\
40325.96 \\
40332.83\end{array}$ & $\begin{array}{l}b^{3}{ }^{3} \mathrm{~F}_{2}-w^{3} \mathrm{D}_{1}^{\circ} \\
a^{3} \mathrm{P}_{2}-z^{3} \mathrm{Si}_{1}^{1} \\
b^{3} \mathrm{~F}_{4}-w^{3} \mathrm{D}_{3}^{3} \\
b^{3} \mathrm{~F}_{3}-w^{3} \mathrm{D}_{2}^{\circ} \\
a^{3} \mathrm{H}_{4}-z^{3} \mathrm{I}_{5}^{\circ}\end{array}$ & (0) 0.93 & (0) $0.92 \mathrm{~b}$ \\
\hline
\end{tabular}

See footnotes at end of table. 
TABLE 1.-Second spectrum of Vanadium-Continued

\begin{tabular}{|c|c|c|c|c|c|c|c|}
\hline \multirow{3}{*}{$\lambda_{\mathrm{sir}} \mathrm{A}$} & \multicolumn{2}{|c|}{2} & 3 & 4 & 5 & & \\
\hline & & nsity & \multirow{2}{*}{$\begin{array}{l}\text { Temp. } \\
\text { class }\end{array}$} & \multirow{2}{*}{$\begin{array}{l}\text { Wave } \\
\text { No. vac }\end{array}$} & \multirow{2}{*}{$\begin{array}{c}\text { Term } \\
\text { combination }\end{array}$} & \multicolumn{2}{|c|}{ Zeeman effect } \\
\hline & Arc & Spark & & & & Observed & Computed \\
\hline $\begin{array}{l}2478.340 \\
2476.963 \\
2476.295 \\
2475.865 \\
2475.451\end{array}$ & $\begin{array}{l}2 \\
2\end{array}$ & $\begin{array}{c}1 \\
1 \\
5 h \\
30 \\
20\end{array}$ & & $\begin{array}{l}40337.40 \\
40359.82 \\
40370.71 \\
40377.72 \\
40384.48\end{array}$ & $\begin{array}{l}a^{3} \mathrm{P}_{2}-x{ }^{3} \mathrm{~F}_{2}^{\circ} \\
a^{3} \mathrm{~F}_{3}-y^{3} \mathrm{~F}_{2}^{\circ} \\
a^{3} \mathrm{H}_{6}-z z^{3} \mathrm{I}_{6}^{\circ} \\
b^{3} \mathrm{~F}_{2}-w^{3} \mathrm{D}_{2}^{2} \\
b^{3} \mathrm{~F}_{3}-w^{3} \mathrm{D}_{3}^{\circ}\end{array}$ & & \\
\hline $\begin{array}{l}2472.870 \\
2471.119 \\
2469.388 \\
2468.654 \\
2465.270\end{array}$ & $\begin{array}{l}2 \\
1 \\
5\end{array}$ & $\begin{array}{r}5 \\
25 \\
5 \\
8 \\
150\end{array}$ & & $\begin{array}{l}40426.62 \\
40455.27 \\
40483.62 \\
40495.66 \\
40551.24\end{array}$ & $\begin{array}{c}a^{3} \mathrm{~F}_{3}-y^{3} \mathrm{~F}_{4}^{\circ} \\
a^{3} \mathrm{H}_{5}-z^{3} \mathrm{I}_{6}^{\circ} \\
a^{3} \mathrm{P}_{2}-x^{3} \mathrm{~F}_{3}^{\circ} \\
a^{3} \mathrm{~F}_{4}-z^{1} \mathrm{H}_{5}^{\circ} \\
a^{3} \mathrm{G}_{5}-x^{3} \mathrm{G}_{5}^{\circ}\end{array}$ & & \\
\hline $\begin{array}{l}2464.65 \\
2464.094 \\
2463.157 \\
2461.495 \\
2460.73\end{array}$ & $\begin{array}{l}1 \\
3\end{array}$ & $\begin{array}{r}1 \\
15 \\
3 \\
40 \\
1\end{array}$ & & $\begin{array}{l}40561.44 \\
40570.59 \\
40586.03 \\
40613.43 \\
40626.05\end{array}$ & $\begin{array}{c}a^{3} \mathrm{~F}_{2}-y^{3} \mathrm{~S}_{2}^{\circ} \\
a^{3} \mathrm{~F}_{2}-y^{3} \mathrm{~F}_{3}^{0} \\
a^{3} G_{4}-y y^{1} \mathrm{~F}_{3}^{0} \\
a^{3} \mathrm{H}_{6}-z^{3} \mathrm{I}_{7}^{\circ} \\
a^{3} \mathrm{~F}_{4}-z^{1}{ }^{1} \mathrm{G}_{4}\end{array}$ & & \\
\hline $\begin{array}{l}2459.358 \\
2459.233 \\
2458.288 \\
2457.803 \\
2457.446\end{array}$ & $\begin{array}{l}1 \\
3 \\
1\end{array}$ & $\begin{array}{r}15 \\
5 \\
50 \\
5 \\
30\end{array}$ & & $\begin{array}{l}40648.71 \\
40650.77 \\
40666.40 \\
40674.43 \\
40680.34\end{array}$ & $\begin{array}{l}a^{3} \mathrm{G}_{5}-x^{3} \mathrm{G}_{4}^{\circ} \\
a^{3} \mathrm{G}_{4}-x^{3} \mathrm{G}_{5}^{\circ} \\
a^{3} \mathrm{P}_{1}-z^{3} \mathrm{~S}_{1}^{\circ} \\
a^{3} \mathrm{G}_{3}-y^{1} \mathrm{~F}_{3}^{\circ}\end{array}$ & & \\
\hline $\begin{array}{l}2456.508 \\
2454.654 \\
2453.857 \\
2453.346 \\
2452.773\end{array}$ & 4 & $\begin{array}{r}3 \\
2 \\
3 \\
80 \\
4\end{array}$ & & $\begin{array}{l}40695.87 \\
40726.60 \\
40739.83 \\
40748.32 \\
40757.84\end{array}$ & $\begin{array}{l}a^{3} \mathrm{P}_{2}-x^{3} \mathrm{D}^{\circ} \\
a^{3} \mathrm{~F}_{3}-z^{1} \mathrm{~F}_{3}^{\circ} \\
a^{1} \mathrm{H}_{5}-w^{3} \mathrm{G}_{4} \\
a^{3} \mathrm{G}_{4}-x^{3} \mathrm{G}_{4}^{\circ}\end{array}$ & & \\
\hline $\begin{array}{l}2450.734 \\
2450.619 \\
2450.236 \\
2448.46 \\
2447.608\end{array}$ & $\begin{array}{l}1- \\
1- \\
1\end{array}$ & $\begin{array}{c}20 \\
9 \\
10 h \\
5 H \\
20\end{array}$ & & $\begin{array}{l}40791.74 \\
40793.65 \\
40800.03 \\
40829.63 \\
40843.84\end{array}$ & $\begin{array}{l}a{ }^{3} \mathrm{P}_{2}-x^{3} \mathrm{D}_{2}^{\circ} \\
a^{3} \mathrm{G}_{4}-x^{3} \mathrm{G}_{3}^{2} \\
a^{3} \mathrm{G}_{5}-y^{1} \mathrm{H}_{3}^{\circ}\end{array}$ & & \\
\hline $\begin{array}{l}2446.697 \\
2445.336 \\
2445.107 \\
2444.967 \\
2441.664\end{array}$ & 2 & $\begin{array}{c}30 \\
4 \\
6 \\
60 \\
4 h\end{array}$ & & $\begin{array}{l}40859.04 \\
40881.78 \\
40885.61 \\
40887.95 \\
40943.26\end{array}$ & $\begin{array}{l}a{ }^{3} \mathrm{P}_{2}-x^{3} \mathrm{D}_{3}^{\circ} \\
a^{3} \mathrm{~F}_{3}-z^{1} \mathrm{G}_{4}^{9} \\
a^{3} \mathrm{P}_{0}-z^{3} \mathrm{~S}_{1}^{\circ} \\
a^{3} \mathrm{G}_{3}-x^{3} \mathrm{G}_{3}^{3} \\
a^{3} \mathrm{G}_{4}-y^{1} \mathrm{H}_{5}^{\circ}\end{array}$ & & \\
\hline $\begin{array}{l}2439.774 \\
2438.039 \\
2436.570 \\
2434.94 \\
2432.976\end{array}$ & 2 & $\begin{array}{c}4 h \\
10 h \\
5 H \\
5 ? \\
20\end{array}$ & & $\begin{array}{l}40974.97 \\
41004.13 \\
41028.85 \\
41056.31 \\
41089.46\end{array}$ & $\begin{array}{l}a{ }^{3} \mathrm{~F}_{3}-z^{1} \mathrm{D}_{2}^{\circ} \\
a^{3} \mathrm{P}_{1}-x^{3} \mathrm{D}_{1}^{\circ}\end{array}$ & & \\
\hline $\begin{array}{l}2431.59 \\
2427.316 \\
2423.030 \\
2408.430 \\
2407.592\end{array}$ & $\begin{array}{l}1 \\
2\end{array}$ & $\begin{array}{r}4 \\
20 \\
6 \\
15 \\
5\end{array}$ & & $\begin{array}{l}41112.87 \\
41185.26 \\
41258.10 \\
41508.19 \\
41522.64\end{array}$ & $\begin{array}{c}b^{1} \mathrm{D}_{2}-x^{1} \mathrm{~F}_{3}^{\circ} \\
a^{3} \mathrm{P}_{1}-x^{3} \mathrm{D}_{2}^{\circ} \\
a^{3} \mathrm{~F}_{2}-z^{1} \mathrm{D}_{2}^{0} \\
a^{3} \mathrm{P}_{0}-z^{1} \mathrm{P}_{1}^{0} \\
a^{3} \mathrm{H}_{5}-y^{1} \mathrm{G}_{4}^{\circ}\end{array}$ & & \\
\hline $\begin{array}{l}2406.989 \\
2405.817 \\
2403.240 \\
2400.892 \\
2400.166\end{array}$ & $\begin{array}{l}1 \\
1\end{array}$ & $\begin{array}{c}5 \\
2 \\
9 \\
40 h \\
4\end{array}$ & & $\begin{array}{l}41533.04 \\
41553.27 \\
41597.82 \\
41638.50 \\
41651.09\end{array}$ & 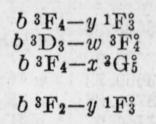 & & \\
\hline $\begin{array}{l}2397.622 \\
2396.927 \\
2394.92 \\
2393.814 \\
2392.698\end{array}$ & 1 & $\begin{array}{l}6 \\
4 H \\
2 h ? \\
8 \\
8 h\end{array}$ & & $\begin{array}{l}41695.28 \\
41707.37 \\
41742.32 \\
41761.61 \\
41781.08\end{array}$ & $\begin{array}{l}b^{3} \mathrm{~F}_{4}-x^{3} \mathrm{G}_{4}^{\circ} \\
c^{3} \mathrm{P}_{2}-w^{3} \mathrm{~F}_{2}^{0} \\
b^{3} \mathrm{~F}_{3}-x{ }^{3} \mathrm{G}_{4}^{0}\end{array}$ & & \\
\hline $\begin{array}{l}2392.17 \\
2391.226 * \\
2390.470 \\
2389.696 \\
2389.144\end{array}$ & 10 & $\begin{array}{c}2 h \\
10 h \\
15 h \\
100 \\
2\end{array}$ & & $\begin{array}{l}41790.30 \\
41806.80 \\
41820.02 \\
41833.56 \\
41843.23\end{array}$ & $\begin{array}{l}c^{3} \mathrm{P}_{2}-w^{3} \mathrm{~F}_{3}^{\circ} \\
b^{3} \mathrm{~F}_{3}-x^{3} \mathrm{G}_{3}^{\circ} \\
a^{3} \mathrm{~F}_{2}-y{ }^{3} \mathrm{D}_{1}^{\circ} \\
a^{3} \mathrm{P}_{2}-w^{3} \mathrm{D}_{1}^{\circ}\end{array}$ & & \\
\hline $\begin{array}{l}2388.260 \\
2387.93 \\
2386.45 \\
2384.996 \\
2383.995\end{array}$ & 10 & $\begin{array}{l}5 \\
3 h \\
2 \\
6 h \\
80\end{array}$ & & $\begin{array}{l}41858.72 \\
41864.50 \\
41890.46 \\
41916.00 \\
41933.60\end{array}$ & $\begin{array}{l}b{ }^{3} \mathrm{~F}_{2}-x^{3} \mathrm{G}_{3}^{\circ} \\
b{ }^{3} \mathrm{~F}_{4}-y{ }^{1} \mathrm{H}_{5}^{\circ} \\
a^{3} \mathrm{~F}_{3}-y^{3} \mathrm{D}_{2}^{\circ}\end{array}$ & (0) 0.96 & $\begin{array}{l}(0) 1.28 \mathrm{~s} \\
(0) 0.97 \mathrm{~b}\end{array}$ \\
\hline
\end{tabular}

See footnotes at end of table. $235535-40-8$ 
TABLE 1.-Second spectrum of Vanadium-Continued

\begin{tabular}{|c|c|c|c|c|c|c|c|}
\hline \multirow{3}{*}{$\lambda_{\text {air }} \mathrm{A}$} & \multicolumn{2}{|c|}{2} & 3 & 4 & 5 & \multicolumn{2}{|c|}{6} \\
\hline & \multicolumn{2}{|c|}{ Intensity } & \multirow{2}{*}{$\begin{array}{c}\text { Temp. } \\
\text { class }\end{array}$} & \multirow{2}{*}{$\begin{array}{l}\text { Wave } \\
\text { No. vac }\end{array}$} & \multirow{2}{*}{$\begin{array}{c}\text { Term } \\
\text { combination }\end{array}$} & \multicolumn{2}{|c|}{ Zeeman effect } \\
\hline & Arc & Spark & & & & Observed & Computed \\
\hline $\begin{array}{l}2383.432 \\
2382.032 \\
2380.910 \\
2379.149 \\
2376.33\end{array}$ & $\begin{array}{r}8 \\
12 \\
10\end{array}$ & $\begin{array}{c}6 H \\
60+\mathrm{Fc} ? \\
120 \\
100 \\
1\end{array}$ & & $\begin{array}{l}41943.50 \\
41968.15 \\
41987.92 \\
42019.00 \\
42068.84\end{array}$ & $\begin{array}{l}a^{3} \mathrm{~F}_{4}-y{ }^{3} \mathrm{D}_{3}^{\circ} \\
a^{3} \mathrm{P}_{2}-w^{3} \mathrm{D}_{3} \\
a^{5} \mathrm{P}_{2}-y^{3} \mathrm{~S}^{3}\end{array}$ & & \\
\hline $\begin{array}{l}2374.649 \\
2374.31 \\
2372.584 \\
2372.168 \\
2366.883\end{array}$ & $\begin{array}{l}1 \\
4 \\
3\end{array}$ & $\begin{array}{l}\quad 6 \\
2 H ? \\
20 \\
15 H \\
20\end{array}$ & & $\begin{array}{l}42098.62 \\
42104.63 \\
42135.26 \\
42142.65 \\
42236.73\end{array}$ & $\begin{array}{l}a^{3} \mathrm{~B}_{2}-y{ }^{3} \mathrm{P}_{1}^{\circ} \\
a^{1} \mathrm{~F}_{3}-u{ }^{3} \mathrm{D}_{3}^{\circ} \\
a^{3} \mathrm{~F}_{2}-y{ }^{3} \mathrm{D}_{2}^{\circ} \\
a^{3} \mathrm{P}_{1}-w^{3}{ }^{3} \mathrm{D}_{1}^{\circ}\end{array}$ & & \\
\hline $\begin{array}{l}2366.490 \\
2364.390 \\
2362.632 \\
2360.334 \\
2357.810\end{array}$ & $\begin{array}{l}4 \\
2 \\
5 \\
6\end{array}$ & $\begin{array}{r}25 \\
2 \\
20 \\
50 \\
60\end{array}$ & & $\begin{array}{l}42243.75 \\
42281.27 \\
42312.73 \\
42353.92 \\
42399.25\end{array}$ & $\begin{array}{l}a^{3}{ }^{3} \mathrm{~F}_{3}-y \\
a^{3}{ }^{3} \mathrm{D}_{3}^{\circ} \\
a^{3} \mathrm{~F}_{3}-y{ }^{3} \mathrm{P}_{2}^{\circ}-x \\
a^{1} \mathrm{P}_{2}^{\circ} \\
a^{3} \mathrm{P}_{1}-w^{3}{ }^{3} \mathrm{D}_{2}^{\circ} \\
a^{1} \mathrm{H}_{5}-x^{1} \mathrm{G}_{4}^{\circ}\end{array}$ & (0) $1.30 \mathrm{nw}_{2}$ & (0) $1.24 \mathrm{~b}$ \\
\hline $\begin{array}{l}2357.532 \\
2356.209 \\
2355.232 \\
2354.656 \\
2353.16\end{array}$ & 2 & $\begin{array}{l}3 H \\
3 H \\
4 \\
20 \\
1 ?\end{array}$ & & $\begin{array}{l}42404.25 \\
42428.06 \\
42445.66 \\
42456.04 \\
42483.03\end{array}$ & $\begin{array}{l}a^{3}{ }^{3} \mathrm{~F}_{2}-y \\
a^{3} \mathrm{~B}_{0} \mathrm{D}_{3}^{0} \\
a^{3} \mathrm{P}_{2}-x^{3} \mathrm{D}^{3} \mathrm{D}_{2}^{\circ} \mathrm{i}\end{array}$ & & \\
\hline $\begin{array}{l}2352.177 \\
2351.24 \\
2347.507 \\
2346.868 \\
2342.142\end{array}$ & $\begin{array}{r}10 \\
1 \\
5 \\
10\end{array}$ & $\begin{array}{l}100 \\
6 H \\
8 \\
25 \\
60+h\end{array}$ & & $\begin{array}{l}42500.78 \\
42517.72 \\
42585.31 \\
42596.92 \\
42682.86\end{array}$ & $\begin{array}{l}a^{3} \mathrm{H}_{8}-x^{3} \mathrm{G}_{5}^{\circ} \\
a^{3}{ }^{3} \mathrm{H}_{5}-x^{3} \mathrm{G}_{5}^{\circ} \\
a^{3} \mathrm{H}_{4}-y{ }^{1} \mathrm{~F}_{3}^{0} \\
a^{3} \mathrm{H}_{5}-x{ }^{3} \mathrm{G}_{4}^{0}\end{array}$ & (0) 1.03 & (0) $1.02 b$ \\
\hline $\begin{array}{l}2341.358 \\
2337.956 \\
2336.098 \\
2335.480 \\
2335.326\end{array}$ & $\begin{array}{l}4 \\
6 \\
2\end{array}$ & $\begin{array}{r}4 \\
4 \\
30 \\
40 \\
10\end{array}$ & & $\begin{array}{l}42697.15 \\
42759.27 \\
42793.28 \\
42804.60 \\
42807.42\end{array}$ & $\begin{array}{l}a^{3} \mathrm{H}_{6}-z^{1} \mathrm{I}_{6}^{\circ} \\
a^{3} \mathrm{H}_{4}-x^{3} \mathrm{G}_{4}^{\circ} \\
a^{3} \mathrm{H}_{6}-y^{1} \mathrm{H}_{5}^{\circ} \\
a^{3} \mathrm{H}_{4}-x^{3} \mathrm{G}_{3}^{0} \\
a^{3} \mathrm{P}_{2}-x^{3} \mathrm{P}_{2}^{0}\end{array}$ & & \\
\hline $\begin{array}{l}2335.204 \\
2330.144 \\
2321.855 \\
2314.99 \\
2314.055\end{array}$ & 1 & $\begin{array}{l}2 \\
12 \\
1 \\
2 h ? \\
3\end{array}$ & & $\begin{array}{l}42899.66 \\
42902.62 \\
43055.76 \\
43183.44 \\
43200.88\end{array}$ & $\begin{array}{l}a^{3} \mathrm{P}_{2}-x{ }^{3} \mathrm{P}_{\mathrm{i}}^{0} \\
a^{3} \mathrm{~F}_{4}-y^{3} \mathrm{H}_{5}^{0} \\
a^{3} \mathrm{P}_{1}-x{ }^{3} \mathrm{P}_{2}^{0}\end{array}$ & & \\
\hline $\begin{array}{l}2313.939 \\
2312.299 \\
2311.91 \\
2309.072 \\
2308.831\end{array}$ & 1 & $\begin{array}{c}9 \\
2 \\
1 ? \\
10 \\
5\end{array}$ & & $\begin{array}{l}43203.04 \\
43233.68 \\
43240.96 \\
43294.10 \\
43298.62\end{array}$ & $\begin{array}{l}a^{3} \mathrm{P}_{1}-x^{3} \mathrm{P}_{1}^{\circ} \\
a^{3} \mathrm{P}_{2}-y^{1} \mathrm{~F}_{3}^{3} \\
a^{3} \mathrm{~F}_{3}-y^{3} \mathrm{H}_{4}^{\circ} \\
a^{3} \mathrm{~F}_{4}-x^{3} \mathrm{~F}_{3}^{0} \\
a^{3} \mathrm{P}_{1}-x^{3} \mathrm{P}_{0}^{0}\end{array}$ & & \\
\hline $\begin{array}{l}2304.785 \\
2303.238 \\
2302.256 \\
2295.504 \\
2294.992\end{array}$ & $\begin{array}{l}4 \\
7\end{array}$ & $\begin{array}{c}7 h \\
10 \\
5 \\
20 \\
40\end{array}$ & & $\begin{array}{l}43374.62 \\
43403.75 \\
43422.26 \\
43549.97 \\
43559.68\end{array}$ & $\begin{array}{l}a^{3} \mathrm{~F}_{3}-x^{3} \mathrm{~F}_{2}^{0} \\
a^{3} \mathrm{P}_{0}-x^{3} \mathrm{P}_{1}^{0} \\
a^{3} \mathrm{~F}_{3}-x^{3} \mathrm{~F}_{3}^{0} \\
a^{3} \mathrm{~F}_{4}-x^{3} \mathrm{~F}_{4}^{0}\end{array}$ & & \\
\hline 2292.588 & 5 & 30 & & 43605. 36 & $\left\{\begin{array}{l}a^{3} \mathrm{~F}_{2}-x^{3} \mathrm{~F}_{2}^{2} \\
a^{1} \mathrm{D}_{2}-x\end{array}\right.$ & & \\
\hline $\begin{array}{l}2291.381 \\
2289.219 \\
2284.920 \\
2284.748\end{array}$ & $\begin{array}{l}4 \\
1\end{array}$ & $\begin{array}{l}3 h \\
70 \\
15 \\
10\end{array}$ & & $\begin{array}{l}43628.32 \\
43669.53 \\
43751.68 \\
43754.97\end{array}$ & $\begin{array}{l}a{ }^{3} \mathrm{~F}_{4}-x{ }^{3} \mathrm{D}_{3}^{\circ} \\
a^{3} \mathrm{~F}_{2}-x \\
a^{3} \mathrm{~B}_{3}^{\circ} \mathrm{P}_{2}-y^{3} \mathrm{~S}_{1}^{\circ}\end{array}$ & & \\
\hline $\begin{array}{l}2283.766 \\
2283.469 \\
2282.863\end{array}$ & & $\begin{array}{r}40 \\
7 \\
6\end{array}$ & & $\begin{array}{l}43773.79 \\
43779.48 \\
43791.10\end{array}$ & $\begin{array}{l}a^{3} \mathrm{D}_{3}-w^{3} \mathrm{~F}_{3}^{\circ} \\
a^{3} \mathrm{D}_{2}-w^{3} \mathrm{~F}_{2}^{2}\end{array}$ & & \\
\hline 2281.601 & 2 & 60 & & 43815.32 & $\begin{array}{l}a^{3} \mathrm{~F}_{3}-x^{3} \mathrm{~F}_{4}^{0} \\
a^{3} \mathrm{D}_{1}-w^{3} \mathrm{~F}_{2}^{2} \\
b^{3} \mathrm{H}_{1}-w^{3} \mathrm{G}^{3}\end{array}$ & & \\
\hline 2281.235 & 2 & 60 & & 43822.35 & $a^{3} \mathrm{D}_{3}-w^{3} \mathrm{w}^{3} \mathrm{~F}_{4}^{3}$ & & \\
\hline $\begin{array}{l}2280.581 \\
2280.338 \\
2279.762 \\
2279.376 \\
2278.972\end{array}$ & $\begin{array}{l}1 \\
1\end{array}$ & $\begin{array}{r}4 \\
60 \\
20 \\
15 \\
40\end{array}$ & & $\begin{array}{l}43834.91 \\
43839.58 \\
43850.66 \\
43858.08 \\
43865.86\end{array}$ & $\begin{array}{c}a^{3} \mathrm{D}_{2}-w^{3} \mathrm{~F}_{3}^{0} \\
b^{3} \mathrm{H}_{5}-w^{3} \mathrm{G}_{4}^{0} \\
a^{3} \mathrm{~F}_{3}-x^{3} \mathrm{D}_{2}^{8} \\
b^{3} \mathrm{H}_{6}-w^{3} \mathrm{G}_{5}^{\circ}\end{array}$ & & \\
\hline
\end{tabular}

See footnotes at end of table. 
TABLE 1.-Second spectrum of Vanadium-Continued

\begin{tabular}{|c|c|c|c|c|c|c|c|}
\hline \multirow{3}{*}{$\lambda_{\mathrm{B} \text { ir }} \mathrm{A}$} & \multicolumn{2}{|c|}{2} & 3 & 4 & 5 & & \\
\hline & & nsity & \multirow{2}{*}{$\begin{array}{c}\text { Temp. } \\
\text { class }\end{array}$} & \multirow{2}{*}{$\begin{array}{l}\text { Wave } \\
\text { No. vac }\end{array}$} & \multirow{2}{*}{$\begin{array}{c}\text { Term } \\
\text { combination }\end{array}$} & \multicolumn{2}{|c|}{ Zeeman effect } \\
\hline & Arc & Spark & & & & Observed & Computed \\
\hline $\begin{array}{l}2278.098 \\
2277.792 \\
2275.883 \\
2275.586 \\
2274.658\end{array}$ & & $\begin{array}{l}4 h \\
1 \\
7 \\
7 \\
1\end{array}$ & & $\begin{array}{l}43882.69 \\
43888.58 \\
43925.39 \\
43931.12 \\
43949.04\end{array}$ & $\begin{array}{l}b^{3} \mathrm{H}_{4}-w^{3} \mathrm{G}_{4}^{\circ} \\
a^{3} \mathrm{~F}_{3}-x^{3} \mathrm{D}_{3}^{0} \\
a^{3} \mathrm{~F}_{3}-z^{3} \mathrm{P}_{2}^{0} \\
b^{3} \mathrm{H}_{5}-w^{3} \mathrm{G}_{5}^{\circ}\end{array}$ & & \\
\hline $\begin{array}{l}2273.89 \\
2273.616\end{array}$ & & $\begin{array}{l}2 \\
9\end{array}$ & & $\begin{array}{l}43963.88 \\
43969.18\end{array}$ & $\begin{array}{l}a^{3}{ }^{3} \mathrm{~F}_{2}-x \\
b^{3}{ }^{3} \mathrm{D}_{2}-x_{1}^{\circ} \\
{ }^{1} \mathrm{D}_{2}^{8}\end{array}$ & & \\
\hline 2273.024 & & $40 h$ & & 43980.63 & $\left\{\begin{array}{c}a^{5}{ }^{5} \mathrm{~F}_{1}-z{ }^{3} \mathrm{P}_{0}^{0} \\
b{ }^{3} \mathrm{D}_{3}-{ }^{3} \mathrm{D}^{3}\end{array}\right.$ & & \\
\hline $\begin{array}{l}2272.437 \\
2271.848\end{array}$ & 1 & $\begin{array}{r}1 \\
10\end{array}$ & & $\begin{array}{l}43991.99 \\
44003.40\end{array}$ & $\begin{array}{l}d^{3} \mathrm{~F}_{2}-w \\
a^{5} \mathrm{~F}_{2}-z^{3} \mathrm{~F}_{3}^{3} \\
{ }^{3} \mathrm{P}_{1}^{0}\end{array}$ & & \\
\hline $\begin{array}{l}2271.185 \\
2269.293 \\
2268.55 \\
2268.361 \\
2267.712\end{array}$ & & $\begin{array}{l}8 \\
3 \\
1 \\
4 \\
3\end{array}$ & & $\begin{array}{l}44016.24 \\
44052.94 \\
44067.36 \\
44071.03 \\
44083.65\end{array}$ & $\begin{array}{l}a^{5} \mathrm{5}_{2}-z^{3} \mathrm{P}_{2}^{0} \\
a^{5} \mathrm{~F}_{2}-z^{5} \mathrm{P}_{1}^{0} \\
a^{5} \mathrm{~F}_{3}-z^{5} \mathrm{P}_{2}^{0} \\
a^{5} \mathrm{~F}_{4}-z^{5} \mathrm{P}_{3}^{0}\end{array}$ & & \\
\hline $\begin{array}{l}2267.612 \\
2263.612 \\
2262.70 \\
2262.404 \\
2261.850\end{array}$ & & $\begin{array}{c}5 \\
3 \\
2 \\
9 h \\
10 h\end{array}$ & & $\begin{array}{l}44085.59 \\
44163.48 \\
44181.28 \\
44187.06 \\
44197.89\end{array}$ & $\begin{array}{l}a^{5}{ }^{5} \mathrm{~F}_{1}-z{ }^{3} \mathrm{P}_{1}^{\circ} \\
a{ }^{3} \mathrm{~F}_{2}-z{ }^{1} \mathrm{P}_{1}^{\circ} \\
b^{3} \mathrm{D}_{3}-v^{3} \mathrm{D}_{2}^{0} \\
b^{3} \mathrm{D}_{2}-v^{3} \mathrm{D}_{2}^{\circ}\end{array}$ & & \\
\hline $\begin{array}{l}2261.084 \\
2258.814 \\
2258.64 \\
2256.984 \\
2255.95\end{array}$ & ? & $\begin{array}{l}30 \\
50+\mathrm{I} \\
2 \\
20+\mathrm{I} ? \\
1 ?\end{array}$ & & $\begin{array}{l}44212.85 \\
44257.28 \\
44260.69 \\
44293.17 \\
44313.46\end{array}$ & $\begin{array}{l}a^{5}{ }^{5} \mathrm{~F}_{4}-y{ }^{5} \mathrm{D}_{3}^{0} \\
a^{5} \mathrm{~F}_{5}-y \\
c^{3} \mathrm{~S}^{5} \mathrm{D}_{4}^{0} \\
a^{5} \mathrm{P}_{2}-v^{3} \mathrm{D}_{3}^{3} \\
b^{5} \mathrm{~F}_{3}-y^{5} \mathrm{D}_{2}^{2} \\
{ }^{3} \mathrm{D}_{2}-v^{3} \mathrm{D}_{1}^{1}\end{array}$ & & \\
\hline $\begin{array}{l}2252.953 \\
2251.550 \\
2251.114 \\
2250.800 \\
2250.490\end{array}$ & $1-$ & $\begin{array}{l}7 \\
7 \\
6 \\
5 \\
5\end{array}$ & & $\begin{array}{l}44372.41 \\
44400.05 \\
44408.65 \\
44414.85 \\
44420.96\end{array}$ & $\begin{array}{l}a^{5} \mathrm{~F}_{3}-y^{5} \mathrm{D}_{3}^{2} \\
b^{3} \mathrm{D}_{1}-v^{3} \mathrm{D}_{1}^{\circ} \\
a^{5} \mathrm{~F}_{2}-y^{5} \mathrm{D}_{2}^{2} \\
a^{5} \mathrm{~F}_{2}-y^{5} \mathrm{D}_{1}^{\circ}\end{array}$ & & \\
\hline $\begin{array}{l}2250.382 \\
2248.913 \\
2248.45\end{array}$ & & $\begin{array}{l}3 \\
4 \\
1\end{array}$ & & $\begin{array}{l}44423.10 \\
44452.11 \\
44461.26\end{array}$ & $\begin{array}{l}a{ }^{5} \mathrm{~F}_{1}-y{ }^{5} \mathrm{D}_{0}^{0} \\
a^{5} \mathrm{~F}_{4}-y \\
c^{3} \mathrm{~S}_{2} \mathrm{D}_{4}^{8} \\
{ }^{3} \mathrm{P}_{2}^{0}\end{array}$ & & \\
\hline 2246.65 & & $1 ?$ & & 44496.88 & $\left\{\begin{array}{l}a^{a}{ }^{5} \mathrm{~F}_{1}-y \\
c^{3} \mathrm{P}_{1}-x \mathrm{D}_{2}^{2} \\
c^{2} \mathrm{D}_{2}^{8}\end{array}\right.$ & & \\
\hline 2246.332 & & 3 & & 44503.18 & $a \mathrm{~F}_{1}-y{ }^{5} \mathrm{D}_{\mathrm{i}}$ & & \\
\hline $\begin{array}{l}2243.468 \\
2229.985 \\
2224.845\end{array}$ & 3 & $\begin{array}{c}4 h \\
80 \\
1\end{array}$ & & $\begin{array}{l}44559.98 \\
44829.37 \\
44932.93\end{array}$ & $a^{3} \mathrm{~F}_{4}-w^{3} \mathrm{D}_{3}^{\circ}$ & & \\
\hline $\begin{array}{l}2220.214 \\
2219.408\end{array}$ & 3 & $\begin{array}{r}1 \\
100 \\
3\end{array}$ & & $\begin{array}{l}44932.93 \\
45026.65 \\
45043.00\end{array}$ & $\begin{array}{l}a^{3} \mathrm{~F}_{3}-w{ }^{3} \mathrm{D}_{2}^{2} \\
d^{3} \mathrm{~F}_{2}-t{ }^{3} \mathrm{D}_{1}^{\circ} ?\end{array}$ & & \\
\hline 2217. 32 & & 8 & & 45085.41 & $\left\{\begin{array}{l}a^{2}{ }^{3} \mathrm{~F}_{3}-w w^{3} \mathrm{D}_{3}^{0} \\
d^{3} \mathrm{~F}_{2}-t^{3} \mathrm{D}_{2}^{2}\end{array}\right.$ & & \\
\hline $\begin{array}{l}2215.786 \\
2211.38 \\
2211.16 \\
2210.305\end{array}$ & $1-$ & $\begin{array}{l}9 h \\
2 h ? \\
2 \\
8\end{array}$ & & $\begin{array}{l}45116.62 \\
45206.50 \\
45211.00 \\
45228.48\end{array}$ & $\begin{array}{l}d^{3} \mathrm{~F}_{3}-t^{3} \mathrm{D}_{3}^{0} \\
a^{3} \mathrm{~B}_{2}-w{ }^{3} \mathrm{D}_{2}^{\circ}\end{array}$ & & \\
\hline $\begin{array}{l}2210.029 \\
2205.70 \\
2199.660 \\
2199.443 \\
2198.524\end{array}$ & 2 & $\begin{array}{r}10 \\
2 \\
7 \\
10 \\
20\end{array}$ & & $\begin{array}{l}45234.13 \\
45322.90 \\
45447.34 \\
45451.82 \\
45470.82\end{array}$ & $\begin{array}{c}d^{3} \mathrm{~B}_{4}-t^{3} \mathrm{D}_{3}^{\circ} \\
a^{1} \mathrm{D}_{2}-x x^{1} \mathrm{~F}_{3}^{0} \\
c^{3} \mathrm{~F}_{2}-t^{3} \mathrm{D}_{1}^{\circ} ? \\
c^{3} \mathrm{~F}_{3}-t^{3} \mathrm{D}_{2}^{\circ} \\
b^{3} \mathrm{P}_{2}-v^{3} \mathrm{D}_{3}^{3}\end{array}$ & & \\
\hline $\begin{array}{l}2195.69 \\
2195.10 \\
2194.84 \\
2193.34 \\
2192.91\end{array}$ & & $\begin{array}{r}15 \\
2 \\
8 \\
2 \\
2 h\end{array}$ & & $\begin{array}{l}45529.50 \\
45541.74 \\
45547.13 \\
45578.27 \\
45587.21\end{array}$ & $\begin{array}{l}c^{3} \mathrm{~F}_{4}-t{ }^{3} \mathrm{D}_{3}^{0} \\
c^{3} \mathrm{~F}_{3}-t{ }^{3} \mathrm{D}_{3}^{0} \\
d^{3} \mathrm{~F}_{2}-u^{3} \mathrm{~F}_{2}^{\circ} \\
d^{3} \mathrm{~F}_{3}-u^{3} \mathrm{~F}_{2}^{\circ}\end{array}$ & & \\
\hline $\begin{array}{l}2190.48 \\
2190.22 \\
2188.86 \\
2186.94 \\
2185.96\end{array}$ & $\begin{array}{l}2 \\
3\end{array}$ & $\begin{array}{r}8 \\
30 \\
2 \\
20 \\
40\end{array}$ & & $\begin{array}{l}45637.78 \\
45643.20 \\
45671.56 \\
45711.65 \\
45732.14\end{array}$ & $\begin{array}{l}b^{3} \mathrm{P}_{1}-v^{3} \mathrm{D}_{3}^{\circ} \\
b^{3} \mathrm{G}_{5}-w^{3} \mathrm{~F}_{4}^{\circ} \\
b^{3} \mathrm{P}_{2}-v^{3} \mathrm{D}_{2}^{8} \\
b^{3} \mathrm{G}_{4}-w^{3} \mathrm{~F}_{3}^{\circ} \\
d^{3} \mathrm{~F}_{2}-22_{3}^{\circ}\end{array}$ & & \\
\hline
\end{tabular}

See footnotes at end of table. 
TABLE 1.-Second spectrum of Vanadium-Continued

\begin{tabular}{|c|c|c|c|c|c|c|c|}
\hline \multirow{3}{*}{$\lambda_{\mathrm{air}} \mathrm{A}$} & \multicolumn{2}{|c|}{2} & 3 & 4 & 5 & & \\
\hline & & nsity & \multirow{2}{*}{$\begin{array}{c}\text { Temp. } \\
\text { class }\end{array}$} & \multirow{2}{*}{$\begin{array}{l}\text { Wave } \\
\text { No. vac }\end{array}$} & \multirow{2}{*}{$\begin{array}{c}\text { Term } \\
\text { combination }\end{array}$} & \multicolumn{2}{|c|}{ Zeeman effect } \\
\hline & Arc & Spark & & & & Observed & Computed \\
\hline 2185.39 & 4 & 50 & & 45744.06 & $\left\{\begin{array}{l}b^{3} \mathrm{G}_{3}-w^{3} \mathrm{~F}_{2}^{\circ} \\
d^{3} \mathrm{~F}_{3}-u^{3} \mathrm{~F}_{3}^{0}\end{array}\right.$ & & \\
\hline $\begin{array}{l}2184.89 \\
2184.41 \\
2184.17 \\
2183.08\end{array}$ & & $\begin{array}{l}2 \\
2 \\
5 \\
2\end{array}$ & & $\begin{array}{l}45754.53 \\
45764.58 \\
45769.61 \\
45792.46\end{array}$ & $\begin{array}{l}b^{3} \mathrm{G}_{4}-w{ }^{3} \mathrm{~F}^{4} \\
b^{3} \mathrm{P}_{1}-v{ }^{3} \mathrm{Di}_{1} \\
b^{3} \mathrm{P}_{0}-v{ }^{3} \mathrm{D}_{1}^{1} \\
b^{3} \mathrm{G}_{3}-w^{3} \mathrm{~F}_{3}^{3}\end{array}$ & & \\
\hline $\begin{array}{l}2175.833 \\
2171.840\end{array}$ & & $\begin{array}{r}5 \\
25\end{array}$ & & $\begin{array}{l}45944.96 \\
46029.42\end{array}$ & $d^{3} \mathrm{~F}_{4}-u^{3} \mathrm{~F}_{4}^{0}$ & & \\
\hline 2171.12 & & 2 & & 46044.4 & $\left\{\begin{array}{l}a^{3}{ }^{3} \mathrm{~F}_{4}-y^{1}{ }^{1} \mathrm{~F}_{3}^{o} \\
b^{3} \mathrm{D}_{3}-y^{3} \mathrm{G}_{3}^{\circ}\end{array}\right.$ & & \\
\hline $\begin{array}{l}2170.38 \\
2170.05\end{array}$ & & $\begin{array}{l}15 \\
10 H\end{array}$ & & $\begin{array}{l}46060.4 \\
46067.4\end{array}$ & ${ }^{3} \mathrm{~F}_{4}-u^{3} \mathrm{~F}_{3}^{\circ}$ & & \\
\hline $\begin{array}{l}2168.56 \\
2168.08 \\
2167.69 \\
2166.15 \\
2164.38\end{array}$ & & $\begin{array}{c}0 h \\
10 \\
8 \\
20 h \\
15\end{array}$ & & $\begin{array}{l}46099.0 \\
46109.2 \\
46117.5 \\
46150.3 \\
46188.1\end{array}$ & $\begin{array}{l}c^{3} \mathrm{~F}_{3}-2_{3}^{\circ} \\
a^{3} \mathrm{~F}_{4}-x^{3} \mathrm{G}_{5}^{\circ} \\
c^{3} \mathrm{~F}_{2}-u^{3} \mathrm{~F}_{3}^{\circ}\end{array}$ & & \\
\hline $\begin{array}{l}2163.68 \\
2161.48 \\
2160.55 \\
2159.53\end{array}$ & & $\begin{array}{c}20 h \\
20 \\
5 \\
0 h\end{array}$ & & $\begin{array}{l}46203.0 \\
46250.0 \\
46269.9 \\
46291.8\end{array}$ & $\begin{array}{l}a^{3} \mathrm{D}_{3}-v^{3} \mathrm{D}_{3}^{\circ} \\
a^{3} \mathrm{D}_{2}-x{ }^{1} \mathrm{D}_{2}^{\circ}\end{array}$ & & \\
\hline 2159.13 & & OhFeII? & & 46300.4 & $\left\{\begin{array}{l}a^{5} \mathrm{~F}_{4}-y^{3} \mathrm{~F}_{4}^{0} \\
a^{3} \mathrm{~F}_{3}-y^{1} \mathrm{~F}_{3}^{0}\end{array}\right.$ & & \\
\hline 2158. 36 & & $\begin{array}{l}0 H \\
8\end{array}$ & & $\begin{array}{l}46316.9 \\
46345.0\end{array}$ & $a^{3} \mathrm{D}_{1}-x{ }^{1} \mathrm{D}_{2}^{\circ} ?$ & & \\
\hline $\begin{array}{l}2155.61 \\
2154.42 \\
2151.812\end{array}$ & 5 & $\begin{array}{r}0 \\
15 \\
0 \\
50\end{array}$ & & $\begin{array}{l}46345.0 \\
46375.9 \\
46401.6 \\
46457.79\end{array}$ & $\begin{array}{l}a{ }^{1} \mathrm{G}_{4}-y{ }^{5} \mathrm{~F}_{5}^{\circ} \\
a^{3} \mathrm{~F}_{4}-y^{1} \mathrm{H}_{5}^{\circ}\end{array}$ & & \\
\hline $\begin{array}{l}2151.032 \\
2150.835 \\
2149.386 \\
2148.42 \\
2148.00\end{array}$ & $\begin{array}{r}5 \\
6 \\
15\end{array}$ & $\begin{array}{r}50 \\
60 \\
8 \\
40 \\
8\end{array}$ & & $\begin{array}{l}46474.64 \\
46478.89 \\
46510.22 \\
46531.1 \\
46540.3\end{array}$ & $\begin{array}{l}a^{3} \mathrm{D}_{2}-v{ }^{3} \mathrm{D}_{2}^{\circ} \\
a^{5} \mathrm{D}_{3}-z^{3} \mathrm{P}_{2}^{\circ}\end{array}$ & & \\
\hline $\begin{array}{l}2147.52 \\
2145.990 \\
2145.37^{*} \\
2144.10 \\
2143.706\end{array}$ & 10 & $\begin{array}{l}20 \\
40 \\
0 h \\
5 \\
5\end{array}$ & & $\begin{array}{l}46550.6 \\
46583.8 \\
46597.3 \\
46624.9 \\
46633.44\end{array}$ & $\begin{array}{l}a^{5}{ }^{5} \mathrm{D}_{1}-z{ }^{3} \mathrm{P}_{0}^{0} \\
a^{5} \mathrm{D}_{2}-z \\
a^{3} \mathrm{P}_{1}^{6} \mathrm{~F}_{1}-y^{3} \mathrm{~F}_{2}^{2} ? \\
a^{5} \mathrm{~b}_{4}-z{ }^{1} \mathrm{H}_{5}^{5} \\
a^{5} \mathrm{D}_{2}-z^{3} \mathrm{P}_{2}^{5}\end{array}$ & & \\
\hline $\begin{array}{l}2143.038 \\
2142.74 \\
2142.40 \\
2141.973 \\
2141.70\end{array}$ & $\begin{array}{r}30 \\
1 \\
40 \\
0\end{array}$ & $\begin{array}{c}60 \\
4 \\
3 \\
100 \\
4 h ?\end{array}$ & & $\begin{array}{l}46647.98 \\
46654.5 \\
46661.9 \\
46671.17 \\
46677.1\end{array}$ & $\begin{array}{l}a^{5} \mathrm{D}_{2}-z{ }^{5} \mathrm{P}_{1}^{\circ} \\
a^{5} \mathrm{D}_{1}-z \\
a^{3}{ }^{3} \mathrm{P}_{1}^{1} \\
a^{5} \mathrm{D}_{1}-v{ }^{3} \mathrm{D}_{1}^{1} \\
b^{5} \mathrm{D}_{3}-z{ }^{5} \mathrm{P}_{2}^{0} \\
b^{1} \mathrm{G}_{4}-x x^{1} \mathrm{G}_{4}^{3}\end{array}$ & & \\
\hline $\begin{array}{l}2140.064 \\
2139.798 \\
2138.17 \\
2137.31 \\
2134.12\end{array}$ & $\begin{array}{r}60 \\
40 \\
30 \\
50 \\
100\end{array}$ & $\begin{array}{r}150 \\
100 \\
60 \\
100 \\
200\end{array}$ & & $\begin{array}{l}46712.79 \\
46718.60 \\
46754.2 \\
46773.0 \\
46842.9\end{array}$ & $\begin{array}{l}a^{5} \mathrm{D}_{4}-z^{5} \mathrm{P}_{3}^{0} \\
a^{5} \mathrm{D}_{1}-z{ }^{5} \mathrm{P}_{1}^{0} \\
a^{5} \mathrm{D}_{0}-z{ }^{5} \mathrm{P}_{1}^{0} \\
a^{5} \mathrm{D}_{2}-z{ }^{5} \mathrm{P}_{2}^{0} \\
a^{5} \mathrm{D}_{3}-z{ }^{5} \mathrm{P}_{3}^{0}\end{array}$ & & \\
\hline $\begin{array}{l}2133.04 \\
2131.85 \\
2130.85 \\
2130.42 \\
2129.477\end{array}$ & 10 & $\begin{array}{r}60 \\
80 \\
0 \\
5 \\
40\end{array}$ & & $\begin{array}{l}46866.6 \\
46892.8 \\
46914.8 \\
46924.2 \\
46945.01\end{array}$ & $\begin{array}{l}a^{5} \mathrm{D}_{3}-y^{5} \mathrm{D}_{2}^{\circ} \\
a^{5} \mathrm{~F}_{3}-z^{1} \mathrm{G}_{4}^{\circ} \\
a^{1} \mathrm{D}_{2}-v^{3} \mathrm{~F}_{3}^{\circ} \\
a^{5} \mathrm{D}_{2}-z^{5} \mathrm{P}_{3}^{\circ}\end{array}$ & & \\
\hline $\begin{array}{l}2128.241 \\
2127.34 \\
2126.932 \\
2126.585 \\
2124.00\end{array}$ & $\begin{array}{l}1 \\
1 \\
6\end{array}$ & $\begin{array}{c}7 \\
5 \\
20 \\
25 h \\
5 d ?\end{array}$ & & $\begin{array}{l}46972.26 \\
46992.2 \\
47001.17 \\
47008.84 \\
47066.0\end{array}$ & $\begin{array}{l}a^{5} \mathrm{D}_{3}-y{ }^{5} \mathrm{D}_{3}^{\circ} \\
a^{5} \mathrm{D}_{1}-y \\
a^{5} \mathrm{D}_{2} \mathrm{D}_{0}^{\circ}-y^{5} \mathrm{D}_{1}^{\circ} \\
a^{5} \mathrm{D}_{1}-y^{5} \mathrm{D}_{2}^{\circ}\end{array}$ & & \\
\hline $\begin{array}{l}2123.62 \\
2123.340 \\
2122.11 \\
2121.54 \\
2119.562\end{array}$ & $\begin{array}{c}3 \\
20 \\
1 ?\end{array}$ & $\begin{array}{r}10 \\
60 \\
1 \\
10 \\
15\end{array}$ & & $\begin{array}{l}47074.5 \\
47080.67 \\
47108.0 \\
47120.6 \\
47164.58\end{array}$ & 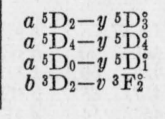 & & \\
\hline
\end{tabular}

See footnotes at end of table. 
TABLE 1.-Second spectrum of Vanadium-Continued

\begin{tabular}{|c|c|c|c|c|c|c|c|}
\hline \multirow{3}{*}{$\lambda_{\mathrm{s} \text { ir }} \mathbf{A}$} & \multicolumn{2}{|c|}{2} & 3 & 4 & 5 & & \\
\hline & & ensity & \multirow{2}{*}{$\begin{array}{l}\text { Temp. } \\
\text { class }\end{array}$} & \multirow{2}{*}{$\begin{array}{l}\text { Wave } \\
\text { No. vac }\end{array}$} & \multirow{2}{*}{$\begin{array}{c}\text { Term } \\
\text { combination }\end{array}$} & \multicolumn{2}{|c|}{ Zeeman effect } \\
\hline & Arc & Spark & & & & Observed & Computed \\
\hline $\begin{array}{l}2119.15 \\
2118.84 \\
2118.43^{*} \\
2117.482 \\
2117.293\end{array}$ & $\begin{array}{l}1 \\
4\end{array}$ & $\begin{array}{l}40 h \\
25 \\
30 h \\
12 \\
25\end{array}$ & & $\begin{array}{l}47173.7 \\
47180.6 \\
47189.8 \\
47210.90 \\
47215.12\end{array}$ & $\begin{array}{l}b^{1} \mathrm{G}_{4}-x^{1} \mathrm{~F}_{3}^{0} \\
a^{5} \mathrm{D}_{3}-y^{5} \mathrm{D}_{4}^{\circ} \\
b^{3} \mathrm{D}_{1}-v^{3} \mathrm{~F}_{2}^{\circ}\end{array}$ & & \\
\hline $\begin{array}{l}2114.30 \\
2114.03 \\
2111.04 \\
2110.48 \\
2109.27\end{array}$ & & $\begin{array}{r}15 \\
30 \\
15 \\
5 \\
8\end{array}$ & & $\begin{array}{l}47281.9 \\
47288.0 \\
47354.9 \\
47367.5 \\
47394.7\end{array}$ & $\begin{array}{l}b^{3} \mathrm{D}_{3}-v{ }^{3} \mathrm{~F}_{3}^{\circ} \\
b^{3} \mathrm{D}_{2}-v{ }^{3} \mathrm{~F}_{3}^{8} \\
c^{8} \mathrm{P}_{2}-v^{3} \mathrm{~F}_{2}^{\circ}\end{array}$ & & \\
\hline $\begin{array}{l}2107.40 \\
2103.70 \\
2103.53 \\
2101.86 \\
2101.17\end{array}$ & $\begin{array}{r}8 \\
10 \\
t r \\
5\end{array}$ & $\begin{array}{l}10 h \\
80 \\
30 \\
20 \\
50\end{array}$ & & $\begin{array}{l}47436.7 \\
47520.2 \\
47524.0 \\
47561.8 \\
47577.4\end{array}$ & $\begin{array}{l}a^{3} \mathrm{D}_{3}-x^{1} \mathrm{G}_{4} \\
a^{3} \mathrm{~F}_{3}-w^{3} \mathrm{~F}_{4} \\
b^{3} \mathrm{D}_{3}-v^{3} \mathrm{~F}_{4}^{\circ} \\
c^{3} \mathrm{P}_{2}-v^{3} \mathrm{~F}_{3}^{3} \\
a^{3} \mathrm{G}_{4}-w^{3} \mathrm{~F}_{3}^{3}\end{array}$ & & \\
\hline $\begin{array}{l}2100.25 \\
2099.47 \\
2099.16 \\
2098.60 \\
2098.00\end{array}$ & 3 & $\begin{array}{c}0 \\
0 \\
30 \\
0 \\
5 d ?\end{array}$ & & $\begin{array}{l}47598.2 \\
47615.9 \\
47622.9 \\
47635.6 \\
47649.2\end{array}$ & $\begin{array}{c}b^{3} \mathrm{G}_{5}-w^{3} \mathrm{G}_{4} \\
a^{3} \mathrm{D}_{1}-x^{5} \mathrm{D}_{2}^{2} \\
a^{3} \mathrm{G}_{3}-w^{3} \mathrm{~F}_{2}^{2} \\
b^{3} \mathrm{G}_{4}-w^{3} \mathrm{G}_{3}^{3} \\
c^{3} \mathrm{P}_{1}-v^{3} \mathrm{~F}_{2}^{2} ?\end{array}$ & & \\
\hline $\begin{array}{l}2097.03 \\
2095.94 \\
2095.37 \\
2095.05 \\
2091.81\end{array}$ & $\begin{array}{l}0 \\
5\end{array}$ & $\begin{array}{c}2 \\
25 \\
15 \\
15 \\
2 h\end{array}$ & & $\begin{array}{l}47671.3 \\
47696.1 \\
47709.0 \\
47716.3 \\
47790.2\end{array}$ & $\begin{array}{l}a^{3} \mathrm{G}_{3}-w^{3} \mathrm{~F}_{3}^{3} \\
b^{3} \mathrm{G}_{5}-w^{3} \mathrm{G}_{5}^{8} \\
b^{3} \mathrm{G}_{4}-w^{3} \mathrm{G}_{4}^{8} \\
b^{3} \mathrm{G}_{3}-w^{3} \mathrm{G}_{3}^{3} \\
b^{3} \mathrm{G}_{3}-w^{3} \mathrm{G}_{4}^{8}\end{array}$ & . & \\
\hline $\begin{array}{l}2090.33 \\
2087.92 \\
2087.54 \\
2079.29 \\
2077.79\end{array}$ & $t r ?$ & $\begin{array}{l}25 \\
20 \\
15 \\
10 H \\
40 H\end{array}$ & & $\begin{array}{l}47824.1 \\
47879.3 \\
47888.0 \\
48077.9 \\
48112.7\end{array}$ & $\begin{array}{l}a{ }^{1} \mathrm{~F}_{3}-w \\
a^{1} \mathrm{G}_{4}-x^{1} \mathrm{~F}_{3}\end{array}$ & & \\
\hline $\begin{array}{l}2077.58 \\
2076.87 \\
2076.52 \\
2075.13 \\
2074.87\end{array}$ & $\begin{array}{c}t r ? \\
30 \\
0 ?\end{array}$ & $\begin{array}{l}15 H \\
60 h \\
0 ? ? \\
15 \\
25\end{array}$ & & $\begin{array}{l}48117.5 \\
48134.0 \\
48142.1 \\
48174.3 \\
48180.4\end{array}$ & $\begin{array}{l}d^{3} \mathrm{~F}_{2}-w^{1} \mathrm{D}_{2} \\
b^{3} \mathrm{D}_{2}-u^{3} \mathrm{D}_{1}^{3} \\
b^{3} \mathrm{D}_{3}-u^{3} \mathrm{D}_{2}^{3} \\
b^{3} \mathrm{D}_{2}-u^{3} \mathrm{D}_{2}^{8}\end{array}$ & & \\
\hline $\begin{array}{l}2072.43 \\
2070.79 \\
2068.80 \\
2068.54 \\
2066.83\end{array}$ & $\begin{array}{c}t r \\
10 \\
0\end{array}$ & $\begin{array}{c}30 \\
15 \\
60 \\
15 \\
8 h\end{array}$ & & $\begin{array}{l}48237.1 \\
48275.3 \\
48321.7 \\
48327.8 \\
48367.8\end{array}$ & $\begin{array}{l}b^{3} \mathrm{D}_{1}-u{ }^{3} \mathrm{D}_{1}^{\circ} \\
b^{3} \mathrm{D}_{1}-u{ }^{3} \mathrm{D}_{2}^{\circ} \\
b^{3} \mathrm{D}_{2}-u{ }^{3} \mathrm{D}_{3}^{\circ} \\
b^{3} \mathrm{D}_{2}-u^{3} \mathrm{D}_{3}^{\circ} \\
d^{3} \mathrm{~F}_{2}-3_{3}^{3}\end{array}$ & & \\
\hline $\begin{array}{l}2065.76 \\
2064.78 \\
2063.12 \\
2062.00 \\
2061.56\end{array}$ & $t r$ & $\begin{array}{r}40 \\
2 \\
20 \\
10 \\
15\end{array}$ & & $\begin{array}{l}48392.8 \\
48415.8 \\
48454.7 \\
48481.0 \\
48491.4\end{array}$ & $\begin{array}{l}a^{1} \mathrm{G}_{4}-x^{1} \mathrm{~F}_{3}^{\circ} \\
c^{3} \mathrm{P}_{2}-u^{3} \mathrm{D}_{1}^{\circ} \\
c^{3} \mathrm{P}_{2}-u^{3} \mathrm{D}_{2}^{\circ}\end{array}$ & & \\
\hline $\begin{array}{l}2058.34 \\
2057.36 \\
2057.20\end{array}$ & $\begin{array}{r}10 \\
8 \\
5\end{array}$ & $\begin{array}{l}40 \\
25 \\
15\end{array}$ & & $\begin{array}{l}48567.2 \\
48590.4 \\
48594.1\end{array}$ & $\begin{array}{l}b^{3} \mathrm{~F}_{4}-w^{3} \mathrm{~F}_{4}^{0} \\
b^{3} \mathrm{~F}_{3}-w^{3} \mathrm{~F}_{3}^{3} \\
b^{3} \mathrm{~F}_{2}-w^{3} \mathrm{~F}_{2}^{2}\end{array}$ & & \\
\hline 2056.89 & & 15 & & 48601.5 & $\left\{\begin{array}{l}c^{3}{ }^{3} \mathrm{P}_{2}-u^{3} \mathrm{D}_{3}^{2} \\
c^{3} \mathrm{P}_{0}-u^{3} \mathrm{D}_{1}^{3}\end{array}\right.$ & & \\
\hline 2055.55 & & 8 & & 48633.1 & $b^{3} \mathrm{~F}_{3}-20^{3} \mathrm{~F}_{4}^{0}$ & & \\
\hline $\begin{array}{l}2055.15 \\
2054.85 \\
2054.27 \\
2054.01 \\
2052.38\end{array}$ & 10 & $\begin{array}{c}5 \\
70 \\
0 h \\
0 \\
10\end{array}$ & & $\begin{array}{l}48642.6 \\
48649.7 \\
48663.4 \\
48669.6 \\
48708.2\end{array}$ & $\begin{array}{c}b^{3} \mathrm{~F}_{2}-w^{3} \mathrm{~F}_{3}^{\circ} \\
a{ }^{1} \mathrm{D}_{2}-v^{3} \mathrm{G}_{3}^{3} \\
c^{3} \mathrm{P}_{1}-u^{3} \mathrm{D}_{1}^{1} \\
c^{3} \mathrm{P}_{1}-u^{3} \mathrm{D}_{2}^{2}\end{array}$ & & \\
\hline $\begin{array}{l}\text { 2051. } 79 \\
\text { 2051. } 27 \\
2049.67\end{array}$ & & $\begin{array}{r}30 h \\
5 h \\
5 h\end{array}$ & & $\begin{array}{l}48722.2 \\
48734.6 \\
48772.6\end{array}$ & $\begin{array}{l}c^{3} \mathrm{~F}_{4}-3_{3}^{\circ} \\
c^{3} \mathrm{~F}_{3}-3_{3}^{\circ} \\
b^{3} \mathrm{P}_{2}-v^{3} \mathrm{~F}_{3}^{\circ} ? \\
c^{3} \mathrm{~F}_{2}-3_{3}^{\circ}\end{array}$ & & \\
\hline $\begin{array}{l}2048.75 \\
2046.12\end{array}$ & $t r$ & $\begin{array}{r}15 \\
0\end{array}$ & & $\begin{array}{l}48794.5 \\
48857.2\end{array}$ & $a^{1} \mathrm{~S}_{0}-u{ }^{3} \mathrm{D}_{\mathrm{i}}$ & & \\
\hline
\end{tabular}

See footnotes at end of table. 
TABLE 1.-Second spectrum of Vanadium-Continued

\begin{tabular}{|c|c|c|c|c|c|c|c|}
\hline 1 & & 2 & 3 & 4 & 5 & \multicolumn{2}{|c|}{6} \\
\hline \multirow{2}{*}{$\lambda_{\mathrm{B} \text { ir }} \mathrm{A}$} & \multicolumn{2}{|c|}{ Intensity } & \multirow{2}{*}{$\begin{array}{l}\text { Temp. } \\
\text { class }\end{array}$} & \multirow{2}{*}{$\begin{array}{l}\text { Wave } \\
\text { No. vac }\end{array}$} & \multirow{2}{*}{$\begin{array}{c}\text { Term } \\
\text { combination }\end{array}$} & \multicolumn{2}{|c|}{ Zeeman effect } \\
\hline & Arc & Spark & & & & Observed & Computed \\
\hline $\begin{array}{l}2044.28 \\
2039.29 \\
2037.83 \\
2037.50 \\
2035.78\end{array}$ & $2 h$ & $\begin{array}{r}5 \\
60 \\
50 \\
25 \\
15\end{array}$ & & $\begin{array}{l}48901.2 \\
49020.9 \\
49056.0 \\
49063.9 \\
49105.4\end{array}$ & $a_{a}^{a}{ }^{1} \mathrm{H}_{s}-w^{1}{ }^{1} \mathrm{G}_{4}^{\circ}$ & & \\
\hline $\begin{array}{l}2035.06 \\
2033.50 \\
2031.40 \\
2028.88 \\
2025.47\end{array}$ & & $\begin{array}{l}60 \\
10 \\
30 h \\
15 \\
15\end{array}$ & & $\begin{array}{l}49122.7 \\
49160.4 \\
49211.2 \\
49272.3 \\
49355.3\end{array}$ & $a^{3} \mathrm{G}_{3}-y^{5} \mathrm{~F}_{3}^{\circ}$ & & \\
\hline $\begin{array}{l}2024.84 \\
2023.56 \\
2022.66 \\
2021.83 \\
2021.38\end{array}$ & 10 & $\begin{array}{c}0 ? ? \\
50 \\
15 h \\
5 \\
10 h\end{array}$ & & $\begin{array}{l}49370.6 \\
49401.9 \\
49423.9 \\
49444.1 \\
49455.1\end{array}$ & $\begin{array}{l}a^{3} \mathrm{G}_{5}-v^{5} \mathrm{~F}_{4}^{\circ} ? \\
b^{3} \mathrm{H}_{4}-v^{3} \mathrm{G}_{3}^{\circ} \\
a^{3} \mathrm{D}_{2}-v^{3} \mathrm{~F}_{2}^{\circ}\end{array}$ & & \\
\hline $\begin{array}{l}2020.83 \\
2020.54 \\
2019.47 \\
2017.46 \\
2017.32\end{array}$ & $\begin{array}{l}0 \\
0 h \\
t r\end{array}$ & $\begin{array}{r}15 \\
10 \\
10 \\
2 \\
2\end{array}$ & & $\begin{array}{l}49468.6 \\
49475.7 \\
49501.9 \\
49551.2 \\
49554.6\end{array}$ & $\begin{array}{l}a^{3} \mathrm{D}_{1}-v^{3} \mathrm{~F}_{2}^{\circ} \\
a^{3} \mathrm{G}_{5}-w^{3} \mathrm{G}_{4} \\
a^{3} \mathrm{G}_{4}-w^{3} \mathrm{G}_{3}^{3} \\
a^{3} \mathrm{D}_{3}-v^{3} \mathrm{~F}^{3} \\
a^{3} \mathrm{H}_{5}-w^{3} \mathrm{~F}_{4}^{8}\end{array}$ & & \\
\hline 2016. 53 & 15 & 60 & & 49574.1 & $\left\{\begin{array}{l}a^{3} \mathrm{G}_{5}-w^{3} \mathrm{G}_{5}^{\circ} \\
a^{3} \mathrm{G}_{4}-w^{3} \mathrm{G}_{4}^{\circ}\end{array}\right.$ & & \\
\hline 2015.74 & tr & 20 & & 49593.5 & $\left\{\begin{array}{c}a^{3} \mathrm{G}_{3}-w^{3}{ }^{3} \mathrm{G}_{3}^{0} \\
b^{3} \mathrm{P}_{1}-u^{3} \mathrm{D}_{1}^{0}\end{array}\right.$ & & \\
\hline $\begin{array}{l}2015.56 \\
2015.02\end{array}$ & $\stackrel{t r}{0 h}$ & $\begin{array}{l}20 \\
15\end{array}$ & & $\begin{array}{l}49597.9 \\
49611.2\end{array}$ & $\begin{array}{c}b^{3} \mathrm{P}_{0}-u u^{3} \mathrm{D}^{1} \\
a^{3} \mathrm{D}_{2}-v^{3} \mathrm{~F}_{3}^{0} \\
b^{3} \mathrm{P}_{1}-u^{3} \mathrm{D}_{2}^{0}\end{array}$ & & \\
\hline 2014.18 & 20 & 90 & & 49631.9 & $\left\{\begin{array}{l}a^{3} \mathrm{G}_{5}-y \mathrm{~F}_{5}^{5} \\
b^{3} \mathrm{H}_{5}-v^{3} \mathrm{G}_{4}^{0}\end{array}\right.$ & & \\
\hline 2012.84 & $t r$ & 20 & & 49664.9 & $b^{3} \mathrm{P}_{2}-u{ }^{3} \mathrm{D}_{2}^{\circ}$ & & \\
\hline 2012. 64 & $0 h$ & 10 & & 49669.9 & $\left\{\begin{array}{l}b^{b}{ }^{3} \mathrm{H}_{4}-v^{3} \mathrm{G}_{4}^{4} \\
a^{3} \mathrm{G}_{3}-w^{3} \mathrm{G}_{4}\end{array}\right.$ & & \\
\hline $\begin{array}{l}2010.15 \\
2007.66 \\
2006.88\end{array}$ & $\begin{array}{c}3 \\
t r \\
10\end{array}$ & $\begin{array}{l}5 h \\
25 \\
80\end{array}$ & & $\begin{array}{l}49731.4 \\
49793.0 \\
49812.4\end{array}$ & $\begin{array}{c}a^{3} \mathrm{G}_{4}-y^{5} \mathrm{~F}_{5}^{0} \\
a^{3} \mathrm{D}_{3}-v^{3} \mathrm{~F}_{4}^{0} \\
b^{3} \mathrm{P}_{2}-u^{3} \mathrm{D}_{3}^{3}\end{array}$ & & \\
\hline $\begin{array}{l}2006.08 \\
2005.88 \\
2004.77 \\
2004.03 \\
2002.82\end{array}$ & 15 & $\begin{array}{c}0 ? ? \\
15 \\
90 \\
0 \\
0\end{array}$ & & $\begin{array}{l}49832.3 \\
49837.2 \\
49864.8 \\
49883.2 \\
49913.4\end{array}$ & $\begin{array}{l}b^{1} \mathrm{G}_{4}-u^{3} \mathrm{D}_{3}^{\circ} \\
b^{3} \mathrm{H}_{6}-v^{3} \mathrm{G}_{5}^{\circ} \\
b^{3} \mathrm{G}_{4}-x^{1} \mathrm{~F}_{3}^{\circ} ? \\
a^{5} \mathrm{~F}_{5}-z^{3} \mathrm{I}_{6}^{\circ}\end{array}$ & & \\
\hline $\begin{array}{l}2001.65 \\
2001.43 \\
2001.14 \\
2000.78 \\
2000.14\end{array}$ & $\begin{array}{l}t r \\
t r\end{array}$ & $\begin{array}{c}40 \\
10 \\
30 \\
5 \\
10 h\end{array}$ & & $\begin{array}{l}49942.5 \\
49948.0 \\
49955.3 \\
49964.2 \\
49980.2\end{array}$ & $\begin{array}{l}b^{3} \mathrm{H}_{5}-v^{3} \mathrm{G}_{5}^{\circ} \\
b^{3} \mathrm{G}_{3}-x^{1} \mathrm{~F}_{3}^{\circ} ?\end{array}$ & & \\
\hline $\begin{array}{r}\lambda V a c \\
1995.02 \\
1992.80 \\
1987.82 \\
1984.05 \\
1982.41\end{array}$ & 10 & $\begin{array}{r}0 \\
30 \\
3 \\
90 \\
8\end{array}$ & & $\begin{array}{l}50124.8 \\
50180.7 \\
50306.4 \\
50402.0 \\
50443.7\end{array}$ & $\begin{array}{l}a^{3} \mathrm{G}_{3}-x^{1} \mathrm{D}_{2}^{2} \\
a^{3} \mathrm{D}_{3}-u^{3} \mathrm{D}_{2}^{2}\end{array}$ & & \\
\hline $\begin{array}{l}1982-21 \\
1981.53 \\
1980.59 \\
1980.04 \\
1978.96\end{array}$ & $\begin{array}{l}0 \\
0 \\
0\end{array}$ & $\begin{array}{r}0 \\
8 \\
25 \\
40 \\
20\end{array}$ & & $\begin{array}{l}50448.7 \\
50466.1 \\
50490.0 \\
50504.0 \\
50531.6\end{array}$ & $\begin{array}{l}b^{3} \mathrm{~F}_{4}-w^{3} \mathrm{G}_{3}^{\circ} \\
a^{3} \mathrm{D}_{2}-u^{3} \mathrm{D}_{1}^{\circ} \\
a^{3} \mathrm{D}_{1}-u^{3} \mathrm{D}_{1}^{\circ} \\
a^{3} \mathrm{D}_{2}-u^{3} \mathrm{D}_{2}^{\circ} \\
b^{1} \mathrm{G}_{4}-v^{3} \mathrm{G}_{3}^{\circ}\end{array}$ & & \\
\hline $\begin{array}{l}1977.60 \\
1976.62 \\
1972.62 \\
1960.98 \\
1950.77\end{array}$ & $\begin{array}{l}1 \\
0\end{array}$ & $\begin{array}{l}0 ? ? \mathrm{I} ? \\
60 \\
20 \\
2 \\
0\end{array}$ & & $\begin{array}{l}50566.3 \\
50591.4 \\
50694.0 \\
50994.9 \\
51261.8\end{array}$ & 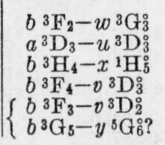 & & \\
\hline
\end{tabular}

See footnotes at end of table. 
TABLE 1.-Second specirum of Vanadium-Continued

\begin{tabular}{|c|c|c|c|c|c|c|c|}
\hline 1 & & 2 & 3 & 4 & 5 & & \\
\hline \multirow{2}{*}{$\lambda_{\mathrm{air}} \mathrm{A}$} & \multicolumn{2}{|c|}{ Intensity } & \multirow{2}{*}{$\begin{array}{l}\text { Temp. } \\
\text { class }\end{array}$} & \multirow{2}{*}{$\begin{array}{l}\text { Wave } \\
\text { No. vac }\end{array}$} & \multirow{2}{*}{$\begin{array}{c}\text { Term } \\
\text { combination }\end{array}$} & \multicolumn{2}{|c|}{ Zeeman effect } \\
\hline & Arc & Spark & & & & Observed & Computed \\
\hline $\begin{array}{l}1945.64 \\
1945.35\end{array}$ & 3 & $\begin{array}{l}30 \\
30 B l \mathrm{Fe} \text { III? }\end{array}$ & & $\begin{array}{l}51397.0 \\
51404.6\end{array}$ & $\begin{array}{l}b^{3} \mathrm{G}_{3}-v^{3} \mathrm{~F}_{2} \\
a^{3} \mathrm{H}_{5}-y^{5} \mathrm{~F}_{4}^{0}\end{array}$ & & \\
\hline 1943.99 & & tr & & 51440.6 & $\left\{\begin{array}{l}a^{3} \mathrm{G}_{4}-x^{5} \mathrm{D}_{3}^{2} \\
b^{3} \mathrm{~F}_{2}-v^{3} \mathrm{D}_{1}^{1}\end{array}\right.$ & & \\
\hline $\begin{array}{l}1942.35 \\
1941.40\end{array}$ & $\begin{array}{r}8 \\
10\end{array}$ & $\begin{array}{l}40 \\
30\end{array}$ & & $\begin{array}{l}51484.0 \\
51509.2\end{array}$ & $\begin{array}{l}b^{3} \mathrm{G}_{4}-v^{3} \mathrm{~F}_{3}^{2} \\
a^{3} \mathrm{H}_{5}-w^{3} \mathrm{G}_{4}\end{array}$ & & \\
\hline $\begin{array}{l}1941.27 \\
1940.86 \\
1939.32 \\
1938.70 \\
1938.50\end{array}$ & $\begin{array}{l}10 \\
10\end{array}$ & $\begin{array}{r}30 \\
40 \\
8 \\
8 \\
10\end{array}$ & & $\begin{array}{l}51512.7 \\
51523.6 \\
51564.5 \\
51581.0 \\
51586.3\end{array}$ & $\begin{array}{c}a^{3} \mathrm{H}_{4}-w^{3} \mathrm{G}_{3}^{\circ} \\
a^{3} \mathrm{H}_{6}-w^{3} \mathrm{G}_{5}^{5} \\
b^{3} \mathrm{G}_{3}-v^{3} \mathrm{~F}_{3}^{5} \\
a^{3} \mathrm{H}_{8}-y^{5} \mathrm{~F}_{5}^{5} \\
a^{3} \mathrm{H}_{4}-w^{3} \mathrm{G}_{4}^{\circ}\end{array}$ & & \\
\hline $\begin{array}{l}\text { 1937. } 68 \\
1937.44 \\
1933.97 \\
1933.28 \\
1932.99\end{array}$ & 10 & $\begin{array}{r}7 \\
60 \\
30 \\
10 \\
3\end{array}$ & & $\begin{array}{l}51608.1 \\
51614.5 \\
51707.1 \\
51725.6 \\
51733.3\end{array}$ & $\begin{array}{c}a^{3} \mathrm{H}_{5}-w^{3} \mathrm{G}_{5}^{\circ} \\
b^{3} \mathrm{G}_{5}-v^{3} \mathrm{~F}_{4}^{\circ} \\
b^{3} \mathrm{G}_{4}-v^{3} \mathrm{~F}_{4}^{\circ} \\
a^{1} \mathrm{G}_{4}-v^{3} \mathrm{G}_{3}^{\circ}\end{array}$ & & \\
\hline $\begin{array}{l}1932.55 \\
1929.61 \\
1924.87 \\
1921.24 \\
1920.36\end{array}$ & 10 & \begin{tabular}{l}
\multicolumn{1}{c}{${ }^{\circ}$} \\
30 \\
$15 h e ?$ \\
12
\end{tabular} & & $\begin{array}{l}51745.1 \\
51823.9 \\
51951.6 \\
52049.7 \\
52073.6\end{array}$ & $\begin{array}{c}a^{1} \mathrm{I}_{6}-x^{1} \mathrm{H}_{5}^{0} \\
b^{1} \mathrm{G}_{4}-x^{1} \mathrm{H}_{5}^{5} \\
a^{1} \mathbf{F}_{3}-w^{1} \mathrm{D}_{2}^{2} \\
b^{3} \mathrm{H}_{4}-w^{1} \mathrm{G}_{4}^{2} \\
a^{5} \mathrm{D}_{2}-z^{3} \mathrm{~S}_{1}^{2} ?\end{array}$ & & \\
\hline $\begin{array}{l}1919.35 \\
1917.79 \\
1915.71 \\
1914.91 \\
1913.70\end{array}$ & $\begin{array}{l}0 \\
8\end{array}$ & $\begin{array}{l}20 \\
15 \\
15\end{array}$ tr & & $\begin{array}{l}52101.0 \\
52143.4 \\
52200.0 \\
52221.8 \\
52254.8\end{array}$ & $\begin{array}{l}a^{5} \mathrm{P}_{3}-x{ }^{5} \mathrm{D}_{2}^{2} \\
a^{1} \mathrm{~F}_{3}-3 \\
a^{5} \mathrm{P}_{2}-x \\
a^{5} \mathrm{P}_{3}-x^{5} \mathrm{D}_{1}^{\circ}\end{array}$ & & \\
\hline $\begin{array}{l}1913.10 \\
1912.39 \\
1911.88 \\
1909.36 \\
1908.32\end{array}$ & $\begin{array}{r}2 \\
8 \\
6 \\
8 \\
8 \\
10\end{array}$ & $\begin{array}{l}20 \\
40 \\
40 \\
40 \\
40 h\end{array}$ & & $\begin{array}{l}52271.2 \\
52290.6 \\
52304.5 \\
52373.6 \\
52402.1\end{array}$ & $\begin{array}{l}a^{5} \mathrm{~S}_{1}-x^{5} \mathrm{D}_{0}^{0} \\
a^{5} \mathrm{P}_{2}-x^{5} \mathrm{D}_{2}^{2} \\
a^{5} \mathrm{P}_{1}-x^{5} \mathrm{D}_{1}^{1} \\
a^{5} \mathrm{P}_{1}-x^{5} \mathrm{D}_{2}^{0} \\
a^{5} \mathrm{P}_{2}-x^{5} \mathrm{D}_{3}^{2}\end{array}$ & & \\
\hline $\begin{array}{l}1907.79 \\
1904.54 \\
1903.86\end{array}$ & 15 & ${ }_{5 ?}^{50} t r$ & & $\begin{array}{l}52416.7 \\
52506.1 \\
52524.9\end{array}$ & $\begin{array}{l}a^{5} \mathrm{P}_{3}-x^{5} \mathrm{D}_{4}^{\circ} \\
b^{3} \mathrm{~F}_{2}-x^{5} \mathrm{D}_{3}^{0} \\
b^{3} \mathrm{G}_{4}-u^{3} \mathrm{D}_{3}^{5} ?\end{array}$ & & \\
\hline 1897.70 & & 1 & & 52695.4 & $\left\{\begin{array}{l}a^{3}{ }^{3} \mathrm{P}_{2}-v{ }^{3} \mathrm{D}_{3}^{0} \\
b^{3} \mathrm{~F}_{4}-x^{1} \mathrm{~F}_{3}^{0}\end{array}\right.$ & & \\
\hline 1885.90 & & 10 & & 53025.1 & $a^{1} \mathrm{G}_{4}-x^{1} \mathrm{H}_{5}^{\circ}$ & & \\
\hline $\begin{array}{l}1883.98 \\
1880.43 \\
1878.90 \\
1878.19 \\
1877.85\end{array}$ & 6 & $\begin{array}{l}20 \\
40 e ? \\
10 \\
0 \\
\\
\\
\end{array}$ & & $\begin{array}{l}53079.1 \\
53179.3 \\
53222.6 \\
53242.7 \\
53252.4\end{array}$ & $\begin{array}{l}a^{3} \mathrm{~F}_{4}-w^{3} \mathrm{~F}_{4}^{9} \\
b^{1} \mathrm{G}_{4}-w^{1} \mathrm{G}_{4} \\
b^{3} \mathrm{G}_{4}-v^{3} \mathrm{G}_{3}^{3} \\
a^{3} \mathrm{~F}_{3}-w^{3} \mathrm{~F}_{2}^{2} \\
a^{1} \mathrm{H}_{5}-u^{3} \mathrm{~F}_{4}^{0}\end{array}$ & & \\
\hline $\begin{array}{l}1877.00 \\
1876.47 \\
1876.06 \\
1874.97 \\
1874.45\end{array}$ & $\begin{array}{l}3 \\
3\end{array}$ & $\begin{array}{r}10 \\
10 \\
20 \\
1 \\
12\end{array}$ & & $\begin{array}{l}53276.5 \\
53291.6 \\
53303.2 \\
53334.2 \\
53349.0\end{array}$ & $\begin{array}{l}a^{3} \mathrm{G}_{3}-v^{3} \mathrm{~F}_{2}^{\circ} \\
a^{3} \mathrm{~F}_{3}-w^{3} \mathrm{~F}_{3}^{3} \\
b^{3} \mathrm{G}_{3}-v^{3} \mathrm{G}_{3}^{3} \\
a^{3} \mathrm{~F}_{3}-w^{3} \mathrm{~F}_{4}^{8} \\
a^{3} \mathrm{G}_{4}-v^{3} \mathrm{~F}_{3}^{3}\end{array}$ & & \\
\hline $\begin{array}{l}\text { 1873. } 39 \\
1871.08\end{array}$ & 3 & $\begin{array}{l}10 \\
12\end{array}$ & & $\begin{array}{l}53379.2 \\
53445.1\end{array}$ & $\begin{array}{l}b^{3} \mathrm{G}_{5}-v^{3} \mathrm{G}_{4}^{2} \\
a^{3} \mathrm{~F}_{2}-w^{3} \mathrm{~F}_{2}\end{array}$ & & \\
\hline 1869.47 & 10 & 50 & & 53491.1 & $\left\{\begin{array}{l}a^{3} \mathrm{G}_{5}-v^{3} \mathrm{~F}_{4}^{0} \\
b^{3} \mathrm{H}_{4}-v^{3} \mathrm{G}_{4}^{0}\end{array}\right.$ & & \\
\hline $\begin{array}{l}1867.47 \\
1866.68\end{array}$ & & $\begin{array}{r}20 \\
5\end{array}$ & & $\begin{array}{l}53548.4 \\
53571.0\end{array}$ & $b^{3} \mathrm{G}_{3}-v^{3} \mathrm{G}_{4}^{\circ}$ & & \\
\hline $\begin{array}{l}\text { 1865. } 99 \\
1865.68 \\
1862.76 \\
1862.37 \\
1858.50\end{array}$ & 8 & $\begin{array}{l}3 \\
2 h d ? \\
25 \\
30 \\
2\end{array}$ & & $\begin{array}{l}53590.9 \\
53599.8 \\
53683.8 \\
53695.0 \\
53806.8\end{array}$ & $\begin{array}{l}a^{3} \mathrm{G}_{4}-v^{3} \mathrm{~F}_{4}^{\circ} \\
b^{1} \mathrm{D}_{2}-w^{1} \mathrm{D}_{2}^{2} \\
a^{1} \mathrm{D}_{2}-w^{1} \mathrm{~F}_{3}^{\circ} \\
b^{3} \mathrm{G}_{5}-v^{3} \mathrm{G}_{5}^{5} \\
b^{3} \mathrm{G}_{4}-v^{3} \mathrm{G}_{5}^{\circ}\end{array}$ & & \\
\hline $\begin{array}{l}1848.80 \\
1843.43 \\
1842.68 \\
1839.54 \\
1838.86\end{array}$ & & $\begin{array}{c}0 B l \text { FeII? } \\
5 \\
0 h \\
20 \\
25\end{array}$ & & $\begin{array}{l}54089.1 \\
54246.7 \\
54268.8 \\
54361.4 \\
54381.5\end{array}$ & $\begin{array}{l}a^{3} \mathrm{P}_{2}-x^{5} \mathrm{D}_{3}^{\circ} \\
b^{3} \mathrm{~F}_{2}-v^{3} \mathrm{~F}_{2}^{0} \\
a^{3} \mathrm{P}_{1}-x^{5} \mathrm{D}_{0}^{0} \\
b^{3} \mathrm{~F}_{3}-v^{3} \mathrm{~F}_{3}^{3} \\
a^{1} \mathrm{G}_{4}-w^{1} \mathrm{G}_{4}\end{array}$ & & \\
\hline
\end{tabular}

See footnotes at end of table. 
TABLE 1.-Second spectrum of Vanadium-Continued

\begin{tabular}{|c|c|c|c|c|c|c|c|}
\hline \multirow{3}{*}{$\lambda_{\mathrm{air}} \mathrm{A}$} & \multicolumn{2}{|r|}{2} & 3 & 4 & 5 & & \\
\hline & & tensity & \multirow{2}{*}{$\begin{array}{l}\text { Temp. } \\
\text { class }\end{array}$} & \multirow{2}{*}{$\begin{array}{l}\text { Wave } \\
\text { No. vac }\end{array}$} & \multirow{2}{*}{$\begin{array}{c}\text { Term } \\
\text { combination }\end{array}$} & \multicolumn{2}{|c|}{ Zeeman effect } \\
\hline & Arc & Spark & & & & Observed & Computed \\
\hline $\begin{array}{l}1837.76 \\
1833.58 \\
1828.84 \\
1823.61 \\
1817.09\end{array}$ & & $\begin{array}{ll} & t r \\
10 & \\
50 & \\
25 & \\
& t r\end{array}$ & & $\begin{array}{l}54414.1 \\
54538.1 \\
54679.5 \\
54836.3 \\
55033.0\end{array}$ & $\begin{array}{l}b^{3} \mathrm{~F}_{2}-v^{3} \mathrm{~F}_{3}^{\circ} \\
b^{3} \mathrm{~F}_{4}-v^{3} \mathrm{~F}_{4}^{0} \\
a^{3} F_{4}-w^{3} \mathrm{G}_{4}^{\circ}\end{array}$ & & \\
\hline $\begin{array}{l}1816.30 \\
1815.32 \\
1814.93 \\
1813.87\end{array}$ & & $\begin{array}{l}20 h \\
2 h d ? \\
5 \\
8\end{array}$ & & $\begin{array}{l}55057.0 \\
55086.7 \\
55098.5 \\
55130.7\end{array}$ & $\begin{array}{l}a^{3} \mathrm{D}_{4}-v^{3} \mathrm{G}_{3}^{0} ? \\
b^{3} \mathrm{G}_{2}-t^{3} \mathrm{D}_{1}^{0} \\
a^{3} \mathrm{~F}_{4}-w^{3} \mathrm{G}_{5}^{8}\end{array}$ & & \\
\hline 1811.51 & & $t r$ & & 55202.6 & 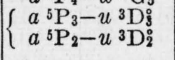 & & \\
\hline $\begin{array}{l}1810.57 \\
1810.31\end{array}$ & & ${ }^{3 e}$ tr & & $\begin{array}{l}\text { 55231. } 2 \\
55239.2\end{array}$ & $\begin{array}{l}b^{3} \mathrm{D}_{2}-t^{3} \mathrm{D}_{3}^{8} ? \\
a^{1} \mathrm{D}_{2}-u^{3} \mathrm{~F}_{2}^{3}\end{array}$ & & \\
\hline 1809.81 & & $8 d ?$ & & 55254.4 & $\left\{\begin{array}{l}b^{3} \mathrm{~F}_{3}-u^{3} \mathrm{D}_{2}^{0} \\
a^{3} \mathrm{G}_{5}-v^{3} \mathrm{G}_{4}^{4}\end{array}\right.$ & & \\
\hline $\begin{array}{l}1809.36 \\
1808.66\end{array}$ & & $\begin{array}{c}10 e \\
0\end{array}$ & & $\begin{array}{l}55268.2 \\
55289.6\end{array}$ & $\begin{array}{l}b^{3} \mathrm{~F}_{2}-u^{3} \mathrm{D}_{1}^{0} \\
a^{3} \mathrm{~F}_{3}-w^{3} \mathrm{G}_{4}^{0}\end{array}$ & & \\
\hline $\begin{array}{l}1807.15 \\
1806.49\end{array}$ & & $\begin{array}{l}1 \\
8 h\end{array}$ & & $\begin{array}{l}55335.7 \\
55356.0\end{array}$ & $\begin{array}{l}b^{3} \mathrm{~F}_{4}-u^{3} \mathrm{D}_{3}^{\circ} \\
a^{3} \mathrm{G}_{4}-v^{3} \mathrm{G}_{4}\end{array}$ & & \\
\hline 1804.53 & & $0 h$ & & 55416.1 & $\left\{\begin{array}{l}a^{3}{ }^{3} \mathrm{~F}_{2}-w \\
c^{3} \mathrm{P}_{2}{ }^{3} \mathrm{G}_{3}^{\circ} \\
{ }^{3} \mathrm{D}_{2}^{3}\end{array}\right.$ & & \\
\hline 1801.61 & & 0 & & 55505.9 & $\left\{\begin{array}{l}a^{3} \mathrm{~F}_{4}-v^{3} \mathrm{D}_{3}^{0} \\
c^{3} \mathrm{P}_{2}-t{ }^{3} \mathrm{D}_{3}^{3}\end{array}\right.$ & & \\
\hline 1800.13 & & $0 h$ & & 55551.5 & $b^{1} \mathrm{G}_{4}-w^{1} \mathrm{~F}_{3}^{\circ}$ & & \\
\hline $\begin{array}{l}1799.97 \\
179.47 \\
1796.80 \\
1796.52 \\
1796.26\end{array}$ & & $\begin{array}{c}3 \\
12 \\
20 \\
3 h \\
0\end{array}$ & & $\begin{array}{l}55556.5 \\
55571.9 \\
55654.5 \\
55663.2 \\
55671.2\end{array}$ & $\begin{array}{l}a{ }^{5} \mathrm{D}_{2}-y^{3} \mathrm{~S}_{1}^{\circ} \\
a^{3} \mathrm{G}_{5}-v^{3} \mathrm{G}_{5}^{\circ} \\
a_{5} \mathrm{D}_{0}-y^{3}{ }^{3} \mathrm{~S}_{1}^{\circ} \\
a^{3} \mathrm{G}_{4}-v^{3} \mathrm{G}_{5}^{3}\end{array}$ & & \\
\hline $\begin{array}{l}1795.38 \\
1794.62 \\
1793.13 \\
1792.49 \\
1789.61\end{array}$ & & $\begin{array}{c}0 \\
50 \\
3 \\
5 \\
5 h\end{array}$ & & $\begin{array}{l}55698.5 \\
55722.1 \\
55768.4 \\
55788.3 \\
55878.1\end{array}$ & $\begin{array}{l}b^{3} \mathrm{D}_{1}-u^{3} \mathrm{~F}_{2}^{2} \\
b^{3} \mathrm{D}_{2}-u^{3} \mathrm{~F}_{3}^{3} \\
b^{3} \mathrm{D}_{2}-23 \\
c^{3} \mathrm{P}_{2}-u^{3} \mathrm{~F}_{2} ?\end{array}$ & & \\
\hline $\begin{array}{l}1788.30 \\
1786.91 \\
1785.07\end{array}$ & & $\begin{array}{r}25 \\
0 \\
5\end{array}$ & & $\begin{array}{l}55919.0 \\
55962.5 \\
56020.2\end{array}$ & $\begin{array}{l}a^{3} \mathrm{~F}_{3}-v^{3} \mathrm{D}_{2}^{\circ} \\
b^{3} \mathrm{D}_{3}-u^{8} \mathrm{~F}_{4}^{0}\end{array}$ & & \\
\hline 1780.52 & & $5 h$ & & 56163.4 & $\left\{\begin{array}{l}a^{3} \mathrm{~F}_{2}-v^{3} \mathrm{D}_{2}^{0} \\
b^{3} \mathrm{H}_{4}-22_{3}^{\circ}\end{array}\right.$ & & \\
\hline 1776.48 & & $t r ?$ & & 56291.1 & $a^{3} \mathrm{~F}_{2}-v^{3} \mathrm{Di}_{\mathrm{i}}$ & & \\
\hline $\begin{array}{l}1773.00 \\
1767.02 \\
1766.21 \\
1763.20 \\
1760.11\end{array}$ & & $\begin{array}{rr} & t r \\
0 & \\
0 & \\
0 & \\
25 & \end{array}$ & & $\begin{array}{l}56401.6 \\
56592.5 \\
56618.4 \\
56715.1 \\
56814.6\end{array}$ & $\begin{array}{l}b^{3} \mathrm{H}_{4}-u^{3} \mathrm{~F}_{4}^{0} \\
b^{3} \mathrm{P}_{1}-t^{3} \mathrm{D}_{2}^{0} \\
b^{3} \mathrm{~F}_{4}-v^{3} \mathrm{G}_{5}^{0} \\
b^{3} \mathrm{P}_{2}-t^{3} \mathrm{D}_{3}^{0}\end{array}$ & & \\
\hline $\begin{array}{l}1759.00 \\
1757.76 \\
1748.99 \\
1739.33 \\
1737.02\end{array}$ & & $\begin{array}{c}{ } \\
20 \\
5 \\
10 l \\
0 H\end{array}$ & & $\begin{array}{l}56850.5 \\
56890.6 \\
57175.9 \\
57493.4 \\
57569.9\end{array}$ & $\begin{array}{l}a^{3} \mathrm{P}_{2}-u^{3} \mathrm{D}^{\mathrm{i}} \\
a^{3} \mathrm{~F}_{2}-x^{5} \mathrm{D}^{\mathrm{i}} \\
a^{3} \mathrm{D}_{3}-t^{3} \mathrm{D}_{3}^{\circ} ? \\
a^{3} \mathrm{~F}_{4}-y^{3} \mathrm{G}_{3}^{8} ?\end{array}$ & & \\
\hline $\begin{array}{l}1729.78 \\
1722.62 \\
1716.50 \\
1715.57 \\
1711.66\end{array}$ & & $\begin{array}{c}10 e \\
10 e \\
0 \\
2 \\
0\end{array}$ & & $\begin{array}{l}57810.8 \\
58051.1 \\
58258.1 \\
58289.7 \\
58422.8\end{array}$ & $\begin{array}{l}a{ }^{1} \mathrm{D}_{2}-w{ }^{1} \mathrm{D}_{2} \\
a{ }^{3} \mathrm{D}_{3}-2_{3}^{\circ} \\
a^{3} \mathrm{~F}_{4}-y^{5} \mathrm{G}_{5}^{\circ} ? \\
a^{3} \mathrm{D}_{3}-u^{3} \mathrm{~F}_{4}^{\circ} \\
b^{3} \mathrm{D}_{2}-3_{3}^{\circ}\end{array}$ & & \\
\hline $\begin{array}{l}1700.47 \\
1697.90 \\
1693.49 \\
1693.09 \\
1692.11\end{array}$ & & $\begin{array}{r}3 \\
2 \\
12 \\
10 \\
10\end{array}$ & & $\begin{array}{l}58807.3 \\
58896.3 \\
59049.7 \\
59063.6 \\
59097.8\end{array}$ & $\begin{array}{l}a^{3} \mathrm{~F}_{4}-v^{3} \mathrm{~F}_{3}^{0} \\
a^{3} \mathrm{~F}_{3}-v^{3} \mathrm{~F}_{2}^{0} \\
a^{3} \mathrm{~F}_{4}-v^{3} \mathrm{~F}_{4}^{0} \\
a^{3} \mathrm{~F}_{3}-v^{3} \mathrm{~F}_{3}^{0} \\
a^{3} \mathrm{~F}_{2}-v^{3} \mathrm{~F}_{2}^{\circ}\end{array}$ & & \\
\hline $\begin{array}{l}1687.36 \\
1686.19 \\
1682.17 \\
1681.22 \\
1677.88\end{array}$ & & $\begin{array}{l}1 h \\
1 \\
0 \\
5 \\
3\end{array}$ & & $\begin{array}{l}59264.2 \\
59305.3 \\
59447.0 \\
59480.6 \\
59599.0\end{array}$ & $\begin{array}{l}a{ }^{3} \mathrm{~F}_{2}-v^{3} \mathrm{~F}_{3}^{\circ} \\
a^{3} \mathrm{~F}_{3}-v^{3} \mathrm{~F}_{4}^{\circ} \\
a^{5} \mathrm{~F}_{2}-w^{3} \mathrm{~F}_{3}^{\circ} \\
a^{5} \mathrm{~F}_{1}-w^{3} \mathrm{~F}_{2}^{\circ} \\
a^{5} \mathrm{~F}_{5}-19\end{array}$ & & \\
\hline
\end{tabular}

See footnotes at end of table. 
TABLE 1.-Second spectrum of Vanadium-Continued

\begin{tabular}{|c|c|c|c|c|c|c|c|}
\hline 1 & & 2 & 3 & 4 & 5 & & \\
\hline \multirow{2}{*}{$\lambda_{\text {air }} \mathrm{A}$} & \multicolumn{2}{|c|}{ Intensity } & \multirow{2}{*}{$\begin{array}{l}\text { Temp. } \\
\text { class }\end{array}$} & \multirow{2}{*}{$\begin{array}{l}\text { Wave } \\
\text { No. vac }\end{array}$} & \multirow{2}{*}{$\begin{array}{c}\text { Term } \\
\text { combination }\end{array}$} & \multicolumn{2}{|c|}{ Zeeman effect } \\
\hline & Arc & Spark & & & & Observed & Computed \\
\hline $\begin{array}{l}1674.09 \\
1672.44 \\
1670.90 \\
1670.01 \\
1667.88\end{array}$ & & $\begin{array}{r}8 \\
15 \\
5 \\
10 \\
5\end{array}$ & & $\begin{array}{l}59733.9 \\
59792.9 \\
59848.0 \\
59879.9 \\
59956.4\end{array}$ & $\begin{array}{l}a^{5} \mathrm{~F}_{4}-1_{4}^{\circ} \\
a^{3} \mathrm{~F}_{4}-u^{3} \mathrm{D}_{3}^{\circ} \\
b^{3} \mathrm{G}_{3}-u^{3} \mathrm{~F}_{2}^{0} \\
a^{3} \mathrm{~F}_{3}-u^{3} \mathrm{D}_{2}^{\circ}\end{array}$ & & \\
\hline $\begin{array}{l}1667.66 \\
1665.42 \\
1664.87 \\
1663.80 \\
1663.60\end{array}$ & & $\begin{array}{c}10 \\
8 e \\
0 \\
0 \\
15\end{array}$ & & $\begin{array}{l}59964.3 \\
60044.9 \\
60064.7 \\
60103.4 \\
60110.6\end{array}$ & $\begin{array}{l}b^{3} \mathrm{G}_{4}-u^{3} \mathrm{~F}_{3}^{\circ} \\
b^{3} \mathrm{G}_{3}-u^{3} \mathrm{~F}_{3}^{\circ} \\
b^{3} \mathrm{G}_{3}-2_{3}^{\circ} \\
a^{3} \mathrm{~F}_{3}-u^{3} \mathrm{D}_{3}^{3} \\
b^{3} \mathrm{G}_{5}-u^{3} \mathrm{~F}_{4}^{\circ}\end{array}$ & & \\
\hline $\begin{array}{l}1663.34 \\
1662.28 \\
1661.27 \\
1660.53\end{array}$ & & $\begin{array}{r}3 \\
0 \\
60 \\
8\end{array}$ & & $\begin{array}{l}60120.0 \\
60158.3 \\
60194.9 \\
60221.7\end{array}$ & $\begin{array}{l}a^{3} \mathrm{~F}_{2}-u^{3}{ }^{3} \mathrm{D}_{1}^{\mathrm{i}} \\
a^{3} \mathrm{~F}_{2}-u^{3} \mathrm{D}_{2}^{2} \\
b^{3} \mathrm{G}_{4}-u^{3} \mathrm{~B}_{4}^{3}\end{array}$ & & \\
\hline 1643.43 & & 30 & & 60848.3 & $\left\{\begin{array}{l}a^{5} \mathrm{~F}_{4}-y^{5} \mathrm{~F}_{3}^{0} \\
a^{5} \mathrm{~F}_{3}-y^{5} \mathrm{~F}_{2}^{\circ}\end{array}\right.$ & & \\
\hline 1643.02 & & 30 & & 60863.5 & $\left\{\begin{array}{l}a^{5}{ }^{5} \mathrm{~F}_{3}-y^{8} \mathrm{~F}_{4}^{\circ} \\
a^{5} \mathrm{~F}_{2}-y^{5} \mathrm{~F}_{1}^{\circ}\end{array}\right.$ & & \\
\hline $\begin{array}{l}1640.86 \\
1640.15 \\
1639.13 \\
1637.93\end{array}$ & & $\begin{array}{l}30 \\
35 \\
40 \\
10\end{array}$ & & $\begin{array}{l}60943.7 \\
60970.0 \\
61008.0 \\
61052.7\end{array}$ & $\begin{array}{l}a^{5} \mathrm{~F}_{1}-y{ }^{5} \mathrm{~F}_{1}^{2} \\
a^{5} \mathrm{~F}_{2}-y{ }^{5} \mathrm{~F}_{2} \\
a^{5} \mathrm{~F}_{3}-y{ }^{5} \mathrm{~F}_{2} \\
a^{5} \mathrm{~F}_{1}-y{ }^{5} \mathrm{~F}_{2}^{2}\end{array}$ & & \\
\hline $\begin{array}{l}1637.77 \\
1637.55 \\
1636.02 \\
1635.86 \\
1634.98\end{array}$ & & $\begin{array}{r}50 \\
5 \\
40 \\
20 \\
t r\end{array}$ & & $\begin{array}{l}61058.6 \\
61066.8 \\
61123.9 \\
61129.9 \\
61162.8\end{array}$ & 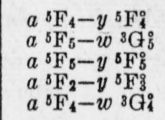 & & \\
\hline $\begin{array}{l}1633.51 \\
1630.82 \\
1619.18 \\
1617.35 \\
1613.40\end{array}$ & & $\begin{array}{r}25 \\
20 \\
10 \\
10 \\
0\end{array}$ & & $\begin{array}{l}61217.9 \\
61318.8 \\
61759.7 \\
61829.5 \\
61980.9\end{array}$ & $\begin{array}{l}a{ }^{5} \mathrm{~F}_{3}-y \\
a^{5} \mathrm{~s}^{5} \mathrm{~F}_{4}^{0} \\
a^{3} \mathrm{~F}_{4}-y \\
a^{3} \mathrm{G}_{3}-u^{3} \mathrm{~F}_{2}^{0} \\
a^{3} \mathrm{G}_{4}-u^{3} \mathrm{~F}_{3}^{0} \\
a^{5} \mathrm{~F}_{1}-x{ }^{1} \mathrm{D}_{2}^{2}\end{array}$ & & . \\
\hline $\begin{array}{l}1613.20 \\
1587.40 \\
1586.58 \\
1585.361 \\
1584.06\end{array}$ & & $\begin{array}{l}15 \\
50 \\
40 \\
30 \\
15\end{array}$ & & $\begin{array}{l}61988.6 \\
62996.1 \\
63028.7 \\
63077.1 \\
63128.9\end{array}$ & $\begin{array}{l}a^{3} \mathrm{G}_{5}-u^{3} \mathrm{~F}_{4}^{0} \\
a^{5} \mathrm{~F}_{5}-x^{5} \mathrm{D}^{2} \\
a^{5} \mathrm{~F}_{4}-x^{5} \mathrm{D}_{3}^{3} \\
a^{5} \mathrm{~F}_{3}-x^{5} \mathrm{D}_{2}^{2} \\
a^{5} \mathrm{~F}_{2}-x^{5} \mathrm{D}_{1}^{\circ}\end{array}$ & & \\
\hline 1582.80 & & 8 & & 63179.2 & $a^{5} \mathrm{~F}_{1}-x^{8} \mathrm{D}_{0}^{0}$ & & \\
\hline 1582.57 & & 15 & & 63188.4 & $a^{5} \mathrm{~F}_{3}-x^{5} \mathrm{D}_{3}^{0}$ & & \\
\hline $\begin{array}{l}1582.32 \\
1581.99 \\
1573.78\end{array}$ & & $\begin{array}{l}8 \\
8 \\
0\end{array}$ & & $\begin{array}{l}63198.3 \\
63211.5 \\
63541.3\end{array}$ & $\begin{array}{l}a^{5}{ }^{5} \mathrm{~F}_{2}-x^{5} \mathrm{D}_{2}^{\circ} \\
a^{5} \mathrm{~F}_{1}-x^{5} \mathrm{D}_{1}^{0} \\
a^{5} \mathrm{~F}_{2}-y^{5} \mathrm{G}_{2}^{8} ?\end{array}$ & & \\
\hline $\begin{array}{l}1571.74 \\
1565.98 \\
1562.98 \\
1558.76 \\
1556.05\end{array}$ & & $\begin{array}{r}2 \\
8 \\
10 \\
15 \\
0\end{array}$ & & $\begin{array}{l}63623.8 \\
63857.8 \\
63980.3 \\
64153.6 \\
64265.3\end{array}$ & $\begin{array}{l}a^{5} \mathrm{~F}_{1}-y^{5} \mathrm{G}_{2}^{2} ? \\
a^{5} \mathrm{~F}_{3}-y^{5} \mathrm{G}_{3}^{?} ? \\
a^{5} \mathrm{~F}_{2}-y^{5} \mathrm{G}_{3}^{3} ? \\
a^{5} \mathrm{~F}_{3}-y^{5} \mathrm{G}_{4}^{2} ? \\
a^{5} \mathrm{D}_{4}-v^{3} \mathrm{D}_{3}^{3}\end{array}$ & & \\
\hline 1553.09 & & 15 & & 64387.8 & 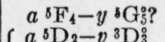 & & \\
\hline 1550.47 & & 0 & & 64496.6 & & & \\
\hline $\begin{array}{l}1547.20 \\
1358.40 \\
1313.82\end{array}$ & & $\begin{array}{r}15 \\
3 \\
0\end{array}$ & & $\begin{array}{l}64632.9 \\
73616.0 \\
76113.9\end{array}$ & $\begin{array}{l}a^{5} \mathrm{~F}_{5}-y^{5} \mathrm{G}_{6} ? \\
a^{5} \mathrm{~F}_{1}-u^{3} \mathrm{~F}_{2}^{2} \\
a^{5} \mathrm{D}_{2}-u^{3} \mathrm{~F}_{2}^{2}\end{array}$ & & \\
\hline
\end{tabular}

s Blend with VI.

b Zeeman pattern diffuse.

- Violet member of observed Zeeman pattern double line.

d Observed Zeeman pattern possible blend with V I.

- Observed Zeeman pattern affected by superposed line.

' Red member of observed Zeeman pattern double line.

g Observed Zeeman pattern affected by reversal.

h Observed Zeeman pattern unsymmetrical; doubtful.

i Observed Zeeman pattern blended.

i Intensities in observed Zeeman pattern unsymmetrical.

k Multiplet designation discordant with observed Zeeman pattern.

1 Violet $n$-component of observed Zeeman pattern blended.

m Red $n$-component of observed Zeeman pattern weaker and blended.

$n$ Red $n$-component of observed Zeeman pattern weaker.

o $n$-component of observed Zeeman pattern blended.

* Line probably a blend. 
The total number of $V_{\text {II }}$ lines listed in table 1 is 1,700 . Of these, 1,456 , or 86 percent, have been classified as combinations of singlet, triplet, and quintet terms, as follows: 81 singlet, 780 triplet, 215 quintet, 210 singlet-triplet, 202 triplet-quintet, 8 singlet-quintet, and 18 miscellaneous levels, 53 doing double and 2 triple duty because of blends. In such a complex spectrum it is inevitable that some predictable lines are unobserved because of masking by coincident or adjacent lines of greater intensity. A list of 24 such lines is displayed in table 2, where successive columns show (1) the masking spectrum line, (2) its observed wavelength, (3) the difference in wavelength between observed masking and calculated masked line, (4) estimated intensities of observed lines and expected intensities of masked lines, (5) wave number of observed line, and (6) term combination of the masked line. Considering their closeness to strong lines, their relatively low intensity, and the general characteristics of spark lines at atmospheric pressure, the number of masked lines is not unduly large.

The average difference in wavelength between these masking and masked lines is $0.04 \mathrm{~A}$, so that resolving power of the order of 100,000 would separate them if they were of equal intensity and reasonably sharp. But when the lines are somewhat diffuse and the intensity ratios average 10 or more, it appears to be impracticable to distinguish them if their separations are less than about $0.2 \mathrm{~A}$.

TABLE 2.-Masking and masked V II lines

\begin{tabular}{|c|c|c|c|c|c|c|}
\hline \multirow{2}{*}{$\begin{array}{l}\text { Spec- } \\
\text { trum }\end{array}$} & \multirow{2}{*}{$\begin{array}{c}\text { Observed } \\
\lambda\end{array}$} & \multirow{2}{*}{$\begin{array}{r}\Delta \lambda \\
\mathrm{o}^{-c} \mathrm{c}\end{array}$} & \multicolumn{2}{|c|}{ Intensity } & \multirow{2}{*}{$\begin{array}{c}\text { Observed } \\
\text { wave } \\
\text { number }\end{array}$} & \multirow{2}{*}{$\begin{array}{l}\text { Term combi- } \\
\text { nation }\end{array}$} \\
\hline & & & Observed & Expected & & \\
\hline V I & 4875.462 & -0.03 & 40 & 5 & 20505.17 & $c^{3} \mathrm{D}_{3}-v{ }^{3} \mathrm{D}_{3}^{\circ}$ \\
\hline V I & 4577.173 & $\begin{array}{r}.04 \\
+\quad .04\end{array}$ & 40 & 15 & 21841.44 & $a^{3} \mathrm{D}_{3}-z^{3} \mathrm{~F}_{3}$ \\
\hline V II & 4205.080 & $\begin{array}{r}T .03 \\
+.03\end{array}$ & 250 & 15 & 23774. 09 & $a^{5} \mathrm{P}_{2}-2{ }^{5} \mathrm{D}_{2}^{\circ}$ \\
\hline V I & 3844.442 & -.04 & 20 & 10 & 26004. 23 & $b^{3} \mathrm{~F}_{4}-z^{3} \mathrm{G}_{5}^{0}$ \\
\hline V II & 3337.845 & +.08 & 200 & 20 & 29950.87 & $b^{3} \mathrm{D}_{1}-y^{3} \mathrm{D}_{1}^{0}$ \\
\hline V II & 3093.108 & -.05 & $2500 R$ & 150 & 32320.59 & $b^{3} \mathrm{G}_{5}-y^{3} \mathrm{G}_{5}^{0}$ \\
\hline V II & 2982.75 & +.05 & 40 & 3 & 33516. 36 & $d^{3} \mathrm{~F}_{4}-w^{3} \mathrm{G}_{4}^{0}$ \\
\hline V II & 2944. 568 & +.08 & 250 & 15 & 33950.94 & $a^{3} \mathrm{D}_{2}-x^{3} \mathrm{~F}_{2}^{\circ}$ \\
\hline V II & 2941. 372 & $\begin{array}{r}+.15 \\
+\quad .15\end{array}$ & 200 & 20 & 33987.83 & $d^{3} \mathrm{~F}_{4}-v{ }^{3} \mathrm{D}_{3}^{0}$ \\
\hline V II & 2892.650 & +.08 & $200 r$ & 8 & 34560.27 & $c^{3} \mathrm{P}_{0}-x^{3} \mathrm{P}_{1}$ \\
\hline V II & 2880.026 & +.06 & 150 & 9 & 34711.75 & $a^{3} \mathrm{G}_{4}-y^{3} \mathrm{~F}_{4}$ \\
\hline V II & 2810.272 & +.03 & 100 & 20 & 35573.29 & $c^{3} \mathrm{P}_{1}-y{ }^{3} \mathrm{Si}$ \\
\hline V II & 2803.469 & -.05 & 150 & 4 & 35659.61 & $b^{3} \mathrm{~F}_{3}-y^{3} \mathrm{~F}_{2}^{0}$ \\
\hline V II & 2798.755 & +.02 & 80 & 4 & 35719.67 & $b^{3} \mathrm{~F}_{2}-y{ }^{3} \mathrm{~F}_{3}^{0}$ \\
\hline V II & 2756.58 & -.02 & $20 H l$ & 3 & 36266.14 & $a^{5} \mathrm{~F}_{4}-z^{3} \mathrm{G}_{3}^{\circ}$ \\
\hline V II & 2682.875 & +.07 & 100 & 6 & 37262. 39 & $d^{3} \mathrm{~F}_{3}-v{ }^{3} \mathrm{~F}_{3}^{0}$ \\
\hline V II & 2657. 295 & +.04 & 10 & 3 & 37621.07 & $a^{\circ} \mathrm{F}_{3}-2{ }^{3} \mathrm{~F}_{4}^{\circ}$ \\
\hline V II & 2628.75 & .00 & $30 H$ & ? & 38029.56 & $z^{5} \mathrm{G}_{5}^{\circ}-e^{5} \mathrm{~F}_{4}$ \\
\hline V III & 2593.052 & .00 & $50 \mathrm{He}$ & 2 & 38553.08 & $z{ }^{5} \mathrm{G}_{2}^{2}-e^{5} \mathrm{G}_{3}$ \\
\hline V II & 2548. 686 & +.04 & 60 & 10 & 39224. 14 & $b^{3} \mathrm{~F}_{3}-x^{3} \mathrm{D}_{3}^{\circ}$ \\
\hline VI & 2420.115 & +.05 & $100 R$ & 10 & 41307.79 & $a^{3} \mathrm{P}_{0}-x^{3} \mathrm{D}_{\mathrm{i}}$ \\
\hline V III & 2382.468 & -.01 & $80 H e$ & 20 & 41960.47 & $a^{3} \mathrm{P}_{2}-w^{3} \mathrm{D}_{2}$ \\
\hline V III & 2216.033 & -.02 & $100 e$ & 50 & 45111.59 & $a^{3} \mathrm{~F}_{2}-w{ }^{3} \mathrm{D}_{1}^{0}$ \\
\hline V II & 2134.12 & $\overline{0} .04$ & 200 & 100 & 46842.9 & 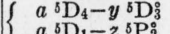 \\
\hline V II & 1797.64 & $\begin{array}{l}+.05 \\
-.04\end{array}$ & $15 N$ & $\begin{array}{r}50 \\
3\end{array}$ & 55628.5 & $\left(\begin{array}{ll}a \\
a\end{array}{ }^{5} D_{1}-y{ }^{3} S_{1}^{2}\right.$ \\
\hline
\end{tabular}

\section{TERMS OF THE VII SPECTRUM}

The established terms of the VIr spectrum are described in table 3, where singlet, triplet, and quintet terms are presented in groups. Successive columns show (1) the electron configuration responsible for the term, (2) term symbols, (3) level values, (4) level separations or intervals, (5) $g$-values derived from observed Zeeman effects, and 
(6) observed combinations. All level values are relative to $\left(3 d^{4}\right)$ $a^{5} \mathrm{D}_{0}=0.00$. The total number of levels is 223 , including 89 terms which are divided among multiplicities as follows: 31 singlet, 41 triplet, 17 quintet terms, and 3 miscellaneous levels. A large majority of the terms are regular, only 10 being wholly or partially inverted, as shown by negative signs in column 4. Landé's interval rules are reasonably well obeyed, as is shown by some examples in table 4; but $b^{3} \mathrm{P}, c{ }^{3} \mathrm{P}, b^{3} \mathrm{D}, c{ }^{3} \mathrm{~F}, d{ }^{3} \mathrm{~F}, z^{3} \mathrm{P}^{\circ}, y^{3} \mathrm{P}^{\circ}, x^{3} \mathrm{P}^{\circ}, x^{3} \mathrm{D}^{\circ}, w^{3} \mathrm{D}^{\circ}$, and $y^{3} \mathrm{~F}^{\circ}$ are exceptions, many of which exhibit partial inversion. Of special interest is $z^{5} \mathrm{D}^{\circ}$, whose first three intervals are law-abiding but the fourth is abnormally small, only $1 / 20$ of its expected value. These irregular intervals cannot be accounted for in any simple manner.

TABLE 3.-Terms of the V II spectrum*

\begin{tabular}{|c|c|c|c|c|c|}
\hline $\begin{array}{l}\text { Electron con- } \\
\text { figuration }\end{array}$ & $\begin{array}{l}\text { Term } \\
\text { symbol }\end{array}$ & Level & $\begin{array}{l}\text { Differ- } \\
\text { ence }\end{array}$ & $\begin{array}{c}\text { Ob- } \\
\text { served } \\
g\end{array}$ & Combinations \\
\hline $3 d^{4}$ & $a{ }^{1} \mathrm{~S}_{0}$ & 19902.60 & & & 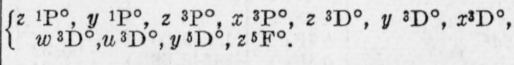 \\
\hline $3 d^{3} 48$ & $a^{1} \mathrm{P}_{1}$ & 22273.54 & & 0.97 & $\left\{\begin{array}{c}z^{1} \mathrm{~S}^{\circ}, z^{1} \mathrm{P}^{\circ}, y^{1} \mathrm{P}^{\circ}, z^{1} \mathrm{D}^{\circ}, y^{1} \mathrm{D}^{\circ}, x^{1} \mathrm{D}^{\circ}, y^{3} \mathrm{~S}^{\circ}, y^{3} \mathrm{P}^{\circ}, \\
x^{3} \mathrm{P}^{\circ}, y^{3} \mathrm{D}^{\circ}, x^{3} \mathrm{D}^{\circ}, y^{3} \mathrm{~F}^{\circ}, x^{3} \mathrm{~F}^{\circ}, y^{\circ} \mathrm{D}^{\circ} .\end{array}\right.$ \\
\hline $3 d^{4}$ & $a{ }^{1} \mathrm{D}_{2}$ & 20980.92 & & 1.02 & $\left\{\begin{array}{l}z^{1} \mathrm{P}^{\circ}, y^{1} \mathrm{P}^{\circ}, z{ }^{1} \mathrm{D}^{\circ}, x{ }^{1} \mathrm{D}^{\circ}, w^{1} \mathrm{D}^{\circ}, z{ }^{1} \mathrm{~F}^{\circ}, y{ }^{1} \mathrm{~F}^{\circ}, \\
x^{1} \mathrm{~F}^{\circ}, w^{1} \mathrm{~F}^{\circ}, z^{3} \mathrm{P}^{\circ}, y^{3} \mathrm{P}^{\circ}, x x^{3} \mathrm{P}^{\circ}, y^{3} \mathrm{D}^{\circ}, x^{3} \mathrm{D}^{\circ}, \\
w^{3} \mathrm{D}^{\circ}, z^{3} \mathrm{~F}^{\circ}, y^{3} \mathrm{~F}^{\circ}, x^{3} \mathrm{~F}^{\circ}, v^{3} \mathrm{~F}^{\circ}, u^{3} \mathrm{~F}^{\circ}, y^{2} \mathrm{G}^{\circ}, \\
v^{3} \mathrm{G}^{\circ} .\end{array}\right.$ \\
\hline $3 d^{3} 48$ & $b{ }^{1} \mathrm{D}_{2}$ & 25191.08 & & 0.99 & $\left\{\begin{array}{l}z^{1} \mathrm{P}^{\circ}, y{ }^{1} \mathrm{P}^{\circ}, z{ }^{1} \mathrm{D}^{\circ}, y^{1} \mathrm{D}^{\circ}, x{ }^{1} \mathrm{D}^{\circ}, w^{1}{ }^{1} \mathrm{D}^{\circ}, z^{1} \mathrm{~F}^{\circ}, \\
y^{1} \mathrm{~F}^{\circ}, x^{1} \mathrm{~F}^{\circ}, x^{3} \mathrm{P}^{\circ}, y^{3} \mathrm{D}^{\circ}, x^{3} \mathrm{D}^{\circ}, w^{3} \mathrm{D}^{\circ}, y^{3} \mathrm{~F}^{\circ},\end{array}\right.$ \\
\hline $3 d^{4}$ & $c{ }^{1} \mathrm{D}_{2}$ & 44657.99 & & & $x^{1} \mathrm{D}^{\circ}, x^{1} \mathrm{~F}^{\circ}$ \\
\hline $3 d^{4}$ & $a^{1} \mathrm{~F}_{3}$ & 26839.82 & & .97 & $\left\{\begin{array}{l}y{ }^{1} \mathrm{D}^{\circ}, x{ }^{1} \mathrm{D}^{\circ}, w{ }^{1} \mathrm{D}^{\circ}, z{ }^{1 \mathrm{~F}^{\circ}}, y^{1 \mathrm{~F}^{\circ}, w}{ }^{1 \mathrm{~F}^{\circ},}{ }^{y}{ }^{1}{ }^{1} \mathrm{G}^{\circ}, \\
y^{\circ},{ }^{3} \mathrm{D}^{\circ},{ }^{\circ} \mathrm{B}^{\circ}, 1^{\circ}, 3^{\circ},\end{array}\right.$ \\
\hline $3 d^{3} 4 s$ & $\mathrm{~b}^{1} \mathrm{~F}_{3}$ & 34228.79 & & 1.00 & $\left\{\begin{array}{l}{ }^{1} \mathrm{D}^{\circ}, y{ }^{1} \mathrm{D}^{\circ}, x{ }^{1} \mathrm{D}^{\circ}, z^{1} \mathrm{~F}^{\circ}, x{ }^{1} \mathrm{~F}^{\circ}, y^{1} \mathrm{G}^{\circ}, x{ }^{1} \mathrm{G}^{\circ}, \\
w^{1} \mathrm{G}^{\circ}, y^{3} \mathrm{~F}^{\circ}, w^{3} \mathrm{G}^{\circ} .\end{array}\right.$ \\
\hline $3 d^{4}$ & $a^{1} \mathrm{G}_{4}$ & 17910.98 & & 0.95 & $\left\{\begin{array}{c}z^{1} \mathrm{~F}^{\circ}, y{ }^{1 \mathrm{~F}^{\circ}}, x{ }^{1} \mathrm{~F}^{\circ}, z^{1} \mathrm{G}^{\circ}, y{ }^{1} \mathrm{G}^{\circ}, x{ }^{1} \mathrm{G}^{\circ}, w{ }^{1} \mathrm{G}^{\circ}, \\
z^{1} \mathrm{H}^{\circ}, y^{1 \mathrm{H}^{\circ},} x^{1} \mathrm{H}^{\circ}, z^{3} \mathrm{~F}^{\circ}, y^{3} \mathrm{~F}^{\circ}, y^{3} \mathrm{G}^{\circ}, x^{3} \mathrm{G}^{\circ}, \\
v^{3} \mathrm{G}^{\circ}, z^{3} \mathrm{H}^{\circ}, y^{3} \mathrm{H}^{\circ}, y^{5} \mathrm{~F}^{\circ} .\end{array}\right.$ \\
\hline $3 d^{3} 4 s$ & $b{ }^{1} \mathrm{G}_{4}$ & 19112.93 & & .98 & $\left\{\begin{array}{c}z^{1} \mathrm{~F}^{\circ}, y{ }^{1} \mathrm{~F}^{\circ}, x{ }^{1} \mathrm{~F}^{\circ}, w{ }^{1} \mathrm{~F}^{\circ}, z{ }^{1} \mathrm{G}^{\circ}, y{ }^{1} \mathrm{G}^{\circ}, x{ }^{1} \mathrm{G}^{\circ}, \\
w^{1} \mathrm{G}^{\circ}, z^{1} \mathrm{H}^{\circ}, y^{1} \mathrm{H}^{\circ}, x{ }^{1} \mathrm{H}^{\circ}, w^{3} \mathrm{D}^{\circ}, u^{3} \mathrm{D}^{\circ}, y^{3} \mathrm{~F}^{\circ}, \\
z^{3} \mathrm{G}^{\circ}, y^{3} \mathrm{G}^{\circ}, x^{3} \mathrm{G}^{\circ}, v^{3} \mathrm{G}^{\circ}, z^{3} \mathrm{H}^{\circ}, y^{3} \mathrm{H}^{\circ}, z^{3} \mathrm{I}^{\circ} .\end{array}\right.$ \\
\hline $3 d^{4}$ & $c{ }^{1} \mathrm{G}_{4}$ & 36425.07 & & .96 & $y^{1} \mathrm{~F}^{\circ}, x^{1} \mathrm{~F}^{\circ}, y^{1} \mathrm{G}^{\circ}, x^{1} \mathrm{G}^{\circ}, y^{1} \mathrm{H}^{\circ}, x^{3} \mathrm{G}^{\circ}, w^{3} \mathrm{G}^{\circ}$. \\
\hline $3 d^{3} 4 s$ & $a^{1} \mathrm{H}_{5}$ & 23391. 09 & & 1.04 & $\left\{\begin{array}{l}z^{1} \mathrm{G}^{\circ}, y^{1} \mathrm{G}^{\circ}, x^{1} \mathrm{G}^{\circ}, w{ }^{1} \mathrm{G}^{\circ}, z{ }^{1} \mathrm{H}^{\circ}, y{ }^{1} \mathrm{H}^{\circ}, z{ }^{1} \mathrm{I}^{\circ}, \\
x^{3} \mathrm{~F}^{\circ}, w^{3} \mathrm{~F}^{\circ}, u^{3} \mathrm{~F}^{\circ}, y^{3} \mathrm{G}^{\circ}, x^{3} \mathrm{G}^{\circ}, w^{3} \mathrm{G}^{\circ}, y^{3} \mathrm{H}^{\circ}, \\
z^{3} \mathrm{I}^{\circ}, 1^{\circ} .\end{array}\right.$ \\
\hline $3 d^{4}$ & $a{ }^{1} \mathrm{I}_{6}$ & 19191.50 & & $0.96:$ & $z^{1} \mathrm{H}^{\circ}, y^{1} \mathrm{H}^{\circ}, x^{1} \mathrm{H}^{\circ}, z^{1} \mathrm{I}^{\circ}, y^{3} \mathrm{G}^{\circ}, x^{3} \mathrm{G}^{\circ}$. \\
\hline $3 d^{4}$ & $\begin{array}{l}a^{3} \mathrm{P}_{2} \\
a^{3} \mathrm{P}_{1} \\
a^{3} \mathrm{P}_{0}\end{array}$ & $\begin{array}{l}11908.27 \\
11514.76 \\
11295.51\end{array}$ & $\begin{array}{l}393.51 \\
219.25\end{array}$ & $\begin{array}{l}1.49 \\
1.48\end{array}$ & $\left\{\begin{array}{l}z{ }^{3} \mathrm{~S}^{\circ}, y{ }^{3} \mathrm{~S}^{\circ}, z{ }^{3} \mathrm{P}^{\circ}, y{ }^{3} \mathrm{P}^{\circ}, x{ }^{3} \mathrm{P}^{\circ}, z{ }^{3} \mathrm{D}^{\circ}, y{ }^{3} \mathrm{D}^{\circ}, \\
x^{3} \mathrm{D}^{\circ}, w^{3} \mathrm{D}^{\circ}, v{ }^{3} \mathrm{D}^{\circ}, u^{3} \mathrm{D}^{\circ}, z^{3} \mathrm{~F}^{\circ}, x^{3} \mathrm{~F}^{\circ}, z \mathrm{~S}^{\circ}, \\
z^{1} \mathrm{P}^{\circ}, z^{1} \mathrm{D}^{\circ}, y^{1} \mathrm{~F}^{\circ}, z^{5} \mathrm{~S}^{\circ}, z^{5} \mathrm{D}^{\circ}, y^{\circ} \mathrm{D}^{\circ}, x{ }^{\circ} \mathrm{D}^{\circ}, \\
z^{5} \mathrm{~F}^{\circ}, 2^{\circ} .\end{array}\right.$ \\
\hline $3 d^{3} 4 s$ & $\begin{array}{l}b{ }^{3} \mathrm{P}_{2} \\
b^{3} \mathrm{P}_{1} \\
b^{3} \mathrm{P}_{0}\end{array}$ & $\begin{array}{l}19132.69 \\
19166.19 \\
19161.27\end{array}$ & $\begin{array}{r}-33.50 \\
4.92\end{array}$ & $\begin{array}{l}1.38 \\
1.40\end{array}$ & $\left\{\begin{array}{l}z^{3} \mathrm{~S}^{\circ}, z^{3} \mathrm{P}^{\circ}, y{ }^{3} \mathrm{P}^{\circ}, x{ }^{3} \mathrm{P}^{\circ}, y^{3} \mathrm{D}^{\circ}, x^{3} \mathrm{D}^{\circ}, w^{3} \mathrm{D}^{\circ}, \\
v^{3} \mathrm{D}^{\circ}, u^{3} \mathrm{D}^{\circ}, t^{3} \mathrm{D}^{\circ}, y^{3} \mathrm{~F}^{\circ}, x^{3} \mathrm{~F}^{\circ}, v^{3} \mathrm{~F}^{\circ}, y^{3} \mathrm{G}^{\circ}, \\
z^{1} \mathrm{~S}^{\circ}, z^{1} \mathrm{P}^{\circ}, z^{1} \mathrm{D}^{\circ}, y^{1} \mathrm{D}^{\circ}, y^{1} \mathrm{~F}^{\circ}, z^{3} \mathrm{P}^{\circ}, y^{5} \mathrm{D}^{\circ} .\end{array}\right.$ \\
\hline $3 d^{3} 48$ & $\begin{array}{l}c^{3} \mathrm{P}_{2} \\
c^{3} \mathrm{P}_{1} \\
c^{3} \mathrm{P}_{0}\end{array}$ & $\begin{array}{l}20343.00 \\
20089.56 \\
20156.64\end{array}$ & $\begin{array}{r}253.44 \\
-67.08\end{array}$ & $\begin{array}{l}1.36 \\
1.35\end{array}$ & $\left\{\begin{array}{l}z^{3} \mathrm{~S}^{\circ}, y^{3} \mathrm{~S}^{\circ}, z^{3} \mathrm{P}^{\circ}, y^{3} \mathrm{P}^{\circ}, x^{3} \mathrm{P}^{\circ}, z^{3} \mathrm{D}^{\circ}, y^{3} \mathrm{D}^{\circ}, x^{3} \mathrm{D}^{\circ}, \\
w^{3} \mathrm{D}^{\circ}, v^{3} \mathrm{D}^{\circ}, u^{3} \mathrm{D}^{\circ}, t^{3} \mathrm{D}^{\circ}, y^{3} \mathrm{~F}^{\circ}, x^{3} \mathrm{~F}^{\circ}, w^{3} \mathrm{~F}^{\circ}, \\
v^{3} \mathrm{~F}^{\circ}, u^{3} \mathrm{~F}^{\circ}, z^{1} \mathrm{~S}^{\circ}, z^{1} \mathrm{P}^{\circ}, z^{1} \mathrm{D}^{\circ}, x^{1} \mathrm{D}^{\circ}, z^{1} \mathrm{~F}^{\circ}, \\
y^{1} \mathrm{~F}^{\circ}, z^{5} \mathrm{~S}^{\circ}, z^{5} \mathrm{P}^{\circ}, z^{5} \mathrm{D}^{\circ}, y^{6} \mathrm{D}^{\circ}, z^{3} \mathrm{~F}^{\circ} .\end{array}\right.$ \\
\hline $3 d^{4}$ & $\begin{array}{l}d^{3} \mathrm{P}_{2} \\
d^{3} \mathrm{P}_{1} \\
d^{3} \mathrm{P}_{0}\end{array}$ & $\begin{array}{l}32040.76 \\
32299.24 \\
32420.04\end{array}$ & $\begin{array}{l}-258.48 \\
-120.80\end{array}$ & $\begin{array}{l}1.38 \\
1.48\end{array}$ & $\left\{\begin{array}{l}z^{3} \mathrm{~S}^{\circ}, y^{3} \mathrm{~S}^{\circ}, z^{3} \mathrm{P}^{\circ}, x^{3} \mathrm{P}^{\circ}, y^{3} \mathrm{D}^{\circ} \cdot x^{3} \mathrm{D}^{\circ}, v^{3} \mathrm{D}^{\circ}, u^{3} \mathrm{D}^{\circ}, \\
x^{3} \mathrm{~F}^{\circ}, y{ }^{1} \mathrm{P}^{\circ}, y^{1} \mathrm{D}^{\circ} .\end{array}\right.$ \\
\hline
\end{tabular}

* Colon denotes values are poorly determined. 
TABLE 3.-Terms of the V II spectrum-Continued

\begin{tabular}{|c|c|c|c|c|c|}
\hline $\begin{array}{l}\text { Electron con- } \\
\text { figuration }\end{array}$ & $\begin{array}{l}\text { Term } \\
\text { symbol }\end{array}$ & Level & $\begin{array}{l}\text { Differ- } \\
\text { ence }\end{array}$ & $\begin{array}{l}\text { Ob- } \\
\text { served } \\
g\end{array}$ & Combinations \\
\hline $3 d^{4}$ & $\begin{array}{l}a^{3} \mathrm{D}_{3} \\
a^{3} \mathrm{D}_{2} \\
a^{3} \mathrm{D}_{1}\end{array}$ & $\begin{array}{l}18353.89 \\
18293.87 \\
18269.49\end{array}$ & $\begin{array}{l}60.02 \\
24.38\end{array}$ & $\begin{array}{l}1.30 \\
1.13 \\
0.49\end{array}$ & 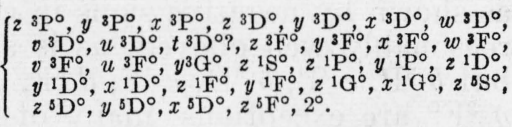 \\
\hline $3 d^{3} 4 s$ & $\begin{array}{l}b^{3} \mathrm{D}_{3} \\
b^{3} \mathrm{D}_{2} \\
b^{3} \mathrm{D}_{1}\end{array}$ & $\begin{array}{l}20622.99 \\
20617.05 \\
20522.14\end{array}$ & $\begin{array}{r}5.94 \\
-5.09\end{array}$ & $\begin{array}{l}1.26 \\
1.25 \\
0.58\end{array}$ & 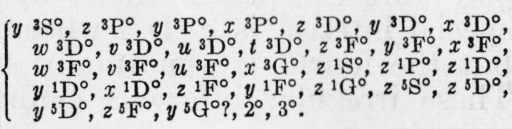 \\
\hline $3 d^{3} 4 s$ & $\begin{array}{l}c^{3} \mathrm{D}_{3} \\
c^{3} \mathrm{D}_{2} \\
c^{3} \mathrm{D}_{1}\end{array}$ & $\begin{array}{l}44098.46 \\
44159.43 \\
44200.97: ?\end{array}$ & $\begin{array}{l}-60.97 \\
-41.54\end{array}$ & $\begin{array}{l}(1.27:) \\
(1.14:) \\
(0.50:)\end{array}$ & $\left\{v^{3} \mathrm{D}^{\circ}, t{ }^{3} \mathrm{D}^{\circ}, w^{3} \mathrm{~F}^{\circ}, u^{3} \mathrm{~F}^{\circ}\right.$ \\
\hline $3 d^{3} 4 s$ & $\begin{array}{l}a^{3} \mathrm{~F}_{4} \\
a^{3} \mathrm{~F}_{3} \\
a^{3} \mathrm{~F}_{2}\end{array}$ & $\begin{array}{l}9097.81 \\
8841.97 \\
8640.21\end{array}$ & $\begin{array}{l}255.84 \\
201.76\end{array}$ & $\begin{array}{l}1.22 \\
1.04 \\
0.65\end{array}$ & 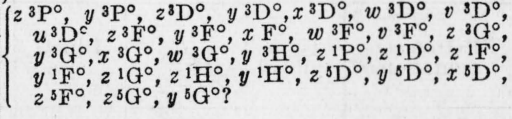 \\
\hline$s d^{4}$ & $\begin{array}{l}b{ }^{3} \mathrm{~F}_{4} \\
b \mathrm{~B}_{3} \\
b^{3} \mathrm{~F}_{3}\end{array}$ & $\begin{array}{l}13609.00 \\
13542.68 \\
13490.84\end{array}$ & $\begin{array}{l}66.32 \\
51.84\end{array}$ & $\begin{array}{l}1.19 \\
1.06 \\
0.59\end{array}$ & $\left\{\begin{array}{l}z^{3} \mathrm{D}^{\circ}, y^{3} \mathrm{D}^{\circ}, x^{3} \mathrm{D}^{\circ}, w^{3} \mathrm{D}^{\circ}, v{ }^{3} \mathrm{D}^{\circ}, u^{3} \mathrm{D}^{\circ}, z^{3} \mathrm{~F}^{\circ}, \\
y^{3} \mathrm{~F}^{\circ}, x^{3} \mathrm{~F}^{\circ}, w^{3} \mathrm{~F}^{\circ}, v^{3} \mathrm{~F}^{\circ}, z^{3} \mathrm{G}^{\circ}, y^{3} \mathrm{G}^{\circ}, x^{3} \mathrm{G}^{\circ}, \\
w^{3} \mathrm{G}^{\circ}, v^{3} \mathrm{G}^{\circ}, z^{3} \mathrm{H}^{\circ}, y^{3} \mathrm{H}^{\circ}, z^{1} \mathrm{P}^{\circ}, z^{1} \mathrm{D}^{\circ}, z^{1} \mathrm{~F}^{\circ}, \\
y{ }^{1} \mathrm{~F}^{\circ}, x^{1} \mathrm{~F}^{\circ}, z^{1} \mathrm{G}^{\circ}, z^{1} \mathrm{H}^{\circ}, y^{1} \mathrm{H}^{\circ}, z^{3} \mathrm{~S}^{\circ}, z^{5} \mathrm{D}^{\circ}, \\
x{ }^{\circ} \mathrm{D}^{\circ}, z^{3} \mathrm{~F}^{\circ}, z^{5} G^{\circ} .\end{array}\right.$ \\
\hline $3 d^{4}$ & $\begin{array}{l}c^{3} \mathrm{~F}_{4} \\
c \mathrm{~F}_{3}^{3} \\
c^{3} \mathrm{~F}_{2}\end{array}$ & $\begin{array}{l}30318.63 \\
30306.40 \\
30267.46\end{array}$ & $\begin{array}{l}12.23 \\
38.94\end{array}$ & $\begin{array}{l}1.25 \\
1.06 \\
0.67\end{array}$ & $\left\{\begin{array}{c}y^{3} \mathrm{D}^{\circ}, x^{3} \mathrm{D}^{\circ}, w^{3} \mathrm{D}^{\circ}, v^{3} \mathrm{D}^{\circ}, u^{3} \mathrm{D}^{\circ}, t{ }^{3} \mathrm{D}^{\circ}, y^{3} \mathrm{~F}^{\circ}, \\
x^{3} \mathrm{~F}^{\circ}, w^{3} \mathrm{~F}^{\circ}, v^{3} \mathrm{~F}^{\circ}, u^{3} \mathrm{~F}^{\circ}, x^{3} \mathrm{G}^{\circ}, w^{3} \mathrm{G}^{\circ}, v^{3} \mathrm{G}^{\circ}, \\
y^{1} \mathrm{D}^{\circ}, y^{1} \mathrm{~F}^{\circ}, z^{1} \mathrm{G}^{\circ}, y^{1} \mathrm{H}^{\circ}, x^{3} \mathrm{D}^{\circ}, y^{3} \mathrm{G}^{\circ} ? 2^{\circ}, 3^{\circ} .\end{array}\right.$ \\
\hline $3 d^{3} 4 s$ & $\begin{array}{l}d \mathrm{~F}_{4} \\
d^{3} \mathrm{~F}_{3} \\
d^{3} \mathrm{~F}_{2}\end{array}$ & $\begin{array}{l}30613.97 \\
30641.71 \\
30673.14\end{array}$ & $\begin{array}{l}-27.74 \\
-31.43\end{array}$ & $\begin{array}{l}1.23 \\
1.05 \\
0.67\end{array}$ & $\left\{\begin{array}{l}y^{3} \mathrm{D}^{\circ}, x^{3} \mathrm{D}^{\circ}, w^{3} \mathrm{D}^{\circ}, v^{3} \mathrm{D}^{\circ}, t{ }^{3} \mathrm{D}^{\circ}, x^{3} \mathrm{~F}^{\circ}, w^{3} \mathrm{~F}^{\circ}, \\
v^{3} \mathrm{~F}^{\circ}, u^{3} \mathrm{~F}^{\circ}, x^{3} \mathrm{G}^{\circ}, w^{3} \mathrm{G}^{\circ}, v^{3} \mathrm{G}^{\circ}, z^{3} \mathrm{H}^{\circ}, z^{1} \mathrm{P}^{\circ}, \\
z^{1} \mathrm{D}^{\circ}, w^{1} \mathrm{D}^{\circ}, z^{1} \mathrm{~F}^{\circ}, y^{1} \mathrm{~F}^{\circ}, w^{1} \mathrm{~F}^{\circ}, x^{1} \mathrm{G}^{\circ}, y^{1} \mathrm{H}^{\circ}, \\
y^{5} \mathrm{G}^{\circ} ? 2^{\circ}, 3^{\circ} .\end{array}\right.$ \\
\hline $3 d^{4}$ & $\begin{array}{l}a^{3} \mathrm{G}_{5} \\
a^{3} \mathrm{G}_{4} \\
a^{3} \mathrm{G}_{3}\end{array}$ & $\begin{array}{l}14655.63 \\
14556.09 \\
14461.73\end{array}$ & $\begin{array}{l}99.54 \\
94.36\end{array}$ & $\begin{array}{l}1.17 \\
1.00 \\
0.74\end{array}$ & $\left\{\begin{array}{c}y^{3} \mathrm{D}^{\circ}, x^{3} \mathrm{D}^{\circ}, w^{3} \mathrm{D}^{\circ}, z^{3} \mathrm{~F}^{\circ}, y^{3} \mathrm{~F}^{\circ}, x^{3} \mathrm{~F}^{\circ}, w^{3} \mathrm{~F}^{\circ}, \\
v^{3} \mathrm{~F}^{\circ}, u^{3} \mathrm{~F}^{\circ}, z^{3} \mathrm{G}^{\circ}, y^{3} \mathrm{G}^{\circ}, x^{3} \mathrm{G}^{\circ}, w^{3} \mathrm{G}^{\circ}, v^{3} \mathrm{G}^{\circ}, \\
z^{3} \mathrm{H}^{\circ}, y^{3} \mathrm{H}^{\circ}, z^{1} \mathrm{D}^{\circ}, x^{1} \mathrm{D}^{\circ}, z^{1} \mathrm{~F}^{\circ}, y^{1} \mathrm{~F}^{\circ}, z^{1} \mathrm{G}^{\circ}, \\
y^{1} \mathrm{G}^{\circ}, z^{1} \mathrm{H}^{\circ}, y^{1} \mathrm{H}^{\circ}, z^{5} \mathrm{D}^{\circ}, x^{3} \mathrm{D}^{\circ}, z^{3} \mathrm{~F}^{\circ}, y^{3} \mathrm{~F}^{\circ}\end{array}\right.$ \\
\hline $3 d^{3} 48$ & $\begin{array}{l}b^{3} \mathrm{G}_{3} \\
b^{3} \mathrm{G}_{4} \\
b^{3} \mathrm{G}_{3}\end{array}$ & $\begin{array}{l}16533.00 \\
16421.51 \\
16340.97\end{array}$ & $\begin{array}{r}111.49 \\
80.54\end{array}$ & $\begin{array}{l}1.16 \\
1.03 \\
0.76\end{array}$ & $\left\{\begin{array}{l}z^{3} \mathrm{D}^{\circ}, u^{3} \mathrm{D}^{\circ} ?, z^{3} \mathrm{~F}^{\circ}, y^{3} \mathrm{~F}^{\circ}, w^{3} \mathrm{~F}^{\circ}, v{ }^{3} \mathrm{~F}^{\circ}, u^{3} \mathrm{~F}^{\circ}, \\
z^{3} \mathrm{G}^{\circ}, y^{3} \mathrm{G}^{\circ}, x^{3} \mathrm{G}^{\circ}, w^{3} \mathrm{G}^{\circ}, v \mathrm{G}^{\circ}, z^{3} \mathrm{H}^{\circ}, y^{3} \mathrm{H}^{\circ}, \\
z^{1} \mathrm{D}^{\circ}, z^{1} \mathrm{~F}^{\circ}, y^{1} \mathrm{~F}^{\circ}, x^{1} \mathrm{~F}^{\circ}, z^{1} \mathrm{G}^{\circ}, y^{1} \mathrm{G}^{\circ}, z^{1} \mathrm{H}^{\circ}, \\
y^{1} \mathrm{H}^{\circ}, y^{5} \mathrm{G}^{\circ} ?, 2^{\circ} .\end{array}\right.$ \\
\hline $3 d^{4}$ & $\begin{array}{l}a^{3} \mathrm{H}_{6} \\
a^{3} \mathrm{H}_{3} \\
a^{3} \mathrm{H}_{4}\end{array}$ & $\begin{array}{l}12706.15 \\
12621.57 \\
12545.15\end{array}$ & $\begin{array}{l}84.58 \\
76.42\end{array}$ & $\begin{array}{l}\text { 1. } 27: \\
\text { 1. } 02 \\
0.83\end{array}$ & $\left\{\begin{array}{l}y^{3} \mathrm{~F}^{\circ}, x^{3} \mathrm{~F}^{\circ}, w^{3} \mathrm{~F}^{\circ}, z^{3} \mathrm{G}^{\circ}, y^{3} \mathrm{G}^{\circ}, x^{3} \mathrm{G}^{\circ}, w^{3} \mathrm{G}^{\circ}, \\
z^{3} \mathrm{H}^{\circ}, y^{3} \mathrm{H}^{\circ}, z^{3} \mathrm{I}^{\circ}, z^{1} \mathrm{~F}^{\circ}, y^{1} \mathrm{~F}^{\circ}, z^{1} \mathrm{G}^{\circ}, y^{1} \mathrm{G}^{\circ}, \\
z^{1} \mathrm{H}^{\circ}, y^{1} \mathrm{H}^{\circ}, z^{1} \mathrm{I}^{\circ}, z^{5} \mathrm{~F}^{\circ}, y^{3} \mathrm{~F}^{\circ} .\end{array}\right.$ \\
\hline $3 d^{3} 4 s$ & $\begin{array}{l}b{ }^{3} \mathrm{H}_{8} \\
b{ }^{3} \mathrm{H}_{5} \\
b^{3} \mathrm{H}_{4}\end{array}$ & $\begin{array}{l}20363.22 \\
20280.19 \\
20242.32\end{array}$ & $\begin{array}{l}83.03 \\
37.87\end{array}$ & $\begin{array}{l}1.14 \\
1.01 \\
0.82\end{array}$ & $\left\{\begin{array}{c}y^{3} \mathrm{~F}^{\circ}, u^{3} \mathrm{~F}^{\circ}, z^{3} \mathrm{G}^{\circ}, y^{3} \mathrm{G}^{\circ}, x^{3} \mathrm{G}^{\circ}, w^{3} \mathrm{G}^{\circ}, v^{3} \mathrm{G}^{\circ}, \\
z^{3} \mathrm{H}^{\circ}, y^{3} \mathrm{H}^{\circ}, z^{3} \mathrm{I}^{\circ}, z^{1} \mathrm{~F}^{\circ}, y^{1} \mathrm{~F}^{\circ}, z^{1} \mathrm{G}^{\circ}, y^{1} \mathrm{G}^{\circ}, \\
w^{1} \mathrm{G}^{\circ}, z^{1} \mathrm{H}^{\circ}, y^{1} \mathrm{H}^{\circ}, x^{1} \mathrm{H}^{\circ}, z^{1} \mathrm{I}^{\circ}, 2^{\circ} .\end{array}\right.$ \\
\hline $3 d^{3} 4 s$ & $\begin{array}{l}a{ }^{5} \mathrm{P}_{3} \\
a \\
a^{5} \mathrm{P}_{2} \\
5 \mathrm{P}_{1}\end{array}$ & $\begin{array}{l}13741.61 \\
13594.73 \\
13511.71\end{array}$ & $\begin{array}{r}146.88 \\
83.02\end{array}$ & $\begin{array}{l}1.62 \\
1.78 \\
2.39\end{array}$ & $\left\{\begin{array}{l}z^{5} \mathrm{~S}^{\circ}, z^{3} \mathrm{P}^{\circ}, z^{5} \mathrm{D}^{\circ}, y{ }^{3} \mathrm{D}^{\circ}, x^{5} \mathrm{D}^{\circ}, z^{5} \mathrm{~F}^{\circ}, z{ }^{1} \mathrm{P}^{\circ} \\
y{ }^{3} \mathrm{~S}^{\circ}, z^{3} \mathrm{P}^{\circ}, y^{3} \mathrm{P}^{\circ}, z^{3} \mathrm{D}^{\circ}, y^{3} \mathrm{D}^{\circ}, x^{3} \mathrm{D}^{\circ}, u^{3} \mathrm{D}^{\circ}, \\
1^{\circ} .\end{array}\right.$ \\
\hline $3 d^{4}$ & $\begin{array}{l}a \\
a \\
a \\
a^{5} \mathrm{~S}_{1} \mathrm{D}_{3} \\
a^{5} \mathrm{D}_{2} \\
a^{5} \mathrm{D}_{1} \\
a^{5} \mathrm{D}_{0}\end{array}$ & $\begin{array}{r}339.21 \\
208.89 \\
106.63 \\
36.05 \\
0.00\end{array}$ & $\begin{array}{r}130.32 \\
102.26 \\
70.58 \\
36.05\end{array}$ & & $\left\{\begin{array}{l}z^{5} \mathrm{P}^{\circ}, z^{2}{ }^{5} \mathrm{D}^{\circ}, y^{5} \mathrm{D}^{\circ}, z^{5} \mathrm{~F}^{\circ}, z^{3} \mathrm{~S}^{\circ} ?, y^{3} \mathrm{~S}^{\circ}, z^{3} \mathrm{P}^{\circ}, \\
z^{3} \mathrm{D}^{\circ}, v^{3} \mathrm{D}^{\circ}, z^{3} \mathrm{~F}^{\circ}, u^{3} \mathrm{~F}^{\circ}, z^{3} \mathrm{G}^{\circ} .\end{array}\right.$ \\
\hline $3 d^{3} 4 s$ & $\begin{array}{l}a \\
a \\
a^{5} \mathrm{5}_{3} \\
a^{5} \mathrm{~F}_{4} \\
a^{5} \mathrm{~F}_{3} \\
a^{5} \mathrm{~F}_{2} \\
{ }^{5} \mathrm{~F}_{1}\end{array}$ & $\begin{array}{l}3162.80 \\
2968.22 \\
2808.76 \\
2687.01 \\
2604.82\end{array}$ & $\begin{array}{r}194.58 \\
159.46 \\
121.75 \\
82.19\end{array}$ & $\begin{array}{l}\text { 1. } 28: \\
1.30: \\
1.20 \\
0.97\end{array}$ & $\left\{\begin{array}{l}z^{5} \mathrm{P}^{\circ}, z^{5} \mathrm{D}^{\circ}, y{ }^{5} \mathrm{D}^{\circ}, x^{5} \mathrm{D}^{\circ}, z^{5} \mathrm{~F}^{\circ}, y^{5} \mathrm{~F}^{\circ}, z^{3} \mathrm{G}^{\circ} \\
y^{5} \mathrm{G}^{\circ} ?, x \mathrm{D}^{\circ}, z^{1} \mathrm{G}^{\circ}, z^{1} \mathrm{H}^{\circ}, z^{3} \mathrm{P}^{\circ}, z^{3} \mathrm{D}^{\circ}, z^{3} \mathrm{~F}^{\circ}, \\
y^{3} \mathrm{~F}^{\circ}, w^{3} \mathrm{~F}^{\circ}, u^{3} \mathrm{~F}^{\circ}, z^{3} \mathrm{G}^{\circ}, w^{3} \mathrm{G}^{\circ}, z^{3} \mathrm{I}^{\circ}, 1^{\circ} .\end{array}\right.$ \\
\hline $3 d^{3} 4 d\left({ }^{4} \mathrm{~F}\right)$ & $\begin{array}{l}e s \mathrm{P}_{3} \\
e \mathrm{~S}^{5} \mathrm{P}_{2} \\
e \mathrm{~S}^{\mathrm{S}} \mathrm{P}_{1}\end{array}$ & $\begin{array}{l}72908.17 \\
72674.28 \\
72517.84\end{array}$ & $\begin{array}{l}233.89 \\
156.44\end{array}$ & & $z^{5} \mathrm{D}^{\circ}, z^{5} \mathrm{G}^{\circ}, z^{3} \mathrm{D}^{\circ}, z^{3} \mathrm{~F}^{\circ}$. \\
\hline $3 d^{3} 4 d(4 \mathrm{~F})$ & $\begin{array}{l}e{ }^{5} \mathrm{D}_{4} \\
e{ }^{5} \mathrm{D}_{3} \\
e{ }^{5} \mathrm{D}_{2} \\
e{ }^{5} \mathrm{D}_{1} \\
e{ }^{5} \mathrm{D}_{0}\end{array}$ & $\begin{array}{l}\text { 72951. 00: } \\
72789.23: ? \\
72682.06: ?\end{array}$ & $\begin{array}{l}\text { 161. } 77 \\
107.17\end{array}$ & & $z^{5} \mathrm{D}^{\circ}, z^{5} \mathrm{~F}^{\circ}, z^{5} \mathrm{G}^{\circ}$. \\
\hline
\end{tabular}


TABLE 3.-Terms of the V II spectrum-Continued

\begin{tabular}{|c|c|c|c|c|c|}
\hline $\begin{array}{l}\text { Electron con- } \\
\text { figuration }\end{array}$ & $\begin{array}{l}\text { Term } \\
\text { symbol }\end{array}$ & Level & $\begin{array}{l}\text { Differ- } \\
\text { ence }\end{array}$ & $\begin{array}{c}\text { Ob- } \\
\text { served } \\
\sigma\end{array}$ & Combinations \\
\hline $3 d^{3} 4 d\left({ }^{4} \mathrm{~F}\right)$ & $\begin{array}{l}e e^{5} \mathrm{~F}_{5} \\
e e^{5} \mathrm{~F}_{4} \\
e^{3} \mathrm{~F}_{3} \\
e^{5} \mathrm{~F}_{2} \\
e^{5} \mathrm{~F}_{1}\end{array}$ & $\begin{array}{l}73293.82: ? \\
73222.72:\end{array}$ & 71.10 & & $z^{5} \mathrm{D}^{\circ}, z^{5} \mathrm{~F}^{\circ}, z^{5} \mathrm{G}^{\circ}$. \\
\hline $3 d^{3} 4 d\left({ }^{4} \mathrm{~F}\right)$ & $\begin{array}{l}e^{3} \mathrm{G}_{6} \\
e^{3} \mathrm{G}_{5} \\
e^{3} \mathrm{G}_{4} \\
e^{3} \mathrm{G}_{3} \\
e^{3} \mathrm{G}_{2}\end{array}$ & $\begin{array}{l}73498.93: \\
73416.63 \\
73278.92 \\
73145.68 \\
73026.76\end{array}$ & $\begin{array}{r}82.30 \\
137.71 \\
133.24 \\
118.92\end{array}$ & & $z^{3} \mathrm{D}^{\circ}, z^{5} \mathrm{~F}^{\circ}, z^{5} \mathrm{G}^{\circ}$ \\
\hline $3 d^{3} 4 d\left({ }^{4} \mathrm{~F}\right)$ & $\begin{array}{l}e s \mathrm{H}_{7} \\
e^{5} \mathrm{H}_{0} \\
e^{5} \mathrm{H}_{5} \\
e^{s} \mathrm{H}_{4} \\
e^{5} \mathrm{H}_{3}\end{array}$ & $\begin{array}{l}\text { 73020.35: } \\
72837.00: \\
72680.20: \\
72550.71 \\
72447.96:\end{array}$ & $\begin{array}{l}183.35 \\
156.80 \\
129.49 \\
102.75\end{array}$ & & $z^{5} \mathrm{G}^{\circ}, z^{3} \mathrm{G}^{\circ}$ \\
\hline $3 d^{3} 4 p\left({ }^{2} \mathrm{P}\right)$ & $z{ }^{1} \mathrm{~S}_{0}^{0}$ & 48258.28 & & & $a{ }^{1} \mathrm{P}, a^{3} \mathrm{P}, b{ }^{3} \mathrm{P}, c{ }^{3} \mathrm{P}, a^{3} \mathrm{D}, b^{3} \mathrm{D}$. \\
\hline $3 d^{3} 4 p\left({ }^{2} \mathrm{P}\right)$ & $z^{1} \mathrm{Pi}$ & 52803.75 & & 0.92 & $\left\{\begin{array}{c}a^{1 \mathrm{~S}}, a^{1} \mathrm{P}, a^{1} \mathrm{D}, b^{1} \mathrm{D}, a^{3} \mathrm{P}, b^{3} \mathrm{P}, c^{3} \mathrm{P}, a^{3} \mathrm{D}, b^{3} \mathrm{D}, \\
a^{3} \mathrm{~F}, b^{3} \mathrm{~F}, d^{3} \mathrm{~F}, a^{3} \mathrm{P} .\end{array}\right.$ \\
\hline $3 d^{3} 4 p\left({ }^{2} \mathrm{D}\right)$ & $y{ }^{1} \mathrm{P}_{1}^{0}$ & 56171.49 & & 1. 05: & $a{ }^{1} \mathrm{~S}, a^{1} \mathrm{P}, a^{1} \mathrm{D}, b^{1} \mathrm{D}, d^{3} \mathrm{P}, a^{3} \mathrm{D}$. \\
\hline $3 d^{3} 4 p\left({ }^{9} \mathrm{P}\right)$ & $z{ }^{1} \mathrm{D}_{2}$ & 49898.22 & & 0.93 & $\left\{\begin{array}{c}a^{1} \mathrm{P}, a^{1} \mathrm{D}, b{ }^{1} \mathrm{D}, b^{1} \mathrm{~F}, a^{3} \mathrm{P}, b^{3} \mathrm{P}, c^{3} \mathrm{P}, a^{3} \mathrm{D}, b^{3} \mathrm{D}, \\
\quad a^{3} \mathrm{~F}, b^{3} \mathrm{~F}, d^{3} \mathrm{~F}, a^{3} \mathrm{G}, b^{3} \mathrm{G} .\end{array}\right.$ \\
\hline $3 d^{3} 4 p\left({ }^{2} \mathrm{D}\right)$ & $y^{1} \mathrm{D}_{2}^{\mathbf{2}}$ & 57349.59 & & .98 & $a^{1} \mathrm{P}, b^{1} \mathrm{D}, a^{1} \mathrm{~F}, b^{1} \mathrm{~F}, b^{3} \mathrm{P}, d^{3} \mathrm{P}, a^{3} \mathrm{D}, b^{3} \mathrm{D}, c^{3} \mathrm{~F}$. \\
\hline $3 d^{3} 4 p\left({ }^{2} \mathrm{~F}\right)$ & $x{ }^{1} \mathrm{D}_{2}^{2}$ & 64586.29 & & 1. 03: & $\left\{\begin{array}{c}a^{1}{ }^{1} \mathrm{P}, a^{1} \mathrm{D}, b^{1} \mathrm{D}, c^{1} \mathrm{D}, a^{1} \mathrm{~F}, b^{1} \mathrm{~F}, c^{3} \mathrm{P}, a^{3} \mathrm{D}, b^{3} \mathrm{D}, \\
\quad a^{3} \mathrm{G}, a^{3} \mathrm{~F} .\end{array}\right.$ \\
\hline : & $w^{1} \mathrm{D}_{2}^{2}$ & 78791.3: & & & $a^{1} \mathrm{D}, b^{1} \mathrm{D}, a^{1} \mathrm{~F}, d^{3} \mathrm{~F}$. \\
\hline $3 d^{3} 4 p\left({ }^{2} \mathrm{G}\right)$ & $21 \mathrm{~F}_{3}^{\circ}$ & 49568.45 & & 0.97 & $\left\{\begin{array}{c}a^{1} \mathrm{D}, b^{1} \mathrm{D}, a^{1} \mathrm{~F}, b^{1} \mathrm{~F}, a^{1} \mathrm{G}, b^{1} \mathrm{G}, c^{3} \mathrm{P}, a^{3} \mathrm{D}, b^{3} \mathrm{D}, \\
a^{3} \mathrm{~F}, b^{3} \mathrm{~F}, d^{3} \mathrm{~F}, a^{3} \mathrm{G}, b^{3} \mathrm{G}, a^{3} \mathrm{H}, b^{3} \mathrm{H} .\end{array}\right.$ \\
\hline $3 d^{3} 4 p\left({ }^{2} \mathrm{D}\right)$ & $y^{1} \mathrm{~F}_{3}^{\circ}$ & 55142.01 & & .94 & $\left\{\begin{array}{l}a^{1} \mathrm{D}, b{ }^{1} \mathrm{D}, a^{1} \mathrm{~F}, a^{1} \mathrm{G}, b^{1} \mathrm{G}, c{ }^{1} \mathrm{G}, a^{3} \mathrm{P}, b^{3} \mathrm{P}, c^{3} \mathrm{P}, \\
a^{3} \mathrm{D}, b^{3} \mathrm{D}, a^{3} \mathrm{~F}, b^{3} \mathrm{~F}, c^{3} \mathrm{~F}, d^{3} \mathrm{~F}, a^{3} \mathrm{G}, b^{3} \mathrm{G}, a^{3} \mathrm{H}, \\
b^{3} \mathrm{H} .\end{array}\right.$ \\
\hline $3 d^{3} 4 p\left({ }^{2} \mathrm{~F}\right)$ & $x^{1} \mathrm{~F}_{3}^{\circ}$ & 66303.88 & & .95 & $a^{1} \mathrm{D}, b^{1} \mathrm{D}, c^{1} \mathrm{D}, b^{1} \mathrm{~F}, a^{1} \mathrm{G}, b^{1} \mathrm{G}, c^{1} \mathrm{G}, b^{3} \mathrm{~F}, b^{3} \mathrm{G}$. \\
\hline $3 d^{2} 4 s 4 p\left({ }^{2} \mathrm{G}\right)$ & $w^{1} \mathrm{~F}_{3}^{\circ}$ & 74664.5 & & & $a^{1} \mathrm{D}, a^{1} \mathrm{~F}, b^{1} \mathrm{G}, d^{3} \mathrm{~F}$. \\
\hline $3 d^{3} 4 p\left({ }^{2} \mathrm{G}\right)$ & $z^{1} \mathrm{G}_{4}$ & 49723.68 & & .96 & $\left\{\begin{array}{c}a^{1} \mathrm{G}, b^{1} \mathrm{G}, a^{1} \mathrm{H}, a^{3} \mathrm{D}, b^{3} \mathrm{D}, a^{3} \mathrm{~F}, b^{3} \mathrm{~F}, c^{3} \mathrm{~F}, a^{3} \mathrm{G}, \\
\quad b^{3} \mathrm{G}, a^{3} \mathrm{H}, b^{3} \mathrm{H}, a^{3} \mathrm{~F} .\end{array}\right.$ \\
\hline $3 d^{3} 4 p\left({ }^{2} \mathrm{H}\right)$ & $y^{1} \mathrm{G}_{4}^{\circ}$ & 54144.20 & & 1.00 & $\left\{\begin{array}{l}a^{1} \mathrm{~F}, b^{1} \mathrm{~F}, a^{1} \mathrm{G}, b^{1} \mathrm{G}, c^{1} \mathrm{G}, a^{1} \mathrm{H}, a^{3} \mathrm{G}, b^{3} \mathrm{G}, a^{3} \mathrm{H}, \\
b^{3} \mathrm{H} .\end{array}\right.$ \\
\hline $3 d^{3} 4 p\left({ }^{2} \mathrm{~F}\right)$ & $x^{1} G_{i}$ & 65790.28 & & 0.94 & $a^{1} \mathrm{~F}, b^{1} \mathrm{~F}, a^{1} \mathrm{G}, b^{1} \mathrm{G}, c^{1} \mathrm{G}, a^{1} \mathrm{H}, a^{3} \mathrm{D}, d^{3} \mathrm{~F}$. \\
\hline $3 d^{2} 4 s 4 p\left({ }^{2} \mathrm{G}\right)$ & $w^{1} \mathrm{G}_{i}$ & 72292.2: & & & $b^{1} \mathrm{~F}, a^{1} \mathrm{G}, b^{1} \mathrm{G}, a^{1} \mathrm{H}, b^{3} \mathrm{H}$. \\
\hline $3 d^{3} 4 p\left({ }^{2} \mathrm{G}\right)$ & $2{ }^{1} \mathrm{H}_{5}^{\circ}$ & 49593.41 & & .95 & $\left\{\begin{array}{l}a^{1} \mathrm{G}, b^{1} \mathrm{G}, a^{1} \mathrm{H}, a^{1} \mathrm{I}, a^{3} \mathrm{~F}, b^{3} \mathrm{~F}, a^{3} \mathrm{G}, b^{3} \mathrm{G}, a^{3} \mathrm{H}, \\
\quad b^{3} \mathrm{H}, a^{6} \mathrm{~F} .\end{array}\right.$ \\
\hline $3 d^{3} 4 p\left({ }^{2} \mathrm{H}\right)$ & $y^{1} \mathrm{H}_{5}^{\circ}$ & 55499.38 & & 1. 03: & $\left\{\begin{array}{c}a^{1} \mathrm{G}, b^{1} \mathrm{G}, c^{1} \mathrm{G}, a^{1} \mathrm{H}, a^{1} \mathrm{I}, a^{3} \mathrm{~F}, b^{3} \mathrm{~F}, c^{3} \mathrm{~F}, d^{3} \mathrm{~F}, \\
a^{3} \mathrm{G}, b^{3} \mathrm{G}, a^{3} \mathrm{H}, b^{3} \mathrm{H} .\end{array}\right.$ \\
\hline $3 d^{2} 4 s 4 p\left({ }^{2} \mathrm{G}\right)$ & $x{ }^{1} \mathrm{H}_{5}^{\circ}$ & 70936.4 & & & $a^{1} \mathrm{G}, b^{1} \mathrm{G}, a^{1} \mathrm{I}, b^{3} \mathrm{H}$. \\
\hline $3 d^{3} 4 p\left({ }^{2} \mathrm{H}\right)$ & $21^{1}{ }_{6}^{\circ}$ & 55403.38 & & 1. 01: & $a^{1} \mathrm{H}, a^{1} \mathrm{I}, a^{3} \mathrm{H}, b^{3} \mathrm{H}$. \\
\hline $3 d^{3} 4 p\left({ }^{2} \mathrm{P}\right)$ & $z{ }^{3} \mathrm{Si}$ & 52181.18 & & 1.85 & $a^{3} \mathrm{P}, b^{3} \mathrm{P}, c^{3} \mathrm{P}, d^{3} \mathrm{P}, a^{5} \mathrm{D} ?$ \\
\hline $3 d^{2} 4 p\left({ }^{4} \mathrm{P}\right)$ & $y{ }^{3} \mathrm{Si}$ & 55663.27 & & 1.92 & $a^{3} \mathrm{P}, c^{3} \mathrm{P}, d^{3} \mathrm{P}, b^{3} \mathrm{D}, a^{1} \mathrm{P}, a^{3} \mathrm{P}, a^{5} \mathrm{D}$ \\
\hline $3 d^{3} 4 p\left({ }^{4} \mathrm{P}\right)$ & $\begin{array}{l}z^{3} \mathrm{P}_{2}^{\circ} \\
z^{3} \mathrm{P}_{1}^{\circ} \\
z^{3} \mathrm{P}_{0}^{0}\end{array}$ & $\begin{array}{l}46799.98 \\
46690.43 \\
46586.43\end{array}$ & $\begin{array}{r}49.55 \\
104.00\end{array}$ & $\begin{array}{l}1.48 \\
1.44\end{array}$ & $\left\{\begin{array}{l}a^{3} \mathrm{P}, b^{3} \mathrm{P}, c^{3} \mathrm{P}, d^{3} \mathrm{P}, a^{3} \mathrm{D}, b^{3} \mathrm{D}, a^{3} \mathrm{~F}, a^{1} \mathrm{~S}, \\
a^{1} \mathrm{D}, a^{3} \mathrm{P}, a^{3} \mathrm{D}, a^{3} \mathrm{~F} .\end{array}\right.$ \\
\hline $3 d^{3} 4 p\left({ }^{2} \mathrm{P}\right)$ & $\begin{array}{l}y{ }^{3} \mathrm{P}_{2}^{\circ} \\
y{ }^{3} \mathrm{P}^{0} \\
y{ }^{3} \mathrm{P}_{0}^{0}\end{array}$ & $\begin{array}{l}51123.31 \\
50738.82 \\
50662.36\end{array}$ & $\begin{array}{r}384.49 \\
76.46\end{array}$ & $\begin{array}{l}1.51 \\
1.39\end{array}$ & $\left\{\begin{array}{l}a^{3} \mathrm{P}, b^{3} \mathrm{P}, c^{3} \mathrm{P}, a^{3} \mathrm{D}, b^{3} \mathrm{D}, a^{3} \mathrm{~F}, a^{1} \mathrm{P}, a^{1} \mathrm{D}, \\
a^{3} \mathrm{P} .\end{array}\right.$ \\
\hline $3 d^{3} 4 p\left({ }^{2} \mathrm{D}\right)$ & $\begin{array}{l}x^{3} \mathrm{P}_{2}^{0} \\
x^{3} \mathrm{P}_{1}^{1} \\
x^{3} \mathrm{P}_{0}^{0}\end{array}$ & $\begin{array}{l}54715.69 \\
54717.85 \\
54818.45\end{array}$ & $\begin{array}{r}-2.22 \\
-95.60\end{array}$ & & $\left\{\begin{array}{l}a^{3} \mathrm{P}, b^{3} \mathrm{P}, c^{3} \mathrm{P}, d^{3} \mathrm{P}, a^{3} \mathrm{D}, b^{3} \mathrm{D}, a^{1} \mathrm{~S}, a^{1} \mathrm{P} . \\
a^{1} \mathrm{D}, b^{1} \mathrm{D} .\end{array}\right.$ \\
\hline
\end{tabular}


TABLE 3.-Terms of the V II spectrum-Continued

\begin{tabular}{|c|c|c|c|c|c|}
\hline $\begin{array}{l}\text { Electron con- } \\
\text { figuration }\end{array}$ & $\begin{array}{c}\text { Term } \\
\text { symbol }\end{array}$ & Level & $\begin{array}{l}\text { Differ- } \\
\text { ence }\end{array}$ & $\begin{array}{c}\text { Ob- } \\
\text { served } \\
g\end{array}$ & Combinations \\
\hline $3 d^{3} 4 p\left({ }^{4} \mathrm{~F}\right)$ & $\begin{array}{l}z^{3} \mathrm{D}_{3}^{\circ} \\
z^{3} \mathrm{D}_{2}^{2} \\
2{ }^{3} \mathrm{D}_{\mathrm{i}}\end{array}$ & $\begin{array}{l}\$ 7205.01 \\
\$ 7041.11 \\
\$ 6954.58\end{array}$ & $\begin{array}{r}163.90 \\
86.53\end{array}$ & $\begin{array}{l}1.32 \\
1.08 \\
0.24\end{array}$ & $\left\{\begin{array}{l}a^{3} \mathrm{P}, c^{3} \mathrm{P}, a^{3} \mathrm{D}, b^{3} \mathrm{D}, a^{3} \mathrm{~F}, b^{3} \mathrm{~F}, b^{3} \mathrm{G}, a^{1} \mathrm{~S}, \\
a^{3} \mathrm{P}, e^{5} \mathrm{P}, a^{3} \mathrm{D}, a^{3} \mathrm{~F} .\end{array}\right.$ \\
\hline $3 d^{3} 4 p\left({ }^{4} \mathrm{P}\right)$ & $\begin{array}{l}y^{3}{ }^{3} \mathrm{D}_{3}^{\circ} \\
y^{3} \mathrm{D}_{2}^{\circ} \\
\left.y{ }^{3} \mathrm{~T}\right)_{1}^{\circ}\end{array}$ & $\begin{array}{l}51085.77 \\
50775.47 \\
50473.76\end{array}$ & $\begin{array}{l}310.30 \\
301.71\end{array}$ & $\begin{array}{l}1.27 \\
1.11 \\
0.49\end{array}$ & $\left\{\begin{array}{l}a^{3} \mathrm{P}, b^{3} \mathrm{P}, c^{3} \mathrm{P}, d^{3} \mathrm{P}, a^{3} \mathrm{D}, b^{3} \mathrm{D}, a^{3} \mathrm{~F}, b^{3} \mathrm{~F}, \\
c^{3} \mathrm{~F}, d^{3} \mathrm{~F}, a^{3} \mathrm{G}, a^{1} \mathrm{~S}, a^{1} \mathrm{P}, a^{1} \mathrm{D}, b^{1} \mathrm{D}, a^{3} \mathrm{P} .\end{array}\right.$ \\
\hline $3^{d^{3}} 4 p\left({ }^{2} \mathrm{P}\right)$ & $\begin{array}{l}x^{3} \mathrm{D}_{3}^{2} \\
x^{3} \mathrm{D}_{2}^{\circ} \\
x^{3} \mathrm{D}_{1}^{0}\end{array}$ & $\begin{array}{l}52767.36 \\
52700.03 \\
52604.11\end{array}$ & $\begin{array}{l}67.33 \\
95.92\end{array}$ & $\begin{array}{l}1.26 \\
1.10 \\
0.63\end{array}$ & $\left\{\begin{array}{l}a^{3} \mathrm{P}, b^{3} \mathrm{P}, c^{3} \mathrm{P}, d^{3} \mathrm{P}, a^{3} \mathrm{D}, b^{3} \mathrm{D}, a^{3} \mathrm{~F}, b^{3} \mathrm{~F}, \\
c^{3} \mathrm{~F}, d^{3} \mathrm{~F}, a^{3} \mathrm{G}, a^{1} \mathrm{~S}, a^{1} \mathrm{P}, a^{1} \mathrm{D}, b^{1} \mathrm{D}, a^{3} \mathrm{P} .\end{array}\right.$ \\
\hline $3 d^{3} 4 p\left({ }^{2} \mathrm{D}\right)$ & $\begin{array}{l}w^{3} \mathrm{D}_{3}^{\circ} \\
w^{3} \mathrm{D}_{2}^{\circ} \\
w^{3} \mathrm{D}_{1}^{\circ}\end{array}$ & $\begin{array}{l}58927.19 \\
53868.63 \\
53751.46\end{array}$ & $\begin{array}{r}58.56 \\
117.17\end{array}$ & $\begin{array}{l}1.37 \\
1.10 \\
0.49:\end{array}$ & $\left\{\begin{array}{l}a^{3} \mathrm{P}, b^{3} \mathrm{P}, c^{3} \mathrm{P}, a^{3} \mathrm{D}, b^{3} \mathrm{D}, a^{3} \mathrm{~F}, b^{3} \mathrm{~F}, c^{3} \mathrm{~F}, \\
d^{3} \mathrm{~F}, a^{3} \mathrm{G}, a^{1} \mathrm{~S}, a^{1} \mathrm{D}, b^{1} \mathrm{D}, a^{1} \mathrm{~F}, b^{1} \mathrm{G} .\end{array}\right.$ \\
\hline $3 d^{3} 4 p\left({ }^{2} F\right)$ & $\begin{array}{l}v{ }^{3} \mathrm{D}_{3}^{2} \\
v{ }^{3} \mathrm{D}_{2}^{2} \\
v{ }^{3} \mathrm{D}_{1}^{\circ}\end{array}$ & $\begin{array}{l}64603.53 \\
64804.18 \\
64930.76\end{array}$ & $\begin{array}{l}-200.60 \\
-126.63\end{array}$ & $\begin{array}{l}\text { 1. } 22: \\
\text { 1. } 02: \\
0.46:\end{array}$ & $\left\{\begin{array}{l}a^{3} \mathrm{P}, b^{3} \mathrm{P}, c^{3} \mathrm{P}, d^{3} \mathrm{P}, a^{3} \mathrm{D}, b^{3} \mathrm{D}, c^{3} \mathrm{D}, a^{3} \mathrm{~F}, \\
b^{3} \mathrm{~F}, c^{3} \mathrm{~F}, d^{3} \mathrm{~F}, a^{1} \mathrm{~F}, a^{5} \mathrm{D} .\end{array}\right.$ \\
\hline $3 d^{24} 44 p(4 \mathrm{~F})$ & $\begin{array}{l}u^{3} \mathrm{D}_{3}^{\circ} \\
u^{3} \mathrm{D}_{2}^{8} \\
u^{3} \mathrm{D}_{1}^{\circ}\end{array}$ & $\begin{array}{l}68945.0 \\
68797.7 \\
68759.4\end{array}$ & $\begin{array}{r}147.3 \\
38.3\end{array}$ & & $\left\{\begin{array}{l}a^{3} \mathrm{P}, b^{3} \mathrm{P}, c^{3} \mathrm{P}, d^{3} \mathrm{P}, a^{3} \mathrm{D}, b^{3} \mathrm{D}, a^{3} \mathrm{~F}, b^{3} \mathrm{~F}, \\
c^{3} \mathrm{~F}, b^{3} \mathrm{G} ?, a^{1 \mathrm{~S}}, a^{1} \mathrm{~F}, b^{1} \mathrm{G}, a^{5} \mathrm{P} .\end{array}\right.$ \\
\hline $3 d^{3} 4 p\left(\right.$ high $\left.^{2} \mathrm{D}\right) ?$ & $\begin{array}{l}t{ }^{3} \mathrm{D}_{3}^{\circ} \\
t \mathrm{D}_{2}^{\circ} \\
t{ }^{3} \mathrm{D}_{1}^{\circ}\end{array}$ & $\begin{array}{l}75848.19 \\
75758.29 \\
75715.45:\end{array}$ & $\begin{array}{l}89.84 \\
42.84\end{array}$ & $\begin{array}{l}(1.27:) \\
(1.14:) \\
(0.50:)\end{array}$ & $b^{3} \mathrm{P}, c^{3} \mathrm{P}, a^{3} \mathrm{D} ?, b^{3} \mathrm{D}, c^{3} \mathrm{D}, c^{3} \mathrm{~F}, d^{3} \mathrm{~F}$ \\
\hline $3 d^{3} 4 p\left({ }^{4} \mathrm{~F}\right)$ & $\begin{array}{l}z 3 \mathrm{~F}_{4}^{\circ} \\
z 3 \mathrm{~F}_{3}^{0} \\
z 3 \mathrm{~F}_{2}^{0}\end{array}$ & $\begin{array}{l}40480.10 \\
40195.52 \\
40001.66\end{array}$ & $\begin{array}{l}234.58 \\
193.86\end{array}$ & $\begin{array}{l}1.22 \\
1.02 \\
0.65\end{array}$ & $\left\{\begin{array}{l}a^{3} \mathrm{P}, a^{3} \mathrm{D}, b^{3} \mathrm{D}, a^{3} \mathrm{~F}, b^{3} \mathrm{~F}, a^{3} \mathrm{G}, b^{3} \mathrm{G}, a^{1} \mathrm{D}, \\
a^{1} \mathrm{G}, e^{5} \mathrm{P}, a^{5} \mathrm{D}, a^{5} \mathrm{~F} .\end{array}\right.$ \\
\hline $3 d^{3} 4 p\left({ }^{2} \mathrm{G}\right)$ & $\begin{array}{l}y{ }^{3} \mathrm{~F}_{4}^{0} \\
y^{3} \mathrm{~F}_{3}^{0} \\
y^{3} \mathrm{~F}_{2}^{0}\end{array}$ & $\begin{array}{l}49268.61 \\
49210.78 \\
49201.66\end{array}$ & $\begin{array}{r}57.83 \\
9.12\end{array}$ & $\begin{array}{r}1.18 \\
0.99 \\
.63\end{array}$ & $\left\{\begin{array}{l}b^{3} \mathrm{P}, c^{3} \mathrm{P}, a^{3} \mathrm{D}, b^{3} \mathrm{D}, a^{3} \mathrm{~F}, b^{3} \mathrm{~F}, c^{3} \mathrm{~F}, a^{3} \mathrm{G}, \\
b^{3} \mathrm{G}, a^{3} \mathrm{H}, b^{3} \mathrm{H}, a^{1} \mathrm{P}, a^{1} \mathrm{D}, b^{1} \mathrm{D}, b^{1} \mathrm{~F}, a^{1} \mathrm{G}, \\
b^{1} \mathrm{G}, a^{3} \mathrm{~F} .\end{array}\right.$ \\
\hline $3 d^{3} 4 p\left({ }^{2} \mathrm{D}\right)$ & $\begin{array}{l}x^{3} \mathrm{~F}_{4}^{\circ} \\
x 3 \mathrm{~F}_{3}^{0} \\
x 3 \mathrm{~F}_{2}^{0}\end{array}$ & $\begin{array}{l}52657.51 \\
52391.94 \\
52245.68\end{array}$ & $\begin{array}{l}265.57 \\
146.26\end{array}$ & $\begin{array}{l}\text { 1. } 18: \\
\text { 1. } 07 \\
0.68\end{array}$ & $\left\{\begin{array}{l}a^{3 \mathrm{P}}, b^{3} \mathrm{P}, c^{3} \mathrm{P}, d^{3 \mathrm{P}}, a^{3} \mathrm{D}, b^{3 \mathrm{D}}, a^{3} \mathrm{~F}, b^{3} \mathrm{~F}, \\
c^{3} \mathrm{~F}, d^{3} \mathrm{~F}, a^{3} \mathrm{G}, a^{3} \mathrm{H}, a^{1} \mathrm{P}, a^{1} \mathrm{D}, b^{1} \mathrm{D}, a^{1} \mathrm{H} .\end{array}\right.$ \\
\hline $3 d^{3} 4 p\left({ }^{2} \mathrm{~F}\right)$ & $\begin{array}{l}w^{3} \mathrm{~F}_{4}^{\circ} \\
w^{3} \mathrm{~F}_{3}^{\circ} \\
w^{3} \mathrm{~F}_{2}^{\circ}\end{array}$ & $\begin{array}{l}62176.24 \\
62133.39 \\
62085.02\end{array}$ & $\begin{array}{l}42.85 \\
48.37\end{array}$ & $\begin{array}{l}\text { 1. } 36: \\
1.00 \\
0.58:\end{array}$ & $\left\{\begin{array}{l}c^{3} \mathrm{P}, a^{3} \mathrm{D}, b^{3} \mathrm{D}, c^{3} \mathrm{D}, a^{3} \mathrm{~F}, b^{3} \mathrm{~F}, c^{3} \mathrm{~F}, d^{3} \mathrm{~F}, \\
a^{3} \mathrm{G}, b^{3} \mathrm{G}, a^{3} \mathrm{H}, a^{1} \mathrm{H}, a^{5} \mathrm{~F} .\end{array}\right.$ \\
\hline $3 d^{2} 4 s 4 p\left({ }^{4} \mathrm{~F}\right)$ & $\begin{array}{l}v^{3} \mathrm{~F}_{4}^{0} \\
v^{3} \mathrm{~F}_{3}^{3} \\
v{ }^{3} \mathrm{~F}_{2}^{0}\end{array}$ & $\begin{array}{l}68147.2 \\
67905.1 \\
67787.8\end{array}$ & $\begin{array}{l}242.1 \\
167.3\end{array}$ & & $\left\{\begin{array}{l}b^{3} \mathrm{P} ?, c^{3} \mathrm{P}, a^{3} \mathrm{D}, b^{3} \mathrm{D}, a^{3} \mathrm{~F}, b^{3} \mathrm{~F}, c^{3} \mathrm{~F}, d^{3} \mathrm{~F}, \\
a^{3} \mathrm{G}, b^{3} \mathrm{G}, a^{1} \mathrm{D} .\end{array}\right.$ \\
\hline & $\begin{array}{l}u^{3} \mathrm{~F}_{4}^{0} \\
u^{3} \mathrm{~F}_{3}^{0} \\
u^{3} \mathrm{~F}_{2}^{0}\end{array}$ & $\begin{array}{l}76649.5 \\
76385.8 \\
76220.4\end{array}$ & $\begin{array}{l}257.7 \\
165.4\end{array}$ & & $\left\{\begin{array}{c}c^{3} \mathrm{P} ?, a^{3} \mathrm{D}, b^{3} \mathrm{D}, c^{3} \mathrm{D}, c^{3} \mathrm{~F}, d^{3} \mathrm{~F}, a^{3} \mathrm{G}, b^{3} \mathrm{G}, \\
b^{3} \mathrm{H}, a^{1} \mathrm{D}, a^{1} \mathrm{H}, a^{5} \mathrm{D}, a^{5} \mathrm{~F} .\end{array}\right.$ \\
\hline $3 d^{3} 4 p(4 \mathrm{~F})$ & $\begin{array}{l}2 \mathrm{z}^{3} \mathrm{G}_{5}^{\circ} \\
z^{3} \mathrm{G}_{4}^{\circ} \\
2^{3} \mathrm{G}_{3}^{\circ}\end{array}$ & $\begin{array}{l}39612.97 \\
39403.77 \\
\text { 39294.05 }\end{array}$ & $\begin{array}{l}209.20 \\
169.72\end{array}$ & $\begin{array}{l}1.19 \\
1.03 \\
0.84\end{array}$ & $\left\{\begin{array}{l}a^{3} \mathrm{~F}, b^{3} \mathrm{~F}, a^{3} \mathrm{G}, b^{3} \mathrm{G}, a^{3} \mathrm{H}, b^{3} \mathrm{H}, b^{1} \mathrm{G}, a^{5} \mathrm{D}, a^{5} \mathrm{~F}, \\
e^{5} \mathrm{H} .\end{array}\right.$ \\
\hline $3 d^{3} 4 p\left({ }^{2} \mathrm{G}\right)$ & $\begin{array}{l}y^{3} \mathrm{G}_{5}^{\circ} \\
y^{3} \mathrm{G}_{4}^{\circ} \\
y^{3} \mathrm{G}_{3}^{\circ}\end{array}$ & $\begin{array}{l}48858.04 \\
48730.76 \\
48579.96\end{array}$ & $\begin{array}{l}122.28 \\
150.80\end{array}$ & $\begin{array}{l}1.22 \\
1.02 \\
0.67\end{array}$ & $\left\{\begin{array}{c}b^{3} \mathrm{P}, a^{3} \mathrm{D}, a^{3} \mathrm{~F}, b^{3} \mathrm{~F}, a^{3} \mathrm{G}, b^{3} \mathrm{G}, a^{3} \mathrm{H}, b^{3} \mathrm{H}, \\
a^{1} \mathrm{D}, a^{1} \mathrm{G}, b^{1} \mathrm{G}, a^{1} \mathrm{H}, a^{1} \mathrm{~L} .\end{array}\right.$ \\
\hline $3 d^{3} 4 p\left({ }^{2} \mathrm{H}\right)$ & $\begin{array}{l}x^{3} \mathrm{G}: \\
x^{3} \mathrm{G}_{4}^{0} \\
x^{3} \mathrm{G}_{3}^{6}\end{array}$ & $\begin{array}{l}55206.87 \\
55304.34 \\
55349.63\end{array}$ & $\begin{array}{l}-97.47 \\
-45.29\end{array}$ & $\begin{array}{l}1.15 \\
1.02 \\
0.82\end{array}$ & $\left\{\begin{array}{c}b^{3} \mathrm{D}, a^{3} \mathrm{~F}, b^{3} \mathrm{~F}, c^{3} \mathrm{~F}, d^{3} \mathrm{~F}, a^{3} \mathrm{G}, b^{3} \mathrm{G}, a^{3} \mathrm{H}, \\
b^{3} \mathrm{H}, a^{1} \mathrm{~F}, a^{1} \mathrm{G}, b^{1} \mathrm{G}, c^{1} \mathrm{G}, a^{1} \mathrm{H}, a^{1} \mathrm{I} .\end{array}\right.$ \\
\hline $3 d^{3} 4 p\left({ }^{2} \mathrm{~F}\right)$ & $\begin{array}{l}w^{3} \mathrm{G}_{5}^{\circ} \\
w^{3} \mathrm{G}_{4}^{\circ} \\
w^{3} \mathrm{G}_{3}^{\circ}\end{array}$ & $\begin{array}{l}64229.10 \\
64130.84 \\
64057.89\end{array}$ & $\begin{array}{l}98.26 \\
73.45\end{array}$ & $\begin{array}{l}\text { 1. } 02 \\
0.72:\end{array}$ & $\left\{\begin{array}{l}a^{3} \mathrm{~F}, b^{3} \mathrm{~F}, c^{3} \mathrm{~F}, d^{3} \mathrm{~F}, a^{3} \mathrm{G}, b^{3} \mathrm{G}, a^{3} \mathrm{H}, b^{3} \mathrm{H}, a^{1} \mathrm{~F}, \\
b^{1} \mathrm{~F}, c^{1} \mathrm{G}, a^{1} \mathrm{H}, a^{3} \mathrm{~F} .\end{array}\right.$ \\
\hline $3 d^{2} 484 p\left({ }^{4} \mathrm{~F}\right)$ & $\begin{array}{l}v^{3} \mathrm{G}_{5}^{0} \\
v^{3} \mathrm{G}_{4}^{\circ} \\
v^{3} \mathrm{G}_{3}^{0}\end{array}$ & $\begin{array}{l}70227.8 \\
69912.1 \\
69644.2\end{array}$ & $\begin{array}{l}315.7 \\
267.9\end{array}$ & & $b^{3} \mathrm{~F}, c^{3} \mathrm{~F}, d^{3} \mathrm{~F}, a^{3} \mathrm{G}, b^{3} \mathrm{G}, b^{3} \mathrm{H}, a^{1} \mathrm{D}, a^{1} \mathrm{G}, b^{1} \mathrm{G}$. \\
\hline $3 d^{3} 4 p\left({ }^{2} \mathrm{G}\right)$ & $\begin{array}{l}z^{3} \mathrm{H}_{6}^{\circ} \\
z^{3} \mathrm{H}_{5}^{0} \\
z^{3} \mathrm{H}_{4}^{\circ}\end{array}$ & $\begin{array}{l}47607.79 \\
47297.08 \\
47056.32\end{array}$ & $\begin{array}{l}310.71 \\
240.76\end{array}$ & $\begin{array}{l}1.13 \\
1.01 \\
0.78\end{array}$ & $b^{3} \mathrm{~F}, d^{3} \mathrm{~F}, a^{3} \mathrm{G}, b^{3} \mathrm{G}, a^{3} \mathrm{H}, b^{3} \mathrm{H}, a^{1} \mathrm{G}, b^{1} \mathrm{G}$. \\
\hline $3 d^{3} 4 p\left({ }^{2} \mathrm{H}\right)$ & $\begin{array}{l}y^{3} \mathrm{H}_{6}^{\circ} \\
y^{3} \mathrm{H}_{5}^{\circ} \\
y^{3} \mathrm{H}_{i}^{\circ}\end{array}$ & $\begin{array}{l}52252.70 \\
52153.55 \\
52082.88\end{array}$ & $\begin{array}{l}99.15 \\
70.67\end{array}$ & $\begin{array}{l}1.04: \\
0.98 \\
.70\end{array}$ & $\begin{array}{l}a^{3} F, b^{3} F, a^{3} \mathrm{G}, b^{3} \mathrm{G}, a^{3} \mathrm{H}, b^{3} \mathrm{H}, a^{1} \mathrm{~F}, a^{1} \mathrm{G}, b^{1} \mathrm{G}, \\
a^{1} \mathrm{H} .\end{array}$ \\
\hline
\end{tabular}


TABLE 3.-Terms of the V II spectrum-Continued

\begin{tabular}{|c|c|c|c|c|c|}
\hline $\begin{array}{l}\text { Electron con- } \\
\text { figuration }\end{array}$ & $\begin{array}{c}\text { Term } \\
\text { symbol }\end{array}$ & Level & $\begin{array}{l}\text { Differ- } \\
\text { ence }\end{array}$ & $\begin{array}{l}\text { Ob- } \\
\text { served } \\
g\end{array}$ & Combinations \\
\hline $3 d^{3} 4 \mathrm{p}\left({ }^{2} \mathrm{H}\right)$ & $\begin{array}{l}z^{3} \mathrm{I}_{7}^{\circ} \\
z^{3} \mathrm{I}_{6}^{\circ} \\
z^{3} \mathrm{I}_{5}^{\circ}\end{array}$ & $\begin{array}{l}53319.52 \\
53076.82 \\
52877.99\end{array}$ & $\begin{array}{l}242.70 \\
198.83\end{array}$ & $\begin{array}{l}1.11: \\
0.98 \\
.84:\end{array}$ & $a^{3} \mathrm{H}, b^{3} \mathrm{H}, b^{1} \mathrm{G}, a^{1} \mathrm{H}, a^{5} \mathrm{~F}$. \\
\hline $3 d^{3} 4 p\left({ }^{4} \mathrm{P}\right)$ & $z^{5} \mathrm{~S}_{2}^{2}$ & 49751.32 & & & $a^{5} \mathrm{P}, a^{3} \mathrm{P}, c^{3} \mathrm{P}, a^{3} \mathrm{D}, b^{3} \mathrm{D}, b^{3} \mathrm{~F}$. \\
\hline $3 d^{3} 4 p\left({ }^{4} \mathrm{P}\right)$ & $\begin{array}{l}z^{5} \mathrm{P}_{3}^{\circ} \\
z^{5} \mathrm{P}_{2}^{\circ} \\
z^{3} \mathrm{P}_{1}^{\circ}\end{array}$ & $\begin{array}{l}47051.89 \\
46879.94 \\
46754.59\end{array}$ & $\begin{array}{l}\text { 171. } 95 \\
125.35\end{array}$ & $\begin{array}{l}\text { 1. } 55 \\
\text { 1. } 68 \\
2.28\end{array}$ & $a^{5} \mathrm{P}, a^{5} \mathrm{D}, a^{5} \mathrm{~F}, b^{3} \mathrm{P}, c^{3} \mathrm{P}$. \\
\hline $3 d^{3} 4 p\left({ }^{4} \mathrm{~F}\right)$ & $\begin{array}{l}z^{5} \mathrm{D}_{i}^{0} \\
z^{5} \mathrm{D}_{3}^{0} \\
z^{5} \mathrm{D}_{2}^{0} \\
z^{5} \mathrm{D}_{1}^{0} \\
z^{5} \mathrm{D}_{0}^{0}\end{array}$ & $\begin{array}{l}37531.09 \\
37520.61 \\
37369.01 \\
37259.42 \\
\text { 37201. } 41\end{array}$ & $\begin{array}{r}10.48 \\
151.60 \\
109.59 \\
58.01\end{array}$ & $\begin{array}{l}1.44 \\
1.47 \\
1.39 \\
1.39\end{array}$ & $\left\{\begin{array}{l}a^{5} \mathrm{P}, e^{5} \mathrm{P}, a^{5} \mathrm{D}, e^{5} \mathrm{D}, a^{5} \mathrm{~F}, e^{5} \mathrm{~F}, e^{5} \mathrm{G}, a^{3} \mathrm{P}, c^{3} \mathrm{P}, \\
a^{3} \mathrm{D}, b^{3} \mathrm{D}, a^{3} \mathrm{~F}, b^{3} \mathrm{~F}, a^{3} \mathrm{G} .\end{array}\right.$ \\
\hline $3 d^{3} 4 p\left({ }^{4} \mathrm{P}\right)$ & $\begin{array}{l}y{ }^{5} \mathrm{D}_{4}^{0} \\
y^{5} \mathrm{D}_{3}^{5} \\
y^{5} \mathrm{D}_{2}^{5} \\
y^{5} \mathrm{D}_{1}^{0} \\
y^{5} \mathrm{D}_{0}^{0}\end{array}$ & $\begin{array}{l}47420.10 \\
47181.17 \\
47101.88 \\
47107.98 \\
47027.88\end{array}$ & $\begin{array}{r}238.93 \\
79.29 \\
-6.10 \\
80.10\end{array}$ & $\begin{array}{l}2.28 \\
1.48: \\
1.47 \\
1.43\end{array}$ & $\left\{\begin{array}{l}a^{5} \mathrm{P}, a^{5} \mathrm{D}, a^{5} \mathrm{~F}, a^{1} \mathrm{~S}, a^{1} \mathrm{P}, a^{3} \mathrm{P}, b^{3} \mathrm{P}, c^{3} \mathrm{P}, a^{3} \mathrm{D}, \\
b^{3} \mathrm{D}, a^{3} \mathrm{~F} .\end{array}\right.$ \\
\hline $3 d^{2} 484 p\left({ }^{4} \mathrm{~F}\right)$ & $\begin{array}{l}x^{5} \mathrm{D}_{4}^{\circ} \\
x^{5} \mathrm{D}_{3}^{0} \\
x^{5} \mathrm{D}_{2}^{\circ} \\
x^{5} \mathrm{D}_{1}^{8} \\
x^{5} \mathrm{D}_{0}^{\circ}\end{array}$ & $\begin{array}{l}66158.6 \\
65996.7 \\
65885.3 \\
65816.2 \\
65783.4\end{array}$ & $\begin{array}{r}161.9 \\
111.4 \\
69.1 \\
32.8\end{array}$ & & $a^{5} \mathrm{P}, a^{5} \mathrm{~F}, a^{3} \mathrm{P}, a^{3} \mathrm{D}, a^{3} \mathrm{~F}, b^{3} \mathrm{~F}, c^{3} \mathrm{~F}, a^{3} \mathrm{G}$. \\
\hline $3 d^{3} 4 p\left({ }^{4} \mathrm{~F}\right)$ & $\begin{array}{l}z^{5}{ }^{5} \mathrm{~F}_{5}^{\circ} \\
z^{5} \mathrm{~F}_{4}^{\circ} \\
z^{6} \mathrm{~F}_{3}^{\circ} \\
z^{5} \mathrm{~F}_{2}^{\circ} \\
z^{5} \mathrm{~F}_{1}^{\circ}\end{array}$ & $\begin{array}{l}37352.39 \\
37150.57 \\
36919.23 \\
36673.51 \\
36489.34\end{array}$ & $\begin{array}{l}201.82 \\
231.34 \\
245.72 \\
184.17\end{array}$ & $\begin{array}{l}1.40: \\
1.24 \\
1.08 \\
0.35\end{array}$ & $\left\{\begin{array}{l}a^{5} \mathrm{P}, a^{5} \mathrm{D}, e^{5} \mathrm{D}, a^{5} \mathrm{~F}, e^{5} \mathrm{~F}, e^{5} \mathrm{G}, a^{1} \mathrm{~S}, a^{3} \mathrm{P}, c^{3} \mathrm{P} \\
a^{3} \mathrm{D}, b^{3} \mathrm{D}, a^{3} \mathrm{~F}, b^{3} \mathrm{~F}, a^{3} \mathrm{G}, a^{3} \mathrm{H} .\end{array}\right.$ \\
\hline $3 d^{24 s 4 p(4 \mathrm{~F})}$ & 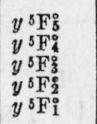 & $\begin{array}{l}64287.1 \\
64026.6 \\
63816.9 \\
63657.2 \\
63548.5\end{array}$ & $\begin{array}{l}260.5 \\
209.7 \\
159.7 \\
108.7\end{array}$ & & $a^{5} \mathrm{~F}, a^{1} \mathrm{G}, a^{3} \mathrm{G}, a^{3} \mathrm{H}$. \\
\hline $3 d^{3} 4 p\left({ }^{4} \mathrm{~F}\right)$ & $\begin{array}{l}z^{5} \mathrm{G}_{6}^{\circ} \\
z^{5} \mathrm{G}_{5}^{\circ} \\
z^{5} \mathrm{G}_{4}^{\circ} \\
z^{5} \mathrm{G}_{3}^{\circ} \\
z^{3} \mathrm{G}_{2}^{\circ}\end{array}$ & $\begin{array}{l}35483.89 \\
35193.13 \\
34946.55 \\
34745.72 \\
34592.72\end{array}$ & $\begin{array}{l}290.26 \\
246.58 \\
200.83 \\
153.00\end{array}$ & $\begin{array}{l}1.16 \\
1.14 \\
0.93 \\
0.31\end{array}$ & $e^{5} \mathrm{P}, e^{5} \mathrm{D}, a^{3} \mathrm{~F}, e^{5} \mathrm{~F}, e^{5} \mathrm{G}, e^{5} \mathrm{H}, a^{3} \mathrm{~F}, b^{3} \mathrm{~F}$. \\
\hline $3 d^{2} 4 s 4 p\left({ }^{4} \mathrm{~F}\right)$ & $\begin{array}{l}y^{5} \mathrm{G}_{6}^{\circ} \\
y^{5} \mathrm{G}_{5}^{\circ} \\
y^{5} \mathrm{G}_{4}^{\circ} \\
y^{5} \mathrm{G}_{3}^{\circ} \\
y^{5} \mathrm{G}_{2}^{\circ} \\
1_{4}^{\circ} \\
2_{3}^{\circ} \\
3_{3}^{\circ}\end{array}$ & $\begin{array}{l}67795.7: ? \\
67356.0: \\
66962.7: \\
66667.3: \\
66228.4: \\
62761.9 \\
76405.4 \\
79040.4\end{array}$ & $\begin{array}{l}439.7 \\
393.3 \\
295.4 \\
438.9\end{array}$ & & $\begin{array}{l}a^{5} \mathrm{~F} ?, b^{3} \mathrm{D} ?, a^{3} \mathrm{~F} ?, c^{3} \mathrm{~F} ?, d^{3} \mathrm{~F} ?, b^{3} \mathrm{G} ? . \\
a^{1} \mathrm{~F}, a^{1} \mathrm{H}, a^{5} \mathrm{P}, a^{5} \mathrm{~F} \\
a^{3} \mathrm{P}, a^{3} \mathrm{D}, b^{3} \mathrm{D}, c^{3} \mathrm{~F}, d^{3} \mathrm{~F}, b^{3} \mathrm{G}, b^{3} \mathrm{H} . \\
a^{1} \mathrm{~F}, b^{3} \mathrm{D}, c^{3} \mathrm{~F}, d^{3} \mathrm{~F} .\end{array}$ \\
\hline
\end{tabular}


TABLE 4.-Intervals of some VII terms

\begin{tabular}{|c|c|c|c|c|c|c|c|}
\hline \multirow{2}{*}{ Term } & \multirow{2}{*}{$\begin{array}{l}\text { Level in- } \\
\text { tervals }\end{array}$} & \multicolumn{2}{|c|}{ Interval ratios } & \multirow{2}{*}{ Term } & \multirow{2}{*}{$\begin{array}{l}\text { Level in- } \\
\text { tervals }\end{array}$} & \multicolumn{2}{|c|}{ Interval ratios } \\
\hline & & Observed & $\begin{array}{l}\text { Theoret- } \\
\text { ical }\end{array}$ & & & Observed & $\begin{array}{c}\text { Theoret- } \\
\text { ical }\end{array}$ \\
\hline$a^{3} \mathrm{P}$ & $\begin{array}{l}393.51 \\
219.25\end{array}$ & $\begin{array}{l}2.00 \\
1.11\end{array}$ & $\begin{array}{l}2 \\
1\end{array}$ & & 82.19 & 2.11 & 2 \\
\hline$d{ }^{3} \mathrm{P}$ & $\begin{array}{l}-258.48 \\
-120.80\end{array}$ & $\begin{array}{l}2.00 \\
0.94\end{array}$ & $\begin{array}{l}2 \\
1\end{array}$ & $z^{3} \mathrm{~F}^{\circ}$ & $\begin{array}{l}234.58 \\
193.86\end{array}$ & $\begin{array}{l}4.00 \\
3.30\end{array}$ & $\begin{array}{l}4 \\
3\end{array}$ \\
\hline$c^{3} \mathrm{D}$ & $\begin{array}{l}-60.97 \\
-41.54\end{array}$ & $\begin{array}{l}3.00 \\
2.06\end{array}$ & $\begin{array}{l}3 \\
2\end{array}$ & $2^{3} \mathrm{G}^{\circ}$ & $\begin{array}{l}209.20 \\
169.72\end{array}$ & $\begin{array}{l}5.00 \\
4.05\end{array}$ & $\begin{array}{l}5 \\
4\end{array}$ \\
\hline$a^{3} \mathrm{~F}$ & $\begin{array}{l}255.84 \\
201.76\end{array}$ & $\begin{array}{l}4.00 \\
3.15\end{array}$ & $\begin{array}{l}4 \\
3\end{array}$ & $2^{3} \mathrm{H}^{\circ}$ & $\begin{array}{l}310.71 \\
240.76\end{array}$ & $\begin{array}{l}6.00 \\
4.66\end{array}$ & $\begin{array}{l}6 \\
5\end{array}$ \\
\hline$b^{3} \mathrm{~F}$ & $\begin{array}{l}66.32 \\
51.84\end{array}$ & $\begin{array}{l}4.00 \\
3.12\end{array}$ & $\begin{array}{l}4 \\
3\end{array}$ & $2^{3}{ }^{\circ}{ }^{\circ}$ & $\begin{array}{l}242.70 \\
198.83\end{array}$ & $\begin{array}{l}7.00 \\
5.72\end{array}$ & $\begin{array}{l}7 \\
6\end{array}$ \\
\hline$b^{3} G$ & $\begin{array}{r}111.49 \\
80.54\end{array}$ & $\begin{array}{l}5.00 \\
3.61\end{array}$ & $\begin{array}{l}5 \\
4\end{array}$ & $z^{5} \mathrm{P}^{\circ}$ & $\begin{array}{l}171.95 \\
125.35\end{array}$ & $\begin{array}{l}3.00 \\
2.09\end{array}$ & $\begin{array}{l}3 \\
2\end{array}$ \\
\hline$a^{3} \mathrm{H}$ & $\begin{array}{l}84.58 \\
76.42\end{array}$ & $\begin{array}{l}6.00 \\
5.42\end{array}$ & $\begin{array}{l}6 \\
5\end{array}$ & $z^{5} \mathrm{D}^{\circ}$ & $\begin{array}{r}10.48 \\
151.60 \\
109.59 \\
58.01\end{array}$ & $\begin{array}{l}0.20 \\
3.00 \\
2.08 \\
1.15\end{array}$ & $\begin{array}{l}4 \\
3 \\
2 \\
1\end{array}$ \\
\hline$a^{5} \mathrm{P}$ & $\begin{array}{r}146.88 \\
83.02\end{array}$ & $\begin{array}{l}3.00 \\
1.70\end{array}$ & $\begin{array}{l}3 \\
2\end{array}$ & $y^{5} \mathrm{~F}^{\circ}$ & $\begin{array}{l}260.5 \\
209.7\end{array}$ & $\begin{array}{l}5.00 \\
4.04\end{array}$ & 5 \\
\hline$a^{5} \mathrm{D}$ & $\begin{array}{r}130.32 \\
120.26 \\
70.58 \\
36.05\end{array}$ & $\begin{array}{l}4.00 \\
3.14 \\
2.16 \\
1.10\end{array}$ & $\begin{array}{l}4 \\
3 \\
2 \\
1\end{array}$ & $z^{5} \mathrm{G}^{\circ}$ & $\begin{array}{l}159.7 \\
108.7 \\
290.26\end{array}$ & $\begin{array}{l}3.07 \\
2.09 \\
6.00\end{array}$ & $\begin{array}{l}3 \\
3 \\
2 \\
6\end{array}$ \\
\hline$a^{5} \mathrm{~F}$ & $\begin{array}{l}194.58 \\
159.46 \\
121.75\end{array}$ & $\begin{array}{l}5.00 \\
4.10 \\
3.12\end{array}$ & $\begin{array}{l}5 \\
4 \\
3\end{array}$ & & $\begin{array}{l}246.58 \\
200.83 \\
153.00\end{array}$ & $\begin{array}{l}5.11 \\
4.15 \\
3.16\end{array}$ & $\begin{array}{l}5 \\
4 \\
3\end{array}$ \\
\hline
\end{tabular}

Observed magnetic splitting factors ( $g$-values) for 146 levels entered in column 5 of table 3 may be compared with Landé values by referring to the tables already mentioned [21]. In calculating $g$-values from Babcock's observations of Zeeman patterns, the actual formulas used were those given by Russell [14], and double weight was assigned to results from resolved patterns, or from combinations with one $j=0$. Single or/and uncertain determinations are followed by colons.

In table 3 the $g$-values of $c^{3} \mathrm{D}$ and $t^{3} \mathrm{D}^{\circ}$ are enclosed in parentheses. This combination was identified by the $g$-values, and therefore the entered $g$ 's are not rigorously "observed" $g$ 's. There are no other observations to check these three $g$-values.

Since both the term analysis and the Zeeman-effect data are most nearly complete for the $3 d^{3} 4 s$ configuration, we have chosen these levels to illustrate the agreement of observed and theoretical $g$-values in table 5. Comparison of the observed and theoretical sums shows that the former are about 2 percent too small. The same difference was noted by Russell and Babcock [22] in their analysis of the Zeeman effect for VI lines, and was explained as a probable error in determining the magnetic field strength at the time the observations were made nearly 20 years ago. If the observed $g$-values for VI and VII levels are increased by 2 to 3 percent, the general agreement between observed and theoretical splitting factors becomes quite satisfactory. 
TABLE 5.-Observed and theoretical g-values for $3 d^{3} 4$ s levels

\begin{tabular}{|c|c|c|c|c|c|c|c|c|}
\hline Term & $J=6$ & 5 & 4 & 3 & 2 & 1 & 0 & Sum \\
\hline $\begin{array}{l}a{ }^{5} \mathrm{~F} \\
\text { Landé }\end{array}$ & & $\begin{array}{l}1.28 \\
1.40\end{array}$ & $\begin{array}{l}\text { 1. } 30: \\
\text { 1. } 35\end{array}$ & $\begin{array}{l}1.20 \\
1.25\end{array}$ & $\begin{array}{l}0.98 \\
1.00\end{array}$ & 0.00 & & $\begin{array}{l}4.76 \\
5.00\end{array}$ \\
\hline $\begin{array}{l}a \text { s } \\
\text { Landé }\end{array}$ & & & & $\begin{array}{l}1.63 \\
1.67\end{array}$ & $\begin{array}{l}1.75 \\
1.83\end{array}$ & $\begin{array}{l}2.38 \\
2.50\end{array}$ & & $\begin{array}{l}5.76 \\
6.00\end{array}$ \\
\hline $\begin{array}{l}b^{3} \mathrm{P} \\
c^{3} \mathrm{P} \\
\text { Lande }\end{array}$ & & & & & $\begin{array}{l}1.38 \\
1.38 \\
1.50\end{array}$ & $\begin{array}{l}1.41 \\
1.38 \\
1.50\end{array}$ & 0.00 & $\begin{array}{l}2.79 \\
2.76 \\
3.00\end{array}$ \\
\hline $\begin{array}{l}b^{3} \mathrm{D} \\
c^{3} \mathrm{D} \\
\text { Landé }\end{array}$ & & & & $\begin{array}{l}\text { 1. } 31 \\
\text { 1. } 27 \text { : } \\
1.33\end{array}$ & $\begin{array}{l}\text { 1. } 23 \\
1.14 \text { : } \\
1.17\end{array}$ & $\begin{array}{c}0.58 \\
.50: \\
.50\end{array}$ & & $\begin{array}{l}3.12 \\
2.91: \\
3.00\end{array}$ \\
\hline $\begin{array}{l}a^{3} \mathrm{~F} \\
d^{3} \mathrm{~F} \\
\text { Landé }\end{array}$ & & & $\begin{array}{l}1.21 \\
1.23 \\
1.25\end{array}$ & $\begin{array}{l}1.09 \\
1.06 \\
1.08\end{array}$ & $\begin{array}{r}0.65 \\
.68 \\
.67\end{array}$ & & & $\begin{array}{l}2.95 \\
2.97 \\
3.00\end{array}$ \\
\hline $\begin{array}{l}b^{3} \mathrm{G} \\
\text { Landé }\end{array}$ & & $\begin{array}{l}1.16 \\
1.20\end{array}$ & $\begin{array}{l}1.03 \\
1.05\end{array}$ & $\begin{array}{r}0.73 \\
.75\end{array}$ & & & & $\begin{array}{l}2.92 \\
3.00\end{array}$ \\
\hline $\begin{array}{l}b^{3} \mathrm{H} \\
\text { Landé }\end{array}$ & $\begin{array}{l}1.14 \\
1.17\end{array}$ & $\begin{array}{l}1.01 \\
1.03\end{array}$ & $\begin{array}{r}0.81 \\
.80\end{array}$ & & & & & $\begin{array}{l}2.96 \\
3.00\end{array}$ \\
\hline $\begin{array}{l}a^{1} \mathrm{P} \\
b^{1} \mathrm{D} \\
b^{1 \mathrm{~F}} \\
b^{1} \mathrm{G} \\
a^{1 \mathrm{H}} \\
\text { Landé }\end{array}$ & & $\begin{array}{l}1.09 \\
1.00\end{array}$ & $\begin{array}{l}0.96 \\
1.00\end{array}$ & 1.00 & 1.01 & 0.98 & & $\begin{array}{l}5.03 \\
5.00\end{array}$ \\
\hline $\begin{array}{l}\text { Sum observed } \\
\text { Sum Landê }\end{array}$ & $\begin{array}{l}1.14 \\
1.17\end{array}$ & $\begin{array}{l}4.54 \\
4.63\end{array}$ & $\begin{array}{l}\text { 6. } 54: \\
6.65\end{array}$ & $\begin{array}{l}\text { 9. } 28: \\
9.49\end{array}$ & $\begin{array}{l}\text { 10. } 20: \\
10.51\end{array}$ & $\begin{array}{l}\text { 7. } 23: \\
7.50\end{array}$ & 0.00 & $\begin{array}{l}\text { 38. } 93: \\
40.00\end{array}$ \\
\hline
\end{tabular}

The observed combinations of each term are indicated in the last column of table 3; they may serve to exhibit the combining properties of the terms in lieu of a diagram of transitions. All combinations obey the simple selection rules $\Delta L=0, \pm 1, \pm 2$, and $\Delta J=0$ (except 0 to 0$), \pm 1$, but, as usual, not all permitted combinations have been observed. Failure to find a predicted line is occasionally explained by masking (table 2), but more generally be extremely low intensity. Relative intensities appear to be law-abiding for the most part, but in triplet-system multiples the satellite lines appear to be abnormally weak when the $L$-values are large.

The total number of observed multiplets is 572, of which 81 are singlet-singlet, 205 triplet-triplet, 29 quintet-quintet, 164 singlettriplet, 70 triplet-quintet, 8 singlet-quintet, and 15 combinations with 3 miscellaneous odd levels.

\section{ELECTRON CONFIGURATIONS AND SPECTRAL TERMS}

Vanadium has atomic number 23, and the extra-nuclear structure of normal vanadium atoms is represented by $1 s^{2} 2 s^{2} 2 p^{6} 3 s^{2} 3 p^{6} 3 d^{3} 4 s^{2}$ electrons. The last five electrons account for the V I spectrum [7]; and when one is removed by ionization, the remaining four will account 
for the $\mathrm{V}$ II spectrum. It was first believed that $\left(3 d^{3} 4 s\right){ }^{5} \mathrm{~F}$ represented the normal (unexcited) state of $\mathrm{V}^{+}$ions, but further analysis proved that $\left(3 d^{4}\right)^{5} \mathrm{D}$ had this distinction [3]. Both configurations yield quintet, triplet, and singlet terms, the former 16 terms with 38 levels, and the latter 16 terms with 34 levels. These even terms combine with a multitude of odd terms resulting from configurations that include a $p$-electron, $3 d^{3} 4 p, 3 d^{2} 4 s 4 p$, thus accounting for a majority of the observed $V$ In lines.

The low (even) terms theoretically associated with the abovementioned configurations [6] are as follows:

$3 d^{3} 4 s:{ }^{5}(\mathrm{PF}),{ }^{3}(\mathrm{PF}),{ }^{3}(\mathrm{PDFGH}),{ }^{1}(\mathrm{PDFGH}),{ }^{8} \mathrm{D},{ }^{1} \mathrm{D}$. $3 d^{4}:{ }^{6 \mathrm{D}},{ }^{3}(\mathrm{PDFGH}),{ }^{1}$ (SDFGI), ${ }^{3}(\mathrm{PF}),{ }^{1}(\mathrm{SDG})$.

Terms resulting from this analysis of the $V$ II spectrum have been paired off with each and every one of these theoretical terms, except two, the highest ${ }^{1} \mathrm{D}$ of the first configuration and the highest ${ }^{1} \mathrm{~S}$ of the second. In no other spectrum are expected terms of these two configurations so completely established. Theoretical values of the two missing levels have been calculated by Ufford [23], as follows: $\left(3 d^{3} 4 s\right)^{1} \mathrm{D}=50274$ and $\left(3 d^{4}\right){ }^{1} \mathrm{~S}=61919 \mathrm{~cm}^{-1}$. Owing to the great elevation of these metastable levels, their strongest expected combinations would be in the infrared region where no V II lines have been found.

Another configuration of electrons that might be expected to yield metastable terms in the V II spectrum is

$3 \mathrm{~d}^{2} 4 \mathrm{~s}^{2}:{ }^{3}(\mathrm{PF}),{ }^{1}(\mathrm{SDG})$,

but no evidence for this has been found among our data. In this respect the V II spectrum differs most strikingly from the Ti I spectrum, which it should resemble according to the displacement law. In fact, the relative importance of the three configurations (or even terms) above-mentioned is completely reversed in the two spectra; $3 d^{2} 4 s^{2}$ includes the ground state of Ti I but is not detected in V II, whereas $3 d^{4}$ yields the ground state of $\mathrm{V}$ II but produces only very high and relatively inconspicuous terms in Ti I. However, in both spectra the strongest lines involve $3 d^{3} 4 s$, as might be expected.

All this is in accord with the general properties of spectra of elements of the first long period. The low even configurations in the first spectra are $d^{n-2} s^{2}$ and $d^{n-1} s$, and those in second spectra are $d^{n-1} s$ and $d^{n}$. As the $d$ shell nears completion or half completion, the levels of $d^{n}$ drop below those of $d^{n-1} s$, while the available data indicate a corresponding rise of $d^{n-2} s^{2}$ relative to $d^{n-1} s$. In V II $\left(3 d^{2}\right.$ $\left.4 s^{2}\right)^{3} \mathrm{~F}$ might be expected at about $34000 \mathrm{~cm}^{-1}$ above $\left(3 d^{4}\right){ }^{5} \mathrm{D}$, but almost all of the observed lines in the spectral region where its combinations should lie have been otherwise classified.

From the configurations $3 d^{3} 4 p$ and $3 d^{2} 4 s 4 p$, the following (odd) middle terms are expected to arise:

$3 d^{3} 4 p:{ }^{5}(\mathrm{SPD}),{ }^{5}(\mathrm{DFG}),{ }^{3}(\mathrm{SPD}),{ }^{3}(\mathrm{DFG}),{ }^{3}(\mathrm{SPD}),{ }^{3}(\mathrm{PDF}),{ }^{3}(\mathrm{DFG})$,

${ }^{3}(\mathrm{PDF}),{ }^{3}(\mathrm{GHI}),{ }^{1}(\mathrm{PPD}),{ }^{1}(\mathrm{PDF}),{ }^{1}(\mathrm{DFG}),{ }^{1}(\mathrm{FGH}),{ }^{1}(\mathrm{GHI})$, $3 d^{2} 4 s 4 p:{ }^{5}$ (SPD), ${ }^{5}$ (DFG), ${ }^{3}$ (SPD), ${ }^{3}(\mathrm{DFG}),{ }^{3} \mathrm{P},{ }^{1} \mathrm{P},{ }^{3}$ (SPD), , ${ }^{1}(\mathrm{SPD})$, ${ }^{3}(\mathrm{PDF}),{ }^{1}(\mathrm{PDF}),{ }^{3}(\mathrm{DFG}),{ }^{1}(\mathrm{DFG}),{ }^{3}(\mathrm{FGH}),{ }^{1}(\mathrm{FGH})$. 
V II levels have been assigned to all the terms of $3 d^{3} 4 p$, except the last five, which are theoretically very high, but only a few could be ascribed to $3 d^{2} 4 s 4 p$.

All V II terms that, with increasing total quantum numbers, form spectral series approach as limits the normal or metastable state of $\mathrm{V}^{++}$ions, described as follows:

$3 d^{3}:{ }^{4}(\mathrm{PF}),{ }^{2}(\mathrm{PDFGH}),{ }^{2} \mathrm{D}$

A partial analysis of the V III spectrum has been published by White [24], who gave values to all these terms except ${ }^{2} \mathrm{~F}$ and the second (high) ${ }^{2} \mathrm{D}$. The normal state of $\mathrm{V}^{++}$ions is represented by $\left(3 d^{3}\right)^{4} \mathrm{~F}$, and its absolute value is estimated to be $214000 \mathrm{~cm}^{-1}$.

The first high (even) terms to be expected in series with the low (even) terms of VII are as follows:

$3 d^{3} 5 s:{ }^{5}(\mathrm{PF}),{ }^{3}(\mathrm{PF}),{ }^{3}(\mathrm{PDFGH}),{ }^{1}(\mathrm{PDFGH}),{ }^{3} \mathrm{D},{ }^{1} \mathrm{D}$.

$3 d^{3} 4 d:{ }^{5}$ (PDFGH), etc.

The latter have been recognized in whole or in part, but no trace of the former could be established. This is very surprising, since the $4 p-5 s$ transitions should be prominent because $4 s-4 p$ produces the strongest lines in the V II spectrum. However, after repeated failure to find any $3 d^{3} 5 s$ levels, it became apparent that the unclassified lines in table 1 are neither grouped nor located properly to disclose such levels. An explanation is not obvious, but the fact is regretted because it foils any attempt to derive a trustworthy spectoscopic ionization potential from our data. Investigation with some other type of source, like the Paschen hollow cathode, which favors spectral series, appears to be necessary in this case.

At the present time the only available information on the absolute value of V II terms is that given by Russell [25]. From comparisons of analogous spectra, he adopted $114600 \mathrm{~cm}^{-1}$ as the probable distance between the ground states of V II and VIII. This corresponds to an ionization potential of 14.1 volts for the $\mathrm{V}^{+}$ion.

Our assignment of observed VII terms to electron configurations and convergence limits is summarized in table 6 . These assignments are based on considerations of combining properties, relative positions of terms, level separations, and in many cases on theoretical calculations made by Ufford. Unfortunately these guiding criteria are not always quantitative or decisive, and then there is a temptation to become more or less arbitrary about the interpretation.

Indeed, the mutual perturbations of neighboring terms may result in a partial "sharing of identity." The configuration which is mainly responsible for the term may, however, often be assigned with considerable probability.

To each low even term of the configuration, $(3 d)^{3} 4 s$ corresponds a proper triad of terms of $(3 d)^{3} 4 p$, which has the same limit term in VIII. The terms of this triad are usually roughly at the same level, and their combinations with the related low terms are stronger than with other low terms (except when badly perturbed). The intensities have been given the most weight in the assignments; then the levels. 


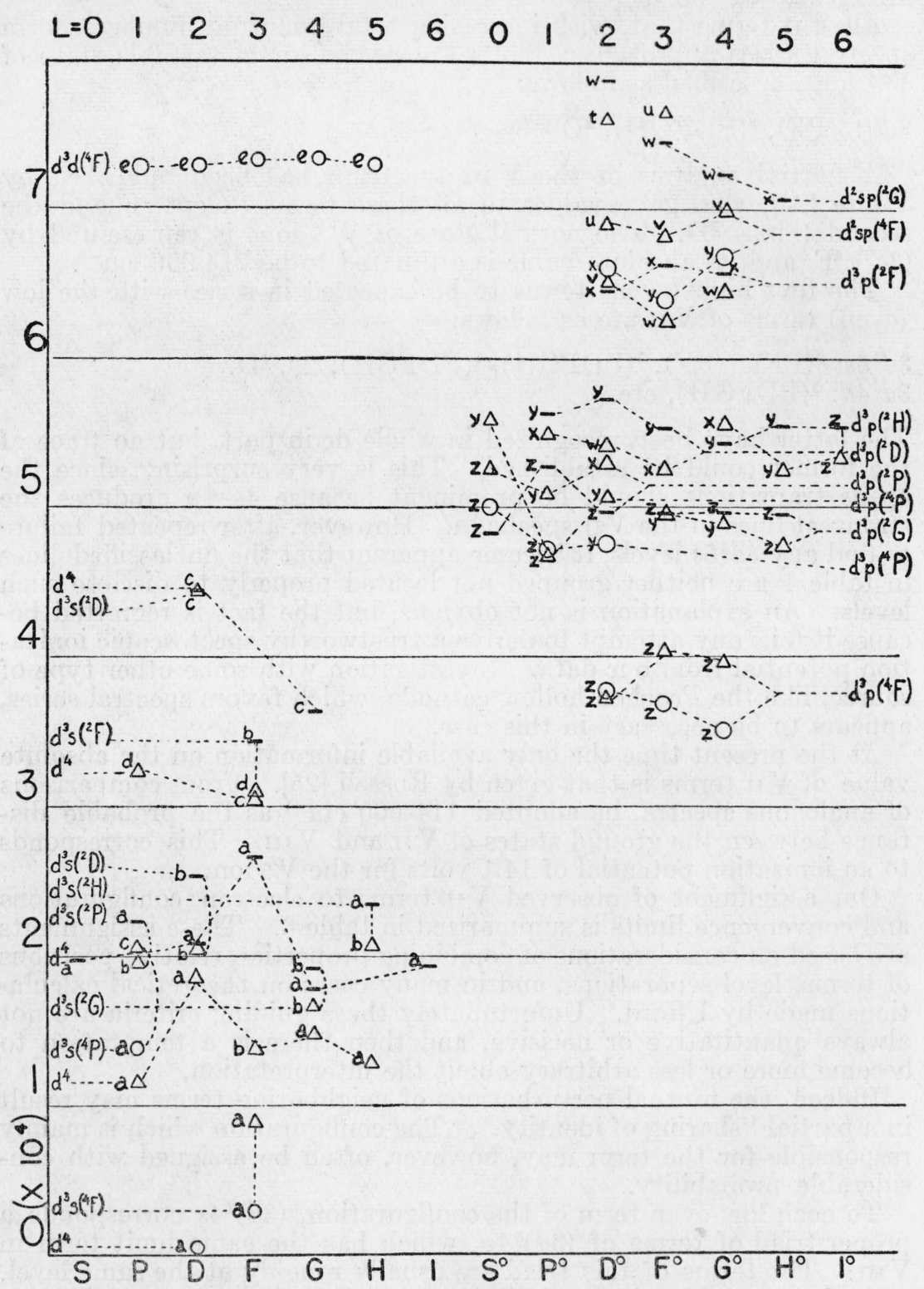

FIgURE 1.-Diagram of $\mathrm{V}$ II terms, showing relative magnitudes and assignments to configurations and limits. 
TABLE 6.-V II predicted and observed terms

\begin{tabular}{|c|c|c|c|}
\hline $\begin{array}{l}\text { Electron } \\
\text { configu- } \\
\text { ration }\end{array}$ & $\begin{array}{l}\text { Limit } \\
\text { V III }\end{array}$ & Predicted & Observed \\
\hline$(3 d)^{4}$ & & $\begin{array}{l}\text { 8D; }{ }^{3(P F)} \\
\text { 3(PDFGH) } \\
\text { 1(SDG) } \\
\text { 1(SDFG) }\end{array}$ & 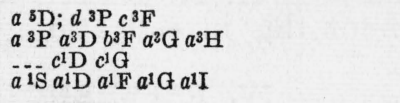 \\
\hline$(3 d)^{\prime} 48$ & $\begin{array}{l}1 \mathrm{~F} \\
2 \mathrm{P} \\
2 \mathrm{P} \\
2 \mathrm{D} \\
2 \mathrm{~F} \\
2 \mathrm{G} \\
2 \mathrm{H} \\
\left({ }^{2} \mathrm{D}\right)\end{array}$ & 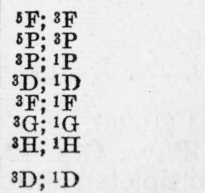 & $\begin{array}{l}a^{3} \mathrm{FF} ; a^{3} \mathrm{~F} \\
a^{3} \mathrm{P} ; c^{3} \mathrm{P} \\
b^{3} \mathrm{P} ; a^{1} \mathrm{P} \\
b^{3} \mathrm{D} ; b^{1} \mathrm{D} \\
d^{3} \mathrm{~F} ; ; b^{1} \mathrm{~F} \\
b^{3} \mathrm{G}^{1} ; b^{1} \mathrm{G} \\
b^{3} \mathrm{H} ; a^{1} \mathrm{H} \\
c^{3} \mathrm{D} ; \ldots . .\end{array}$ \\
\hline$(3 d)^{8} 4 p$ & $\begin{array}{l}{ }^{4} \mathrm{~F} \\
{ }^{4} \mathrm{P} \\
2 \mathrm{P} \\
2 \mathrm{D} \\
2 \mathrm{~F} \\
2 \mathrm{G} \\
{ }^{2} \mathrm{H} \\
\left({ }^{2} \mathrm{D}\right)\end{array}$ & 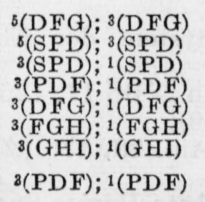 & 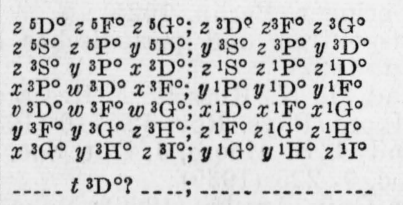 \\
\hline$(3 d)^{2} 484 p$ & $\begin{array}{l}{ }^{4} \mathrm{~F} \\
{ }_{2}^{2} \mathrm{~S} \\
2 \mathrm{P} \\
2 \mathrm{D} \\
{ }^{2} \mathrm{~F} \\
{ }^{2} \mathrm{G}\end{array}$ & 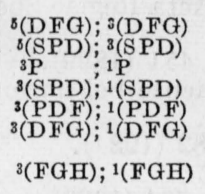 & \begin{tabular}{c}
$x{ }^{8} \mathrm{D}^{\circ} y^{8} \mathrm{~F}^{\circ} y^{8} \mathrm{G}^{\circ} ; u^{8} \mathrm{D}^{\circ} v^{8} \mathrm{~F}^{\circ} v^{8} \mathrm{G}^{\circ}$ \\
\hdashline \\
\hdashline
\end{tabular} \\
\hline$(3 d)^{3} 4 d$ & $4 F$ & ${ }^{8}$ (PDFGH) & $e^{5 \mathrm{P}} e^{5 \mathrm{D}} e^{5 \mathrm{~F}} e^{5 \mathrm{G}} e^{5 \mathrm{H}}$ \\
\hline$(3 d)^{3} 58$ & $\begin{array}{l}{ }^{4} \mathrm{~F} \\
{ }_{2}^{2} \mathrm{P} \\
{ }^{2} \mathrm{P} \\
2 \mathrm{D} \\
2 \mathrm{~F} \\
{ }^{2} \mathrm{G} \\
{ }^{2} \mathrm{H}\end{array}$ & 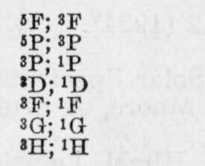 & 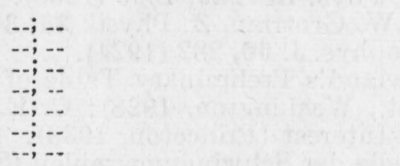 \\
\hline
\end{tabular}

The terms $d^{3} \mathrm{P}$ and $c^{3} \mathrm{~F}$ have been assigned to $(3 d)^{4}$ rather than to $(3 d)^{2}(4 s)^{2}$ because of their narrow or inverted intervals. Terms from $(3 d)^{2}(4 s)^{5}$ would have wide intervals, in normal order.

The term $x^{1} \mathrm{H}^{\circ}$ can come only from $(3 d)^{2} 4 s\left({ }^{2} \mathrm{G}\right) 4 p$; and $w^{1} \mathrm{G}^{\circ}$ and $w^{1} \mathrm{~F}^{\circ}$, being nearly at the same level, have been tentatively assigned to the same configuration.

Two very high terms $\left(w^{1} \mathrm{D}=78,791, u{ }^{3} \mathrm{~F}=76,643\right)$ have not been definitely assigned to electron configurations; they probably belong to $3 d^{2} 4 s 4 p$.

The relative positions of identified V II terms, and correlations with configurations are shown in figure 1.

There can be no doubt about the identifications and correlations of the low terms, but in the present state of our knowledge the assignment of highly excited states to definite configurations and limits cannot be guaranteed to be correct in all cases. Our greatest disappointment is our failure to establish spectral series involving transitions of a single s-type electron and thus obtain absolute values for the VII terms. 
The authors are grateful to Jerome K. Strauss for pure vanadium metal, to H. D. Babcock for permission to use and publish his Zeemaneffect data, to A. G. Shenstone for making a spectrogram in the extreme ultraviolet, to C. W. Ufford for theoretical calculations of relative terms, and especially to $\mathrm{H}$. N. Russell for advice and stimulating discussion throughout this investigation.

\section{REFERENCES}

[1] W. F. Meggers, J. Wash. Acad. Sci. 13, 317 (1923).

[2] W. F. Meggers, C. C. Kiess, and F. M. Walters, Jr., J. Opt. Soc. Am. 9, 355 (1924).

[3] W. F. Meggers, Z. Physik 33, 509 (1925); 39, 114 (1926).

[4] W. Kossel and A Sommerfeld, Verhandi. deut. Phys. Ges. 19, 241 (1919).

[5] E. Back and A. Landé, Zeemaneffekt und Multipletstruktur der Spektrallinien (Julius Springer, Berlin, 1925).

[6] F. Hund, Linienspektren und periodisches System der Elemente (Julius Springer, Berlin, 1927).

[7] W. F. Meggers and H. N. Russell, J. Research NBS 17, 126 (1936) RP906. Charlotte E. Moore, Phys. Rev. 55, 710 (1939).

[8] W. F. Meggers and C. E. Moore, J. Opt. Soc. Am. 28, 176 (A), (1938); Pub. Am. Astron. Soc. 9, 225 (1939).

[9] R. Thalén, Upsala Univ. Ärsskr. (1866); Nova Acta Regiae Soc. Sci. Upsaliensis (3) 6 (1868).

[10] N. J. Lockyer, Proc. Roy. Soc. (London) 65, 451 (1899).

[11] F. Exner and E. Haschek, Spektren der Elemente bei normalem Druck, 3, 285 (F. Deuticke, Leipzig and Vienna, 1912).

[12] A. S. King, Astrophys. J. 41, 86 (1915); 60, 282 (1924).

[13] H. D. Babcock, Astrophys. J. 34, 209 (1911).

[14] A. G. Shenstone and H. A. Blair, Phil. Mag. 8, 764 (1929). H. N. Russell, Phys. Rev. 36, 1590 (1930).

[15] H. Gieseler and W. Grotrian, Z. Physik 25, 342 (1924).

[16] A. S. King, Astrophys. J. 60, 282 (1924).

[17] Revision of Rowland's Preliminary Table of Solar Spectrum Wave lengths (Carnegie Inst., Washington, 1928); C. E. Moore, A Multiplet Table of Astrophysical Interest (Princeton, 1933).

[18] H. Kayser, Tabelle der Schwingungszahlen (S. Hirzel, Leipzig, 1925); Phys. Rev. 48, 99 (1935).

[19] H. N. Russell, A. G. Shenstone and L. Turner, Phys. Rev. 33, 900 (1929).

[20] Trans. Int. Astron. Union. 6, 100 (1938).

[21] C. C. Kiess and W. F. Meggers, BS J. Research 1, 641 (1928), RP23.

[22] H. N. Russell and H. D. Babcock, Pieter Zeeman Verhandelingen, p. 286 (1935).

[23] C. W. Ufford, Private communication.

[24] H. E. White, Phys. Rev. 33, 674 (1929).

[25] H. N. Russell, Astrophys. J. 46, 434 (1927)

Washington, February 29, 1940. 